JOURNAL OF

SYMPLECTIC GEOMETRY

Volume 7, Number 1, 29-133, 2009

\title{
GLUING PSEUDOHOLOMORPHIC CURVES ALONG BRANCHED COVERED CYLINDERS II
}

\author{
Michael Hutchings and Clifford Henry Taubes
}

This paper and its prequel ("Part I") prove a generalization of the usual gluing theorem for two index 1 pseudoholomorphic curves $U_{+}$ and $U_{-}$in the symplectization of a contact 3-manifold. We assume that for each embedded Reeb orbit $\gamma$, the total multiplicity of the negative ends of $U_{+}$at covers of $\gamma$ agrees with the total multiplicity of the positive ends of $U_{-}$at covers of $\gamma$. However, unlike in the usual gluing story, here the individual multiplicities are allowed to differ. In this situation, one can often glue $U_{+}$and $U_{-}$to an index 2 curve by inserting genus zero branched covers of $\mathbb{R}$-invariant cylinders between them. This paper shows that the signed count of such gluings equals a signed count of zeroes of a certain section of an obstruction bundle over the moduli space of branched covers of the cylinder. Part I obtained a combinatorial formula for the latter count and, assuming the result of the present paper, deduced that the differential $\partial$ in embedded contact homology satisfies $\partial^{2}=0$. The present paper completes all of the analysis that was needed in Part I. The gluing technique explained here is in principle applicable to more gluing problems. We also prove some lemmas concerning the generic behavior of pseudoholomorphic curves in symplectizations, which may be of independent interest.

\section{Contents}

1. Introduction 31

1.1. Geometric setup 31

1.2. Statement of the main result 32

1.3. Guide to the paper 34

2. Asymptotics of $J$-holomorphic curves 36

2.1. Neighborhoods of $\mathbb{R}$-invariant cylinders $\quad 37$ 
2.2. Initial decay estimates $\quad 39$

2.3. Asymptotic formula 41

3. Generic behavior of asymptotic eigenfunctions 44

3.1. Nondegenerate ends for generic $J$

3.2. Nonoverlapping pairs of ends for generic $J$

4. Genericity of immersion singularities $\quad 55$

5. The gluing construction 64

5.1. Preliminaries 65

5.2. Pregluing 66

5.3. Deforming the pregluing $\quad 67$

5.4. Equation for the deformation to be $J$-holomorphic 68

5.5. Banach space setup $\quad 72$

5.6. Solving for $\boldsymbol{\psi}_{-}$and $\boldsymbol{\psi}_{+}$in terms of $\boldsymbol{\psi}_{\boldsymbol{\Sigma}}$

5.7. Solving for $\boldsymbol{\psi}_{\boldsymbol{\Sigma}} \quad 76$

5.8. The obstruction section and the gluing map 78

6. Properties of the obstruction section $\quad 79$

6.1. Proof that $\boldsymbol{\psi}_{\boldsymbol{\Sigma}}$ is Lipschitz 79

6.2. Continuity of the obstruction section $\quad 81$

6.3. Smoothness of the obstruction section 84

6.4. Zeroes of $\mathfrak{s}$ have simple ramification points when $J$ is generic 84

$\begin{array}{ll}\text { 7. Bijectivity of the gluing map } & 87\end{array}$

7.1. Statement of the gluing theorem 87

7.2. Injectivity of the gluing map $\quad 89$

7.3. Surjectivity of the gluing map 91

8. Deforming to the linearized section $\quad 95$

8.1. The linearized section $\mathfrak{s}_{0} \quad 96$

8.2. Counting zeroes of the obstruction section and its linearization $\quad 98$

8.3. Nonlinear estimate 100

8.4. Ends with the same eigenvalue 101

8.5. Proof of the deformation result 102

$\begin{array}{ll}\text { 9. Coherent orientations } & 106\end{array}$

9.1. Algebraic preliminaries 106

9.2. A linear gluing exact sequence 107

9.3. Gluing orientations 112

9.4. Associativity of linear gluing 114

9.5. Orienting the moduli spaces 116 
10. Counting ends of the index 2 moduli space

\section{Introduction}

This paper is a sequel to [9], which we refer to here as "Part I"; references to Part I are given in the form I.*. We assume some familiarity with Sections I.1, I.2 and I.3, although we will attempt to review the essentials of what is needed here. We will not use any of Sections I.4, I.5 or I.7, and we will only rarely use Section I.6.

1.1. Geometric setup. This paper studies pseudoholomorphic curves in the symplectization of a contact 3-manifold. The setup for this is as follows: Let $Y$ be a closed oriented 3-manifold, and let $\lambda$ be a contact 1-form on $Y$. Let $\mathbf{R}$ denote the Reeb vector field associated to $\lambda$, and assume that all Reeb orbits are nondegenerate. Let $J$ be an admissible almost complex structure on $\mathbb{R} \times Y$. "Admissible" here means that $J$ is $\mathbb{R}$-invariant; $J$ sends the $\mathbb{R}$ direction, denoted by $\partial_{s}$, to $\mathbf{R}$; and $J$ sends the contact plane field $\xi=\operatorname{Ker}(\lambda)$ to itself, rotating positively with respect to $d \lambda$. If $\alpha=$ $\left(\alpha_{1}, \ldots, \alpha_{k}\right)$ and $\beta=\left(\beta_{1}, \ldots, \beta_{l}\right)$ are ordered lists of Reeb orbits, possibly repeated or multiply covered, then $\mathcal{M}^{J}(\alpha, \beta)$ denotes the moduli space of $J$ holomorphic curves $u: C \rightarrow \mathbb{R} \times Y$ with ordered and asymptotically marked positive ends at $\alpha_{1}, \ldots, \alpha_{k}$, ordered and asymptotically marked negative ends at $\beta_{1}, \ldots, \beta_{l}$, and no other ends. The precise definitions of the above notions are reviewed in Section I.1.1. We assume that the domain $C$ is a punctured compact Riemann surface, which may be disconnected, and whose components may have any genus. We also assume that $J$ is generic so that all non-multiply-covered $J$-holomorphic curves are unobstructed [5].

We want to glue together two curves $U_{+} \in \mathcal{M}^{J}\left(\alpha_{+}, \beta_{+}\right)$and $U_{-} \in$ $\mathcal{M}^{J}\left(\beta_{-}, \alpha_{-}\right)$that constitute a "gluing pair" in the sense of Definition I.1.9. This means the following: First, $U_{+}$and $U_{-}$have Fredholm index 1, cf. Section I.1.1. Second, $U_{+}$and $U_{-}$are immersed, and not multiply covered, except that they may contain unbranched covers of $\mathbb{R}$-invariant cylinders. Third, for each embedded Reeb orbit $\gamma$, the total covering multiplicity of Reeb orbits covering $\gamma$ in the list $\beta_{+}$is the same as the corresponding total for $\beta_{-}$. For example, $U_{+}$could have two negative ends at $\gamma$, while $U_{-}$could have a single positive end at the double cover of $\gamma$, which we denote by $\gamma^{2}$. 
Note that in the usual gluing story, one would assume that the lists $\beta_{+}$and $\beta_{-}$are identical; the weakening of this condition above is the novelty of the present paper. Finally, when $U_{+}$or $U_{-}$contain covers of $\mathbb{R}$-invariant cylinders over elliptic Reeb orbits, there is a fourth condition in Definition I.1.9, called "partition minimality", concerning the multiplicities of these covers. We will not review the meaning of this condition now, because it will not come to the fore in this paper until Section 8.2.

Given a gluing pair $\left(U_{+}, U_{-}\right)$as above, we want to compute a signed count of ends of the index 2 part of the moduli space $\mathcal{M}^{J}\left(\alpha_{+}, \alpha_{-}\right) / \mathbb{R}$ that are "close to breaking" into $U_{+}$and $U_{-}$together with some index zero branched covers of $\mathbb{R}$-invariant cylinders between them. This count is made precise in Definition I.1.12, reviewed here in Definition 10.1, and denoted by $\# G\left(U_{+}, U_{-}\right) \in \mathbb{Z}$. To define this count one needs to assume as above that $J$ is generic, so that all moduli spaces of non-multiply-covered $J$-holomorphic curves are smooth and have the expected dimension. To determine the signs one also needs to fix "coherent orientations" of the relevant moduli spaces; our conventions for doing so are specified in Section 9.5.

Note that the definition of $\# G\left(U_{+}, U_{-}\right)$does not count boundary points of the compactification of the moduli space $\mathcal{M}^{J}\left(\alpha_{+}, \alpha_{-}\right) / \mathbb{R}$ as in $[\mathbf{1}, \mathbf{3}]$ (for all we know there could be infinitely many such boundary points), but rather counts boundary points of a truncation of the moduli space. Consequently, this definition is insensitive to the number of levels of branched covers that may appear in the limit of a sequence of curves in $\mathcal{M}^{J}\left(\alpha_{+}, \alpha_{-}\right) / \mathbb{R}$.

1.2. Statement of the main result. Before continuing, we make two additional assumptions on our gluing pair $\left(U_{+}, U_{-}\right)$. The first is:

All negative ends of $U_{+}$, and all positive ends of $U_{-}$, are at covers of a single embedded elliptic Reeb orbit $\alpha$.

Here the statement that $\alpha$ is "elliptic" means that the linearized return map of the Reeb flow around $\alpha$ has eigenvalues on the unit circle, and thus is conjugate to a rotation by angle $2 \pi \theta$ for some $\theta \in \mathbb{R}$. Our standing assumption that all Reeb orbits are nondegenerate implies that $\theta$ is irrational.

To state the second assumption on $\left(U_{+}, U_{-}\right)$, let $a_{1}, \ldots, a_{N_{+}}$denote the multiplicities of the negative ends of $U_{+}$(this means that $U_{+}$has negative ends at the covers $\left.\alpha^{a_{1}}, \ldots, \alpha^{a_{N_{+}}}\right)$, and likewise let $a_{-1}, \ldots, a_{-N_{-}}$denote the multiplicities of the positive ends of $U_{-}$. The second assumption is:

$$
\sum_{i=1}^{N_{+}}\left\lceil a_{i} \theta\right\rceil-\sum_{i=-1}^{-N_{-}}\left\lfloor a_{i} \theta\right\rfloor=1 .
$$

To see the significance of this assumption, let

$$
\mathcal{M}:=\mathcal{M}\left(a_{1}, \ldots, a_{N_{+}} \mid a_{-1}, \ldots, a_{-N_{-}}\right)
$$


denote the moduli space of connected genus zero branched covers of $\mathbb{R} \times S^{1}$ from Definition I.2.1. Recall that a branched cover in $\mathcal{M}$ has positive ends indexed by $1, \ldots, N_{+}$, and negative ends indexed by $-1, \ldots,-N_{-}$, such that the end indexed by $i$ has multiplicity $a_{i}$; and all ends are asymptotically marked. We use a parameterization of $\alpha$ to identify elements of $\mathcal{M}$ with branched covers of the $J$-holomorphic cylinder $\mathbb{R} \times \alpha$ in $\mathbb{R} \times Y$. As explained in Section I.1.2, a branched cover of $\mathbb{R} \times \alpha$ with positive ends of multiplicities $a_{1}, \ldots, a_{N_{+}}$and negative ends of multiplicities $a_{-1}, \ldots, a_{-N_{-}}$has Fredholm index zero if and only if it consists of $\kappa_{\theta}$ genus zero components, where $\kappa_{\theta}$ denotes the left hand side of (1.2). Hence the assumption (1.2) implies that index zero branched covers of $\mathbb{R} \times \alpha$ with ends as above correspond to elements of $\mathcal{M}$.

We can now explain the idea of the gluing construction. Fix $R \gg r \gg 0$. Let $\mathcal{M}_{R}$ denote the set of branched covers in $\mathcal{M}$ such that all ramification points have $|s| \leq R$, where $s$ denotes the $\mathbb{R}$ coordinate on $\mathbb{R} \times S^{1}$. Given a branched cover $\pi: \Sigma \rightarrow \mathbb{R} \times S^{1}$ in $\mathcal{M}_{R}$, we can form a "preglued" curve by using appropriate cutoff functions to patch the negative ends of the $s \mapsto$ $s+R+r$ translate of $U_{+}$to the positive ends of $\Sigma$, and the positive ends of the $s \mapsto s-R-r$ translate of $U_{-}$to the negative ends of $\Sigma$. Now try to perturb the preglued curve to a $J$-holomorphic curve, where near the ramification points of the branched cover we only perturb in directions normal to $\mathbb{R} \times \alpha$. For a given branched cover $\Sigma$, we can obtain a (unique) $J$-holomorphic curve this way if and only if $\mathfrak{s}(\Sigma)=0$, where $\mathfrak{s}$ is a certain section of the obstruction bundle $\mathcal{O} \rightarrow \mathcal{M}_{R}$. Here the fiber of $\mathcal{O}$ over a branched cover $\Sigma$ consists of the (dual of the) cokernel of an associated linear deformation operator $D_{\Sigma}$; see Section I.2.3 for details. Note that the rank of the obstruction bundle equals the dimension of $\mathcal{M}$. In this way the count of gluings $\# G\left(U_{+}, U_{-}\right)$that we are after is related to a count of zeroes of the obstruction section $\mathfrak{s}$.

The section $\mathfrak{s}$ is difficult to understand directly, because it arises in a somewhat indirect way out of the analysis in Section 5. Fortunately, there is a more tractable section, the "linearized section" $\mathfrak{s}_{0}$, which has the same count of zeroes. The linearized section $\mathfrak{s}_{0}$ is defined explicitly in Section 8.1 in terms of the collection of "asymptotic eigenfunctions" $\gamma$ associated to the negative ends of $U_{+}$and the positive ends of $U_{-}$. (For the definition of the asymptotic eigenfunction associated to an end of a $J$-holomorphic curve, see Section 2.3.) As explained in Section I.3.2 (using results from Section 8.2), the signed count of zeroes of $\mathfrak{s}_{0}$ over $\mathcal{M}_{R}$, denoted by $\# \mathfrak{s}_{0}^{-1}(0) \in \mathbb{Z}$, is well defined as long as $\gamma$ is "admissible" in the sense of Definition I.3.2. We will prove in Section 3 that this admissibility condition holds if $J$ is generic. We also showed in Section I.3.2 that the count $\# \mathfrak{s}_{0}^{-1}(0)$ for admissible $\gamma$ does not depend on $\gamma$, but only on the multiplicities of the $\mathbb{R}$-invariant and non$\mathbb{R}$-invariant negative ends of $U_{+}$and positive ends of $U_{-}$at covers of $\alpha$. Let 
$\# \mathfrak{s}_{0}^{-1}(0)$ denote this count for admissible $\gamma$. The main result of this paper can then be stated as follows. (This appears in Part I as Theorem I.3.6.)

Theorem 1.1. Fix coherent orientations, let $J$ be a generic admissible almost complex structure on $\mathbb{R} \times Y$, and let $\left(U_{+}, U_{-}\right)$be a gluing pair satisfying assumptions (1.1) and (1.2) above. Then

$$
\# G\left(U_{+}, U_{-}\right)=\epsilon\left(U_{+}\right) \epsilon\left(U_{-}\right) \# \mathfrak{s}_{0}^{-1}(0) .
$$

Here $\epsilon\left(U_{+}\right), \epsilon\left(U_{-}\right) \in\{ \pm 1\}$ denote the signs associated to $U_{+}$and $U_{-}$by the system of coherent orientations; see Section I.1.1 and Section 9.5.

As explained in Section I.1.8, there is a straightforward generalization of this story in which the assumptions (1.1) and (1.2) are dropped. This requires gluing in disconnected branched covers. We will omit the details of this generalization, as it does not involve any new analysis and differs only in the amount of notation.

Recall from Part I that the significance of Theorem 1.1 is as follows. In Proposition I.5.1, we obtained a combinatorial formula for the count $\# \mathfrak{s}_{0}^{-1}(0)$. Combining this with Theorem 1.1 (and its generalization for disconnected branched covers) proves the main result of this pair of papers, namely Theorem I.1.13, which gives a combinatorial formula for $\# G\left(U_{+}, U_{-}\right)$. An important application of Theorem I.1.13 is given in Section I.7, which deduces that the differential $\partial$ in embedded contact homology (see $[\mathbf{8}]$ ) satisfies $\partial^{2}=0$.

Essentially the same argument shows that $\partial^{2}=0$ in the periodic Floer homology of mapping tori. In fact, our gluing theorem generalizes easily to stable Hamiltonian structures, as defined in $[\mathbf{3}, \mathbf{1 3}]$, of which contact structures and mapping tori are special cases. The starting point for the analysis in the present paper is a nice local coordinate system around a Reeb orbit, and that exists just as well in this more general setting.

1.3. Guide to the paper. This paper divides roughly into three parts.

The first part, consisting of Sections 2 to 4, does not yet address the gluing problem, but rather proves some general results on $J$-holomorphic curves in $\mathbb{R} \times Y$, which we will use in the gluing story and which might be of independent interest. In Section 2 we describe the asymptotic behavior of ends of $J$-holomorphic curves and define their asymptotic eigenfunctions. Although asymptotic results of this sort have appeared previously in $[\mathbf{7}, \mathbf{1 1}, \mathbf{1 3}]$, we will find it useful to review the asymptotics in a particular way in order to prepare for the subsequent analysis. In Section 3 we prove that for generic $J$, if $u$ is an index 1 , connected, non-multiply-covered $J$ holomorphic curve, then the asymptotic eigenfunctions describing the ends of $u$ are all nonzero; and moreover, whenever two ends of $u$ have the same "asymptotic eigenvalue", the corresponding asymptotic eigenfunctions are geometrically distinct. This is exactly what is needed to show that the collection of asymptotic eigenfunctions $\gamma$ determined by a gluing pair $\left(U_{+}, U_{-}\right)$ 
as in Section 1.2 is admissible. In Section 4 we prove that if $J$ is generic, then all non-multiply-covered $J$-holomorphic curves of index $\leq 2$ are immersed. Although this is probably not really necessary, it will simplify the analysis in the rest of the paper by allowing us to consider only immersed curves (except of course for the branched covers of cylinders that we are gluing in).

The second part of the paper, consisting of Sections 5 to 8, explains the details of gluing. In Section 5 we show how to glue $U_{+}$and $U_{-}$along a branched cover of the cylinder, wherever the obstruction section $\mathfrak{s}$ vanishes. Note that the section $\mathfrak{s}$ in Section 5 is not defined over $\mathcal{M}_{R}$ as in Section 1.2, but rather over a slightly different domain, because we are not yet modding out by the $\mathbb{R}$-action on moduli spaces of $J$-holomorphic curves. In Section 6 we prove various technical properties of the obstruction section $\mathfrak{s}$, including its continuity. In Section $\S 7$ we show that the gluing construction describes all curves that are "close to breaking" into $U_{+}$and $U_{-}$along a branched cover of the cylinder, in the precise sense of Definition I.1.10, which is reviewed in Definition 7.1. To count ends of the index 2 part of the moduli space $\mathcal{M}^{J}\left(\alpha_{+}, \alpha_{-}\right) / \mathbb{R}$, we now want to count zeroes of $\mathfrak{s}$ over a relevant slice of the quotient of its domain by the $\mathbb{R}$-action. This slice is identified with $\mathcal{M}_{R}$, minus a fringe region where $\mathfrak{s}$ has no zeroes. In Section 8 we prove that if the collection of asymptotic eigenfunctions $\gamma$ determined by $\left(U_{+}, U_{-}\right)$is admissible, then whenever $R \gg r \gg 0$, the signed count of zeroes of $\mathfrak{s}$ on $\mathcal{M}_{R}$ is the same as that of the linearized section $\mathfrak{s}_{0}$ :

$$
\# \mathfrak{s}^{-1}(0)=\# \mathfrak{s}_{0}^{-1}(0) .
$$

For the precise statement see Corollary 8.6 and Remark 8.5. In the proof of (1.4), the admissibility condition on $\gamma$ is needed to ensure that no zeroes of the section cross the boundary of $\mathcal{M}_{R}$ as we deform $\mathfrak{s}$ to $\mathfrak{s}_{0}$.

As described previously, the count $\# G\left(U_{+}, U_{-}\right)$of relevant ends of the index 2 part of the moduli space $\mathcal{M}^{J}\left(\alpha_{+}, \alpha_{-}\right) / \mathbb{R}$ can be identified with a count of zeroes of $\mathfrak{s}$ on $\mathcal{M}_{R}$. However, equation (1.4) does not yet prove Theorem 1.1, because the signs with which the zeroes of $\mathfrak{s}$ are counted in (1.4) are determined by canonical orientations of the obstruction bundle and of the moduli space of branched covers, and might not agree with the signs (coming from the coherent orientations) with which the ends of $\mathcal{M}^{J}\left(\alpha_{+}, \alpha_{-}\right) / \mathbb{R}$ are counted. This brings us to the third and last part of the paper, which is a detailed discussion of signs, occupying Sections 9 and 10. To go from equation (1.4) to Theorem 1.1, in Section 10 we will prove Theorem 10.2, asserting that if $R \gg r \gg 0$, then

$$
\# G\left(U_{+}, U_{-}\right)=\epsilon\left(U_{+}\right) \epsilon\left(U_{-}\right) \# \mathfrak{s}^{-1}(0) .
$$

To prove (1.5), it turns out that for generic $R$, each side of the equation is a signed count of points in the same finite set, so we just need to compare the 
signs. To set up this comparison, we need to rework the theory of coherent orientations from scratch, which is what we do in Section 9.

That completes the outline of the paper. Before plunging into the details, let us briefly indicate the basic idea of the gluing analysis. (This is adapted from a technique pioneered by Donaldson in the context of four-dimensional gauge theory; see [4, Ch. 7].) Suppose we want to glue together some curves $u_{1}, \ldots, u_{n}$ in some configuration. For simplicity, suppose that each $u_{i}$ is immersed with domain $C_{i}$. To start, we can use appropriate cutoff functions to form a preglued curve $C_{0}$. Now if $\psi_{i}$ is a section of the normal bundle to $C_{i}$ for each $i$, then we can deform $C_{0}$ in the direction $\beta_{1} \psi_{1}+\cdots+\beta_{n} \psi_{n}$, where $\beta_{i}$ is a cutoff function supported over the part of $C_{0}$ coming from $C_{i}$. The deformed pregluing will be pseudoholomorphic if and only if an equation of the form

$$
\beta_{1} \Theta_{1}\left(\psi_{1}, \ldots, \psi_{n}\right)+\cdots+\beta_{n} \Theta_{n}\left(\psi_{1}, \ldots, \psi_{n}\right)=0
$$

holds. Here $\Theta_{i}$ is defined on all of $C_{i}$, and has the form

$$
\Theta_{i}=D_{i} \psi_{i}+\cdots,
$$

where $D_{i}$ is the linear deformation operator associated to $C_{i}$, and the remaining terms are mostly nonlinear and involve the $\psi_{j}$ 's for those $j$ such that $C_{i}$ is adjacent to $C_{j}$ in the gluing configuration. If one sets this up properly, then the contraction mapping theorem in a suitable Banach space finds a unique $n$-tuple $\left(\psi_{1}, \ldots, \psi_{n}\right)$ such that $\psi_{i} \perp \operatorname{Ker}\left(D_{i}\right)$ and

$$
\Theta_{i}\left(\psi_{1}, \ldots, \psi_{n}\right) \in \operatorname{Coker}\left(D_{i}\right)
$$

for each $i$. If the $n$-tuple $\left(u_{1}, \ldots, u_{n}\right)$ varies over some moduli space, then these elements of Coker $\left(D_{i}\right)$ define an obstruction section over this moduli space, and we will obtain a pseudoholomorphic curve wherever this obstruction section vanishes. Further analysis shows that this construction identifies the zero set of the obstruction section with the set of all gluings in an appropriate sense. Finally, the main contribution to $\Theta_{i}$, other than $D_{i} \psi_{i}$, arises from the failure of the original preglued curve to be pseudoholomorphic, which is essentially determined by the asymptotic behavior of the $u_{i}$ 's; and this is what we use to define the "linearized section". We expect that this technique can be applied to additional gluing problems.

\section{Asymptotics of $J$-holomorphic curves}

Let $J$ be an admissible almost complex structure on $\mathbb{R} \times Y$, and let $u: C \rightarrow$ $\mathbb{R} \times Y$ be a $J$-holomorphic curve. In this section, we prove an asymptotic formula, stated in Proposition 2.4 below, for the behavior of $u$ on each end of $C$. Similar asymptotic formulas have been previously established in $[\mathbf{7}, \mathbf{1 1}, \mathbf{1 3}]$. However we will find it useful to go over the asymptotics in a particular way in order to prepare for the subsequent analysis. 
2.1. Neighborhoods of $\mathbb{R}$-invariant cylinders. We begin by writing down equations for $J$-holomorphic curves near a Reeb orbit. These equations will be used throughout the paper.

Let $\mathbf{R}$ denote the Reeb vector field on $Y$. Let $\alpha$ be an embedded Reeb orbit; by rescaling the $s$ and $t$ coordinates on $\mathbb{R} \times Y$, we may assume that $\alpha$ has period $2 \pi$. Fix a parameterization $\alpha: S^{1} \rightarrow Y$ with $\alpha^{\prime}(t)=\mathbf{R}$. Recall that the admissible almost complex structure $J$ sends the $\mathbb{R}$ direction $\partial_{s}$ to the Reeb vector field $\mathbf{R}$, so $J\left(\partial_{s}\right)=\alpha^{\prime}(t)$ on $\mathbb{R} \times \alpha$.

We begin the analysis by choosing coordinates on a tubular neighborhood of $\mathbb{R} \times \alpha$ in $\mathbb{R} \times Y$, via an "exponential map" $e$ with certain nice properties.

Lemma 2.1. For each embedded Reeb orbit $\alpha: S^{1} \rightarrow Y$, there exists a disc $D \subset \mathbb{C}$ containing the origin and an embedding

$$
e: \mathbb{R} \times S^{1} \times D \longrightarrow \mathbb{R} \times Y
$$

with the following properties:

- $e(s, t, 0)=(s, \alpha(t))$.

- The derivative of e at $(s, t, 0)$ sends $T_{0} D=\mathbb{C}$ to the contact plane $\xi_{\alpha(t)}$.

- e commutes with translations of the $\mathbb{R}$ coordinate.

- The restriction of e to each disc $\{s\} \times\{t\} \times D$ is J-holomorphic.

Proof. This can be proved similarly to [14, Lem. 5.4].

We denote the coordinates on $\mathbb{R} \times S^{1} \times D$ by $(s, t, w)$, and write $z:=s+i t$ and $w=: x+i y$.

Recall that a "positive end of $u$ at $\alpha$ " is an end of $u$ whose constant $s$ slices converge as $s \rightarrow+\infty$ to $\alpha$. By positivity of intersections with the $J$-holomorphic discs $e(\{s\} \times\{t\} \times D)$, such an end pulls back via $e$ to the graph of a smooth map

$$
\eta:\left[s_{0}, \infty\right) \times S^{1} \longrightarrow D
$$

with $\lim _{s \rightarrow \infty} \eta(s, t)=0$.

We now write down an equation for the end described by $\eta$ to be $J$ holomorphic. The conditions in Lemma 2.1 imply that in the image of $e$,

$$
T^{1,0}(\mathbb{R} \times Y)=\operatorname{span}(d z-a d \bar{z}, d w+b d \bar{z}),
$$

where $a$ and $b$ are smooth functions of $t$ and $w$ which vanish where $w=0$. It follows from (2.1) that the graph of $\eta$ is $J$-holomorphic if and only if

$$
\frac{\partial \eta}{\partial \bar{z}}+a \frac{\partial \eta}{\partial z}+b=0
$$

To see this, note that the tangent space to the graph of $\eta$ is the kernel of the $\mathbb{C}$-valued 1-form $d w-d \eta$. The latter can be written as a linear combination of the forms on the right side of (2.1), plus $d \bar{z}$ times the left side of (2.2). 
Thus (2.2) holds if and only if the tangent space to the graph of $\eta$ is $J$ invariant.

Equation (2.2) can be rewritten as

$$
\frac{\partial \eta}{\partial \bar{z}}+\nu \eta+\mu \bar{\eta}+r_{0}+r_{1} \frac{\partial \eta}{\partial z}=0
$$

where $\nu$ and $\mu$ denote the functions of $t$ given by the derivatives of $b$ at $w=0$ with respect to $w$ and $\bar{w}$, respectively, while $r_{0}$ and $r_{1}$ are smooth functions of $t$ and $w$. Since $a$ and $b$ both vanish where $w=0$, the nonlinear terms in (2.3) are bounded by

$$
\left|r_{0}(t, w)\right| \leq c|w|^{2}, \quad\left|r_{1}(t, w)\right| \leq c|w|,
$$

where $c$ denotes a constant which does not depend on $t$ or $w$.

The inequalities (2.4) lead to the following elliptic estimate for solutions to equation (2.3), which will be used frequently below. Given $z \in \mathbb{R} \times$ $S^{1}$, let $B(z, 1)$ denote the ball of radius 1 centered at $z$. In the lemma that follows, $\nabla$ is used to denote the $\mathbb{C}$-valued 1 -form of first derivatives along $\mathbb{R} \times S^{1}$. Meanwhile, $\nabla^{k}$ denotes the associated $\mathbb{C}$-valued tensor of $k$ th derivatives. Elsewhere in this paper, $\nabla$ will denote the covariant derivative on the indicated section of whatever vector bundle is under consideration, and $\nabla^{k}$ for $k \geq 1$ the associated tensor of $k$ th order covariant derivatives.

Lemma 2.2. Given functions $r_{0}$ and $r_{1}$ satisfying (2.4), there exists a positive constant $\varepsilon$, and for each nonnegative integer $k$ a constant $c_{k}$, such that the following holds. Let $\eta$ be a solution to $(2.3)$ on $\left[R_{-}-1, R_{+}+1\right] \times S^{1}$ with $|\eta| \leq \varepsilon$. (We allow $R_{ \pm}= \pm \infty$.) Then for each $z \in\left[R_{-}, R_{+}\right] \times S^{1}$, we have

$$
\left|\nabla^{k} \eta(z)\right|^{2} \leq c_{k}^{2} \int_{B(z, 1)}|\eta|^{2} .
$$

Proof. Choose $\varepsilon$ small enough that if $|\eta|<\varepsilon$, then $\left|r_{1}\right|<1 / 2$, so that the derivative term in (2.3) is elliptic. The lemma then follows by a standard bootstrapping argument, e.g., using [12, Thm. 5.5.3].

It proves useful to further rewrite equation (2.3) in terms of the asymptotic operator $L:=L_{\alpha}$ associated to the Reeb orbit $\alpha$. Our convention is to define

$$
L: C^{\infty}\left(S^{1}, \alpha^{*} \xi\right) \longrightarrow C^{\infty}\left(S^{1}, \alpha^{*} \xi\right)
$$

by

$$
L:=J \nabla_{t}^{\mathbf{R}}
$$

where $\nabla^{\mathbf{R}}$ denotes the symplectic connection on $\alpha^{*} \xi$ defined by the linearized Reeb flow. (In the literature, the operator $L$ is often defined with the opposite sign.) Recall from Section I.2.2 that since the connection $\nabla^{\mathbf{R}}$ is symplectic, the operator $L$ is self-adjoint; and since the Reeb orbit $\alpha$ is assumed nondegenerate, the spectrum of $L$ does not contain 0 . 
If we use the coordinate $w$ to trivialize the bundle $\alpha^{*} \xi$, then it follows from the admissibility condition $J \partial_{s}=\mathbf{R}$ and equation (2.1) that

$$
L \eta=i \partial_{t} \eta+2(\nu \eta+\mu \bar{\eta}) .
$$

Hence, equation (2.3) can be rewritten as

$$
\partial_{s} \eta+L \eta+\mathfrak{r}=0,
$$

where $\mathfrak{r}$ is shorthand for $2\left(r_{0}+r_{1} \partial \eta / \partial z\right)$.

The above discussion generalizes to describe a positive end of $u$ at the $m$-fold cover $\alpha^{m}$ of $\alpha$, where $m$ is a positive integer. Let $\widetilde{S}^{1}:=\mathbb{R} / 2 \pi m \mathbb{Z}$ denote the $m$-fold cover of $S^{1}$. A positive end of $u$ at $\alpha^{m}$ is then described by a function $\eta:\left[s_{0}, \infty\right) \times \widetilde{S^{1}} \rightarrow D$ satisfying the modification of equation (2.3), in which the functions $\nu, \mu, r_{0}$ and $r_{1}$ on $S^{1} \times D$ are replaced by their pullbacks to $\widetilde{S^{1}} \times D$. The latter equation can also be written as

$$
\partial_{s} \eta+L_{m} \eta+\mathfrak{r}=0
$$

where $L_{m}$ denotes the asymptotic operator associated to $\alpha^{m}$, acting on sections of the bundle $\left(\alpha^{m}\right)^{*} \xi$ over $\widetilde{S^{1}}$. Note that a solution $\eta$ to $(2.8)$ also satisfies the elliptic estimate (2.5).

2.2. Initial decay estimates. We now derive a decay estimate for solutions to equation (2.7). Let $E_{+}$and $E_{-}$, respectively, denote the smallest positive and largest negative eigenvalues of the asymptotic operator $L$. Also, let $\Pi_{+}$and $\Pi_{-}$, respectively, denote the $L^{2}\left(S^{1} ; \mathbb{R}^{2}\right)$ projections to the direct sums of the positive and negative eigenspaces of $L$.

Lemma 2.3. There exist a positive constant $\varepsilon_{0}$ and constants $c_{k}$ for each nonnegative integer $k$ with the following property. Let $\eta$ be a solution to equation (2.7) defined on $\left[R_{-}-1, R_{+}+1\right] \times S^{1}$; we allow $R_{ \pm}= \pm \infty$. Assume that $|\eta| \leq \varepsilon$, where $\varepsilon<\varepsilon_{0}$. Then for $s \in\left[R_{-}+2, R_{+}-2\right]$, we have

$$
\left|\nabla^{k} \eta\right| \leq c_{k} \varepsilon\left[e^{-E_{+}\left(s-R_{-}\right)}+e^{-E_{-}\left(s-R_{+}\right)}\right] .
$$

Proof. Below, $c$ denotes a constant which is independent of $\varepsilon, \eta$, and $R_{ \pm}$, but which may change from line to line.

To start, each solution $\eta$ to the nonlinear equation (2.7) satisfies an associated linear equation, depending on $\eta$, of the form

$$
\frac{\partial \eta}{\partial s}+L \eta+\mathfrak{r}_{0} \cdot \eta+\mathfrak{r}_{1} \cdot \nabla \eta=0 .
$$

Here $\mathfrak{r}_{0}$ and $\mathfrak{r}_{1}$ are smooth, $\mathbb{R}$-linear bundle maps with norm bounded by $c \cdot \varepsilon$. Using (2.6) to express the derivative $\partial / \partial t$ in terms of $L$, we can rewrite equation (2.10) as

$$
\frac{\partial \eta}{\partial s}+L \eta+\mathfrak{b}_{0} \cdot \eta+\mathfrak{b}_{1} \cdot L \eta=0
$$


Here $\mathfrak{b}_{0}$ and $\mathfrak{b}_{1}$ are also $\mathbb{R}$-linear bundle maps with norm bounded by some constant $c$ times $\varepsilon$.

To analyze (2.11), for $s \in\left[R_{-}, R_{+}\right]$define $f_{ \pm}(s)$ to be one half of the square of the $L^{2}$ norm of $|L|^{1 / 2} \Pi_{ \pm} \eta$ on the circle $\{s\} \times S^{1}$. Assume that $\varepsilon$ is much smaller than $\left|E_{ \pm}\right|$. Applying the projection $\Pi_{+}$to (2.11), and taking the $L^{2}\left(S^{1} ; \mathbb{R}^{2}\right)$ inner product with $L \Pi_{+} \eta$ at $s$, gives a differential inequality

$$
\frac{\partial}{\partial s} f_{+}+\left\langle L \Pi_{+} \eta, L \Pi_{+} \eta\right\rangle \leq c \varepsilon\left(\left\langle L \Pi_{+} \eta, L \Pi_{+} \eta\right\rangle+\left\langle L \Pi_{-} \eta, L \Pi_{-} \eta\right\rangle\right) .
$$

Likewise, applying $\Pi_{-}$to $(2.11)$ and taking the inner product with $L \Pi_{-} \eta$ gives

$$
-\frac{\partial}{\partial s} f_{-}+\left\langle L \Pi_{-} \eta, L \Pi_{-} \eta\right\rangle \leq c \varepsilon\left(\left\langle L \Pi_{+} \eta, L \Pi_{+} \eta\right\rangle+\left\langle L \Pi_{-} \eta, L \Pi_{-} \eta\right\rangle\right) .
$$

Adding $\varepsilon_{*}:=c \varepsilon /(1-c \varepsilon)$ times the second inequality to the first gives

$$
\frac{d}{d s}\left(f_{+}-\varepsilon_{*} f_{-}\right)+\left(1-\varepsilon_{*}\right)\left\langle L \Pi_{+} \eta, L \Pi_{+} \eta\right\rangle \leq 0 .
$$

This last inequality implies that

$$
\frac{d}{d s}\left(f_{+}-\varepsilon_{*} f_{-}\right)+2\left(1-\varepsilon_{*}\right) E_{+} f_{+} \leq 0
$$

and thus

$$
\frac{d}{d s}\left(f_{+}-\varepsilon_{*} f_{-}\right)+2\left(1-\varepsilon_{*}\right) E_{+}\left(f_{+}-\varepsilon_{*} f_{-}\right) \leq 0 .
$$

Now suppose that $R_{-}$and $R_{+}$are finite. Integrating (2.12) gives

$$
\left(f_{+}-\varepsilon_{*} f_{-}\right)(s) \leq e^{-2 \nu_{+}\left(s-R_{-}\right)}\left(f_{+}-\varepsilon_{*} f_{-}\right)\left(R_{-}\right)
$$

for $s \geq R_{-}$, where $\nu_{+}:=\left(1-\varepsilon_{*}\right) E_{+}$. A similar sequence of manipulations finds

$$
\left(f_{-}-\varepsilon_{*} f_{+}\right)(s) \leq e^{-2 \nu_{-}\left(s-R_{+}\right)}\left(f_{-}-\varepsilon_{*} f_{+}\right)\left(R_{+}\right)
$$

for $s \leq R_{+}$, where $\nu_{-}:=\left(1-\varepsilon_{*}\right) E_{-}$. The preceding two equations can be combined to obtain

$$
\begin{aligned}
& f_{+}(s) \leq\left(1-\varepsilon_{*}^{2}\right)^{-1}\left[f_{+}\left(R_{-}\right) e^{-2 \nu_{+}\left(s-R_{-}\right)}+\varepsilon_{*} f_{-}\left(R_{+}\right) e^{-2 \nu_{-}\left(s-R_{+}\right)}\right] \\
& f_{-}(s) \leq\left(1-\varepsilon_{*}^{2}\right)^{-1}\left[f_{-}\left(R_{+}\right) e^{-2 \nu_{-}\left(s-R_{+}\right)}+\varepsilon_{*} f_{+}\left(R_{-}\right) e^{-2 \nu_{+}\left(s-R_{-}\right)}\right]
\end{aligned}
$$

for $s \in\left[R_{-}, R_{+}\right]$. Since $|\eta| \leq \varepsilon$, Lemma 2.2 implies that if $\varepsilon$ is chosen sufficiently small, then $\left|f_{+}\left(R_{-}\right)\right|,\left|f_{-}\left(R_{+}\right)\right|<c \varepsilon$. Hence adding the equations (2.13) shows that if $s \in\left[R_{-}, R_{+}\right]$, then on $\{s\} \times S^{1}$,

$$
\left\||L|^{1 / 2} \eta\right\|_{2} \leq c \varepsilon\left[e^{-\nu_{+}\left(s-R_{-}\right)}+e^{-\nu_{-}\left(s-R_{+}\right)}\right] .
$$


It follows that the $L^{2}$ norm of $\eta$ over a ball of radius 1 in $\left[R_{-}, R_{+}\right] \times S^{1}$ has a bound of the same form. Lemma 2.2 then gives a pointwise bound

$$
\left|\nabla^{k} \eta\right| \leq c_{k} \varepsilon\left[e^{-\nu_{+}\left(s-R_{-}\right)}+e^{-\nu_{-}\left(s-R_{+}\right)}\right]
$$

for $s \in\left[R_{-}+1, R_{+}-1\right]$.

We now refine the estimate $(2.14)$ by feeding it back into the previous calculation. To do so, recall from (2.4) that the terms $\mathfrak{r}_{0}$ and $\mathfrak{r}_{1}$ that appear in (2.10) are bounded by $c|\eta|$. Using the bound (2.14) on the latter, we can repeat the calculations that led to (2.13), replacing the constant $\varepsilon$ by the function

$$
\widehat{\varepsilon}(s):=c_{0} \varepsilon\left[e^{-\nu_{+}\left(s-R_{-}\right)}+e^{-\nu_{-}\left(s-R_{+}\right)}\right] .
$$

If $\varepsilon$ is small, this procedure allows (2.13) to be replaced by

$$
\begin{aligned}
& f_{+}(s) \leq(1+c \varepsilon)\left[f_{+}\left(R_{-}\right) e^{-2 E_{+}\left(s-R_{-}\right)}+c \varepsilon f_{-}\left(R_{+}\right) e^{-2 E_{-}\left(s-R_{+}\right)}\right], \\
& f_{-}(s) \leq(1+c \varepsilon)\left[f_{-}\left(R_{+}\right) e^{-2 E_{-}\left(s-R_{+}\right)}+c \varepsilon f_{+}\left(R_{-}\right) e^{-2 E_{+}\left(s-R_{-}\right)}\right]
\end{aligned}
$$

for $s \in\left[R_{-}+1, R_{+}-1\right]$. The argument that gave (2.14) now gives the desired estimate $(2.9)$ for $s \in\left[R_{-}+2, R_{+}-2\right]$. Taking the limit shows that (2.9) also holds when $R_{-}=-\infty$ or $R_{+}=+\infty$.

2.3. Asymptotic formula. Fix an embedded Reeb orbit $\alpha$ and a positive integer $m$. We now prove an asymptotic formula for the behavior of a positive end of a $J$-holomorphic curve $u$ at $\alpha^{m}$. To state the result, recall that $\widetilde{S^{1}}:=\mathbb{R} / 2 \pi m \mathbb{Z}$, and let $\pi: \widetilde{S^{1}} \rightarrow S^{1}$ denote the projection. Also, let $E_{m}^{+}$and $E_{m}^{-}$, respectively, denote the smallest positive and largest negative eigenvalues of $L_{m}$.

Proposition 2.4. There exist constants $c, \kappa>0$ such that the following holds. Let $\mathcal{E}$ be a positive end of a J-holomorphic curve $u$ at $\alpha^{m}$. Then there is a real number $s_{0}$ and a function $\eta:\left[s_{0}, \infty\right) \times \widetilde{S^{1}} \rightarrow \mathbb{R}^{2}$ such that:

(a) The end $\mathcal{E}$ of $u$ is described by the map

$$
\begin{aligned}
{\left[s_{0}, \infty\right) \times \widetilde{S^{1}} } & \longrightarrow \mathbb{R} \times Y, \\
(s, t) & \longmapsto e(s, \pi(t), \eta(s, t)) .
\end{aligned}
$$

(b) There is a (possibly zero) eigenfunction $\gamma$ of $L_{m}$ with eigenvalue $E_{m}^{+}$ such that

$$
\left|\eta(s, t)-e^{-E_{m}^{+} s} \gamma(t)\right| \leq c e^{-\left(E_{m}^{+}+\kappa\right)\left(s-s_{0}\right)} .
$$

An analogous result holds for a negative end of $u$ at $\alpha^{m}$, with an analogous proof. Such an end is described by a function $\eta:\left(-\infty, s_{0}\right] \rightarrow \mathbb{R}^{2}$; and there is a (possibly zero) eigenfunction $\gamma$ of $L_{m}$ with eigenvalue $E_{m}^{-}$such that

$$
\left|\eta(s, t)-e^{-E_{m}^{-} s} \gamma(t)\right| \leq c e^{-\left(E_{m}^{-}-\kappa\right)\left(s+s_{0}\right)} .
$$


Definition 2.5. If $\mathcal{E}$ is a positive or negative end of $u$, then the asymptotic eigenfunction of the end $\mathcal{E}$ is the eigenfunction $\gamma$ of $L_{m}$ with eigenvalue $E_{m}^{+}$ or $E_{m}^{-}$appearing in $(2.16)$ or $(2.17)$, respectively. Note that the estimates (2.16) and (2.17) imply that $\gamma$ is unique.

We now prove Proposition 2.4. We already know part (a) from Section 2.1. To prove part (b), by translating the $s$ coordinate we can arrange that $|\eta| \leq \varepsilon$ on $[-2, \infty) \times \widetilde{S^{1}}$. Moreover, the analysis does not depend in any essential way on $m$, and so we may assume that $m=1$. The following lemma then implies part (b), with $s_{0}=0$.

Lemma 2.6. There exist constants $c, \kappa, \varepsilon_{0}>0$ such that the following holds. Let $\eta$ be a solution to (2.7) on $[-2, \infty) \times S^{1}$ with $|\eta| \leq \varepsilon$ where $\varepsilon<\varepsilon_{0}$. Then there is a (possibly zero) eigenfunction $\gamma$ of $L$ with eigenvalue $E_{+}$such that for $s \geq 0$,

$$
\left|\eta(s, t)-e^{-E_{+} s} \gamma(t)\right| \leq c \varepsilon e^{-\left(E_{+}+\kappa\right) s} .
$$

Proof. Suppose first that the eigenspace of $L$ with eigenvalue $E_{+}$is one dimensional. Let $\gamma_{+}$be a corresponding normalized eigenfunction. Let $\Pi_{1+}$ denote the projection to the sum of the eigenspaces with eigenvalue greater than $E_{+}$. We can then decompose $\eta$ as

$$
\eta=\eta_{-}+a_{+}(s) \gamma_{+}+\eta_{1+}
$$

where $\eta_{-}:=\Pi_{-} \eta$ and $\eta_{1+}:=\Pi_{1+} \eta$. We now individually analyze the terms on the right hand side of (2.19).

Taking the $L^{2}\left(S^{1} ; \mathbb{R}^{2}\right)$ inner product of equation $(2.7)$ with $\gamma_{+}$gives the following differential equation for $a_{+}(s)$ :

$$
\frac{d}{d s} a_{+}+E_{+} a_{+}+\left\langle\gamma_{+}, \mathfrak{r}\right\rangle=0 .
$$

This equation can be integrated to give

$$
a_{+}(s)=\widehat{a} e^{-E_{+} s}+\left.\int_{s}^{\infty} e^{-E_{+}(s-\tau)}\left\langle\gamma_{+}, \mathfrak{r}\right\rangle\right|_{\tau} d \tau,
$$

where $\widehat{a}$ is the constant

$$
\widehat{a}:=a_{+}(0)-\left.\int_{0}^{\infty} e^{E_{+} \tau}\left\langle\gamma_{+}, \mathfrak{r}\right\rangle\right|_{\tau} d \tau .
$$

In (2.20) and (2.21), the integral of $\left.e^{E_{+} \tau}\left\langle\gamma_{+}, \mathfrak{r}\right\rangle\right|_{\tau}$ on the half-line $[0, \infty)$ is absolutely convergent since $\mathfrak{r}$ is quadratic in $\eta$. Indeed, by Lemma 2.3,

$$
\int_{s}^{\infty} e^{E_{+} \tau}\left|\left\langle\gamma_{+}, \mathfrak{r}\right\rangle\right|_{\tau} \mid d \tau \leq c \varepsilon^{2} e^{-E_{+} s}
$$


Here and below, $c$ denotes a constant which does not depend on $\eta$, but which may change from one appearance to the next. Combining (2.22) with (2.20) gives

$$
\left|a_{+}(s)-\widehat{a} e^{-E_{+} s}\right| \leq c e^{-2 E_{+} s} .
$$

We now bound the size of $\eta_{-}$. Let $f_{-}(s)$ denote the $L^{2}$ norm of $\eta_{-}$on the circle $\{s\} \times S^{1}$. Taking the $L^{2}\left(S^{1} ; \mathbb{R}^{2}\right)$ inner product of equation (2.7) with $\eta_{-}$shows that

$$
\frac{d}{d s} f_{-}-\left|E_{-}\right| f_{-} \geq-\left\|\Pi_{-} \mathfrak{r}\right\|_{2},
$$

where $\|\cdot\|_{2}$ denotes the $L^{2}$ norm on the circle $\{s\} \times S^{1}$. Integrating this last equation and estimating as in (2.22) gives

$$
f_{-}(s) \leq\left. e^{\left|E_{-}\right| s} \int_{s}^{\infty} e^{-\left|E_{-}\right| \tau}\left\|\Pi_{-} \mathfrak{r}\right\|_{2}\right|_{\tau} d \tau \leq c \varepsilon^{2} e^{-2 E_{+} s} .
$$

By a standard Sobolev inequality, to obtain a pointwise bound on $\eta_{-}$, it is enough to bound the $L_{1}^{2}$ norm of $\eta_{-}$, for which purpose it suffices to bound the $L^{2}$ norm over the circle of $L \eta_{-}$. We use $g_{-}(s)$ to denote the latter function of $s$. To obtain a bound on $g_{-}(s)$, apply $L$ to equation (2.7) and take the $L^{2}$ inner product with $L \eta_{-}$to obtain

$$
\frac{d}{d s} g_{-}-\left|E_{-}\right| g_{-} \geq-\left\|\Pi_{-} L \mathfrak{r}\right\|_{2}
$$

It follows as in $(2.25)$ that $g_{-}(s) \leq c \varepsilon^{2} e^{-2 E_{+} s}$. Consequently, we also have

$$
\left|\eta_{-}(s)\right| \leq c \varepsilon^{2} e^{-2 E_{+} s}
$$

To bound $\left|\eta_{1+}\right|$, first introduce $f_{1+}(s)$ to denote the $L^{2}$ norm of $\eta_{1+}$ on $\{s\} \times S^{1}$. Steps that are analogous to those leading to $(2.24)$ find that $f_{1+}(s)$ obeys the inequality

$$
\frac{d}{d s} f_{1+}+E_{1+} f_{1+} \leq\left\|\Pi_{1+} \mathfrak{r}\right\|_{2},
$$

where $E_{1+}$ denotes the second smallest positive eigenvalue of $L$. This last equation integrates to give

$$
f_{1+}(s) \leq e^{-E_{1+} s}\left[f_{+}(0)+\left.\int_{0}^{s} e^{E_{1+} \tau}\left\|\Pi_{1+} \mathfrak{r}\right\|_{2}\right|_{\tau} d \tau\right] .
$$

Bounding the integral here using the estimate $\left\|\Pi_{1+} \mathfrak{r}\right\|_{2} \leq c \varepsilon^{2} e^{-2 E_{+} s}$, we obtain

$$
f_{1+}(s) \leq c \varepsilon\left[(1+\varepsilon) e^{-E_{1+} s}+\varepsilon e^{-2 E_{+} s}\right] .
$$

Meanwhile, the $L^{2}$ norm on $\{s\} \times S^{1}$ of $L \eta_{1+}$ obeys a differential inequality which is identical to (2.26) but for the replacement of $\mathfrak{r}$ with $L \mathfrak{r}$. Hence this $L^{2}$ norm is bounded by a constant multiple of the right hand side of (2.27). It follows that the same holds for $\left|\eta_{1+}\right|$. 
Putting together the above analysis of the terms in (2.19), we conclude that if $\kappa:=\min \left\{2 E_{+}, E_{1+}\right\}-E_{+}$, then

$$
\left|\eta-\widehat{a} e^{-E_{+} s} \gamma_{+}\right| \leq c \varepsilon e^{-\left(E_{+}+\kappa\right) s} .
$$

This proves (2.18) when the $E_{+}$eigenspace of $L$ has dimension 1 .

In the general case where the $E_{+}$eigenspace is possibly degenerate, let $\Pi_{1}$ denote the projection onto the $E_{+}$eigenspace. Then the above argument proves (2.18) with

$$
\gamma=\left.\Pi_{1} \eta\right|_{s=0}-\left.\int_{0}^{\infty} e^{E_{+} \tau} \Pi_{1} \mathfrak{r}\right|_{\tau} d \tau
$$

The integral formula (2.21) above for the asymptotic eigenfunction will play an important role in Section 3.

\section{Generic behavior of asymptotic eigenfunctions}

We have seen in Proposition 2.4 that the asymptotic behavior of an end of a $J$-holomorphic curve at a Reeb orbit $\alpha$ is controlled by an "asymptotic eigenfunction" $\gamma$ of the asymptotic operator $L_{\alpha}$. In this section we show that if the admissible almost complex structure $J$ on $\mathbb{R} \times Y$ is generic, and if $u$ is an index 1 , connected, non-multiply-covered $J$-holomorphic curve, then the asymptotic eigenfunctions associated to the ends of $u$ are all nonzero; and moreover, whenever two ends of $u$ at covers of the same Reeb orbit have the same "asymptotic eigenvalue", the corresponding asymptotic eigenfunctions are geometrically distinct. Below, the statement that "generic" admissible almost complex structures have a given property means that the space of admissible almost complex structures (with the $C^{\infty}$ Frechet space topology) contains a Baire set whose elements have the desired property.

\subsection{Nondegenerate ends for generic $J$.}

Definition 3.1. An end of a $J$-holomorphic curve at a Reeb orbit $\alpha$ is degenerate if the corresponding asymptotic eigenfunction $\gamma$ of the asymptotic operator $L_{\alpha}$ is zero.

Proposition 3.2. If the admissible almost complex structure $J$ on $\mathbb{R} \times Y$ is generic, then no index 1 , connected, non-multiply-covered J-holomorphic curve has a degenerate end.

Remark 3.3. A nice way to prove this, from [15], is to generalize the fact that moduli spaces of nonmultiply-covered $J$-holomorphic curves are smooth and have dimension equal to the index of the deformation operator for generic $J$, to consider $J$-holomorphic curves with asymptotic exponential weight constraints on the ends. If one changes the weight associated to an end so that it crosses an eigenvalue of the corresponding asymptotic 
operator, then the index of the deformation operator changes. We will use a different approach here, in order to set up the proof of Proposition 3.9 below.

Proof of Proposition 3.2. Let $\mathcal{J}$ denote the Frechet space of admissible almost complex structures. For each positive integer $n$, let $\mathcal{C}_{n}$ denote the space of pairs $(J, C)$ such that $J \in \mathcal{J}$ and $C$ is a $J$-holomorphic, connected, non-multiply covered index 1 curve with the following four properties: First, $C$ has at most $n$ ends, each of which is at a (possibly multiply covered) Reeb orbit of symplectic action at most $n$. Second, $C$ is "not close to breaking" in the sense that if $C^{\prime} \subset C$ is a connected subset with area greater than or equal to $n$ and distance less than or equal to $1 / n$ from an $\mathbb{R}$-invariant cylinder, then $C^{\prime}$ lies in an end of $C$. Third, $C$ is "not close to a multiple cover" in the sense that if $C^{\prime}$ is another $J$-holomorphic curve such that every point in $C^{\prime}$ has distance less than $1 / n$ from a point in $C$ and vice-versa, then the energy of $C^{\prime}$ is at least $2 / 3$ that of $C$. Fourth, $C$ is "not close to a nodal curve" in the sense that $C$ does not contain a simple closed curve of length less than $1 / n$ that separates $C$ into two noncompact components. Note that if $C$ is any index 1 , connected, nonmultiply covered $J$-holomorphic curve, then $(J, C) \in \mathcal{C}_{n}$ for $n$ sufficiently large. Now let $\mathfrak{p}_{n}: \mathcal{C}_{n} \rightarrow \mathcal{J}$ denote the projection. Then standard Gromov compactness arguments prove that $\mathfrak{p}_{n}^{-1}(J) / \mathbb{R}$ is compact for each $J \in \mathcal{J}$.

Next, let $\mathcal{J}_{n} \subset \mathcal{J}$ denote the set of $J \in \mathcal{J}$ such that no curve in $\mathfrak{p}_{n}^{-1}(V)$ is obstructed. By the aforementioned compactness, if $J \in \mathcal{J}_{n}$ then $\mathfrak{p}_{n}^{-1}(J) / \mathbb{R}$ is finite. A straightforward limit argument then proves that $\mathcal{J} \backslash \mathcal{J}_{n}$ is closed, so $\mathcal{J}_{n}$ is open in $\mathcal{J}$. By well-known arguments, cf. [10, Ch. 3], $\mathcal{J}_{n}$ is also dense in $\mathcal{J}$.

Now let $\widehat{\mathcal{J}}_{n} \subset \mathcal{J}_{n}$ denote the set of $J \in \mathcal{J}_{n}$ such that no curve in $\mathfrak{p}_{n}^{-1}(J)$ has a degenerate end. Since the asymptotic eigenfunctions depend continuously on $C$, it follows that $\widehat{\mathcal{J}}_{n}$ is also open in $\mathcal{J}$. Finally, we will prove:

Lemma 3.4. $\widehat{\mathcal{J}}_{n}$ is dense in $\mathcal{J}_{n}$.

Granted this lemma, we conclude that $\widehat{\mathcal{J}}_{\infty}:=\bigcap_{n} \widehat{\mathcal{J}}_{n}$ is a Baire subset of $\mathcal{J}$. This proves Proposition 3.2, because by definition, every $J \in \widehat{\mathcal{J}}_{\infty}$ obeys the condition stated in Proposition 3.2.

Proof of Lemma 3.4. Given $l \gg 0$, let $\mathcal{J}_{n}^{l}$ and $\widehat{\mathcal{J}}_{n}^{l}$ denote the analog of $\mathcal{J}_{n}$ and $\widehat{\mathcal{J}}_{n}$ defined using $C^{l}$ almost complex structures. Define $\mathfrak{p}_{n}: \mathcal{C}_{n}^{l} \rightarrow \mathcal{J}^{l}$ as above, using class $C^{l}$ pseudoholomorphic curves. It is enough to show that $\widehat{\mathcal{J}}_{n}^{l}$ is dense in $\mathcal{J}_{n}^{l}$ for all $l \gg 0$, cf. [10, $\left.\S 3\right]$.

Fix $l \gg 0$ and $J \in \mathcal{J}_{n}^{l}$. Let $\mathbf{O}$ denote the set of embedded Reeb orbits $\alpha$ for which a curve $C \in \mathfrak{p}_{n}^{-1}(J)$ has an end at a cover of $\alpha$. Note that the set $\mathbf{O}$ is finite, because $\mathfrak{p}_{n}^{-1}(J) / \mathbb{R}$ is finite. Fix a small $\delta>0$, and let $\mathcal{U} \subset \mathcal{J}_{n}^{l}$ 
be a small, contractible neighborhood of $J$ in the space of almost complex structures $J^{\prime} \in \mathcal{J}_{n}^{l}$ that agree with $J$ within distance $\delta$ of $\mathbb{R} \times \alpha$ for each $\alpha \in \mathbf{O}$.

Now fix $C \in \mathfrak{p}_{n}^{-1}(J)$, and fix a positive end $\mathcal{E}$ of $C$ at a Reeb orbit $\alpha$. In what follows, we shall assume that $\alpha$ is embedded; the argument for the general case differs only in the notation. Let $\mathcal{B}_{\mathcal{E}}$ denote the $E_{+}$eigenspace of $L$. Let $\mathcal{C}$ denote the universal moduli space consisting of pairs $\left(J^{\prime}, C^{\prime}\right)$ such that $J^{\prime} \in \mathcal{U}$ and $C^{\prime}$ is a $J^{\prime}$-holomorphic curve that is a deformation of $C$. There is an obvious projection $\pi: \mathcal{C} \rightarrow \mathcal{U}$. Since $\mathcal{U} \subset \mathcal{J}_{n}^{l}$, it follows that $\pi^{-1}\left(J^{\prime}\right)$ is one dimensional for each $J^{\prime}$, and consists of the $\mathbb{R}$-translates of a single unobstructed curve. For each pair $\left(J^{\prime}, C^{\prime}\right) \in \mathcal{C}$, the end $\mathcal{E}$ of $C$ determines an end of $C^{\prime}$ at $\alpha$, which we also denote by $\mathcal{E}$. Because $J=J^{\prime}$ along $\mathbb{R} \times \alpha$, the asymptotic operator $L:=L_{\alpha}$ is the same for $J$ and $J^{\prime}$, so the end $\mathcal{E} \subset C^{\prime}$ determines an asymptotic eigenfunction $\gamma\left(C^{\prime}\right) \in \mathcal{B}_{\mathcal{E}}$.

Fix a smooth section $\psi: \mathcal{U} \rightarrow \mathcal{C}$ with $\psi(J)=(C, J)$. We then have a function $\gamma \circ \psi: \mathcal{U} \rightarrow \mathcal{B}_{\mathcal{E}}$; let $\mathcal{Z} \subset \mathcal{U}$ denote the zero locus of $\gamma \circ \psi$. The zero locus $\mathcal{Z}$ does not depend on the choice of $\psi$, because for any $\left(J^{\prime}, C^{\prime}\right) \in \mathcal{U}$, translating $C^{\prime}$ upward by $R$ multiplies $\gamma\left(C^{\prime}\right)$ by $e^{E_{+} R}$.

Below, we will prove:

Lemma 3.5. If $\delta>0$ above is sufficiently small, then $\mathcal{Z}$ is a submanifold of $\mathcal{U}$, with $\operatorname{codim}(\mathcal{Z})=\operatorname{dim}\left(\mathcal{B}_{\mathcal{E}}\right)$.

An analogous statement also holds if $\mathcal{E}$ is a negative end of $C$, with an analogous proof.

Granted Lemma 3.5 and its negative end version, we now complete the proof of Lemma 3.4. Since there are finitely many curves in $\mathfrak{p}_{n}^{-1}(J) / \mathbb{R}$ and each has finitely many ends, if $\delta>0$ is sufficiently small then we can apply Lemma 3.5 and its negative end version a finite number of times to obtain a finite set of codimension 1 or 2 submanifolds in $\mathcal{U}$ whose complement consists of almost complex structures in $\widehat{\mathcal{J}}_{n}^{l}$. As $J$ is in $\mathcal{U}$, this proves that there are points in $\widehat{\mathcal{J}}_{n}^{l}$ that lie in any given neighborhood of $J$. Thus, $\widehat{\mathcal{J}}_{n}^{l}$ is dense in $\mathcal{J}_{n}^{l}$.

We now prepare for the proof of Lemma 3.5. It proves convenient to fix a normalized eigenfunction $\gamma_{+} \in \mathcal{B}_{\mathcal{E}}$, and write $\gamma \circ \psi=\widehat{a}_{\mathcal{E}} \gamma_{+}$, where $\widehat{a}_{\mathcal{E}}$ is a function on $\mathcal{U}$ with values in $\mathbb{R}$ or $\mathbb{C}$, depending on whether $\mathcal{B}_{\mathcal{E}}$ is one or two dimensional.

Lemma 3.6. The function $\widehat{a}_{\mathcal{E}}$ on $\mathcal{U}$ is smooth.

Proof. To simplify notation, assume that $\operatorname{dim}\left(\mathcal{B}_{\mathcal{E}}\right)=1$, so that $\widehat{a}_{\mathcal{E}}: \mathcal{U} \rightarrow \mathbb{R}$. Introduce $S:=[0, \infty) \times S^{1} \subset \mathbb{R} \times Y$, viewed as part of the cylinder $\mathbb{R} \times \alpha$. We also need weighted versions of the Sobolev spaces $L_{k=0,1,2}^{2}\left(S ; \mathbb{R}^{2}\right)$. The norm for the weighted version of $L_{k}^{2}$ assigns to a smooth, compactly supported 
function $\eta$ on $S$ the square root of

$$
\int_{S} e^{E_{+} s} \sum_{0 \leq j \leq k}\left|\nabla^{j} \eta\right|^{2} d s d t
$$

We denote the corresponding weighted Sobolev space by $L_{k+}^{2}$. Now consider equation (2.7) determined by the almost complex structure $J$ near $\alpha$.

Lemma 3.7. The space $\mathcal{M}$ of $L_{2}^{2}$ solutions to (2.7) on $S$ with small $L_{2}^{2}$ norm is a smooth manifold. Moreover, each element in $\mathcal{M}$ is in $L_{2+}^{2}$, and this inclusion defines a smooth map from $\mathcal{M}$ into $L_{2+}^{2}$. Finally, there exists a ball $B \subset \Pi_{+} L_{3 / 2}^{2}\left(S^{1} ; \mathbb{R}^{2}\right)$ about the origin, and a smooth embedding $\mathfrak{t}: B \rightarrow \mathcal{M}$ as a coordinate chart about $\eta=0$, such that $\left.\Pi_{+} \mathfrak{t}(\lambda)\right|_{s=0}=\lambda$ for all $\lambda \in B$.

Granted this lemma, we now complete the proof of Lemma 3.6. Equation (2.21) associates to each $\eta \in \mathcal{M}$ the number $\widehat{a}$, and so defines a function on $\mathcal{M}$. This function is smooth on $\mathfrak{t}(B)$. Indeed, the term $a_{+}(0)$ that appears in (2.21) is a bounded linear function on $B$. Meanwhile, the integral term in (2.21) is the pullback to $\mathcal{M}$ of a smooth function on $L_{2+}^{2}$, and so Lemma 3.7 guarantees that it too defines a smooth function on $\mathfrak{t}(B)$. With $\widehat{a}$ understood, let $J^{\prime} \in \mathcal{U}$. Since any complex structure on $\mathcal{U}$ agrees with $J$ within distance $\delta$ of $\alpha$, it follows that there exists $s_{0}>0$, and a neighborhood $\mathcal{U}^{\prime}$ of $J^{\prime}$ in $\mathcal{U}$, with the following property: If $J^{\prime \prime} \in \mathcal{U}^{\prime}$, then the end $\mathcal{E}$ of the $s \rightarrow s-s_{0}$ translate of $\psi\left(J^{\prime \prime}\right)$ restricts to $S$ as an element of the space $\mathfrak{t}(B)$. Denote this element of $\mathfrak{t}(B)$ by $\psi\left(J^{\prime \prime}\right)_{0}$. Equation (2.23) implies that

$$
\widehat{a}_{\mathcal{E}}\left(J^{\prime \prime}\right)=e^{E_{+} s_{0}} \cdot \widehat{a}\left(\psi\left(J^{\prime \prime}\right)_{0}\right) .
$$

Thus the function $\widehat{a}_{\mathcal{E}}$ on $\mathcal{U}^{\prime}$ is a constant times the pullback of $\widehat{a}$ via a smooth map from $\mathcal{U}^{\prime}$ to $\mathfrak{t}(B)$.

Proof of Lemma 3.7. Let $B_{0}$ be a small radius ball around the origin in $L_{2+}^{2}\left(S ; \mathbb{R}^{2}\right)$. Define a smooth map

$$
F: B_{0} \longrightarrow \Pi_{+} L_{3 / 2}^{2}\left(S^{1} ; \mathbb{R}^{2}\right) \times L_{1+}^{2}\left(S ; \mathbb{R}^{2}\right)
$$

by the rule

$$
\eta \longmapsto\left(\left.\Pi_{+} \eta\right|_{s=0}, \partial_{s} \eta+L \eta+\mathfrak{r}\right),
$$

where $\mathfrak{r}:=2\left(r_{0}+r_{1} \partial \eta / \partial z\right)$ as before. Note that the map $F$ is well defined if $B_{0}$ has sufficiently small radius, so that elements of $B_{0}$ have sufficiently small pointwise norm.

We claim that the differential of $F$ at the origin in $B_{0}$ is an isomorphism

$$
\left.d F\right|_{0}: L_{2+}^{2}\left(S ; \mathbb{R}^{2}\right) \stackrel{\simeq}{\longrightarrow} \Pi_{+} L_{3 / 2}^{2}\left(S^{1} ; \mathbb{R}^{2}\right) \times L_{1+}^{2}\left(S ; \mathbb{R}^{2}\right) .
$$

This claim implies the lemma by the implicit function theorem.

To prove the claim, note that

$$
\left.d F\right|_{0}(\eta)=\left(\left.\Pi_{+} \eta\right|_{s=0}, \partial_{s} \eta+L \eta\right) .
$$


To show that $\left.d F\right|_{0}$ is an isomorphism, we first need to show that given $f \in$ $\Pi_{+} L_{3 / 2}^{2}\left(S^{1} ; \mathbb{R}^{2}\right)$ and $g \in L_{1+}^{2}\left(S ; \mathbb{R}^{2}\right)$, there exists a unique $\eta \in L_{2+}^{2}\left(S ; \mathbb{R}^{2}\right)$ with $\left.\Pi_{+} \eta\right|_{s=0}=f$ and $\partial_{s} \eta+L \eta=g$. To prove the latter statement, note that any solution to these equations can be written as $\eta=\sum_{\gamma} a_{\gamma}(s) \gamma$, where the sum is over an orthonormal basis of eigenfunctions for $L$. Similarly write $g=\sum_{\gamma} g_{\gamma}(s) \gamma$ and $f=\sum_{\gamma} f_{\gamma} \gamma$. Then the $a_{\gamma}$ 's must be given as follows: If $\gamma$ is an eigenfunction with eigenvalue $E$, then

$$
a_{\gamma}(s)= \begin{cases}f_{\gamma} e^{-E s}+\int_{0}^{s} e^{-E(s-\tau)} g_{\gamma}(\tau) d \tau, & E>0 \\ -\int_{s}^{\infty} e^{|E|(s-\tau)} g_{\gamma}(\tau) d \tau, & E<0\end{cases}
$$

We must now verify that the function $\eta$ defined by (3.2) satisfies

$$
\|\eta\|_{L_{2+}^{2}} \leq c\left(\|f\|_{L_{3 / 2}^{2}}+\|g\|_{L_{1+}^{2}}\right)
$$

for some $\eta$-independent constant $c$. For this purpose, observe that the norm on $L_{k+}^{2}\left(S ; \mathbb{R}^{2}\right)$ is equivalent to the norm defined by

$$
\|\eta\|_{L_{k+}^{2}}^{2}:=\int_{S} e^{E_{+} s} \sum_{i+j=k}\left|\partial_{s}^{i} L^{j} \eta\right|^{2} d s d t .
$$

Also, the norm on $L_{3 / 2}^{2}\left(S^{1} ; \mathbb{R}^{2}\right)$ can be defined by

$$
\|f\|_{L_{3 / 2}^{2}}^{2}:=\int_{S^{1}}\left\langle f,|L|^{3} f\right\rangle d t
$$

Granted this, one need only establish (3.3) when $g=g_{\gamma}(s) \gamma$ and $f=f_{\gamma} \gamma$, where $\gamma$ is an eigenfunction of $L$, and $c$ does not depend on $\gamma$. This is straightforward using the preceding equations in the case where $E \neq E_{+}$. In the case $E=E_{+}$, it is also necessary to use the fact that

$$
\int_{0}^{\infty}\left|\partial_{s} a\right|^{2} e^{E_{+} s} d s \geq \frac{E_{+}^{2}}{4} \int_{0}^{\infty}|a|^{2} e^{E_{+} s} d s
$$

for any given function $a$ of $s$ that has limit zero as $s \rightarrow \infty$. This last inequality is proved by writing $E_{+} e^{E_{+} s}=\frac{d}{d s} e^{E_{+} s}$ in the right hand integral above, and integrating by parts.

Proof of Lemma 3.5. By Lemma 3.6 and the implicit function theorem, it is enough to assume that $\widehat{a}_{\mathcal{E}}(J)=0$, and construct $\left.j \in T \mathcal{U}\right|_{J}$ with $\nabla_{j} \widehat{a}_{\mathcal{E}} \neq 0$. We will proceed in five steps.

Before starting, by translating $s$ we can assume that the end $\mathcal{E}$ of $C$ is described by a map $\eta$ defined on $[-2, \infty) \times S^{1}$ such that for each $s_{0} \geq 0$, the $s \rightarrow s-s_{0}$ translate of $\eta$ restricts to $S$ as an element of $\mathfrak{t}(B)$. Also, we will assume that $C$ is immersed; the general case can be handled by introducing more notation, or by appealing to the results of Section 4 below. 
Step 1. The differential of the section $\psi: \mathcal{U} \rightarrow \mathcal{C}$ at $J$ defines a linear map $d \psi_{J}$, from the tangent space $\left.T \mathcal{U}\right|_{J}$, to the space of sections of the normal bundle $N_{C} \rightarrow C$. In this step, we derive a useful formula for $d \psi_{J}$.

If $C^{\prime}$ is any immersed surface in $\mathbb{R} \times Y$, then $C^{\prime}$ is $J$-holomorphic if and only if $\bar{\partial}_{J}\left(C^{\prime}\right)=0$, where $\bar{\partial}_{J}\left(C^{\prime}\right): T C^{\prime} \rightarrow N_{C^{\prime}}$ is the bundle map defined by

$$
\bar{\partial}_{J}\left(C^{\prime}\right):=\Pi_{N_{C^{\prime}}} \circ J \text {. }
$$

The linearization of $\bar{\partial}_{J}$ at our $J$-holomorphic curve $C$ defines a real linear operator, mapping sections of the normal bundle $N_{C}$ to sections of the complex line bundle

$$
\operatorname{Hom}^{0,1}\left(T C, N_{C}\right)=T^{0,1} C \otimes_{\mathbb{C}} N_{C} .
$$

Define $D_{C}$ to be $-i / 2$ times this operator. On the $s \geq 0$ part of the end $\mathcal{E}$ of $C$, the operator $D_{C}$ has the following form: If we identify the normal bundle $N_{C}$ with $\mathbb{C}$ via the coordinate $w$, and if we trivialize $T^{0,1} C$ using $d \bar{z}$, then $D_{C}$ sends a function $v \in C^{\infty}(S, \mathbb{C})$ to

$$
D_{C} v=\frac{\partial v}{\partial \bar{z}}+\nu v+\mu \bar{v}+\mathfrak{r}_{0 *} \cdot v+\mathfrak{r}_{1 *} \frac{\partial v}{\partial z} .
$$

Here $\mathfrak{r}_{0 *}$ is an $\mathbb{R}$-linear bundle map, and $\mathfrak{r}_{1 *}$ is a complex-valued function on $S$, satisfying

$$
\left|\mathfrak{r}_{0 *}\right|+\left|\mathfrak{r}_{1 *}\right| \leq c|\eta| .
$$

Since $C$ has index 1 and is a smooth point in its moduli space, the operator

$$
D_{C}: L_{1}^{2}\left(C, N_{C}\right) \longrightarrow L^{2}\left(C, T^{0,1} C \otimes_{\mathbb{C}} N_{C}\right)
$$

is surjective and has a one dimensional kernel. Let $D_{C}^{-1}$ denote the unique right inverse of (3.6) that maps to the orthogonal complement of $\operatorname{Ker}\left(D_{C}\right)$.

The differential $d \psi_{J}$ can now be described in terms of the operator $D_{C}^{-1}$ as follows. Let $j$ be a tangent vector at $J$ to $\mathcal{U}$. Let $j_{C} \in \operatorname{Hom}^{0,1}\left(T C, N_{C}\right)$ denote the $(0,1)$ bundle map given by $i / 2$ times the composition

$$
\left.T C \stackrel{j}{\longrightarrow} T(\mathbb{R} \times Y)\right|_{C} \stackrel{\Pi_{N_{C}}}{\longrightarrow} N_{C} .
$$

Let $\left\{J_{\tau}\right\}$ be a smooth family of almost complex structures parameterized by a neighborhood of 0 in $\mathbb{R}$ with $J_{0}=J$ and $\left.\frac{d}{d \tau}\right|_{\tau=0} J_{\tau}=j$. Write $\psi\left(J_{\tau}\right)=$ $\left(J_{\tau}, C_{\tau}\right)$. Then differentiating the equation $\bar{\partial}_{J_{\tau}}\left(C_{\tau}\right)=0$ at $\tau=0$ shows that

$$
(-2 i) j_{C}+(2 i) D_{C}\left(d \psi_{J}(j)\right)=0 .
$$

Thus, the section $d \psi_{J}(j)$ of $C$ 's normal bundle is given by

$$
d \psi_{J}(j)=D_{C}^{-1}\left(j_{C}\right)+w_{j},
$$

where $w_{j} \in \operatorname{Ker}\left(D_{C}\right)$ depends linearly on $j$.

Step 2. We now choose a tangent vector $\left.j \in T \mathcal{J}\right|_{J}$. Our $j$ will vanish identically on some neighborhood of $\mathbb{R} \times \alpha^{\prime}$ for all Reeb orbits $\alpha^{\prime}$ in $\mathbf{O}$, so that $\left.j \in T \mathcal{U}\right|_{J}$ if $\delta$ is chosen sufficiently small. (See the beginning of the 
proof of Lemma 3.4.) To finish the proof of Lemma 3.5, we will later show that $\nabla_{j} \widehat{a}_{\mathcal{E}} \neq 0$.

To prepare for the choice of $j$, we need to consider the locus where the projection of $C$ to $Y$ is not an embedding. First, let $\Lambda \subset C$ denote the set of points where $C$ intersects the $s \mapsto s-s_{0}$ translate of $C$ for some $s_{0} \neq 0$ (including $s_{0}= \pm \infty$, i.e., points where the projection of $C$ to $Y$ intersects one of the Reeb orbits at the ends of $C$ ). For any given $s_{0}$, the set of such intersections is discrete (in fact finite, see $[\mathbf{1 3}]$ ). Thus $\Lambda$ is a closed codimension 1 subvariety of $C$. Next, let $\mathcal{T} \subset C$ denote the set of points where $C$ is tangent to $\xi$ or to $\operatorname{span}\left(\partial_{s}, \mathbf{R}\right)$. The set $\mathcal{T}$ is also discrete (in fact finite).

Next, recall that an admissible almost complex structure $J$ is required to be $\mathbb{R}$-invariant and to send $\partial_{s} \mapsto \mathbf{R}$ and $\xi \rightarrow \xi$. Thus a tangent vector $j \in$ $\left.T \mathcal{J}\right|_{J}$ is equivalent to a $(0,1)$ bundle map $\xi \rightarrow \xi$ over $Y$. As a consequence, any $(0,1)$ bundle map $f: T C \rightarrow N_{C}$ can be realized as $j_{C}$ for some $\left.j \in T \mathcal{J}\right|_{J}$, provided that $f=0$ in a neighborhood of $\Lambda \cup \mathcal{T}$.

We now specify such a bundle map $f$. First of all, $f$ will be supported in the $s>1$ part of the end $\mathcal{E}$ of $C$. On this end, under the identifications in $(3.5)$, a $(0,1)$ bundle map $f: T C \rightarrow N_{C}$ is equivalent to a complex function $g$ on $[-2, \infty) \times S^{1}$. To specify $g$, fix $r>1$ large and $\rho>0$ small. Recall that $\gamma_{+}$denotes the chosen normalized eigenfunction of $L$ with eigenvalue $E_{+}$. Let $\chi:[-2, \infty) \times S^{1} \rightarrow[0,1]$ be a function which vanishes where $s \notin[r, r+1]$ or the distance to $\Lambda \cup \mathcal{T}$ is less than $\rho / 2$, and which is 1 where $s \in[r+\rho, r+1-\rho]$ and the distance to $\Lambda \cup \mathcal{T}$ is greater than $\rho$. Now define

$$
g(s, t):=\chi(s, t) \gamma_{+}(t) .
$$

This completes the specification of $f$. Finally, choose $j$ such that $f=j_{C}$.

Step 3. We now calculate $\nabla_{j} \widehat{a}_{\mathcal{E}}$. By equation (3.8),

$$
\nabla_{j} \widehat{a}_{\mathcal{E}}=\left(d \widehat{a}_{\mathcal{E}}\right)_{J} D_{C}^{-1}\left(j_{C}\right) .
$$

To see why the $w_{j}$ term in (3.8) is irrelevant, observe that the latter is in $\operatorname{Ker}\left(D_{C}\right)$, and hence it is a multiple of the tangent vector at $C$ to the 1parameter family of pseudoholomorphic curves given by translating $C$ along the $\mathbb{R}$ factor of $\mathbb{R} \times Y$. Since we are assuming that $\widehat{a}_{\mathcal{E}}(C)=0$, these translations have no effect on $\widehat{a}_{\mathcal{E}}$.

To evaluate the right hand side of (3.9), note that the restriction of $D_{C}^{-1}\left(j_{C}\right)$ to the end $\mathcal{E}$ appears as a function $v:[-2, \infty) \times S^{1} \rightarrow \mathbb{C}$. Now fix $s_{0} \geq r+1$, and let $\eta_{0}$ and $v_{0}$ denote the restrictions to $S$ of the $s \rightarrow s-s_{0}$ translates of $\eta$ and $v$, respectively. Since $j$ is supported where $r \leq s \leq r+1$, it follows using equation (3.8) that $\left.v_{0} \in T \mathcal{M}\right|_{\eta_{0}}$. So by (3.9) and (3.1),

$$
\nabla_{j} \widehat{a}_{\mathcal{E}}=\left.e^{E_{+} s_{0}}(d \widehat{a})\right|_{\eta_{0}}\left(v_{0}\right) .
$$


We conclude from this and equation (2.21) that for any $s_{0} \geq r+1$,

$$
\begin{aligned}
\nabla_{j} \widehat{a}_{\mathcal{E}}= & e^{E_{+} s_{0}} v_{+}\left(s_{0}\right)-\int_{s_{0}}^{\infty} e^{E_{+} s}\left\langle\gamma_{+}, \mathfrak{r}_{0} \cdot v+\mathfrak{r}_{1} \cdot \nabla v+d \mathfrak{r}_{0}(v) \cdot \eta\right. \\
& \left.+d \mathfrak{r}_{1}(v) \cdot \nabla \eta\right\rangle\left.\right|_{s} d s .
\end{aligned}
$$

Here $v_{+}\left(s_{0}\right)$ denotes the $L^{2}$ inner product on $S^{1}$ between $\gamma_{+}$and $v\left(s_{0}, \cdot\right)$, while $\mathfrak{r}_{0}$ and $\mathfrak{r}_{1}$ are the $w$-dependent bundle maps from (2.10).

To prove that the differential (3.10) is nonzero, we will show that for suitable $r$ and $s_{0}$, the $v_{+}$term in (3.10) is much larger than the integral.

Step 4. We now establish an upper bound on the integral in (3.10).

By definition, $v$ obeys the equation

$$
\frac{1}{2}\left(\frac{\partial v}{\partial s}+L v\right)+\mathfrak{r}_{0 *} \cdot v+\mathfrak{r}_{1 *} \cdot \frac{\partial v}{\partial z}=g
$$

Since the operator $D_{C}$ in (3.6) is bounded and Fredholm, there is a constant $c$ such that $\|\lambda\|_{2}^{2}+\|\nabla \lambda\|_{2}^{2} \leq c^{2}\left\|D_{C} \lambda\right\|_{2}^{2}$ for all $\lambda$ that are in the domain of $D_{C}$ and orthogonal to the kernel of $D_{C}$. Applying this to $\lambda:=D_{C}^{-1}\left(j_{C}\right)$, we deduce that

$$
\|v\|_{2}^{2}+\|\nabla v\|_{2}^{2} \leq c^{2}\|g\|_{2}^{2}
$$

Now observe that (3.11) is a homogeneous equation for $v$ where $s \geq$ $r+1$. Elliptic estimates as in Lemma 2.2 then give pointwise bounds for the derivatives of $v$ on the $s=r+2$ circle in terms of the $L^{2}$ norm of $v$, which by (3.12) is bounded by $c\|g\|_{2}$. In particular, the $L^{2}$ norm of $|L|^{1 / 2} v$ over the $s=r+2$ circle is bounded by an $r$ - and $\rho$-independent constant times the $L^{2}$ norm of $g$. The analysis leading to (2.9) then gives

$$
\left|\nabla^{k} v\right| \leq c_{k} e^{-E_{+}(s-r)}\|g\|_{2}
$$

where $s \geq r+3$.

Recall from Lemma 2.3 that $|\eta|$ and $|\nabla \eta|$ are bounded by $c \varepsilon e^{-E_{+} s}$ for $s \geq 0$. Combining this with (3.13), we conclude that if $s_{0} \geq r+3$, then the integral in (3.10) is bounded by

$$
\left|\int_{s_{0}}^{\infty}(\cdots) d s\right| \leq c \varepsilon e^{-E_{+}\left(s_{0}-r\right)}\|g\|_{2} .
$$

Step 5. We now establish a lower bound on the term $e^{E_{+} s_{0}} v_{+}\left(s_{0}\right)$ in (3.10).

Suppose that $r$ is large. The analysis leading to (2.9) on the cylinder where $0 \leq s \leq r$ can be employed to prove that when $s=r / 2$,

$$
\left|\nabla^{k} v\right| \leq c_{k} e^{-E_{+} r / 2}\|g\|_{2} .
$$


Therefore,

$$
\left|v_{+}(r / 2)\right| \leq c e^{-E_{+} r / 2}\|g\|_{2} .
$$

Keeping this in mind, define $g_{+}(s):=\left.\left\langle\gamma_{+}, g\right\rangle\right|_{s}$. Take the $L^{2}\left(S^{1} ; \mathbb{R}^{2}\right)$ inner product of (3.11) with $\gamma_{+}$to obtain the differential equation

$$
\frac{1}{2}\left(\frac{d v_{+}}{d s}+E_{+} v_{+}\right)+\left.\left\langle\gamma_{+}, \mathfrak{r}_{0 *} \cdot v+\mathfrak{r}_{1 *} \cdot \partial v\right\rangle\right|_{s}=g_{+} .
$$

Integrating this from $s=r / 2$ to $s=s_{0}$ gives

$$
\begin{aligned}
e^{E_{+} s_{0}} v_{+}\left(s_{0}\right)= & e^{E_{+} r / 2} v_{+}(r / 2) \\
& +2 \int_{r / 2}^{s_{0}} e^{E_{+} s}\left[g_{+}-\left.\left\langle\gamma_{+}, \mathfrak{r}_{0 *} \cdot v+\mathfrak{r}_{1 *} \cdot \partial v\right\rangle\right|_{s}\right] d s .
\end{aligned}
$$

Using (3.16), the bounds on $\eta$ and its derivatives by $c \varepsilon e^{-E_{+} s}$, and the bounds on $v$ and its derivatives in (3.13), we deduce that

$$
e^{E_{+} s_{0}} v_{+}\left(s_{0}\right) \geq 2 \int_{r / 2}^{s_{0}} e^{E_{+} s} g_{+}(s) d s-c\left(s_{0}-r / 2+1\right)\|g\|_{2} .
$$

By the definition of $g$, we have

$$
\begin{aligned}
\lim _{\rho \rightarrow 0} \int_{r / 2}^{s_{0}} e^{E_{+} s} g_{+}(s) d s & =\int_{r}^{r+1} e^{E_{+} s} d s, \\
\lim _{\rho \rightarrow 0}\|g\|_{2} & =2 \pi .
\end{aligned}
$$

Combining (3.10), (3.14), (3.17) and (3.18), we conclude that for any $r$, we can choose $\rho$ sufficiently small that for any $s_{0}$,

$$
\nabla_{j} \widehat{a}_{\mathcal{E}} \geq c_{1} e^{E_{+} r}-c_{2}\left(s_{0}-r / 2+1\right)-c_{3} e^{-E_{+}\left(s_{0}-r\right)} .
$$

If $r$ is sufficiently large and $s_{0}=2 r$, then the first term on the right hand side of (3.19) is much larger than the other two terms, so $\nabla_{j} \widehat{a}_{\mathcal{E}} \neq 0$. This completes the proof of Lemma 3.5, and with it Proposition 3.2.

3.2. Nonoverlapping pairs of ends for generic $J$. We now prove a genericity result for the asymptotic eigenfunctions associated to pairs of ends of a $J$-holomorphic curve. To state the result, recall that if $\alpha$ is an embedded Reeb orbit, if $m_{1}$ and $m_{2}$ are positive integers and if the smallest positive eigenvalues of $L_{\alpha^{m_{1}}}$ and $L_{\alpha^{m_{2}}}$ agree, then the corresponding eigenspaces are pulled back from the smallest positive eigenspace of $L_{\alpha^{m}}$, where $m$ denotes the greatest common divisor of $m_{1}$ and $m_{2}$. This is explained in Section I.3.1 for elliptic Reeb orbits, and is even easier for hyperbolic Reeb orbits. Note also that $\mathbb{Z} / m$ acts on the eigenspace of $L_{\alpha^{m}}$ with smallest positive eigenvalue, via pullback from its action on $\alpha^{m}$ by deck transformations for the covering map to $\alpha$. 
Definition 3.8. Let $C$ be a $J$-holomorphic curve with all ends nondegenerate. A pair of positive ends $\mathcal{E}_{1}, \mathcal{E}_{2}$ of $C$ is overlapping if the following conditions hold:

- There is a single embedded Reeb orbit $\alpha$, and positive integers $m_{1}$ and $m_{2}$, such that $\mathcal{E}_{i}$ is a positive end at $\alpha^{m_{i}}$.

- The smallest positive eigenvalues of $L_{\alpha^{m}}$ and $L_{\alpha^{m}}$ agree.

- Let $m$ denote the greatest common divisor of $m_{1}$ and $m_{2}$, and let $\gamma_{i}$ denote the eigenfunction of $L_{\alpha^{m}}$ whose pullback to $\alpha^{m_{i}}$ is the asymptotic eigenfunction associated to the end $\mathcal{E}_{i}$. Then there exists $g \in \mathbb{Z} / m$ such that $g \cdot \gamma_{1}=\gamma_{2}$.

An overlapping pair of negative ends is defined analogously.

Proposition 3.9. If the admissible almost complex structure $J$ on $\mathbb{R} \times Y$ is generic, then no index 1 , connected, non-multiply-covered $J$-holomorphic curve has an overlapping pair of ends.

Proof. Fix $l \gg 0$, and reintroduce the notation $\mathfrak{p}_{n}$ and $\widehat{\mathcal{J}}_{n}^{l}$ from the beginning of the proof of Proposition 3.2. Let $\widetilde{\mathcal{J}}_{n}^{l} \subset \widehat{\mathcal{J}}_{n}^{l}$ denote the set of $J \in \widehat{\mathcal{J}}_{n}^{l}$ such that no curve $C \in \mathfrak{p}_{n}^{-1}(J)$ has an overlapping pair of ends. Then as in the proof of Proposition 3.2, it is enough to show that $\widetilde{\mathcal{J}}_{n}$ is dense in $\widehat{\mathcal{J}}_{n}$.

To prove that $\widetilde{\mathcal{J}}_{n}$ is dense in $\widehat{\mathcal{J}}_{n}$, fix $J \in \widehat{\mathcal{J}}_{n}$, and let $\mathbf{O}, \delta$ and $\mathcal{U}$ be defined as in the beginning of the proof of Lemma 3.4.

Next, fix $C \in \mathfrak{p}_{n}^{-1}(J)$ with ordered positive ends at (possibly multiply covered or repeated) Reeb orbits $\alpha_{1}, \ldots, \alpha_{N_{+}}$, and ordered negative ends at Reeb orbits $\alpha_{-1}, \ldots, \alpha_{-N_{-}}$. For $i=1, \ldots, N_{+}$, let $E_{i}$ denote the smallest positive eigenvalue of the asymptotic operator $L_{\alpha_{i}}$; and for $i=-1, \ldots,-N_{-}$, let $E_{i}$ denote the largest negative eigenvalue of $L_{\alpha_{i}}$. Let $\mathcal{B}_{i}$ denote the $E_{i}$ eigenspace of $L_{\alpha_{i}}$; then the ends of $C$ determine asymptotic eigenfunctions $\gamma_{i} \in \mathcal{B}_{i} \backslash\{0\}$.

Applying the translation $s \mapsto s+R$ to $C$ acts on $\gamma_{i}$ as

$$
\gamma_{i} \longmapsto e^{E_{i} R} \gamma_{i}
$$

To keep track of this, let $I$ denote the index set $\left\{1, \ldots, N_{+}\right\} \cup\left\{-1, \ldots,-N_{-}\right\}$, and let $\mathcal{B}:=\bigoplus_{i \in I} \mathcal{B}_{i}$. Define $\mathbb{P}$ to be the set of tuples $\left(\gamma_{i}\right)_{i \in I} \in \mathcal{B}$ with all components $\gamma_{i}$ nonzero, modulo the equivalence relation

$$
\left(\gamma_{i}\right)_{i \in I} \sim\left(e^{E_{i} R} \gamma_{i}\right)_{i \in I}
$$

for all $R \in \mathbb{R}$. Note that $\mathbb{P}$ is a smooth manifold. The asymptotic eigenfunctions of the ends of $C$ define an element $p(C) \in \mathbb{P}$ which is invariant under translation of $C$. Furthermore, $C$ has all pairs of ends nonoverlapping if and only if $p(C) \in \mathbb{P} \backslash Z$, where $Z$ is a finite union of codimension 1 and 2-submanifolds of $\mathbb{P}$. 
As in the proof of Lemma 3.4, let $\pi: \mathcal{C} \rightarrow \mathcal{U}$ denote the universal moduli space, and let $\psi: \mathcal{U} \rightarrow \mathcal{C}$ be a smooth section with $\psi(J)=C$. The asymptotic eigenfunctions determine a smooth map $p: \mathcal{U} \rightarrow \mathbb{P}$, which does not depend on $\psi$.

We will momentarily prove:

Lemma 3.10. If $\delta$ is sufficiently small, then the map $p: \mathcal{U} \rightarrow \mathbb{P}$ is a submersion.

It follows from this lemma and the implicit function theorem that $p^{-1}(Z)$ is a finite union of codimension 1 and 2 submanifolds of $\mathcal{U}$. This completes the proof of Proposition 3.9 (in the same way that Lemma 3.5 completes the proof of Proposition 3.2).

Proof of Lemma 3.10. We will assume that $C$ is immersed, and also that each eigenspace $\mathcal{B}_{i}$ is one dimensional. The argument for the general case differs only in the amount of notation.

Let $\left.j_{k} \in T \mathcal{U}\right|_{J}$ denote the tangent vector that is constructed in the proof of Lemma 3.5 for the $k$ th end. Here we assume that the constant $r$ used is very large and the constant $\rho$ used is very small, and that these constants are the same for each $k$. (The construction given was for positive ends; there is a negative end construction that is completely analogous.) To prove the lemma, we will show that the set of tangent vectors $\left\{d p_{J}\left(j_{k}\right)\right\}_{k \in I}$ spans $\left.T \mathbb{P}\right|_{p(C)}$.

On the $i$ th end we introduce, as in the proof of Proposition 3.2, a normalized eigenfunction for $\mathcal{B}_{i}$, in order to view the assignment of $\gamma_{i}$ to the elements in $\mathcal{U}$ as defining a function $\widehat{a}_{\mathcal{E}_{i}}: \mathcal{U} \rightarrow \mathbb{R}$. For each pair $(i, k) \in I \times I$, we now have the following analog of equation (3.9):

$$
\nabla_{j_{k}} \widehat{a}_{\mathcal{E}_{i}}=\left.\left(d \widehat{a}_{\mathcal{E}_{i}}\right)\right|_{J}\left(D_{C}^{-1}\left(j_{k C}\right)+w_{j_{k}}\right) .
$$

Here $D_{C}^{-1}\left(j_{k C}\right)$ and $w_{j_{k}}$ are the $j_{k}$ versions of the expression on the right hand side of (3.8).

We now introduce

$$
\widehat{a}_{i k}:=\left.\left(d \widehat{a}_{\mathcal{E}_{i}}\right)\right|_{J}\left(D_{C}^{-1}\left(j_{k C}\right)\right),
$$

and we claim that $\left.(d p)\right|_{J}\left(j_{k}\right)$ depends only on $\widehat{a}_{i k}$. Indeed, this follows from the fact that each $w_{j_{k}}$ is tangent to the orbit through $C$ of the $\mathbb{R}$-action that translates curves along the $\mathbb{R}$ factor in $\mathbb{R} \times Y$. Since $p$ is invariant under this action, the $w_{j_{k}}$ term in (3.22) contributes nothing to $\left.d p\right|_{J}\left(j_{k}\right)$. Therefore, $\left.d p\right|_{J}\left(j_{k}\right)$ is the projection to $\left.T \mathbb{P}\right|_{p(C)}$ of the vector $\left(\widehat{a}_{i k}\right)_{i \in I} \in \mathcal{B}$.

So, to prove that the set of tangent vectors $\left\{d p_{J}\left(j_{k}\right)\right\}_{k \in I}$ spans $\left.T \mathbb{P}\right|_{p(C)}$, it is enough to show that the matrix $\left(\widehat{a}_{i k}\right)_{i, k \in I}$ has nonzero determinant. For this purpose, it is enough to show that the diagonal entries are much larger than the other entries. Let $i$ and $k$ be distinct ends, and to simplify notation 
assume that they are positive. By equation (3.19), if $r$ is sufficiently large and $\rho$ is sufficiently small, then

$$
\widehat{a}_{k k} \geq c e^{E_{k} r} .
$$

Next let $g_{k}$ denote the function $g:[-2, \infty) \times S^{1} \rightarrow \mathbb{C}$ used to construct $j_{k}$. We will show that

$$
\left|\widehat{a}_{i k}\right| \leq c e^{-E_{k} r / 2}\left\|g_{k}\right\|_{2} .
$$

By (3.18), this is much smaller than (3.23) when $r$ is large and $\rho$ is small.

To prove (3.24), let $v_{k}:=D_{C}^{-1}\left(j_{k}\right)_{C}$. Let $\beta: C \rightarrow[0,1]$ be a smooth function which equals 1 off of the $s \geq r / 2$ part of the $k$ th end, and which on the $k$ th end is a function of $s$ such that $\left|\beta^{\prime}\right| \leq 2$, and $\beta=0$ for $s \geq r / 2+1$. Define $v_{k}^{\prime}:=\beta v_{k}$. By definition, $D_{C} v_{k}^{\prime}$ is nonzero only on the part of the $k$ th end where $r / 2<s<r / 2+1$. Using the bound (3.15), it follows that $\left\|D_{C} v_{k}^{\prime}\right\|_{2}$ is bounded by the right side of (3.24). In particular, the $L^{2}$ norm of $v_{k}$, over the complement of the $s \geq r / 2$ part of the $k$ th end, is bounded by the right side of (3.24).

Using standard elliptic estimates, we deduce pointwise bounds on $\left|v_{k}\right|$ and $\left|\nabla v_{k}\right|$ on the $s \geq-1$ part of the $i$ th end. It follows as in (2.9) that on the $s \geq 0$ part of the $i$ th end,

$$
\left|v_{k}\right|,\left|\nabla v_{k}\right| \leq c e^{-E_{k} r / 2}\left\|g_{k}\right\|_{2} e^{-E_{i} s} .
$$

A virtual repeat of the arguments that lead to (3.14) now proves (3.24).

\section{Genericity of immersion singularities}

This section is devoted to proving:

Theorem 4.1. If the admissible almost complex structure $J$ on $\mathbb{R} \times Y$ is generic, then all non-multiply-covered J-holomorphic curves of index $\leq 2$ are immersed.

Proof. The proof has nine steps.

Step 1 . We begin by setting up the deformation theory for $J$-holomorphic curves in $\mathbb{R} \times Y$ that are not necessarily immersed.

Fix an admissible almost complex structure $J$ on $\mathbb{R} \times Y$. Let $\varphi:(C, j) \rightarrow$ $\mathbb{R} \times Y$ be a $J$-holomorphic curve, with ends asymptotic to Reeb orbits as usual. Let $\mathcal{O}$ denote the set of Reeb orbits at which $C$ has ends. Recall that the domain $C$ is a punctured compact Riemann surface, and $j$ denotes the complex structure on $C$; thus the equation for $\varphi$ to be $J$-holomorphic can be written as

$$
\frac{1}{2}(d \varphi+J \circ d \varphi \circ j)=0
$$


The derivative of this equation with respect to deformations of $\varphi$ defines a real linear Fredholm operator

$$
D_{\varphi}: L_{1+}^{2}\left(C ; \varphi^{*} T X\right) \longrightarrow L_{0+}^{2}\left(C ; T^{0,1} C \otimes_{\mathbb{C}} \varphi^{*} T X\right),
$$

where $X:=\mathbb{R} \times Y$. Here the almost complex structure $J$ is used to regard $\varphi^{*} T X$ as a rank 2 complex vector bundle over $C$. Also $L_{k+}^{2}$ denotes the weighted $L_{k}^{2}$ space, using a weight which on the ends of $C$ is equal to $e^{\varepsilon|s|}$, where $\varepsilon>0$ is small with respect to the Reeb orbits in the finite set $\mathcal{O}$.

Observe that the diagram

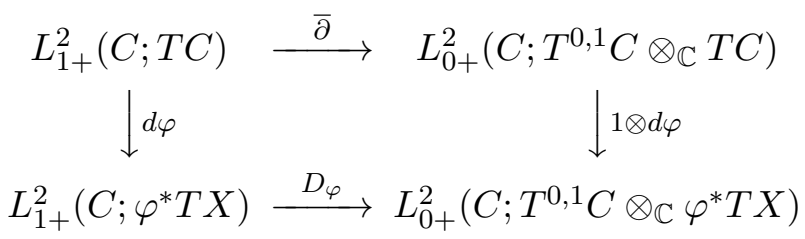

commutes. In particular, this is why weighted Sobolev spaces are needed to make the operator (4.2) Fredholm. Indeed, the weights are present so as to deal with the fact that the operator $\bar{\partial}$ on a cylinder is not Fredholm as a map from $L_{1}^{2}$ to $L^{2}$, because the operator $i \partial_{t}$ has zero modes on the circle. Note that the operator $\bar{\partial}$ in (4.3) has zero kernel, except when $C$ is a plane in which case the kernel has dimension 1 over $\mathbb{C}$.

Let $B$ be the image of a smooth embedding of a ball into the space of complex structures on $C$ with the following two properties: First, $j \in B$, and each $j^{\prime} \in B$ agrees with $j$ outside of a compact subset of $C$. Second, $T_{j} B$ projects isomorphically to the cokernel of the operator $\bar{\partial}$ in (4.3). This condition makes sense because a tangent vector $j \in T_{j} B$ defines a smooth, compactly supported bundle endomorphism of $T C$ satisfying $j \dot{j}+\dot{j} j=0$.

Differentiation of equation (4.1) with respect to deformations of $\varphi$ and $j$ gives rise to a Fredholm operator

$$
\widetilde{D}_{\varphi}: T_{j} B \oplus L_{1+}^{2}\left(C ; \varphi^{*} T X\right) \longrightarrow L_{0+}^{2}\left(C ; T^{0,1} C \otimes_{\mathbb{C}} \varphi^{*} T X\right)
$$

defined by

$$
\widetilde{D}_{\varphi}(\dot{j}, \dot{\varphi}):=D_{\varphi} \dot{\varphi}+\frac{1}{2} J \circ d \varphi \circ \dot{j} .
$$

When $C$ is a plane, we implicitly use (4.3) to regard $\widetilde{D}_{\varphi}$ instead as a Fredholm operator on $T_{j} B \oplus L_{1+}^{2}\left(C ; \varphi^{*} T X\right) / d \varphi(\operatorname{Ker}(\bar{\partial}))$. The curve $\varphi$ is said to be "unobstructed" if the operator $\widetilde{D}_{\varphi}$ is surjective. Standard arguments show that if $J$ is generic, then all non-multiply-covered $J$-holomorphic curves are unobstructed in this sense.

Step 2. We now explain why if $\varphi$ is unobstructed in the above sense, then the kernel of $\widetilde{D}_{\varphi}$ is naturally identified with the tangent space at $\varphi$ to the moduli space of $J$-holomorphic curves where $J$ is fixed. 
To define the correspondence, $\operatorname{let}(\dot{j}, \dot{\varphi}) \in \operatorname{Ker}\left(\widetilde{D}_{\varphi}\right)$. Since $\widetilde{D}_{\varphi}$ is surjective, the implicit function theorem can be used in the usual way to find a smooth family of $J$-holomorphic maps $\varphi_{t}:\left(C, j_{t}\right) \rightarrow \mathbb{R} \times Y$ parameterized by $t$ in a neighborhood of 0 , with $\left(j_{0}, \varphi_{0}\right)=(j, \varphi)$, satisfying the following two properties: First, $\left.\left(d j_{t} / d t\right)\right|_{t=0}=\dot{j}$. Second, $\varphi_{t}=\exp _{\varphi}\left(v_{t}\right)$ where exp : $T X \rightarrow X$ is the exponential map determined by some $\mathbb{R}$-invariant metric on $X$, while $v_{t}$ is a smooth $L_{1+}^{2}$ section of $\varphi^{*} T X$, and $\left.\left(d v_{t} / d t\right)\right|_{t=0}=\dot{\varphi}$. Since $\dot{\varphi}$ is in $L_{1+}^{2}$, the considerations in Section 2 show that there is a constant $c>0$ depending only on the Reeb orbits in the finite set $\mathcal{O}$, such that $|\dot{\varphi}| \leq e^{-c|s|}$ for large $|s|$. Consequently each curve $\varphi_{t}:\left(C, j_{t}\right) \rightarrow \mathbb{R} \times Y$ is still in the moduli space $\mathcal{M}^{J}$. There is then a well-defined map $\Phi: \operatorname{Ker}\left(\widetilde{D}_{\varphi}\right) \rightarrow T_{\varphi} \mathcal{M}^{J}$ sending $(\dot{j}, \dot{\varphi})$ to the derivative of the family of holomorphic curves $\varphi_{t}$ : $\left(C, j_{t}\right) \rightarrow \mathbb{R} \times Y$ with respect to $t$ at $t=0$.

To show that $\Phi$ is surjective, consider a smooth family of holomorphic curves $\varphi_{t}:\left(C, j_{t}\right) \rightarrow \mathbb{R} \times Y$ parameterized by $t$ in a neighborhood of 0 with $\left(j_{0}, \varphi_{0}\right)=(j, \varphi)$. By reparameterization of the holomorphic curves $\varphi_{t}$, we can arrange that each $j_{t}$ agrees with $j$ outside of a compact subset of $C$. This is because the pairs $\left(C, j_{t}\right)$ are punctured compact Riemann surfaces, and the complex structures on the corresponding closed surfaces are locally diffeomorphic near the punctures. Now define $j:=\left.\left(d j_{t} / d t\right)\right|_{t=0}$ and $\dot{\varphi}:=\left.\left(d \varphi_{t} / d t\right)\right|_{t=0}$. These are smooth sections over $C$ of $T^{0,1} C \otimes_{\mathbb{C}} T C$ and $T^{0,1} C \otimes_{\mathbb{C}} \varphi^{*} T X$, respectively. Differentiation of the equation $J \circ d \varphi_{t}=d \varphi_{t} \circ j_{t}$ with respect to $t$ at $t=0$ shows that

$$
D_{\varphi} \dot{\varphi}+\frac{1}{2} J \circ d \varphi \circ \dot{j}=0 .
$$

By construction, $\dot{j}$ is compactly supported. To describe the asymptotic behavior of $\dot{\varphi}$, note that on the ends of $C$, the map $\varphi$ is an immersion by $[\mathbf{1 3}$, so we have a spitting $\varphi^{*} T X=T C \oplus N$, where $N$ denotes the normal bundle to $C$, and the splitting is defined using an $\mathbb{R}$-invariant metric on $X$, which is preserved by $J$. By a further reparameterization of the holomorphic curves $\varphi_{t}$, we can arrange that the $T C$ component of $\dot{\varphi}$ is 0 on the ends of $C$. Meanwhile, the analysis from Section 2 shows that the $N$ component of $\dot{\varphi}$ is bounded from above by $e^{-c|s|}$ on the ends. So $\dot{j}$ and $\dot{\varphi}$ are in $L_{1+}^{2}$, provided that the constant $\varepsilon>0$ used to define the spaces $L_{k+}^{2}$ is chosen smaller than $c$. Since the projection of $T_{j} B$ to the cokernel of the operator $\bar{\partial}$ in (4.3) is surjective, there is a pair $(b, v) \in T_{j} B \oplus L_{1+}^{2}(C ; T C)$ such that

$$
\bar{\partial} v+b=\dot{j} .
$$

Since the diagram (4.3) commutes, it follows from (4.5) and (4.6) that

$$
\left(b, \dot{\varphi}+\frac{1}{2} j \bar{\partial} v\right) \in \operatorname{Ker}\left(\widetilde{D}_{\varphi}\right) .
$$


And $\Phi$ sends the above to the tangent vector $\left.\left(d\left(j_{t}, \varphi_{t}\right) / d t\right)\right|_{t=0}$.

To show that $\Phi$ is injective, let $(\dot{j}, \dot{\varphi}) \in \operatorname{Ker}\left(\widetilde{D}_{\varphi}\right)$, and suppose that the corresponding tangent vector to the moduli space is zero. This means that there is a smooth 1-parameter family of holomorphic curves $\varphi_{t}:\left(C, j_{t}\right) \rightarrow$ $\mathbb{R} \times Y$ parameterized by $t$ in a neighborhood of 0 with $\left(j_{0}, \varphi_{0}\right)=(j, \varphi)$ and $\left.\left(d j_{t} / d t\right)\right|_{t=0}=\dot{j}$ and $\left.\left(d \varphi_{t} / d t\right)\right|_{t=0}=\dot{\varphi}$, such that the holomorphic curves $\varphi_{t}$ are all equivalent to each other: that is, there is a 1-parameter family of diffeomorphisms $\psi_{t}: C \rightarrow C$ with $\varphi_{t}=\varphi \circ \psi_{t}$ and $d \psi_{t} \circ j_{t}=j \circ \psi_{t}$. Define $\eta:=\left.\left(d \psi_{t} / d t\right)\right|_{t=0}$; this is a smooth, $L_{1+}^{2}$ section of $T C$ satisfying $\dot{\varphi}=d \varphi(\eta)$. Since the diagram (4.3) commutes, and since $J \circ d \varphi=d \varphi \circ j$, the equation $\widetilde{D}_{\varphi}(\dot{j}, \dot{\varphi})$ becomes

$$
d \varphi \circ\left(\bar{\partial} \eta+\frac{1}{2} j \circ \dot{j}\right)=0
$$

Therefore,

$$
\bar{\partial}(2 j \eta)=\dot{j}
$$

wherever $d \varphi \neq 0$; and by continuity this equation holds on all of $C$. Since the projection of $T B$ to the cokernel of $\bar{\partial}$ is injective, it follows that $\dot{j}=0$ and $\bar{\partial} \eta=0$. Thus $(\dot{j}, \dot{\varphi})$ is equivalent to 0 .

Step 3. We now set up the proof of Theorem 4.1.

Fix an admissible $J$, and assume that $J$ is generic so that all non-multiplycovered $J$-holomorphic curves are unobstructed. Let $\varphi:(C, j) \rightarrow \mathbb{R} \times Y$ be a non-multiply-covered $J$-holomorphic curve of index $\leq 2$. Assume that $\varphi$ is not an immersion; in particular this implies that it is not $\mathbb{R}$-invariant. Define $B$ as in Step 1. Fix $\delta>0$ small and $l \gg 2$, and let $\mathcal{U}$ be a small neighborhood of $J$ in the space of $C^{l}$ admissible almost complex structures on $\mathbb{R} \times Y$ that agree with $J$ where the distance is less than $\delta$ to any of the Reeb orbits corresponding to the ends of $C$. There is a smooth, universal family $\mathcal{C} \rightarrow \mathcal{U}$ whose fiber over $J^{\prime} \in \mathcal{U}$ consists of pairs $\left(j^{\prime}, \varphi^{\prime}\right)$, where $j^{\prime} \in B$ and $\varphi^{\prime}:\left(C, j^{\prime}\right) \rightarrow \mathbb{R} \times Y$ is a $J^{\prime}$-holomorphic map near $\varphi$, which is the composition of the exponential map with an $L_{2+}^{2}$ section of $\varphi^{*} T X$. When $C$ is a plane, we require the latter section to be $L^{2}$-orthogonal to $d \varphi(\operatorname{Ker}(\bar{\partial}))$. We can choose the neighborhood $\mathcal{U}$ to be small enough so that the fiber of $\mathcal{C}$ over each $J^{\prime} \in \mathcal{U}$ consists of unobstructed curves, and in particular is a manifold of dimension $\leq 2$. Note that $\mathbb{R}$ acts freely on $\mathcal{C}$, by composing $\varphi^{\prime}$ with translations of $\mathbb{R} \times Y$; and the projection to $\mathcal{U}$ is invariant under this action.

Let $u_{0} \in C$ be a point where $\varphi$ is not an immersion; since $\varphi$ is $J$ holomorphic, this means that the differential $d \varphi_{u_{0}}=0$. Let $D \subset C$ be a small disc containing $u_{0}$, such that $\varphi$ is an embedding on the closure of $D$ minus the origin. Let $\mathcal{Z} \subset \mathcal{C} \times D$ denote the locus of points $\left(J^{\prime}, j^{\prime}, \varphi^{\prime}, u\right) \in \mathcal{C} \times D$ such that $d \varphi_{u}^{\prime}=0$. Note that the $\mathbb{R}$-action on $\mathcal{C}$, crossed with the identity on $D$, sends $\mathcal{Z}$ to itself. 
To prove Theorem 4.1, we will show that if $\delta$ is sufficiently small, then $\mathcal{Z}$ is a codimension 4 submanifold of $\mathcal{C} \times D$. Granted this, then $\mathcal{Z} / \mathbb{R}$ is a codimension 4 submanifold of $(\mathcal{C} / \mathbb{R}) \times D$. Since the fibers of the projection $(\mathcal{C} / \mathbb{R}) \times D \rightarrow \mathcal{U}$ have dimension at most 3, the usual Sard-Smale argument can be used to deduce that there is an open dense subset of $\mathcal{U}$ whose inverse image in $\mathcal{C} \times D$ is disjoint from $\mathcal{Z}$. As in the beginning of the proof of Proposition 3.2, it then follows that there is a Baire set of admissible almost complex structures satisfying the condition in Theorem 4.1.

Step 4. To prepare for the proof that $\mathcal{Z}$ is a codimension 4 submanifold of $\mathcal{C} \times D$, we now choose convenient local coordinates $(s, t, x, y)$ on a neighborhood of $\varphi\left(u_{0}\right)$ in $\mathbb{R} \times Y$. These coordinates will be defined for $|t|,|x|,|y|$ small, and will have the following properties:

(1) $\varphi\left(u_{0}\right)$ corresponds to $(0,0,0,0)$.

(2) $\partial_{s}$ is the derivative of the $\mathbb{R}$-action on $\mathbb{R} \times Y$, and $J \partial_{s}=\partial_{t}$.

(3) $\partial_{x}$ and $\partial_{y}$ are tangent to the contact plane field at $(x, y)=(0,0)$.

(4) $J \partial_{x}=\partial_{y}$ at $t=0$.

To find such coordinates, first note that there exists a $J$-holomorphic embedding $\imath: \Delta \subset \mathbb{R} \times Y$, where $\Delta$ is a neighborhood of the origin in $\mathbb{C}$, such that $\imath(0)=\varphi\left(u_{0}\right)$, and $d \imath_{0}$ maps $T_{0} \mathbb{C}$ to the contact plane field at $\varphi\left(u_{0}\right)$. Write the holomorphic coordinate on $\Delta$ as $w=x+i y$. The desired coordinates are now described by a map

$$
\Phi: \mathbb{R} \times\left(-\delta_{0}, \delta_{0}\right) \times \Delta \longrightarrow \mathbb{R} \times Y,
$$

where $\Phi(s, t, w)$ is obtained by starting with the point $\imath(w) \in \mathbb{R} \times Y$, translating the $\mathbb{R}$ coordinate by $s$, and then flowing along the Reeb vector field $\mathbf{R}$ for time $t$. If $\delta_{0}>0$ is small, then $\Phi$ is an embedding.

These coordinates satisfy property (1), and the first part of property (2), by construction. The second part of property (2) holds because the admissible almost complex structure $J$ is required to satisfy $J \partial_{s}=\mathbf{R}$. Property (3) holds for $t=0$ by construction, and for general $t$ because the Lie derivatives with respect to $\mathbf{R}$ of $\partial_{x}, \partial_{y}$, and the contact form all vanish. Property (4) holds for $s=0$ by construction, and for general $s$ because $J$ is $\mathbb{R}$-invariant.

Step 5. We now write down equations for $\mathcal{Z}$ to be a codimension 4 submanifold of $\mathcal{C} \times D$.

To start, in the coordinate chart from Step 4 , if we write $z:=s+i t$, then a basis for the $J$ version of $T^{1,0}(\mathbb{R} \times Y)$ is given by

$$
e_{0}=d z+\gamma d \bar{w}, \quad e_{1}=d w+\sigma d \bar{w}
$$

where $\gamma$ and $\sigma$ vanish at $t=0$. Likewise, if $J^{\prime} \in \mathcal{U}$, then because $J^{\prime} \partial_{s}=\partial_{t}$, a basis for the $J^{\prime}$ version of $T^{1,0}(\mathbb{R} \times Y)$ is given by

$$
e_{0}^{\prime}=d z+\gamma^{\prime} d \bar{w}, \quad e_{1}^{\prime}=d w+\sigma^{\prime} d \bar{w},
$$

where $\gamma^{\prime}$ and $\sigma^{\prime}$ no longer necessarily vanish at $t=0$. 
Next, let us choose the set $B$ of complex structures on $C$ from Step 1 so that all $j^{\prime} \in B$ agree with $j$ on the disc $D \subset C$. Also, choose $D$ sufficiently small so that $\varphi(D)$ is contained in the coordinate chart from Step 4. Fix a holomorphic local coordinate $u$ on $D$, with $u=0$ corresponding to $u_{0}$. Now let $\left(J^{\prime}, j^{\prime}, \varphi^{\prime}\right) \in \mathcal{C}$. Then the equations for $\varphi^{\prime}$ to be $J^{\prime}$-holomorphic on $D$ can be written as follows. On $D$, in our local coordinates, write $\varphi^{\prime}(u)=\left(z^{\prime}(u), w^{\prime}(u)\right)$. Then $\varphi^{\prime}$ is $J^{\prime}$-holomorphic on $D$ if and only if it pulls back $e_{0}^{\prime}$ and $e_{1}^{\prime}$ to multiples of $d u$, which means that

$$
\bar{\partial} z^{\prime}+\gamma^{\prime} \overline{\partial w^{\prime}}=0, \quad \bar{\partial} w^{\prime}+\sigma^{\prime} \overline{\partial w^{\prime}}=0 .
$$

Here $\gamma^{\prime}$ and $\sigma^{\prime}$ are shorthand for $\left(\varphi^{\prime}\right)^{*} \gamma^{\prime}$ and $\left(\varphi^{\prime}\right)^{*} \sigma^{\prime}$, respectively.

It follows using (4.8) that $\mathcal{Z}$ is the zero locus of the smooth function $f: \mathcal{C} \times D \rightarrow \mathbb{C}^{2}$ defined by

$$
f\left(J^{\prime}, j^{\prime}, \varphi^{\prime}, u\right):=\left.\left(\partial_{u} z^{\prime}+\gamma^{\prime} \partial_{u} \overline{w^{\prime}}, \partial_{u} w^{\prime}+\sigma^{\prime} \partial_{u} \overline{w^{\prime}}\right)\right|_{u} .
$$

Thus to prove that $\mathcal{Z}$ is a codimension 4 submanifold of $\mathcal{C} \times D$ near our given point $(J, j, \varphi, 0) \in \mathcal{Z}$, by the implicit function theorem it is enough to show that the differential

$$
d f_{(J, j, \varphi, 0)}: T_{(J, j, \varphi, 0)}(\mathcal{C} \times D) \longrightarrow \mathbb{C}^{2}
$$

is surjective.

Step 6. We now give an explicit description of the tangent space $T_{(J, j, \varphi)} \mathcal{C}$ and the differential (4.10), in preparation for showing that (4.10) is surjective.

In the notation from Step 1, a tangent vector in $T_{(J, j, \varphi)} \mathcal{C}$ is equivalent to a triple $(\dot{J}, \dot{j}, \dot{\varphi})$, where:

(i) $\dot{J}$ is a $C^{l}$ bundle endomorphism of $T X$ which satisfies $J \dot{J}+\dot{J} J=0$, annihilates $\partial_{s}$ and $\mathbf{R}$ and maps the contact plane field $\xi$ to itself.

(ii) $\dot{j}$ is a smooth, compactly supported bundle endomorphism of $T C$ satisfying $j \dot{j}+\dot{j} j=0$.

(iii) $\dot{\varphi}$ is an $L_{1+}^{2}$ section of $\varphi^{*} T X$ over $C$ satisfying

$$
D_{\varphi} \dot{\varphi}+\frac{1}{2}(\dot{J} \circ d \varphi \circ j+J \circ d \varphi \circ \dot{j})=0 .
$$

When $C$ is a plane, we declare two such sections $\dot{\varphi}$ to be equivalent if their difference is in $d \varphi(\operatorname{Ker}(\bar{\partial}))$.

(iv) $\dot{j} \in T_{j} B$.

(v) $\dot{J}$ is invariant under $\mathbb{R}$ translation and vanishes within distance $\delta$ of the Reeb orbits in $\mathcal{O}$.

Of course condition (ii) follows from condition (iv); but our construction later will obtain condition (ii) before obtaining condition (iv).

In the local coordinates from Step 4, these data appear as follows. By condition (i) above, $\dot{J}$ determines a pair of functions $(\dot{\gamma}, \dot{\sigma})$, which describe 
the respective changes in $\gamma$ and $\sigma$. Likewise, $\dot{\varphi}$ determines a pair of functions $(\dot{z}, \dot{w})$ on $D$. With this notation, Equation (4.11) on $D$ is equivalent to the equations obtained by differentiating (4.8), which have the form

$$
\begin{aligned}
\bar{\partial} \dot{z}+\gamma \bar{\partial} \bar{w}+e_{\gamma}(\dot{z}, \dot{w}) & =-\dot{\gamma} \bar{\partial} \bar{w} \\
\bar{\partial} \dot{w}+\sigma \bar{\partial} \bar{w}+e_{\sigma}(\dot{z}, \dot{w}) & =-\dot{\sigma} \bar{\partial} \bar{w} .
\end{aligned}
$$

Here $e_{\gamma}$ and $e_{\sigma}$ are linear functions of $\dot{z}$ and $\dot{w}$, arising from the derivatives of $\gamma$ and $\sigma$; and $(z, w)$ denotes the pair of functions determined by $\varphi$.

In the above notation, it follows from (4.9), with the help of (4.8), that the differential (4.10) is given as follows: If $\dot{u} \in T_{0} D$, then

$$
d f_{(J, j, \varphi, 0)}(\dot{J}, \dot{j}, \dot{\varphi}, \dot{u})=\left.\left(\partial_{u} \dot{z}, \partial_{u} \dot{w}\right)\right|_{u=0}+\left.\left(\partial_{u} \partial_{u} z, \partial_{u} \partial_{u} w\right)\right|_{u=0} d u(\dot{u})
$$

To prove that the differential (4.10) is surjective, we will show that for any $\eta=\left(\eta_{z}, \eta_{w}\right) \in \mathbb{C}^{2}$, there exists $(\dot{J}, \dot{j}, \dot{\varphi}) \in T_{(J, j, \varphi)} \mathcal{C}$ and $\dot{u} \in T_{0} D$ such that

$$
\left.\left(\partial_{u} \dot{z}, \partial_{u} \dot{w}\right)\right|_{u=0}+\left.\left(\partial_{u} \partial_{u} z, \partial_{u} \partial_{u} w\right)\right|_{u=0} d u(\dot{u})=\left(\eta_{z}, \eta_{w}\right) .
$$

Step 7 . We now begin the construction of $(\dot{J}, \dot{j}, \dot{\varphi}) \in T_{(J, j, \varphi)} \mathcal{C}$ and $\dot{u} \in T_{0} D$ satisfying (4.14).

Lemma 4.2. There exist constants $\rho_{0}>0$ and $c$ such that if $D$ has radius $\rho<\rho_{0}$, then given $\eta=\left(\eta_{z}, \eta_{w}\right) \in \mathbb{C}^{2}$, there exist smooth functions $\dot{z}, \dot{w}$ : $D \rightarrow \mathbb{C}$ such that:

- Equations (4.12) holds with $\dot{\gamma}=\dot{\sigma}=0$.

- Equation (4.14) holds with $\dot{u}=0$.

Proof. We will first solve (4.12) with $\dot{\gamma}=\dot{\sigma}=0$, and then explain how to solve (4.14) with $\dot{u}=0$ as well.

Write $\zeta:=(\dot{z}, \dot{w})$ and $\eta:=\left(\eta_{z}, \eta_{w}\right)$. The disc of radius $\rho$ can be identified with a disc of radius 1 so that on the disc of radius 1, Equation (4.12) with $\dot{\gamma}=\dot{\sigma}=0$ has the form

$$
\bar{\partial}_{u} \zeta+\Theta \zeta=0
$$

where $\Theta$ is $\mathbb{R}$-linear and satisfies $|\Theta|,|d \Theta|<c \rho$. It is enough to solve this equation on the disc of radius $1 / 2$, for which purpose we can assume that $\Theta$ vanishes outside of the disc of radius $3 / 4$ and has derivative bounded by $c \rho$. Now write $\zeta=\zeta_{0}+\Delta$, where $\zeta_{0}:=\eta u$; then Equation (4.15) becomes

$$
\bar{\partial}_{u} \Delta+\Theta \Delta+\Theta \zeta_{0}=0 .
$$

If $\rho>0$ is sufficiently small, then the contraction mapping theorem finds a unique continuous function $\Delta$ on $D$ satisfying

$$
\Delta(u)=\left.\frac{1}{\pi} \int_{|v| \leq 1} \frac{1}{u-v}\left(\Theta \Delta+\Theta \zeta_{0}\right)\right|_{v} d^{2} v
$$


and this $\Delta$ will satisfy (4.16). Moreover, it follows from (4.17) that

$$
|\Delta(u)| \leq c \rho(\sup |\Delta|+|\eta|),
$$

where $|\eta|:=\sqrt{\left|\eta_{z}\right|^{2}+\left|\eta_{w}\right|^{2}}$. Thus

$$
|\Delta(u)| \leq c \rho|\eta|(1-c \rho)^{-1} .
$$

Differentiating (4.17), and using (4.18) and our assumptions on $\Theta$, gives a similar bound on $\left|\partial_{u} \Delta\right|$. As a result, if $\rho>0$ is sufficiently small then

$$
\left|\partial_{u} \zeta\right|_{u=0}-\eta\left|\leq \frac{1}{2}\right| \eta \mid \text {. }
$$

Since the set

$$
\left\{\left.\partial_{u} \zeta\right|_{u=0} \mid \zeta \text { solves }(4.15)\right\}
$$

is a real linear subspace of $\mathbb{C}^{2}$, it follows that this set is all of $\mathbb{C}^{2}$. This proves the lemma.

Now fix a smooth function $\beta: \mathbb{C} \rightarrow[0,1]$ with $\beta(u)=0$ for $|u| \geq 1$ and $\beta(u)=1$ for $|u| \leq 1 / 2$. Given $\rho>0$, define $\beta_{\rho}: \mathbb{C} \rightarrow[0,1]$ by $\beta_{\rho}(u):=\beta\left(\rho^{-1} u\right)$.

Next, given $\eta=\left(\eta_{z}, \eta_{w}\right) \in \mathbb{C}^{2}$, let $\rho>0$ be small, and let $\left(\dot{z}_{\eta}, \dot{w}_{\eta}\right)$ denote the pair of functions provided by Lemma 4.2. Take

$$
\dot{z}:=\beta_{\rho} \dot{z}_{\eta}, \quad \dot{w}:=\beta_{\rho} \dot{w}_{\eta},
$$

and extend these to a section $\dot{\varphi}$ of $\varphi^{*} T X$ over $C$ by declaring $\dot{\varphi}$ to be zero on the complement of $D$. Then over the disc of radius $\rho / 2$, equation (4.11) holds with $\dot{J}=\dot{j}=0$.

Let $D_{\rho}$ denote the portion of $D$ where the radius is between $\rho / 2$ and $\rho$. If $\rho$ is sufficiently small, then the restriction of $\varphi$ to $D_{\rho}$ does not hit the finite set of points in $C$ where $\varphi(C)$ is tangent to $\xi$ or to the span of vectors $\partial_{s}$ and $\mathbf{R}$. It then follows as in the proof of Lemma 3.5, Step 2, that there exist $\dot{J}$ and $\dot{j}$ such that:

- the quadruple $(\dot{J}, \dot{j}, \dot{\varphi}, 0)$ satisfies conditions (i) to (iii) above, as well as equation (4.14).

- $\dot{j}$ and the restriction of $\dot{J}$ to $C$ are supported in $D_{\rho}$.

It proves convenient later to also choose $\rho$ sufficiently small so that:

- the restriction of $\varphi$ to $D_{\rho}$ does not hit the finite set of points in $C$ where $\varphi(C)$ intersects the Reeb orbits in $\mathcal{O}$.

Step 8. We now modify the quadruple $(\dot{J}, \dot{j}, \dot{\varphi}, 0)$ so as to also satisfy condition (iv), namely that $\dot{j} \in T_{j} B$, while still satisfying conditions (i) to (iii) and equation (4.14).

The idea here is that one could regard $f$ as a function defined on a larger space, where the complex structure on $C$ is not required to be in $B$, such that $f$ is invariant under an appropriate equivalence relation; then by moving 
along an appropriate slice in this larger space we can obtain $j \in T_{j} B$ without changing $d f$. This works concretely as follows. By definition, $T_{j} B$ projects isomorphically onto the cokernel of the operator

$$
\bar{\partial}: L_{1+}^{2}(C ; T C) \longrightarrow L_{0+}^{2}\left(C ; T^{0,1} C \otimes_{\mathbb{C}} T C\right) .
$$

Hence there exists a tangent vector $\dot{j}_{0} \in T_{j} B$, and a section $\zeta \in L_{1+}^{2}(C ; T C)$, such that

$$
2 j \circ \bar{\partial} \zeta+\dot{j}_{0}=\dot{j}
$$

as bundle automorphisms of $T C$. Let $\dot{\varphi}_{0}:=d \varphi \circ \zeta$. To achieve condition (iv), replace the quadruple $(\dot{J}, \dot{j}, \dot{\varphi}, 0)$ with

$$
\left(\dot{J}, \dot{j}_{0}, \dot{\varphi}+\dot{\varphi}_{0},-\zeta(0)\right) .
$$

We need to check that the new quadruple (4.20) still satisfies equations (4.11) and (4.14).

To verify equation (4.11), use the commutativity of the diagram (4.3), the fact that $\varphi$ is $J$-holomorphic and equation (4.19), to find that

$$
\begin{aligned}
D_{\varphi}\left(\dot{\varphi}_{0}\right) & =d \varphi \circ \bar{\partial} \zeta \\
& =-J \circ d \varphi \circ j \bar{\partial} \zeta \\
& =J \circ d \varphi \circ \frac{1}{2}\left(\dot{j}_{0}-\dot{j}\right) .
\end{aligned}
$$

It follows from this that the new quadruple still satisfies equation (4.11).

To prove that the new quadruple (4.20) still satisfies equation (4.14), write $\dot{\varphi}_{0}=\left(\dot{z}_{0}, \dot{w}_{0}\right)$ on $D$. We need to show that

$$
\left.\left(\partial_{u} \dot{z}_{0}, \partial_{u} \dot{w}_{0}\right)\right|_{u=0}=\left.\left(\partial_{u} \partial_{u} z, \partial_{u} \partial_{u} w\right)\right|_{u=0} d u(\zeta(0)) .
$$

It follows from the definition of $\dot{\varphi}_{0}$ and equation (4.8) that

$$
\left(\dot{z}_{0}, \dot{w}_{0}\right)=\left(\partial_{u} z, \partial_{u} w\right) d u(\zeta)-\left(\gamma \partial_{\bar{u}} \bar{w}, \sigma \partial_{\bar{u}} \bar{w}\right) d \bar{u}(\zeta) .
$$

Since $\partial_{u} z, \partial_{u} w, \gamma$ and $\sigma$ all vanish at $u=0$, equation (4.21) follows.

Step 9. The previous steps constructed a quadruple $(\dot{J}, \dot{j}, \dot{\varphi}, \dot{u})$ satisfying conditions (i) to (iv) and equation (4.14); we now modify this quadruple so as to also satisfy condition (v).

Recall from Step 7 that the restriction of $\dot{J}$ to $\varphi(C)$, call it $\dot{J}_{C}$, is supported inside $D_{\rho}$. Only $\dot{J}_{C}$ enters into equation (4.11); so we just need to modify $\dot{J}_{C}$ (while changing the other data $\dot{j}, \dot{\varphi}, \dot{u}$ as appropriate) so that $\dot{J}_{C}$ has an extension over $X$ satisfying conditions (i) and (v).

For this purpose, let $\Lambda \subset D$ denote the set of points $u \in D$ such that $\varphi(u)$ intersects the $s \mapsto s-s_{0}$ translate of $\varphi$ for some $s_{0} \in[-\infty,+\infty] \backslash\{0\}$. As in the proof of Lemma 3.5, Step 2, $\Lambda$ is a closed codimension 1 subvariety of $D$. Let $\varepsilon>0$ be small, and let $\chi: D \rightarrow[0,1]$ be a smooth function which is 1 where the distance to $\Lambda$ is $\geq 2 \varepsilon$ and 0 where the distance to $\Lambda$ is $\leq \varepsilon$. 
We now replace $\dot{J}_{C}$ by $\chi \dot{J}_{C}$. Note that $\chi \dot{J}_{C}$ still satisfies condition (i) on $D$, because condition (i) is a system of homogeneous linear equations for $\dot{J}$. Furthermore, if $\delta>0$ is sufficiently small, then $\chi \dot{J}_{C}$ has an extension over $X$ satisfying (i) and (v); pick such an extension and call it $\dot{J}^{\prime}$.

We now modify $\dot{j}$ and $\dot{\varphi}$ to restore equation (4.11). Since the operator $\widetilde{D}_{\varphi}$ is surjective, there exists $\dot{j}_{1} \in T_{j} B$, and an $L_{1+}^{2}$ section $\dot{\varphi}_{1}$ of $\varphi^{*} T X$, such that

$$
D_{\varphi} \dot{\varphi}_{1}+\frac{1}{2} J \circ d \varphi \circ \dot{j}_{1}=(\chi-1) \dot{J}_{C} .
$$

Moreover, these can be chosen so that

$$
\left\|\dot{\varphi}_{1}\right\|_{L_{1}^{2}} \leq c\left\|(\chi-1) \dot{J}_{C}\right\|_{L^{2}}
$$

where $c$ is a constant which does not depend on $\varepsilon$.

It follows from (4.22) that if we define $\dot{j}^{\prime}:=\dot{j}+\dot{j}_{1}$ and $\dot{\varphi}^{\prime}:=\dot{\varphi}+\dot{\varphi}_{1}$, then the triple $\left(\dot{J}^{\prime}, \dot{j}^{\prime}, \dot{\varphi}^{\prime}\right)$ satisfies equation (4.11), and hence all of the conditions (i) to $(\mathrm{v})$.

The quadruple $\left(\dot{J}^{\prime}, \dot{j}^{\prime}, \dot{\varphi}^{\prime}, \dot{u}\right)$ might not satisfy equation (4.14). Rather, if we define

$$
\left(\eta_{z}^{\prime}, \eta_{w}^{\prime}\right):=d f_{(J, j, \varphi, 0)}\left(\dot{J}^{\prime}, \dot{j}^{\prime}, \dot{\varphi}^{\prime}, \dot{u}\right)
$$

and if we write $\dot{\varphi}_{1}=\left(\dot{z}_{1}, \dot{w}_{1}\right)$ on $D$, then it follows from equation (4.13) that

$$
\left(\eta_{z}^{\prime}, \eta_{w}^{\prime}\right)-\left(\eta_{z}, \eta_{w}\right)=\left.\left(\partial_{u} \dot{z}_{1}, \partial_{u} \dot{w}_{1}\right)\right|_{u=0} .
$$

To handle this discrepancy, note that by taking $\varepsilon$ sufficiently small, we can make the support of $(\chi-1) \dot{J}_{C}$ have arbitrarily small measure, and so by (4.23) we can make $\dot{\varphi}_{1}$ have arbitrarily small $L_{1}^{2}$ norm. It then follows by elliptic regularity, as in Lemma 2.2 , that we can make the expression in (4.24) have arbitrarily small norm.

We conclude from the above discussion that for any nonzero $\left(\eta_{z}, \eta_{w}\right) \in \mathbb{C}^{2}$, we can find $(\dot{J}, \dot{j}, \dot{\varphi}, \dot{u}) \in T_{(J, j, \varphi, 0)}(\mathcal{C} \times D)$ such that

$$
\left|d f_{(J, j, \varphi, 0)}(\dot{J}, \dot{j}, \dot{\varphi}, \dot{u})-\left(\eta_{z}, \eta_{w}\right)\right| \leq \frac{1}{2}\left|\left(\eta_{z}, \eta_{w}\right)\right| .
$$

Since $d f_{(J, j, \varphi, 0)}$ is linear, it follows that it is surjective. This completes the proof of Theorem 4.1.

\section{The gluing construction}

As in Section 1.2, fix a generic $J$ such that all non-multiply-covered $J$ holomorphic curves are unobstructed, and let $\left(U_{+}, U_{-}\right)$be a gluing pair as in Definition I.1.9 satisfying the additional assumptions (1.1) and (1.2). Let $\mathcal{M}$ denote the moduli space of branched covers of $\mathbb{R} \times S^{1}$ as specified in (1.3). 
In this section, we explain a construction for gluing $U_{+}$and $U_{-}$to a $J$-holomorphic curve by patching an element of $\mathcal{M}$ between them. This procedure finds such a gluing for each zero of a certain section $\mathfrak{s}$ of the obstruction bundle $\mathcal{O}$ from Section I.2.3 over a certain open subset of $\mathbb{R}^{2} \times \mathcal{M}$. As a result, we obtain a "gluing map" $G$ from $\mathfrak{s}^{-1}(0)$ to the moduli space of $J$-holomorphic curves $\mathcal{M}^{J}\left(\alpha_{+}, \alpha_{-}\right)$.

\subsection{Preliminaries.}

(i) It follows from the definition of gluing pair that $U_{ \pm}$consists of an immersed, non-multiply-covered, index 1 component $u_{ \pm}$, together with a union $v_{ \pm}$of unbranched covers of $\mathbb{R}$-invariant cylinders. Index the negative ends of $U_{+}$such that the negative ends of $u_{+}$are indexed by $1, \ldots, \bar{N}_{+}$, while the negative ends of $v_{+}$are indexed by $\bar{N}_{+}+1, \ldots, N_{+}$. Similarly, index the positive ends of $U_{-}$such that the positive ends of $u_{-}$ are indexed by $-1, \ldots,-\bar{N}_{-}$, while the positive ends of $v_{-}$are indexed by $-\bar{N}_{-}-1, \ldots,-N_{-}$. (In fact Lemma I.3.7 implies that $\bar{N}_{+} \geq N_{+}-1$ and $\bar{N}_{-} \geq N_{-}-1$, but we will not need this.)

(ii) Fix an "exponential map" $e: \mathbb{R} \times S^{1} \times D \rightarrow \mathbb{R} \times Y$ for $\alpha$ as given by Lemma 2.1. This $e$ defines coordinates $(s, t, w)$ on a tubular neighborhood of $\mathbb{R} \times \alpha$ in $\mathbb{R} \times Y$. Fix $\delta_{0}>0$ sufficiently small so that $D$ contains the disc of radius $4 \delta_{0}$. By translating $U_{+}$upward, we may assume that for $i=1, \ldots, \bar{N}_{+}$, the $i$ th negative end of $u_{+}$is described in these coordinates by a map

$$
\begin{aligned}
(-\infty, 0] \times \widetilde{S^{1}} & \longrightarrow \mathbb{R} \times S^{1} \times \mathbb{C}, \\
(s, \tau) & \longmapsto\left(s, t, \eta_{i}(s, \tau)\right),
\end{aligned}
$$

where $\widetilde{S^{1}}$ denotes the $a_{i}$-fold cover of $S^{1} ; t$ denotes the projection of $\tau$; and $\left|\eta_{i}\right|<\delta_{0}$. Likewise, by translating $U_{-}$downward, we may assume that for $i=-1, \ldots,-\bar{N}_{-}$, the $i$ th positive end of $u_{-}$is described by a map

$$
\begin{aligned}
{[0, \infty) \times \widetilde{S^{1}} } & \longrightarrow \mathbb{R} \times S^{1} \times \mathbb{C}, \\
(s, \tau) & \longmapsto\left(s, t, \eta_{i}(s, \tau)\right),
\end{aligned}
$$

where $\widetilde{S^{1}}$ denotes the $a_{i}$-fold cover of $S^{1}$, and $\left|\eta_{i}\right|<\delta_{0}$.

Next, as in Lemma 2.1, choose an "exponential map" $e_{-}$, from a small radius disc bundle in the normal bundle of $u_{-}$to $\mathbb{R} \times Y$, with the following properties. First, $e_{-}$is an immersion; on the zero section $e_{-}$agrees with $u_{-}$; and on each fiber disc $u_{-}$is a $J$-holomorphic embedding. The constant $\delta_{0}$ above should be chosen sufficiently small so that $e_{-}$is defined on the radius $\delta_{0}$ disc bundle. In addition, on the positive ends of $u_{-}$, we require $e_{-}$to be compatible with $e$ in the following sense: For $i=-1, \ldots,-\bar{N}_{-}$, in the notation of $(5.2)$, use $(s, \tau)$ as coordinates on the $i$ th positive end of $u_{-}$, and use the coordinate $w$ to trivialize the normal bundle to the $i$ th positive end 
of $u_{-}$. Then the compatibility requirement is that if $|v|<\delta_{0}$ then

$$
e_{-}((s, \tau), v)=e\left(s, t, \eta_{i}(s, \tau)+v\right) .
$$

Choose an analogous exponential map $e_{+}$from the radius $\delta_{0}$ disc bundle in the normal bundle of $u_{+}$to $\mathbb{R} \times Y$.

(iii) Given a branched cover $\pi: \Sigma \rightarrow \mathbb{R} \times S^{1}$ in $\mathcal{M}$, let $\Lambda \subset \Sigma$ denote the union of the components of the level sets of $\pi^{*} s$ on $\Sigma$ that contain ramification points. For $i=1, \ldots, \bar{N}_{+}$, the $i$ th positive end of $\Sigma$ corresponds to a component of $\Sigma \backslash \Lambda$, which the asymptotic marking identifies with $\left(s_{i}-1, \infty\right) \times \mathbb{R} / 2 \pi a_{i} \mathbb{Z}$ for some real number $s_{i}$. Likewise, for $i=-1, \ldots,-\bar{N}_{-}$, the $i$ th negative end of $\Sigma$ corresponds to a component of $\Sigma \backslash \Lambda$, which the asymptotic marking identifies with $\left(-\infty, s_{i}+1\right) \times \mathbb{R} / 2 \pi a_{i} \mathbb{Z}$. Let $s_{+}:=\max _{i>0}\left\{s_{i}\right\}$ and $s_{-}:=\min _{i<0}\left\{s_{i}\right\}$. Note that $s_{i}, s_{+}$and $s_{-}$define functions on $\mathcal{M}$ which are continuous but not smooth. It proves convenient later to replace these functions by smooth functions which have $C^{0}$-distance less than $1 / 2$ from the original functions. We denote these smoothings by the same symbols.

(iv) The gluing construction requires fixing two constants $0<h<1$ and $r>h^{-1}$ which enter into the definitions of the relevant cutoff functions. The gluing construction will work for any $0<h<1$, as long as $r$ is sufficiently large with respect to $h$ in a sense to be explained below. (In Section 8 we will choose $h$ small in order to obtain good estimates on the nonlinear part of the obstruction section whose zero set characterizes the possible gluings.)

Throughout this section, the letter ' $c$ ' denotes a constant which depends only on $U_{+}$and $U_{-}$, and whose value may change from one appearance to the next.

5.2. Pregluing. With $r$ and $h$ fixed, the "gluing parameters" consist of a branched cover $\pi: \Sigma \rightarrow \mathbb{R} \times S^{1}$ in $\mathcal{M}$, together with real numbers $T_{+}, T_{-} \geq$ $5 r$. Given $T_{+}, T_{-}$and $\Sigma$, we now define the " $\left(T_{+}, T_{-}\right)$-pregluing" of $U_{+}$and $U_{-}$along $\Sigma$. This will be a map $u_{*}: C_{*} \rightarrow \mathbb{R} \times Y$.

To define the domain $C_{*}$, let $\Sigma^{\prime} \subset \Sigma$ be obtained from $\Sigma$ by removing the $s>s_{+}+T_{+}$portion of the positive ends indexed by $1, \ldots, \bar{N}_{+}$and the $s<s_{-}-T_{-}$portion of the negative ends indexed by $-1, \ldots,-\bar{N}_{-}$. Let $u_{+T}$ denote the $s \mapsto s+s_{+}+T_{+}$translate of $u_{+}$, and let $u_{+T}^{\prime}$ denote the $s \geq s_{+}+T_{+}$portion of $u_{+T}$. Let $u_{-T}$ denote the $s \mapsto s+s_{-}-T_{-}$translate of $u_{-}$, and let $u_{-T}^{\prime}$ denote the $s \leq s_{-}-T_{-}$portion of $u_{-T}$.

Let $C_{ \pm T}^{\prime}$ denote the domain of $u_{ \pm T}^{\prime}$. The domain $C_{*}$ is the quotient of $C_{+T}^{\prime} \sqcup \Sigma^{\prime} \sqcup C_{-T}^{\prime}$ obtained by identifying the $i$ th negative boundary circle of $C_{+T}^{\prime}$ with the $i$ th positive boundary circle of $\Sigma^{\prime}$ for $i=1, \ldots, \bar{N}_{+}$, and the $i$ th positive boundary circle of $C_{-T}^{\prime}$ with the $i$ th negative boundary circle of $\Sigma^{\prime}$ for $i=-1, \ldots,-\bar{N}_{-}$. The identification maps are well defined, because the asymptotic markings of the ends of $\Sigma$ and $u_{ \pm}$fix an identification of each 
such boundary circle with $\mathbb{R} / 2 \pi m \mathbb{Z}$, where $m$ is the covering multiplicity of the associated end.

For $i=1, \ldots, \bar{N}_{+}$, let $\Sigma_{i} \subset \Sigma^{\prime}$ denote the cylinder consisting of the $s_{i} \leq s \leq s_{+}+T_{+}$part of the $i$ th positive end of $\Sigma$. As above, the cylinder $\Sigma_{i}$ can also be naturally identified with the $s_{i} \leq s \leq s_{+}+T_{+}$portion of the $i$ th negative end of $u_{+T}$. For $i=-1, \ldots,-\bar{N}_{-}$, let $\Sigma_{i} \subset \Sigma^{\prime}$ denote the cylinder consisting of the $s_{-}-T_{-} \leq s \leq s_{i}$ part of the $i$ th negative end of $\Sigma$. This can also be identified with the corresponding portion of the $i$ th positive end of $u_{-T}$.

Fix a smooth function $\beta: \mathbb{R} \rightarrow[0,1]$ which is nondecreasing, equal to 0 on $(-\infty, 0]$ and equal to 1 on $[1, \infty)$. Define a function $\beta_{+}: C_{*} \rightarrow[0,1]$ as follows. The function $\beta_{+}$equals 1 on all of $C_{+T}^{\prime}$ and 0 on all of $C_{-T}^{\prime}$. On the cylinder $\Sigma_{i} \subset \Sigma^{\prime}$ for $i>0$, define $\beta_{+}:=\beta\left(\left(s-s_{i}-h r\right) /(h r)\right)$. On the rest of $\Sigma^{\prime}$, define $\beta_{+}:=0$. Similarly, define $\beta_{-}: C_{*} \rightarrow[0,1]$ to equal 1 on all of $C_{-T}^{\prime}$, to equal 0 on all of $C_{+T}^{\prime}$, to equal $\beta\left(\left(-s+s_{i}-h r\right) /(h r)\right)$ on $\Sigma_{i}$ for $i<0$ and to equal 0 on the rest of $\Sigma^{\prime}$.

The map $u_{*}$ is defined as follows. On $C_{ \pm T}^{\prime}$, the map $u_{*}$ agrees with the map $u_{ \pm T}$. On $\Sigma^{\prime}$, off of the cylinders $\Sigma_{i}$, the map $u_{*}$ agrees with the composition

$$
\Sigma \stackrel{\pi}{\longrightarrow} \mathbb{R} \times S^{1} \stackrel{\text { id } \times \alpha}{\longrightarrow} \mathbb{R} \times Y .
$$

On $\Sigma_{i}$ for $i>0$, with the notation as in (5.1), define

$$
\eta_{i, T}(s, \tau):=\eta_{i}\left(s-\left(s_{+}+T_{+}\right), \tau\right)
$$

and

$$
u_{*}(s, \tau):=\left(s, t, \beta_{+}(s, \tau) \eta_{i, T}(s, \tau)\right) .
$$

When $s \leq s_{i}+h r$, this agrees with the composition (5.4), and when $s \geq$ $s_{i}+2 h r$, this agrees with the $s \mapsto s+s_{+}+T_{+}$translate of the $i$ th negative end of $u_{+}$. Likewise, on $\Sigma_{i}$ for $i<0$, with the notation as in (5.2), define

$$
\eta_{i, T}(s, \tau):=\eta_{i}\left(s-\left(s_{-}-T_{-}\right), \tau\right)
$$

and

$$
u_{*}(s, \tau):=\left(s, t, \beta_{-}(s, \tau) \eta_{i, T}(s, \tau)\right) .
$$

When $s \geq s_{i}-h r$, this agrees with the composition (5.4), and when $s \leq$ $s_{i}-2 h r$, this agrees with the $s \mapsto s+s_{-}-T_{-}$translate of the $i$ th positive end of $u_{-}$.

5.3. Deforming the pregluing. Let $\psi_{+}$be a section of the normal bundle of $u_{+T}$, let $\psi_{-}$be a section of the normal bundle of $u_{-T}$ and let $\psi_{\Sigma}$ be a complex function on $\Sigma$. Assuming that $\psi_{ \pm}$and $\psi_{\Sigma}$ have pointwise norm less than $\delta_{0}$, we now explain how to use the data $\left(\psi_{-}, \psi_{\Sigma}, \psi_{+}\right)$to define a deformation of the map $u_{*}$. 
The coordinate $w$ on a neighborhood of $\mathbb{R} \times \alpha$ trivializes the normal bundles to the positive ends of $u_{-}$and the negative ends of $u_{+}$near $\mathbb{R} \times \alpha$. Hence the normal bundles to $C_{-T}^{\prime}$ and $C_{+T}^{\prime}$ and the trivial complex line bundle over $\Sigma^{\prime}$ fit together to define a complex line bundle $E_{*}$ over $C_{*}$. The exponential maps $e_{-}, e$ and $e_{+}$fit into a map $e_{*}$ from a small radius disc bundle in $E_{*}$ to $\mathbb{R} \times Y$ defined as follows. Over $C_{ \pm T}^{\prime}$, the map $e_{*}$ is defined on the radius $\delta_{0}$ disc bundle and agrees with the appropriate translate of $e_{ \pm}$. For $x \in \Sigma^{\prime}$, the map $e_{*}$ is defined on the radius $2 \delta_{0}$ disc bundle as follows: If $u_{*}(x)=(s, t, w)$, then $e_{*}(x, v):=(s, t, w+v)$.

Next define a function $\beta_{\Sigma}: C_{*} \rightarrow[0,1]$ as follows. The function $\beta_{\Sigma}$ is identically zero on $C_{+T}^{\prime}$ and $C_{-T}^{\prime}$. On the cylinders $\Sigma_{i}$ for $i>0$, define

$$
\beta_{\Sigma}(s, \tau):=\beta\left(\left(-s+s_{+}+T_{+}-r\right) / r\right) .
$$

This is 1 where $s \leq s_{+}+T_{+}-2 r$ and 0 where $s \geq s_{+}+T_{+}-r$. On the cylinders $\Sigma_{i}$ for $i<0$, define

$$
\beta_{\Sigma}(s, \tau):=\beta\left(\left(s-s_{-}+T_{-}-r\right) / r\right) .
$$

This is 1 where $s \geq s_{-}-T_{-}+2 r$ and 0 where $s \leq s_{-}-T_{-}+r$. On the rest of $\Sigma^{\prime}$, define $\beta_{\Sigma}:=1$.

Finally, the deformation of $u_{*}$ is defined to be the map

$$
\begin{aligned}
C_{*} & \longrightarrow \mathbb{R} \times Y, \\
x & \longmapsto e_{*}\left(x, \beta_{-} \psi_{-}+\beta_{\Sigma} \psi_{\Sigma}+\beta_{+} \psi_{+}\right) .
\end{aligned}
$$

This is well defined, because under the above identifications, $\psi_{\Sigma}$ defines a section of $E_{*}$ over the support of $\beta_{\Sigma}$, while $\psi_{ \pm}$defines a section of $E_{*}$ over the support of $\beta_{ \pm}$. If $\psi_{ \pm}$and $\psi_{\Sigma}$ are smooth, then the map (5.7) is an immersion, except possibly at the ramification points in $\Sigma$.

5.4. Equation for the deformation to be $J$-holomorphic. We now write an equation for the map (5.7) to be $J$-holomorphic, for some complex structures on $C_{*}$. This equation will have the form

$$
\beta_{-} \Theta_{-}\left(\psi_{-}, \psi_{\Sigma}\right)+\beta_{\Sigma} \Theta_{\Sigma}\left(\psi_{-}, \psi_{\Sigma}, \psi_{+}\right)+\beta_{+} \Theta_{+}\left(\psi_{\Sigma}, \psi_{+}\right)=0
$$

where $\Theta_{ \pm}$is defined on all of $u_{ \pm T}$, while $\Theta_{\Sigma}$ is defined on all of $\Sigma$.

We begin by describing the schematic form of the $\Theta$ 's. By way of preparation, let $C$ denote $C_{ \pm T}$ or $\Sigma$, and let $E$ denote, respectively, the normal bundle to $C_{ \pm T}$ or the trivial complex line bundle over $\Sigma$.

Definition 5.1. Let us call a $\left(T_{ \pm}, \Sigma\right)$-dependent function

$$
F: C^{\infty}(C ; E) \longrightarrow C^{\infty}\left(C ; E \otimes_{\mathbb{C}} T^{0,1} C\right)
$$

"type 1 quadratic" if it can be written in the form

$$
F(\psi)=P(\psi)+Q(\psi) \cdot \nabla \psi
$$


where $P$ and $Q$ are (nonlinear) bundle maps with uniformly bounded derivatives to any given order in the fiber direction, obeying $|P(\psi)|<c|\psi|^{2}$ and $|Q(\psi)|<c|\psi|$. Let us call a $\left(T_{ \pm}, \Sigma\right)$-dependent function

$$
Z: C^{\infty}(C ; E) \times C^{\infty}(C ; E) \longrightarrow C^{\infty}\left(C, E \otimes_{\mathbb{C}} T^{0,1} C\right)
$$

"type 2 quadratic" if it can be written as

$$
Z\left(\psi_{1}, \psi_{2}\right)=a\left(\psi_{1}, \psi_{2}\right)+b_{1}\left(\psi_{1}, \psi_{2}\right) \cdot \nabla \psi_{2}+b_{2}\left(\psi_{1}, \psi_{2}\right) \cdot \nabla \psi_{1},
$$

where $a, b_{1}, b_{2}$ are (nonlinear) bundle maps with uniformly bounded derivatives to any given order in the fiber direction, obeying $\left|a\left(\psi_{1}, \psi_{2}\right)\right|<c\left|\psi_{1}\right|\left|\psi_{2}\right|$, $\left|b_{1}\left(\psi_{1}, \psi_{2}\right)\right|<c\left|\psi_{1}\right|$ and $\left|b_{2}\left(\psi_{1}, \psi_{2}\right)\right|<c\left|\psi_{2}\right|$.

Now let $\psi_{ \pm}$be a section of the normal bundle of $u_{ \pm T}$ with $\left|\psi_{ \pm}\right|<\delta_{0}$. Then as in Section 2.1, the composition of $\psi_{ \pm}$with the exponential map $e_{ \pm}$ defines a $J$-holomorphic map $C_{ \pm T} \rightarrow \mathbb{R} \times Y$, for some complex structure on $C_{ \pm T}$, if and only if $D_{ \pm} \psi_{ \pm}+F_{ \pm}\left(\psi_{ \pm}\right)=0$, where $D_{ \pm}$denotes the usual linear deformation operator, while $F_{ \pm}$is type 1 quadratic. If $\psi_{\Sigma}$ is a complex function on $\Sigma$ with $\left|\psi_{\Sigma}\right|<4 \delta_{0}$, then the map $\Sigma \rightarrow \mathbb{R} \times Y$ sending $x \mapsto$ $e\left(\pi(x), \psi_{\Sigma}(x)\right)$ is $J$-holomorphic, for some complex structure on $\Sigma$, if and only if it satisfies an equation of the form $D_{\Sigma} \psi_{\Sigma}+F_{\Sigma}\left(\psi_{\Sigma}\right)=0$. Here, as in Section 2.1, the operator $D_{\Sigma}$ has the form

$$
D_{\Sigma} \psi=\bar{\partial} \psi+(\nu \psi+\mu \bar{\psi}) \otimes d \bar{z} .
$$

(For more about the operator $D_{\Sigma}$, see Section I.2.3.) Meanwhile, $F_{\Sigma}$ is type 1 quadratic, except near the ramification points in $\Sigma$ (see (5.22) below).

With the preceding understood, $\Theta_{-}$has the form

$\Theta_{-}=D_{-} \psi_{-}+F_{-}^{\prime}\left(\psi_{-}\right)+\frac{\beta_{-}}{2} \frac{\partial \beta_{\Sigma}}{\partial s}\left((d \bar{z}+\mathfrak{m}) \psi_{\Sigma}+\mathfrak{z}_{-}\left(\psi_{-}, \psi_{\Sigma}\right)\right)+\mathfrak{q}_{-} \cdot \psi_{-}+\mathfrak{q}_{-}^{\prime} \cdot \nabla \psi_{-}$.

Here $F_{-}^{\prime}$ is type 1 quadratic and differs from $F_{-}$only in the part of each cylinder $\Sigma_{i}$ for $i<0$, where $s_{i}-2 h r<s<s_{i}-h r$. Next, $\mathfrak{m}$ is a bundle map on each cylinder $\Sigma_{i}$ for $i<0$ satisfying $|\mathfrak{m}|<c\left|\eta_{-T}\right|$, where $\eta_{-T}:=\eta_{i, T}$. The function $\mathfrak{z}_{-}$is type 2 quadratic. Finally, $\mathfrak{q}_{-}$and $\mathfrak{q}_{-}^{\prime}$ are bundle maps supported in the cylinders $\Sigma_{i}$ for $i<0$ where $s_{i}-2 h r<s<s_{i}-h r$, which satisfy $\left|\mathfrak{q}_{-}\right|,\left|\mathfrak{q}_{-}^{\prime}\right|<c\left|\eta_{-T}\right|$.

Likewise, $\Theta_{+}$has the form

$\Theta_{+}=D_{+} \psi_{+}+F_{+}^{\prime}\left(\psi_{+}\right)+\frac{\beta_{+}}{2} \frac{\partial \beta_{\Sigma}}{\partial s}\left((d \bar{z}+\mathfrak{m}) \psi_{\Sigma}+\mathfrak{z}_{+}\left(\psi_{+}, \psi_{\Sigma}\right)\right)+\mathfrak{q}_{+} \cdot \psi_{+}+\mathfrak{q}_{+}^{\prime} \cdot \nabla \psi_{+} \cdot$

The terms in (5.12) satisfy the obvious analogs of the conditions on the terms in (5.11). 
Finally, $\Theta_{\Sigma}$ has the form

$$
\begin{aligned}
\Theta_{\Sigma}= & D_{\Sigma} \psi_{\Sigma}+F_{\Sigma}^{\prime}\left(\psi_{\Sigma}\right)+\mathfrak{q}_{0} \cdot \psi_{\Sigma}+\mathfrak{q}_{0}^{\prime} \cdot \nabla \psi_{\Sigma}+\mathfrak{p}_{-}\left(\eta_{-T}\right)+\beta_{-\mathfrak{z}_{0-}}\left(\psi_{-}, \psi_{\Sigma}\right) \\
& +\frac{1}{2} \frac{\partial \beta_{-}}{\partial s}\left(\left(\eta_{-T}+\psi_{-}\right) d \bar{z}+\mathfrak{z}_{0-}^{\prime}\left(\psi_{-}, \psi_{\Sigma}\right)\right)+\mathfrak{p}_{+}\left(\eta_{+T}\right)+\beta_{+} \mathfrak{z}_{0+}\left(\psi_{+}, \psi_{\Sigma}\right) \\
& +\frac{1}{2} \frac{\partial \beta_{+}}{\partial s}\left(\left(\eta_{+T}+\psi_{+}\right) d \bar{z}+\mathfrak{z}_{0+}^{\prime}\left(\psi_{+}, \psi_{\Sigma}\right)\right) .
\end{aligned}
$$

Here $F_{\Sigma}^{\prime}$ is type 1 quadratic (except near the ramification points) and differs from $F_{\Sigma}$ only in the cylinders $\Sigma_{i}$ for $i>0$ where $s_{+}+T_{+}-2 r<s<s_{+}+$ $T_{+}-r$, and in the cylinders $\Sigma_{i}$ for $i<0$ where $s_{-}-T_{-}+r<s<s_{-}-T_{-}+2 r$. The terms $\mathfrak{p}_{-}$, and $\mathfrak{p}_{+}$are type 1 quadratic; $\mathfrak{p}_{+}$is supported in the cylinders $\Sigma_{i}$ for $i>0$ where $s_{i}+h r<s<s_{i}+2 h r$; and $\mathfrak{p}_{-}$is supported in the cylinders $\Sigma_{i}$ for $i<0$ where $s_{i}-2 h r<s<s_{i}-h r$. The q's can be written as $\mathfrak{q}_{0}=\mathfrak{q}_{0-}+\mathfrak{q}_{0+}$ and $\mathfrak{q}_{0}^{\prime}=\mathfrak{q}_{0-}^{\prime}+\mathfrak{q}_{0+}^{\prime}$, where $\mathfrak{q}_{0+}$ and $\mathfrak{q}_{0+}^{\prime}$ are supported in the cylinders $\Sigma_{i}$ for $i>0$ where $s_{i}+h r<s<s_{+}+T_{+}-r+1$ and satisfy $\left|\mathfrak{q}_{0+}\right|,\left|\mathfrak{q}_{0+}^{\prime}\right|<c\left|\eta_{+T}\right|$. Likewise, $\mathfrak{q}_{0-}$ and $\mathfrak{q}_{0-}^{\prime}$ are supported in the cylinders $\Sigma_{i}$ for $i<0$ where $s_{-}-T_{-}+r-1<s<s_{i}-h r$ and satisfy $\left|\mathfrak{q}_{0_{-}}\right|,\left|\mathfrak{q}_{0_{-}}^{\prime}\right|<c\left|\eta_{-T}\right|$. The functions $\mathfrak{z}_{0 \pm}$ and $\mathfrak{z}_{0 \pm}^{\prime}$ are supported in the cylinders $\Sigma_{i}$ for $\pm i>0$, and are type 2 quadratic.

We formulate the above as a lemma:

Lemma 5.2. There exist functions $\Theta_{-}, \Theta_{+}$and $\Theta_{\Sigma}$ of the form (5.11), (5.12) and (5.13), respectively, such that the map (5.7) is J-holomorphic for some complex structure on $C_{*}$ if and only if equation (5.8) holds.

Proof. On $C_{ \pm T}^{\prime}$, equation (5.8) reads $D_{ \pm} \psi_{ \pm}+F_{ \pm}\left(\psi_{ \pm}\right)=0$; and on $\Sigma^{\prime}$, off of the cylinders $\Sigma_{i}$, equation (5.8) reads $D_{\Sigma} \psi_{\Sigma}+F_{\Sigma}\left(\psi_{\Sigma}\right)=0$. Hence, we need only consider the cylinders $\Sigma_{i}$, w.l.o.g. with $i<0$. Here, we need to show that the various terms in $\Theta_{-}$and $\Theta_{\Sigma}$ can be chosen so that

$D_{\Sigma}\left(\beta_{-}\left(\eta_{-T}+\psi_{-}\right)+\beta_{\Sigma} \psi_{\Sigma}\right)+F_{\Sigma}\left(\beta_{-}\left(\eta_{-T}+\psi_{-}\right)+\beta_{\Sigma} \psi_{\Sigma}\right)=\beta_{-} \Theta_{-}+\beta_{\Sigma} \Theta_{\Sigma}$.

To start, it follows from (5.10) that

$$
\begin{aligned}
D_{\Sigma}\left(\beta_{-}\left(\eta_{-T}+\psi_{-}\right)+\beta_{\Sigma} \psi_{\Sigma}\right)= & \beta_{-}\left(\frac{\beta_{-}}{2} \frac{\partial \beta_{\Sigma}}{\partial s} \psi_{\Sigma} d \bar{z}+D_{\Sigma}\left(\eta_{-T}+\psi_{-}\right)\right) \\
& +\beta_{\Sigma}\left(\frac{1}{2} \frac{\partial \beta_{-}}{\partial s}\left(\eta_{-T}+\psi_{-}\right) d \bar{z}+D_{\Sigma} \psi_{\Sigma}\right) .
\end{aligned}
$$

Here we have inserted some extra factors of $\beta_{-}$and $\beta_{\Sigma}$, using the fact that $\beta_{-}=1$ on the support of $\partial_{s} \beta_{\Sigma}$, and $\beta_{\Sigma}=1$ on the support of $\partial_{s} \beta_{-}$. Next,

$$
D_{-} \psi_{-}+F_{-}\left(\psi_{-}\right)=D_{\Sigma}\left(\eta_{-T}+\psi_{-}\right)+F_{\Sigma}\left(\eta_{-T}+\psi_{-}\right)
$$

because by (5.3), the two sides of (5.16) measure the failure of the same immersed surface to be $J$-holomorphic. By (5.15) and (5.16) equation (5.14) 
that we need to prove reduces to

$$
\begin{aligned}
F_{\Sigma}\left(\beta_{-}\left(\eta_{-T}+\psi_{-}\right)+\beta_{\Sigma} \psi_{\Sigma}\right)-\beta_{-} F_{\Sigma}\left(\eta_{-T}+\psi_{-}\right)-\beta_{\Sigma} F_{\Sigma}\left(\psi_{\Sigma}\right) \\
=\beta_{-}\left(F_{-}^{\prime \prime}\left(\psi_{-}\right)+\frac{\beta_{-}}{2} \frac{\partial \beta_{\Sigma}}{\partial s}\left(\mathfrak{m} \psi_{\Sigma}+\mathfrak{z}_{-}\left(\psi_{-}, \psi_{\Sigma}\right)\right)+\mathfrak{q}_{-} \cdot \psi_{-}+\mathfrak{q}_{-}^{\prime} \cdot \nabla \psi_{-}\right) \\
\quad+\beta_{\Sigma}\left(F_{\Sigma}^{\prime \prime}\left(\psi_{\Sigma}\right)+\mathfrak{q}_{0} \cdot \psi_{\Sigma}+\mathfrak{q}_{0}^{\prime} \cdot \nabla \psi_{\Sigma}+\mathfrak{p}_{-}\left(\eta_{-T}\right)+\beta_{-\mathfrak{z}_{0}-}\left(\psi_{-}, \psi_{\Sigma}\right)\right. \\
\left.\quad+\frac{1}{2} \frac{\partial \beta_{-}}{\partial s} \mathfrak{z}_{0-}^{\prime}\left(\psi_{-}, \psi_{\Sigma}\right)\right) .
\end{aligned}
$$

Here $F_{-}^{\prime \prime}:=F_{-}^{\prime}-F_{-}$and $F_{\Sigma}^{\prime \prime}:=F_{\Sigma}^{\prime}-F_{\Sigma}$ are type 1 quadratic, and supported in our cylinder $\Sigma_{i}$ where $s_{i}-2 h r<s<s_{i}-h r$ and $s_{-}-T_{-}+r<s<$ $s_{-}-T_{-}+2 r$, respectively.

To prepare for the proof of $(5.17)$, first note that since $F:=F_{\Sigma}$ is type 1 quadratic, it follows that

$$
F\left(\psi_{1}+\psi_{2}\right)=F\left(\psi_{1}\right)+F\left(\psi_{2}\right)+F_{1}\left(\psi_{1}, \psi_{2}\right),
$$

where $F_{1}$ is type 2 quadratic. This last condition implies that

$$
F_{1}\left(\psi_{1}+\psi_{2}, \psi_{3}\right)=F_{1}\left(\psi_{1}, \psi_{3}\right)+F_{1}\left(\psi_{2}, \psi_{3}\right)+F_{2}\left(\psi_{1}, \psi_{2}, \psi_{3}\right),
$$

where $F_{2}$ has the form

$$
F_{2}\left(\psi_{1}, \psi_{2}, \psi_{3}\right)=a\left(\psi_{1}, \psi_{2}, \psi_{3}\right)+\sum_{i=1}^{3} b_{i}\left(\psi_{1}, \psi_{2}, \psi_{3}\right) \cdot \nabla \psi_{i}
$$

with $\left|a\left(\psi_{1}, \psi_{2}, \psi_{3}\right)\right|<c\left|\psi_{1}\right|\left|\psi_{2}\right|\left|\psi_{3}\right|, b_{1}\left(\psi_{1}, \psi_{2}, \psi_{3}\right)|<c| \psi_{2}|| \psi_{3} \mid$, and so forth.

To prove (5.17), use (5.18) and (5.19) to expand the left hand side as

$$
\begin{aligned}
& F\left(\beta_{-}\left(\eta_{-T}+\psi_{-}\right)+\beta_{\Sigma} \psi_{\Sigma}\right)-\beta_{-} F\left(\eta_{-T}+\psi_{-}\right)-\beta_{\Sigma} F\left(\psi_{\Sigma}\right) \\
& =F_{1}\left(\beta_{-} \eta_{-T}, \beta_{\Sigma} \psi_{\Sigma}\right) \\
& +F_{1}\left(\beta_{-} \psi_{-}, \beta_{\Sigma} \psi_{\Sigma}\right)+F_{2}\left(\beta_{-} \eta_{-T}, \beta_{-} \psi_{-}, \beta_{\Sigma} \psi_{\Sigma}\right) \\
& +\left(F_{1}\left(\beta_{-} \eta_{-T}, \beta_{-} \psi_{-}\right)-\beta_{-} F_{1}\left(\eta_{-T}, \psi_{-}\right)\right) \\
& +\left(F\left(\beta_{-} \eta_{-T}\right)-\beta_{-} F\left(\eta_{-T}\right)\right) \\
& \quad+\left(F\left(\beta_{-} \psi_{-}\right)-\beta_{-} F\left(\psi_{-}\right)\right) \\
& +\left(F\left(\beta_{\Sigma} \psi_{\Sigma}\right)-\beta_{\Sigma} F\left(\psi_{\Sigma}\right)\right) .
\end{aligned}
$$

On the right side of (5.20), the first line gives the $\mathfrak{m}, \mathfrak{q}_{0}$ and $\mathfrak{q}_{0}^{\prime}$ terms on the right side of (5.17). The second line gives the $\mathfrak{z}_{-}, \mathfrak{z}_{0}$ and $\mathfrak{z}_{0}^{\prime}$ terms. The third line gives the $\mathfrak{q}_{-}$and $\mathfrak{q}_{-}^{\prime}$ terms. The fourth line gives the $\mathfrak{p}_{-}$term, the fifth line gives the $F_{-}^{\prime \prime}$ term and the last line gives the $F_{\Sigma}^{\prime \prime}$ term. Here we have used the fact that $\left|\nabla \eta_{-T}\right|<c\left|\eta_{-T}\right|$, which follows from the results in Section 2 . Also, we are assuming that $r>r h>1$, so that $\left|\partial_{s} \beta_{-}\right|,\left|\partial_{s} \beta_{\Sigma}\right|<c$. 
To complete the picture of equation (5.8), we now describe the behavior of the term $F_{\Sigma}^{\prime}\left(\psi_{\Sigma}\right)$ in (5.13) near a ramification point of the branched cover $\pi: \Sigma \rightarrow \mathbb{R} \times S^{1}$. Recall that $F_{\Sigma}^{\prime}\left(\psi_{\Sigma}\right)=F_{\Sigma}\left(\psi_{\Sigma}\right)$ near the ramification points. Also recall our coordinates $(z=s+i t, w)$ on a neighborhood of $\mathbb{R} \times \alpha$ in $\mathbb{R} \times Y$. On the complement of the ramification points, equations $(2.2)$ and (2.3) imply that

$$
F_{\Sigma}\left(\psi_{\Sigma}\right)=a\left(t, \psi_{\Sigma}\right) \frac{\partial \psi_{\Sigma}}{\partial z} d \bar{z}+P\left(t, \psi_{\Sigma}\right)
$$

where $a(t, w)$ and $P(t, w)$ are smooth functions of their arguments which vanish where $w=0$. Near a ramification point, choose a local holomorphic coordinate $u$ on $\Sigma$ such that $\pi^{*} z=z_{0}+u^{q+1}$, with $q$ a positive integer. It follows from (5.21) that near the ramification point,

$$
F_{\Sigma}^{\prime}\left(\psi_{\Sigma}\right)=F_{\Sigma}\left(\psi_{\Sigma}\right)=\left(\frac{\bar{u}}{u}\right)^{q} a\left(t, \psi_{\Sigma}\right) \frac{\partial \psi_{\Sigma}}{\partial u} d \bar{u}+P\left(t, \psi_{\Sigma}\right)
$$

In particular, $F_{\Sigma}^{\prime}\left(\psi_{\Sigma}\right)$ is generally not continuous at the ramification points, even when $\psi_{\Sigma}$ is smooth.

5.5. Banach space setup. We now select appropriate Banach spaces to use in solving equation (5.8).

Let $C$ denote $C_{ \pm}$or $\Sigma$, and let $E$ denote, respectively, the normal bundle to $C_{ \pm}$or the trivial complex line bundle on $\Sigma$. Let $\mathcal{H}_{0}(C)$ denote the Banach space obtained by completing the space of compactly supported sections of $E \otimes T^{0,1} C$ using the norm $\|\cdot\|$ defined by

$$
\|\eta\|:=\left(\int_{C}|\eta|^{2}\right)^{1 / 2}+\left(\sup _{x \in C} \sup _{\rho \in(0,1]} \rho^{-1 / 2} \int_{\operatorname{dist}(x, \cdot)<\rho}|\eta|^{2}\right)^{1 / 2} .
$$

Here we have chosen a metric on each $\Sigma \in \mathcal{M}$ as in Section I.2.3, and we use the metric on $C_{ \pm}$induced by its immersion $u_{ \pm}$into $\mathbb{R} \times Y$. (Note that for our purposes, the exponent of $\rho$ above could be replaced by $-v$ for any $v \in(0,1)$. We will fix $v=1 / 2$ for definiteness.)

Next, let $\mathcal{H}_{1}(C)$ denote the completion of the space of compactly supported sections of $E$ using the norm $\|\cdot\|_{*}$ defined by

$$
\|\eta\|_{*}:=\|\nabla \eta\|+\|\eta\| \text {. }
$$

Lemma 5.3. The tautological map $\psi \mapsto \psi$ defines a bounded map from $\mathcal{H}_{1}(C)$ into the Banach space of sections of $E$ that are Holder continuous with exponent $1 / 4$, and decay to zero on the ends of $C$.

Proof. This is a consequence of [12, Thm 3.5.2], together with the fact that $C$ has bounded geometry.

Now let $D_{C}: C^{\infty}(E) \rightarrow C^{\infty}\left(E \otimes T^{0,1} C\right)$ denote the deformation operator $D_{ \pm}$when $C=C_{ \pm}$, or the operator $D_{\Sigma}$ when $C=\Sigma$. This extends as a 
bounded operator from $L_{1}^{2}(E)$ to $L^{2}\left(E \otimes T^{0,1} C\right)$, and also as a bounded operator from $\mathcal{H}_{1}(C)$ to $\mathcal{H}_{0}(C)$.

Lemma 5.4. There is a positive constant $\gamma_{C}$ such that

$$
\left\|D_{C} \eta\right\| \geq \gamma_{C}\|\eta\|_{*}
$$

for all $\eta$ in $\mathcal{H}_{1}(C)$ that are $L^{2}$-orthogonal to the kernel of $D_{C}$.

Proof. Our assumption that all Reeb orbits are nondegenerate guarantees that the operator $D_{C}$ is Fredholm. Since $D_{C}$ has closed range, there exists $\gamma>0$ such that $\left\|D_{C} \eta\right\|_{2} \geq \gamma\|\eta\|_{L_{1}^{2}}$ whenever $\eta \in L_{1}^{2}$ is orthogonal to the kernel of $D_{C}$. The lemma follows from this and [12, Thm 5.4.1].

Recall from Lemma I.2.15(b) that the operator $D_{\Sigma}$ has trivial kernel. Lemma 5.4 then finds a positive constant $\gamma_{\Sigma}$ for each branched cover $\Sigma \in \mathcal{M}$, such that $\left\|D_{\Sigma} \eta\right\| \geq \gamma_{\Sigma}\|\eta\|_{*}$ for all $\eta \in \mathcal{H}_{1}(\Sigma)$. We will need a positive lower bound on $\left\{\gamma_{\Sigma}\right\}$ as $\Sigma$ varies over all of $\mathcal{M}$, where the multiplicities $\left(a_{1}, \ldots, a_{N_{+}} \mid a_{-1}, \ldots, a_{-N_{-}}\right)$entering into the definition of $\mathcal{M}$ are fixed.

Lemma 5.5. There exists a $\Sigma$-independent constant $\gamma>0$ such that for any $\Sigma \in \mathcal{M}$ and $\eta \in \mathcal{H}_{1}(\Sigma)$, we have $\left\|D_{\Sigma} \eta\right\| \geq \gamma\|\eta\|_{*}$.

Proof. First observe that it is enough to find $\gamma>0$ with $\left\|D_{\Sigma} \eta\right\|_{2} \geq \gamma\|\eta\|_{L_{1}^{2}}$ for all $\Sigma \in \mathcal{M}$ and $\eta \in L_{1}^{2}\left(\pi^{*} N\right)$. This follows from the proof of $[\mathbf{1 2}$, Thm 5.4.1], because that argument uses only the local geometry in discs of radius 1 , and the local geometry is uniformly controlled over all branched covers.

Now suppose that there does not exist $\gamma>0$ such that $\left\|D_{\Sigma} \eta\right\|_{2} \geq \gamma\|\eta\|_{L_{1}^{2}}$ for all $\Sigma$ and $\eta$. Then we can find a sequence of branched covers $\left\{\Sigma_{k}\right\}_{k=1,2, \ldots \text {, }}$ and for each $\Sigma_{k}$ an element $\eta_{k}$ of the domain of the corresponding operator $D_{\Sigma_{k}}$, such that $\left\|D_{\Sigma_{k}} \eta_{k}\right\|_{2} \leq 1 / k$ and $\left\|\eta_{k}\right\|_{2}+\left\|\nabla \eta_{k}\right\|_{2}=1$. For any $\Sigma$, since $D_{\Sigma}$ is a first-order elliptic operator, there is a constant $b$ such that $\|\nabla \eta\|_{2} \leq b\left(\left\|D_{\Sigma} \eta\right\|_{2}+\|\eta\|_{2}\right)$ for all $\eta \in L_{1}^{2}\left(\pi^{*} N\right)$. The constant $b$ can be chosen independently of $\Sigma$ because it depends only on the local geometry of the branched cover. Thanks to the existence of $b$, we can choose a new sequence $\left\{\left(\Sigma_{k}, \eta_{k}\right)\right\}$ such that $\left\|D_{\Sigma_{k}} \eta_{k}\right\|_{2} \leq 1 / k$ and $\left\|\eta_{k}\right\|_{2}=1$.

By Lemma I.2.28, we can pass to a subsequence so that the sequence of branched covers $\left\{\Sigma_{k}\right\}$ converges in $\mathcal{M} / \mathbb{R}$, in the sense of Definition I.2.27, to a tree $T$ together with a branched cover $\Sigma_{* j}$ for each internal vertex $j$ of $T$. By a standard compactness argument using a priori elliptic estimates, we can pass to a further subsequence so that for each $j$, the sequence $\eta_{k}$, after suitable translations of the $s$ coordinate, converges to a function $\eta_{* j}$ on $\Sigma_{* j}$. The function $\eta_{* j}$ is in the kernel of $D_{\Sigma_{* j}}$, but we know that the latter operator has trivial kernel, so $\eta_{* j}=0$ for each $j$. We conclude that when $k$ is large, all but a small amount of the $L^{2}$ norm of $\eta_{k}$ comes from subcylinders in $\Sigma_{k}$ that project to long cylinders in $\mathbb{R} \times S^{1}$ and that are far away from any ramification points. 
To get a contradiction from this, note that if $\lambda$ is compactly supported on a cylinder in any $\Sigma \in \mathcal{M}$, which projects to a cylinder of the form $(a, b) \times S^{1}$ in $\mathbb{R} \times S^{1}$, then there is a constant $\gamma^{\prime}>0$, depending only on $\mathcal{M}$, such that $\left\|D_{\Sigma} \lambda\right\| \geq \gamma^{\prime}\left(\|\nabla \lambda\|_{2}+\|\lambda\|_{2}\right)$. This follows by expanding $\lambda$ in terms of eigenfunctions of the operator $L_{m}$, where $m$ denotes the covering multiplicity of the cylinder. Granted this bound, multiplication of $\eta_{k}$ by suitable cutoff functions shows that long tubes as above cannot account for most of its $L^{2}$ norm.

5.6. Solving for $\psi_{-}$and $\psi_{+}$in terms of $\psi_{\Sigma}$. Our strategy for solving equation (5.8) is to solve the three equations

$$
\begin{aligned}
\Theta_{-}\left(\psi_{-}, \psi_{\Sigma}\right)=0 & \text { on all of } u_{-T}, \\
\Theta_{+}\left(\psi_{\Sigma}, \psi_{+}\right)=0 & \text { on all of } u_{+T}, \\
\Theta_{\Sigma}\left(\psi_{-}, \psi_{\Sigma}, \psi_{+}\right)=0 & \text { on all of } \Sigma .
\end{aligned}
$$

More precisely, let $\mathcal{H}_{\Sigma}:=\mathcal{H}_{1}(\Sigma)$, and let $\mathcal{H}_{ \pm}$denote the orthogonal complement of $\operatorname{Ker}\left(D_{ \pm}\right)$in $\mathcal{H}_{1}\left(u_{ \pm T}\right)$; we will solve the above equations for $\psi_{ \pm} \in \mathcal{H}_{ \pm}$ and $\psi_{\Sigma} \in \mathcal{H}_{\Sigma}$.

Given $\psi_{\Sigma}$, we now explain how to solve equations (5.24) and (5.25) for $\psi_{-}$and $\psi_{+}$, respectively. Later, we will plug the results into (5.26) in order to view (5.26) as an equation for $\psi_{\Sigma}$ alone.

To prepare for subsequent estimates, for $i=1, \ldots, \bar{N}_{+}$define $\lambda_{i}$ to be the largest negative eigenvalue of the asymptotic operator $L_{a_{i}}$, and define $\lambda_{+}:=\min \left\{\left|\lambda_{i}\right|\right\}_{i=1, \ldots, \bar{N}_{+}}$. Likewise, for $i=-1, \ldots,-\bar{N}_{-}$, let $\lambda_{i}$ denote the smallest positive eigenvalue of $L_{a_{i}}$, and define $\lambda_{-}:=\min \left\{\lambda_{i}\right\}_{i=-1, \ldots,-\bar{N}_{-}}$.

Proposition 5.6. Fix $h \in(0,1)$. There exist constants $r_{0}>h^{-1}$ and $\varepsilon, c>0$ such that the following holds: Fix $r>r_{0}$ and $T_{-}, T_{+} \geq 5 r$. Fix $\Sigma \in \mathcal{M}$, and let $\mathcal{B}_{\Sigma}$ denote the ball of radius $\varepsilon$ in $\mathcal{H}_{\Sigma}$. Then:

(a) There exist maps $\psi_{-}$and $\psi_{+}$, from $\mathcal{B}_{\Sigma}$ into the radius $\varepsilon$ balls in $\mathcal{H}_{-}$and $\mathcal{H}_{+}$, respectively, such that $\psi_{-}=\psi_{-}\left(\psi_{\Sigma}\right)$ solves (5.24) and $\psi_{+}=\psi_{+}\left(\psi_{\Sigma}\right)$ solves (5.25).

(b) $\psi_{ \pm}$, when identified with a section of the normal bundle to the untranslated curve $u_{ \pm}$, varies smoothly as $\left(T_{-}, T_{+}\right)$and $\psi_{\Sigma}$ are varied.

(c) $\left\|\psi_{ \pm}\left(\psi_{\Sigma}\right)\right\|_{*} \leq c r^{-1}\left\|\psi_{\Sigma}\right\|_{*}$.

(d) The derivative of $\psi_{ \pm}$at a point $\psi_{\Sigma} \in \mathcal{B}_{\Sigma}$ defines a bounded linear functional $\mathcal{D}: \mathcal{H}_{\Sigma} \rightarrow \mathcal{H}_{ \pm}$obeying

$$
\|\mathcal{D} \eta\|_{*} \leq c r^{-1}\|\eta\|_{*} .
$$

Proof. (a) We will explain how to solve equation (5.24) for $\psi_{-}$; an analogous procedure solves equation (5.25) for $\psi_{+}$. To start, use (5.11) to rewrite $(5.24)$ as

$$
D_{-} \psi_{-}+\mathcal{F}_{0}\left(\psi_{\Sigma}\right)+\mathcal{F}_{1}\left(\psi_{-}, \psi_{\Sigma}\right)=0
$$


where

$$
\begin{aligned}
\mathcal{F}_{0}\left(\psi_{\Sigma}\right) & :=\frac{\beta_{-}}{2} \frac{\partial \beta_{\Sigma}}{\partial s}(d \bar{z}+\mathfrak{m}) \psi_{\Sigma} \\
\mathcal{F}_{1}\left(\psi_{-}, \psi_{\Sigma}\right) & :=F_{-}^{\prime}\left(\psi_{-}\right)+\frac{\beta_{-}}{2} \frac{\partial \beta_{\Sigma}}{\partial s} \mathfrak{z}_{-}\left(\psi_{-}, \psi_{\Sigma}\right)+\mathfrak{q}_{-} \cdot \psi_{-}+\mathfrak{q}_{-}^{\prime} \cdot \nabla \psi_{-} .
\end{aligned}
$$

By virtue of Lemma 5.3, there exists $\varepsilon>0$ such that if $\psi_{\Sigma} \in \mathcal{H}_{\Sigma}$ satisfies $\left\|\psi_{\Sigma}\right\|_{*}<\varepsilon$, then $\left|\psi_{\Sigma}\right|<\delta_{0}$; and if $\psi_{-} \in \mathcal{H}_{1}\left(u_{-T}\right)$ satisfies $\left\|\psi_{-}\right\|_{*}<\varepsilon$, then $\left|\psi_{-}\right|<\delta_{0}$. So if $\left\|\psi_{\Sigma}\right\|_{*}<\varepsilon$, then $\mathcal{F}_{0}\left(\psi_{\Sigma}\right) \in \mathcal{H}_{0}\left(u_{-T}\right)$ is defined, and $\mathcal{F}_{1}\left(\cdot, \psi_{\Sigma}\right)$ defines a smooth map from the radius $\varepsilon$ ball in $\mathcal{H}_{1}\left(u_{-T}\right)$ to $\mathcal{H}_{0}\left(u_{-T}\right)$.

To solve (5.27), we will apply the contraction mapping theorem to a map $\mathcal{I}$ defined as follows. Lemma 5.4 implies that $D_{-}$has a bounded inverse $D_{-}^{-1}: \mathcal{H}_{0}\left(u_{-T}\right) \rightarrow \mathcal{H}_{-}$. Consequently, for fixed $\psi_{\Sigma}$ with $\left\|\psi_{\Sigma}\right\|_{*}<\varepsilon$, the assignment

$$
\psi_{-} \longmapsto \mathcal{I}\left(\psi_{-}\right):=-D_{-}^{-1}\left(\mathcal{F}_{0}\left(\psi_{\Sigma}\right)+\mathcal{F}_{1}\left(\psi_{-}, \psi_{\Sigma}\right)\right)
$$

defines a smooth map from the radius $\varepsilon$ ball in $\mathcal{H}_{-}$to $\mathcal{H}_{-}$.

Claim. If $r$ and $T_{-}$are sufficiently large and $\varepsilon>0$ is sufficiently small, then the map $\mathcal{I}$ sends the radius $\varepsilon$ ball in $\mathcal{H}_{-}$to itself as a contraction mapping with

$$
\left\|\mathcal{I}\left(\psi_{-}\right)-\mathcal{I}\left(\psi_{-}^{\prime}\right)\right\|_{*} \leq \frac{1}{2}\left\|\psi_{-}-\psi_{-}^{\prime}\right\|_{*} .
$$

Proof of claim. It follows from the definition of $\beta_{\Sigma}$ that $\left|\partial_{s} \beta_{\Sigma}\right|<c r^{-1}$, and so by (5.28) we have

$$
\left\|\mathcal{F}_{0}\left(\psi_{\Sigma}\right)\right\| \leq c r^{-1}\left\|\psi_{\Sigma}\right\|_{*}
$$

By Lemma 5.3 and the fact that $F_{-}^{\prime}$ is type 1 quadratic, we have

$$
\left\|F_{-}^{\prime}\left(\psi_{-}\right)\right\| \leq c\left\|\psi_{-}\right\|_{*}^{2} .
$$

By Lemma 5.3 and the fact that $\mathfrak{z}_{-}$is type 2 quadratic, we have

$$
\left\|\mathfrak{z}_{-}\left(\psi_{-}, \psi_{\Sigma}\right)\right\| \leq c\left\|\psi_{-}\right\|\left\|_{*}\right\| \psi_{\Sigma} \|_{*} .
$$

By the decay estimates on the ends of $u_{-}$from Section 2, we have

$$
\begin{aligned}
\left\|\mathfrak{q}_{-} \cdot \psi_{-}+\mathfrak{q}_{-}^{\prime} \cdot \nabla \psi_{-}\right\| & \leq c \sum_{i=-1}^{-\bar{N}_{-}} \exp \left(-\lambda_{i}\left(s_{i}-s_{-}+T_{-}-2 r h\right)\right)\left\|\psi_{-}\right\|_{*} \\
& \leq c \exp \left(-\lambda_{-} T_{-} / 2\right)\left\|\psi_{-}\right\|_{*} .
\end{aligned}
$$

Since $D_{-}^{-1}$ is a bounded operator, the above estimates imply that

$$
\left\|\mathcal{I}\left(\psi_{-}\right)\right\|_{*} \leq c\left(\left\|\psi_{-}\right\|_{*}^{2}+r^{-1}\left(1+\left\|\psi_{-}\right\|_{*}\right)\left\|\psi_{\Sigma}\right\|_{*}+\exp \left(-\lambda_{-} T_{-} / 2\right)\left\|\psi_{-}\right\|_{*} \cdot\right) .
$$

If $\varepsilon$ is sufficiently small and if $r$ and $T_{-}$are sufficiently large, then the right hand side of (5.31) is less than $\varepsilon$ whenever $\left\|\psi_{-}\right\|\left\|_{*}\right\| \psi_{\Sigma} \|_{*}<\varepsilon$. 
We now prove the contraction property. Since $F_{-}^{\prime}$ is type 1 quadratic,

$$
\left\|F_{-}^{\prime}\left(\psi_{-}\right)-F_{-}^{\prime}\left(\psi_{-}^{\prime}\right)\right\| \leq c\left(\left\|\psi_{-}\right\|_{*}+\left\|\psi_{-}\right\|_{*}^{2}\right)\left\|\psi_{-}-\psi_{-}^{\prime}\right\|_{*} .
$$

Since $\mathfrak{z}_{-}$is type 2 quadratic,

$$
\left\|\mathfrak{z}_{-}\left(\psi_{-}, \psi_{\Sigma}\right)-\mathfrak{z}_{-}\left(\psi_{-}^{\prime}, \psi_{\Sigma}\right)\right\| \leq c\left(\left\|\psi_{\Sigma}\right\|_{*}+\left\|\psi_{-}\right\|_{*}\left\|\psi_{\Sigma}\right\|_{*}\right)\left\|\psi_{-}-\psi_{-}^{\prime}\right\|_{*} .
$$

Thus, for $\psi_{-} \neq \psi_{-}^{\prime}$,

$$
\begin{aligned}
\frac{\left\|\mathcal{I}\left(\psi_{-}\right)-\mathcal{I}\left(\psi_{-}^{\prime}\right)\right\|}{\left\|\psi_{-}-\psi_{-}^{\prime}\right\|_{*}} \leq & c\left(\left\|\psi_{-}\right\|_{*}+\left\|\psi_{-}\right\|_{*}^{2}+r^{-1}\left\|\psi_{\Sigma}\right\|_{*}\left(1+\left\|\psi_{-}\right\|_{*}\right)\right. \\
& \left.+\exp \left(-\lambda_{-} T_{-} / 2\right)\right) .
\end{aligned}
$$

If $\varepsilon$ is sufficiently small and if $r$ and $T_{-}$are sufficiently large, then the right hand side of (5.33) is less than $\frac{1}{2}$ whenever $\left\|\psi_{-}\right\|_{*},\left\|\psi_{\Sigma}\right\|_{*}<\varepsilon$.

This completes the proof of the claim. Part (a) of the proposition now follows from the contraction mapping theorem. (b) Smoothness of the map $\psi_{-}$follows from smoothness of the maps $\mathcal{F}_{0}$ and $\mathcal{F}_{1}$ used to define the contraction mapping (5.29).

(c) By the estimate (5.31), a fixed point $\psi_{-}$of (5.29) satisfies

$$
\left\|\psi_{-}\right\|_{*} \leq c r^{-1}\left\|\psi_{\Sigma}\right\|_{*}+c\left\|\psi_{-}\right\|_{*}\left(\left\|\psi_{-}\right\|_{*}+r^{-1}\left\|\psi_{\Sigma}\right\|_{*}+\exp \left(-\lambda_{-} T_{-} / 2\right)\right) .
$$

Recall that $\left\|\psi_{\Sigma}\right\|_{*},\left\|\psi_{-}\right\|_{*}<\varepsilon$. So if $\varepsilon$ is sufficiently small and if $r$ and $T_{-}$ are sufficiently large, then the sum in parentheses on the right hand side of (5.34) is less than $c^{-1} / 2$.

(d) Regard the right hand side of (5.29) as a function of both $\psi_{-}$and $\psi_{\Sigma}$, and let $\mathcal{D}_{-}$and $\mathcal{D}_{\Sigma}$ denote its derivatives with respect to $\psi_{-}$and $\psi_{\Sigma}$. Then the derivative of $\psi_{-}$as a function of $\psi_{\Sigma}$ is given by

$$
\mathcal{D}=\left(1-\mathcal{D}_{-}\right)^{-1} \mathcal{D}_{\Sigma}
$$

By (5.30), if $\varepsilon$ is sufficiently small and if $r$ and $T_{-}$are sufficiently large, then the operator $\mathcal{D}_{-}$has norm less than $1 / 2$. On the other hand, by the analog of (5.32) in which the roles of $\psi_{-}$and $\psi_{\Sigma}$ are switched, we have

$$
\left\|\mathcal{D}_{\Sigma} \eta\right\|_{*} \leq c r^{-1}\left(1+\left\|\psi_{-}\right\|_{*}+\left\|\psi_{\Sigma}\right\|_{*}\left\|\psi_{-}\right\|_{*}\right)\|\eta\|_{*} \text {. }
$$

Putting these estimates into (5.35) completes the proof.

5.7. Solving for $\psi_{\Sigma}$. Let $h, r, T_{-}, T_{+}$be as in Proposition 5.6. Fix $\Sigma \in \mathcal{M}$. We now solve equation (5.26) for $\psi_{\Sigma} \in \mathcal{B}_{\Sigma}$. To start, write equation (5.26) as

$$
D_{\Sigma} \psi_{\Sigma}+\mathcal{F}_{\Sigma}\left(\psi_{\Sigma}\right)=0
$$

where $\mathcal{F}_{\Sigma}\left(\psi_{\Sigma}\right)$ denotes the sum of the terms other than $D_{\Sigma} \psi_{\Sigma}$ on the right hand side of (5.13). Here Proposition 5.6 is used to view $\psi_{-}$and $\psi_{+}$as functions of $\psi_{\Sigma}$. 
Equation (5.36) cannot be treated in the same way as equation (5.27), because the operator $D_{\Sigma}$ has a nontrivial cokernel. To deal with this issue, introduce the $L^{2}$-orthogonal projection $\Pi$ from $L^{2}\left(\pi^{*} N \otimes T^{0,1} \Sigma\right)$ onto $\operatorname{Ker}\left(D_{\Sigma}^{*}\right)$. Equation (5.36) is then equivalent to the two equations

$$
\begin{gathered}
D_{\Sigma} \psi_{\Sigma}+(1-\Pi) \mathcal{F}_{\Sigma}\left(\psi_{\Sigma}\right)=0 \\
\Pi \mathcal{F}_{\Sigma}\left(\psi_{\Sigma}\right)=0 .
\end{gathered}
$$

We now solve the first of these two equations.

Proposition 5.7. Fix $h \in(0,1)$. There exist constants $r_{0}>h^{-1}$ and $\varepsilon>0$ such that when $r>r_{0}$ and $T_{+}, T_{-} \geq 5 r$, the following is true. Fix $\Sigma \in \mathcal{M}$, and let $\mathcal{B}_{\Sigma}$ denote the ball of radius $\varepsilon$ in $\mathcal{H}_{\Sigma}$. Then:

(a) There exists a unique $\psi_{\Sigma} \in \mathcal{B}_{\Sigma}$ satisfying equation (5.37).

(b) This $\psi_{\Sigma}$ satisfies

$$
\begin{aligned}
\left\|\psi_{\Sigma}\right\|_{*}<c & \left(\sum_{i=1}^{\bar{N}_{+}} \exp \left(-\left|\lambda_{i}\right|\left(s_{+}-s_{i}+T_{+}-2 r h\right)\right)\right. \\
& \left.+\sum_{i=-1}^{-\bar{N}_{-}} \exp \left(-\lambda_{i}\left(s_{i}-s_{-}+T_{-}-2 r h\right)\right)\right) \\
< & c\left(\exp \left(-\lambda_{-} T_{-} / 2\right)+\exp \left(-\lambda_{+} T_{+} / 2\right)\right) .
\end{aligned}
$$

(c) This $\psi_{\Sigma}$ defines a Lipschitz section of $\pi^{*} N$ which is smooth except possibly at the ramification points of $\pi$.

Proof. To prove part (a), we apply the contraction mapping theorem to the $\operatorname{map} \mathcal{I}: \mathcal{B}_{\Sigma} \rightarrow \mathcal{H}_{\Sigma}$ defined as follows. Recall that the kernel of $D_{\Sigma}$ is trivial. Thus it makes sense to define

$$
\mathcal{I}\left(\psi_{\Sigma}\right):=-D_{\Sigma}^{-1}(1-\Pi) \mathcal{F}_{\Sigma}\left(\psi_{\Sigma}\right)
$$

under the assumptions of Proposition 5.6.

To get estimates on $\mathcal{I}$, first recall from Lemma 5.5 that there is a $\Sigma$ independent upper bound on the operator norm of $D_{\Sigma}^{-1}$. Next, given $\psi_{\Sigma} \in$ $\mathcal{B}_{\Sigma}$, we claim that on the $s \geq s_{-}-T_{-}+2 r$ part of the positive ends of $u_{-T}$, the corresponding section $\psi_{-}$satisfies

$$
\left|\psi_{-}\right|+\left|\nabla \psi_{-}\right| \leq c_{-} r^{-1}\left\|\psi_{\Sigma}\right\|_{*} \exp \left(-\lambda_{-}\left(s-\left(s_{-}-T_{-}+2 r\right)\right)\right),
$$

where $c_{-}$depends only on $u_{-}$. This follows from decay estimates as in Lemma 2.3, together with Proposition 5.6(c). Likewise, there is a constant $c_{+}$, depending only on $u_{+}$, such that on the $s \leq s_{+}+T_{+}-2 r$ part of the negative ends of $u_{+T}$, we have

$$
\left|\psi_{+}\right|+\left|\nabla \psi_{+}\right| \leq c_{+} r^{-1}\left\|\psi_{\Sigma}\right\|_{*} \exp \left(-\lambda_{+}\left(\left(s_{+}+T_{+}-2 r\right)-s\right)\right) .
$$


Estimating the individual terms in $\mathcal{I}$ as in the proof of Proposition 5.6, and using Proposition 5.6(c) together with (5.40) and (5.41), we find that

$$
\begin{aligned}
\left\|\mathcal{I}\left(\psi_{\Sigma}\right)\right\|_{*} \leq & c\left(\left\|\psi_{\Sigma}\right\|_{*}^{2}+e^{-\lambda r}\left\|\psi_{\Sigma}\right\|_{*}+\sum_{i=1}^{\bar{N}_{+}} e^{-\left|\lambda_{i}\right|\left(s_{+}-s_{i}+T_{+}-2 r h\right)}\right. \\
& \left.+\sum_{i=-1}^{-\bar{N}_{-}} e^{-\lambda_{i}\left(s_{i}-s_{-}+T_{-}-2 r h\right)}\right) \\
\leq & c\left(\left\|\psi_{\Sigma}\right\|_{*}^{2}+e^{-\lambda r}\left\|\psi_{\Sigma}\right\|_{*}+e^{-\lambda_{-} T_{-} / 2}+e^{-\lambda_{+} T_{+} / 2}\right)
\end{aligned}
$$

where $\lambda:=\min \left\{\lambda_{-}, \lambda_{+}\right\}$.

It follows from (5.42) that if $\varepsilon$ is sufficiently small, if $r$ is sufficiently large and if $T_{-}$and $T_{+}$are sufficiently large with respect to $\varepsilon$, then $\mathcal{I}$ maps $\mathcal{B}_{\Sigma}$ to itself. Now if $\psi_{\Sigma}$ and $\psi_{\Sigma}^{\prime}$ are distinct elements of $\mathcal{B}_{\Sigma}$, then using Proposition 5.6(c),(d), assuming that $\varepsilon<1$, we find that there is a constant $c$ with

$$
\frac{\left\|\mathcal{I}\left(\psi_{\Sigma}\right)-\mathcal{I}\left(\psi_{\Sigma}^{\prime}\right)\right\|_{*}}{\left\|\psi_{\Sigma}-\psi_{\Sigma}^{\prime}\right\|_{*}} \leq c\left(\left\|\psi_{\Sigma}\right\|_{*}+r^{-1}\right) .
$$

So $\mathcal{I}$ is a contraction mapping on $B_{\Sigma}$ provided that $\varepsilon$ is sufficiently small and $r$ is sufficiently large. Then $\mathcal{I}$ has a unique fixed point in $B_{\Sigma}$, which by definition satisfies (5.37).

Part (b) follows from (5.42) provided that $\varepsilon$ is sufficiently small and $r$ is sufficiently large.

The proof of part (c) is deferred to Section 6.1.

5.8. The obstruction section and the gluing map. We now put the results of this section together. Fix $h \in(0,1)$; let $r_{0}, \varepsilon$ be as in Proposition 5.7, and fix $r>r_{0}$.

Definition 5.8. Given $T_{-}, T_{+} \geq 5 r$ and $\Sigma \in \mathcal{M}$, define the " $\left(T_{-}, T_{+}\right)$-gluing along $\Sigma$ ", denoted by $u\left(T_{-}, T_{+}, \Sigma\right)$, to be the deformed pregluing (5.7), where $\psi_{\Sigma}$ is given by Proposition 5.7 and $\psi_{ \pm}$are given by Proposition 5.6.

Let $\mathcal{O} \rightarrow \times_{2}[5 r, \infty) \times \mathcal{M}$ denote the pullback of the obstruction bundle from Section I.2.3. This means that the fiber over $\left(T_{-}, T_{+}, \Sigma\right)$ is

$$
\mathcal{O}_{\left(T_{-}, T_{+}, \Sigma\right)}=\operatorname{Hom}\left(\operatorname{Coker}\left(D_{\Sigma}\right), \mathbb{R}\right) .
$$

Definition 5.9. Define a section $\mathfrak{s}: \times_{2}[5 r, \infty) \times \mathcal{M} \rightarrow \mathcal{O}$ as follows: If $\sigma \in \operatorname{Coker}\left(D_{\Sigma}\right)$, then

$$
\mathfrak{s}\left(T_{-}, T_{+}, \Sigma\right)(\sigma):=\left\langle\sigma, \mathcal{F}_{\Sigma}\left(\psi_{\Sigma}\right)\right\rangle,
$$

where $\psi_{\Sigma}$ is the solution to $(5.37)$ given by Proposition 5.7. 
Note that under the identification $\operatorname{Hom}\left(\operatorname{Coker}\left(D_{\Sigma}\right), \mathbb{R}\right) \simeq \operatorname{Coker}\left(D_{\Sigma}\right)$ given by the inner product, we have

$$
\mathfrak{s}\left(T_{-}, T_{+}, \Sigma\right)=\Pi \mathcal{F}_{\Sigma}\left(\psi_{\Sigma}\right) .
$$

Thus by (5.38), $u\left(T_{-}, T_{+}, \Sigma\right)$ is $J$-holomorphic if and only if $\mathfrak{s}\left(T_{-}, T_{+}, \Sigma\right)=0$.

Recall that $\alpha_{+}$denotes the list of Reeb orbits corresponding to the positive ends of $U_{+}$, and $\alpha_{-}$denotes the list of Reeb orbits corresponding to the negative ends of $U_{-}$. We now have a well-defined "gluing map"

$$
G: \mathfrak{s}^{-1}(0) \longrightarrow \mathcal{M}^{J}\left(\alpha_{+}, \alpha_{-}\right),
$$

sending $\left(T_{-}, T_{+}, \Sigma\right) \in \mathfrak{s}^{-1}(0)$ to $u\left(T_{-}, T_{+}, \Sigma\right)$.

The next two sections establish important properties of the obstruction section $\mathfrak{s}$ and the gluing map $G$.

\section{Properties of the obstruction section}

Continue with the gluing setup from Section 5.1. In this section we prove that the obstruction section $\mathfrak{s}$ defined in Section 5.8 is continuous. We also show that the restriction of $\mathfrak{s}$ to the set of triples $\left(T_{-}, T_{+}, \Sigma\right)$, such that the branched cover $\Sigma$ has only simple ramification points, is smooth. Finally, we show that if $J$ is generic, then all zeroes of $\mathfrak{s}$ occur in the latter set.

6.1. Proof that $\psi_{\Sigma}$ is Lipschitz. We begin with the previously deferred:

Proof of Proposition 5.7(c). Smoothness of $\psi_{\Sigma}$ off of the ramification points follows by standard elliptic bootstrapping using (5.37).

To prove that $\psi_{\Sigma}$ is Lipschitz near a given ramification point, identify a neighborhood of the ramification point in $\Sigma$ with a disc of radius $2 \rho>0$ in $\mathbb{C}$, via a holomorphic local coordinate $u$ for which $\pi^{*} z=z_{0}+u^{q+1}$. On this disc, equation (5.37) asserts that

$$
D_{\Sigma} \psi_{\Sigma}+F_{\Sigma}\left(\psi_{\Sigma}\right)=\eta
$$

where $\eta$ is some element of $\operatorname{Ker}\left(D_{\Sigma}^{*}\right)$, and therefore smooth. It follows using (5.22) that $\psi_{\Sigma}$ obeys an equation of the form

$$
\frac{\partial \psi_{\Sigma}}{\partial \bar{u}}+\left(\frac{\bar{u}}{u}\right)^{q} a\left(u, \psi_{\Sigma}\right) \frac{\partial \psi_{\Sigma}}{\partial u}+g\left(u, \psi_{\Sigma}\right)=0
$$

where $a(u, w)$ is the function depicted in (5.22), and $g(u, w)$ is a smooth function of its arguments.

To simplify the above equation, let $a_{0}:=a\left(0, \psi_{\Sigma}(0)\right)$, and introduce a Lipschitz change of coordinates to

$$
v:=u\left(1-a_{0}\left(\frac{\bar{u}}{u}\right)^{q+1}\right)^{1 /(q+1)} .
$$


This change of coordinates is invertible if $\left|a_{0}\right|$ is small, which we can arrange by taking $\varepsilon$ small in Proposition 5.7.

We now prove that $\psi_{\Sigma}$ is Lipschitz at $v=0$. To do so, define a function $\lambda$ of $v$, defined on the disc of radius $\rho$, by writing

$$
\psi_{\Sigma}(u)=\lambda(v(u))+\psi_{\Sigma}(0)-g\left(0, \psi_{\Sigma}(0)\right) \bar{u} .
$$

Application of the chain rule finds that $\lambda$ obeys an equation of the form

$$
\frac{\partial \lambda}{\partial \bar{v}}+\mathfrak{f}_{1} \frac{\partial \lambda}{\partial v}+\mathfrak{f}_{0}=0
$$

Here each of $\mathfrak{f}_{1}$ and $\mathfrak{f}_{0}$ can be written as a product $\mathfrak{m}(v) \cdot \mathfrak{b}(v, \lambda(v))$, where $|\mathfrak{m}| \leq c$, while $\mathfrak{b}$ is a smooth function of two variables with $\mathfrak{b}(0,0)=0$, whose derivatives have bounds depending only on $u_{+}, u_{-}$, and $\eta$.

As a consequence of $(6.2)$, for $|v|<\rho$ the function $\lambda$ can be written as

$$
\lambda(v)=\left.\frac{1}{\pi} \int_{|x|<\rho} \frac{1}{v-x}\left(\mathfrak{f}_{1} \frac{\partial \lambda}{\partial v}+\mathfrak{f}_{0}\right)\right|_{x}+\lambda_{0}(v),
$$

where $\lambda_{0}$ is a holomorphic function with $\left|d \lambda_{0}(v)\right|<c \rho^{-1}$ for $|v|<\rho / 2$.

Recall from Lemma 5.3 that $\psi_{\Sigma}$ is a Holder continuous function of $u$. Since $\lambda(0)=0$, it follows that there exists $\sigma^{\prime} \in(0,1]$ such that $|\lambda(v)| \leq c_{\sigma^{\prime}}|v|^{\sigma^{\prime}}$ where $c_{\sigma^{\prime}}$ is a constant. Let $\sigma$ denote the supremum of the set of such $\sigma^{\prime}$. Then for $\sigma^{\prime} \in(0, \sigma)$, we know that $\left|\mathfrak{f}_{1}\right|,\left|\mathfrak{f}_{0}\right| \leq c c_{\sigma^{\prime}}|v|^{\sigma^{\prime}}$.

To bound $|\lambda(v)|$, fix $\epsilon \in(0, \sigma)$. Since $\lambda(0)=0$, we can subtract the instances of equation (6.3) for $\lambda(v)$ and $\lambda(0)$ to find that if $|v|<\rho / 2$, then

$$
|\lambda(v)| \leq c c_{\sigma-\epsilon} \int_{|x|<\rho} \frac{|v|}{|v-x||x|}|x|^{\sigma-\epsilon}|d \lambda|+c \rho^{-1}|v| .
$$

To bound the integral in (6.4), first consider the integral over an annulus $2^{n}|v|<|x| \leq 2^{n+1}|v|$, where $n$ is a positive integer with $2^{n}|v|<\rho$. The contribution from this annulus is at most

$$
c|v|\left(2^{n}|v|\right)^{-2+\sigma-\epsilon} \int_{2^{n}|v|<|x| \leq 2^{n+1}|v|}|d \lambda|,
$$

which by Holder's inequality is

$$
\leq c|v|\left(2^{n}|v|\right)^{-1+\sigma-\epsilon}\left(\int_{2^{n}|v|<|x| \leq 2^{n+1}|v|}|d \lambda|^{2}\right)^{1 / 2} .
$$

It follows from the definition of the norm on $\mathcal{H}_{\Sigma}$ that the integral in (6.5) is at most $\left(2^{n+1}|v|\right)^{1 / 2}\left\|\psi_{\Sigma}\right\|_{*}$. As a consequence, (6.5) is no greater than $c\left(2^{n}\right)^{-3 / 4+\sigma-\epsilon}|v|^{\sigma-\epsilon+1 / 4}$. Summing up these annular contributions, we find that the contribution to the integral in (6.4) from the region where $|x|>2|v|$ is at most

$$
c|v|^{\sigma-\epsilon+1 / 4} \sum_{n \geq 1,2^{n}|v|<\rho}\left(2^{\kappa}\right)^{n}, \quad \text { where } \kappa:=-3 / 4+\sigma-\epsilon .
$$


To bound the integral in (6.4) over the region where $|x|<|v| / 2$, divide this region into annuli of the form $2^{-n-1}|v| \leq|x| \leq 2^{-n}|v|$, where $n$ is a positive integer. By the same trick as before, the contribution from the $n$th such annulus is at most $c\left(2^{-n}|v|\right)^{\sigma-\epsilon+1 / 4}$, so the sum of these contributions is at most $c|v|^{\sigma-\epsilon+1 / 4}$. Finally, the integral over the region where $|v| / 2<$ $|x|<2|v|$ satisfies a bound of the same form, as we can see by considering annuli centered where $x=v$.

The conclusion from the above calculations is that if $|v|<\rho / 2$, then

$$
|\lambda(v)| \leq c c_{\sigma-\epsilon}|v|^{\sigma-\epsilon+1 / 4}\left(1+\sum_{n \geq 1,2^{n}|v|<\rho}\left(2^{\kappa}\right)^{n}\right)+c \rho^{-1}|v|,
$$

where $\kappa=-3 / 4+\sigma-\epsilon$. It follows from (6.6) that $\sigma$ must equal 1. Indeed, if $\sigma$ were less than 1 , then one could choose $\epsilon$ so that $\sigma^{\prime}:=\sigma-\epsilon+1 / 4$ is greater than $\sigma$ and less than 1. Then $\kappa$ would be negative, so (6.6) would give $|\lambda(v)| \leq$ const $\cdot|v|^{\sigma^{\prime}}$, contradicting the maximality of $\sigma$.

Granted that $\sigma=1$, pick any $\epsilon \in(0,1 / 4)$, and do the sum in (6.6) to see that $|\lambda(v)| \leq c_{0}|v|$, where $c_{0}$ has an a priori upper bound in terms of $\eta$ and the parameters used in the gluing. This proves that $\lambda$ is Lipschitz at $v=0$, and thus $\psi_{\Sigma}$ is Lipschitz at $u=0$.

To prove that $\lambda$ is Lipschitz at $v=w \neq 0$, introduce a new coordinate function

$$
v^{\prime}:=v-w-\mathfrak{f}_{1}(w) \cdot(\bar{v}-\bar{w}) .
$$

Viewed as a function of $v^{\prime}$, the function

$$
\lambda^{\prime}:=\lambda-\lambda(w)+\mathfrak{f}_{0}(w) \cdot(\bar{v}-\bar{w})
$$

obeys an equation of the form

$$
\frac{\partial \lambda^{\prime}}{\partial \overline{v^{\prime}}}+\mathfrak{f}_{1}^{\prime} \frac{\partial \lambda^{\prime}}{\partial v^{\prime}}+\mathfrak{f}_{0}^{\prime}=0
$$

where $\mathfrak{f}_{1}^{\prime}$ and $\mathfrak{f}_{0}^{\prime}$ have the same properties as do their unprimed counterparts in (6.2). Given this, a repeat of the arguments just given to prove that $\lambda$ is Lipschitz at $v=0$ proves that $\lambda^{\prime}$ is Lipschitz at $v^{\prime}=0$. As the Lipschitz constant that appears in the latter argument has a uniform bound, this proves that $\lambda$ is Lipschitz on a neighborhood of $v=0$, and thus that $\psi_{\Sigma}$ is Lipschitz near $u=0$.

\subsection{Continuity of the obstruction section.}

Proposition 6.1. The section $\mathfrak{s}: \times_{2}[5 r, \infty) \times \mathcal{M} \rightarrow \mathcal{O}$ is continuous.

Proof. Consider a smooth map from a neighborhood of 0 in $\mathbb{R}$ to $\times_{2}[5 r, \infty) \times$ $\mathcal{M}$. We will show that the restriction of the section to this path is continuous at 0 . The proof has four steps. 
Step 1. We first recall the precise meaning of continuity in this context. For $\tau \in \mathbb{R}$, denote the domain of the associated branched cover in $\mathcal{M}$ by $\Sigma_{\tau}$, and set $\Sigma:=\Sigma_{0}$. Identify each domain $\Sigma_{\tau}$ with $\Sigma$ via a path of diffeomorphisms $\tau \mapsto \varphi_{\tau}: \Sigma \rightarrow \Sigma_{\tau}$. The diffeomorphism $\varphi_{\tau}$ identifies the projection $\Sigma_{\tau} \rightarrow \mathbb{R} \times S^{1}$ with a map $\pi_{\tau}: \Sigma \rightarrow \mathbb{R} \times S^{1}$. We can choose the diffeomorphisms $\varphi_{\tau}$ so that $\varphi_{0}$ is the identity map, and so that $\pi_{\tau}$ agrees with $\pi:=\pi_{0}$ on the ends of $\Sigma$.

Now identify $T^{0,1} \Sigma_{\tau}$ with $T^{0,1} \Sigma$ as follows. First, use the diffeomorphism $\varphi_{\tau}$ to pull back $T^{0,1} \Sigma_{\tau}$ to a subbundle of $T^{*} \Sigma \otimes_{\mathbb{R}} \mathbb{C}$. Then use orthogonal projection with respect to the metric on $\Sigma$ to identify the latter subbundle with $T^{0,1} \Sigma$.

Under the above identifications, the kernel of $D_{\Sigma_{\tau}}^{*}$ defines a subspace $W_{\tau}$ of the space of $L^{2}$ sections of $T^{0,1} \Sigma$. Standard perturbation theory for linear operators shows that $W_{\tau}$ varies smoothly with $\tau$. In particular, for $\tau$ close to 0 in $\mathbb{R}$, orthogonal projection identifies $W_{\tau}$ with $W$. Indeed, this is how the vector bundle structure on $\mathcal{O}$ is defined.

Now for $|\tau|$ small, the section $\mathfrak{s}$ defines a vector in $W_{\tau}$, which is identified with a vector $\mathfrak{s}_{\tau} \in W$. More explicitly,

$$
\mathfrak{s}_{\tau}=\Pi^{\tau} \mathcal{F}^{\tau}\left(\psi_{\tau}\right),
$$

where $\Pi^{\tau}$ denotes the composition of the projection to $W_{\tau}$ with the identification $W_{\tau} \rightarrow W ; \mathcal{F}^{\tau}$ is shorthand for the map $\mathcal{F}_{\Sigma_{\tau}}$ in $(5.36)$; and $\psi_{\tau}:=\psi_{\Sigma_{\tau}}$ is given by Proposition 5.7 applied to the triple $\left(T_{-}(\tau), T_{+}(\tau), \Sigma_{\tau}\right)$. We want to show that the map $\tau \mapsto \mathfrak{s}_{\tau}$ is continuous at 0 .

Step 2. We now study the $\tau$ dependence of the various parts of (6.7). Since the subspace $W_{\tau}$ varies smoothly with $\tau$, so does the projection $\Pi^{\tau}$. Next, for any $\psi \in \mathcal{B}_{\Sigma_{\tau}}$, not necessarily the one given by Proposition 5.7, write

$$
\mathcal{F}^{\tau}(\psi)=F^{\tau}(\psi)+\mathcal{G}^{\tau}(\psi),
$$

where $F^{\tau}(\psi)$ is shorthand for $F_{\Sigma_{\tau}}^{\prime}(\psi)$. Thus $\mathcal{G}^{\tau}(\psi)$ is the sum of all but the first two terms on the right hand side of the $\tau$ version of equation (5.13), with $\psi_{\Sigma}=\psi$ and with $\psi_{ \pm}$given by Proposition 5.6. By equation (5.13) and Proposition 5.6, the assignment $(\tau, \psi) \mapsto \mathcal{G}^{\tau}(\psi)$ defines a smooth function from a neighborhood of 0 in $\mathbb{R}$ cross $\mathcal{H}_{\Sigma}$ to $\mathcal{H}_{0}(\Sigma)$. The function $(\tau, \psi) \mapsto F^{\tau}(\psi)$ is not necessarily smooth, but we have the following weaker statement:

Lemma 6.2. For $\psi_{0}=\psi_{\Sigma_{0}}$ given by Proposition 5.7 at $\tau=0$, we have

$$
\lim _{\tau \rightarrow 0}\left\|F^{\tau}\left(\psi_{0}\right)-F^{0}\left(\psi_{0}\right)\right\|=0 .
$$

Step 3. Assuming Lemma 6.2, we now complete the proof of Proposition 6.1. By Lemma 6.2 and the other conclusions of Step 2, it is enough to show that $\psi_{\tau} \in \mathcal{H}_{\Sigma}$ is a continuous function of $\tau$ at $\tau=0$. 
To prove the latter statement, recall that $\psi_{\tau}$ is the fixed point of a contraction mapping $\mathcal{I}^{\tau}$, which is defined as in (5.39) but with all the terms depending on $\tau$. Thus

$$
\begin{aligned}
\left\|\psi_{\tau}-\psi_{0}\right\|_{*} & =\left\|\mathcal{I}^{\tau}\left(\psi_{\tau}\right)-\mathcal{I}^{0}\left(\psi_{0}\right)\right\|_{*} \\
& \leq\left\|\mathcal{I}^{\tau}\left(\psi_{\tau}\right)-\mathcal{I}^{\tau}\left(\psi_{0}\right)\right\|_{*}+\left\|\mathcal{I}^{\tau}\left(\psi_{0}\right)-\mathcal{I}^{0}\left(\psi_{0}\right)\right\|_{*} .
\end{aligned}
$$

The contraction property of $\mathcal{I}^{\tau}$ asserts that

$$
\left\|\mathcal{I}^{\tau}\left(\psi_{\tau}\right)-\mathcal{I}^{\tau}\left(\psi_{0}\right)\right\|_{*} \leq \frac{1}{2}\left\|\psi_{\tau}-\psi_{0}\right\|_{*}
$$

for all $\tau$. Meanwhile, Lemma 6.2 and the other conclusions of Step 2 imply that

$$
\lim _{\tau \rightarrow 0}\left\|\mathcal{I}^{\tau}\left(\psi_{0}\right)-\mathcal{I}^{0}\left(\psi_{0}\right)\right\|_{*}=0 .
$$

It follows from (6.8) to (6.10) that $\lim _{\tau \rightarrow 0}\left\|\psi_{\tau}-\psi_{0}\right\|_{*}=0$.

Step 4. We now prove Lemma 6.2. Let $\psi=\psi_{\Sigma_{0}}$ be the function given by Proposition 5.7 at $\tau=0$. Away from the ramification points of $\pi$, the function $F^{\tau}(\psi)$ varies smoothly with $\tau$. The only difficulty arises from the variation of $F^{\tau}(\psi)$ near the ramification points.

To understand the latter, it proves convenient to choose the diffeomorphisms $\varphi_{\tau}$ in Step 1 to have two additional properties that concern each critical point $p$ of $\pi$. First, there is a neighborhood of $p$ in $\Sigma$ on which $\varphi_{\tau}$ sends the complex structure on $\Sigma$ to the complex structure on $\Sigma_{\tau}$. Second, there is a holomorphic coordinate $u$ identifying a smaller neighborhood of $p$ with the disk of radius $R>0$ in $\mathbb{C}$, such that the projection $\pi_{\tau}$ in this neighborhood is given by

$$
\pi_{\tau}(u)=z_{p}+u^{q+1}+b_{q-1} u^{q-1}+\cdots+b_{0},
$$

where each $b_{j}$ varies smoothly with $\tau$ and vanishes at $\tau=0$.

In this neighborhood, as in equation (5.22), we have

$$
F^{\tau}(\psi)=\frac{\partial_{\bar{u}} \overline{\pi_{\tau}}}{\partial_{u} \pi_{\tau}} a\left(\pi_{\tau}^{*} t, \psi\right) \partial_{u} \psi d \bar{u}+P\left(\pi_{\tau}^{*} t, \psi\right)
$$

where $P(t, w)$ is a smooth function of its arguments. So to prove Lemma 6.2, it suffices to show that for all $\varepsilon_{1}>0$, there exists $\varepsilon_{2}>0$ such that if $|\tau|<\varepsilon_{2}$, if $\rho \in(0, R / 4)$ and if $u_{0} \in \mathbb{C}$ with $\left|u_{0}\right| \leq R / 4$, then

$$
\rho^{-v} \int_{\left|u-u_{0}\right|<\rho}\left|\frac{\partial_{\bar{u}} \overline{\pi_{\tau}}}{\partial_{u} \pi_{\tau}} a\left(\pi_{\tau}^{*} t, \psi\right)-\left(\frac{\bar{u}}{u}\right)^{q} a\left(\pi_{0}^{*} t, \psi\right)\right|^{2}\left|\partial_{u} \psi\right|^{2}<\varepsilon_{1} .
$$


Recall from Proposition 5.7(c) that $\psi$ is Lipschitz. Also, the function $a$ and its derivatives are uniformly bounded. Hence, it is enough to show that

$$
\rho^{-v} \int_{\left|u-u_{0}\right|<\rho}\left|\frac{\partial_{\bar{u}} \overline{\pi_{\tau}}}{\partial_{u} \pi_{\tau}}-\left(\frac{\bar{u}}{u}\right)^{q}\right|^{2}
$$

can be made as small as desired by taking $|\tau|>0$ sufficiently small.

To prove this, note that for $|u|<R$ and for $|\tau|$ small, $\left|\partial_{u} \pi_{\tau}-(q+1) u^{q}\right| \leq$ const $\cdot|\tau|$. It follows that for any $\varepsilon_{3}>0$, the integrand in (6.13) is greater than $\varepsilon_{3}$ only where $|u| \leq$ const $\cdot|\tau|^{1 / q} \varepsilon_{3}^{-1 / 2 q}$. The contribution to (6.13) outside of this region is bounded by a constant multiple of $\varepsilon_{3}$. Since the integrand in (6.13) is uniformly bounded, the remaining contribution to (6.13) is at most a constant multiple of $\left(|\tau|^{1 / q} \varepsilon_{3}^{-1 / 2 q}\right)^{2-v}$, which can be made arbitrary small by taking $|\tau|$ sufficiently small with respect to $\varepsilon_{3}$. This completes the proof of Lemma 6.2 and Proposition 6.1.

6.3. Smoothness of the obstruction section. The moduli space $\mathcal{M}$ of branched covers has a natural stratification defined as follows. For $k=$ $0, \ldots, N_{+}+N_{-}-2$, let $\mathcal{M}_{(k)}$ denote the set of $\Sigma \in \mathcal{M}$ for which the set of ramification points in $\Sigma$ has cardinality $N_{+}+N_{-}-2-k$. In particular, $\mathcal{M}_{(0)}$ is an open dense subset of $\mathcal{M}$, consisting of branched covers in which every ramification point $p$ is simple, meaning that the projection $\pi: \Sigma \rightarrow \mathbb{R} \times S^{1}$ is described in local coordinates near $p$ by $\pi(u)=z_{p}+u^{2}$. The set $\mathcal{M}_{(k)}$ is a complex manifold of complex dimension $N_{+}+N_{-}-2-k$.

Lemma 6.3. For each $k$, the restriction of $\mathfrak{s}$ to $\times_{2}[5 r, \infty) \times \mathcal{M}_{(k)}$ is smooth.

Proof. This follows from a slight upgrading of the proof of Proposition 6.1, so we will carry over the notation from that proof. Consider a smooth map from a neighborhood of 0 in $\mathbb{R}$ to $\times_{2}[5 r, \infty) \times \mathcal{M}_{(k)}$. We want to prove that the expression in (6.7) varies smoothly with $\tau$, where $\psi_{\tau}$ is the fixed point of the contraction mapping $\mathcal{I}^{\tau}$. For this purpose it is enough to show that $\mathcal{I}^{\tau}$ varies smoothly with $\tau$. The only missing step is to show that the function $(\tau, \psi) \mapsto F^{\tau}(\psi)$ is smooth. Since our path stays in a fixed stratum $\mathcal{M}_{(k)}$, the polynomials $\pi_{\tau}$ in (6.11) must have the form

$$
\pi_{\tau}(u)=z_{p}+u^{q+1}+b_{0}(\tau) .
$$

Then $\partial_{u} \pi_{\tau}$ is independent of $\tau$, so the ratio $\partial_{\bar{u}} \overline{\pi_{\tau}} / \partial_{u} \pi_{\tau}$ that appears in (6.12) does not depend on $\tau$. It follows from (6.12) that $F$ is smooth as required.

6.4. Zeroes of $\mathfrak{s}$ have simple ramification points when $J$ is generic.

Lemma 6.4. If $J$ is generic, then all zeroes of $\mathfrak{s}$ are contained in the open stratum $\times_{2}[5 r, \infty) \times \mathcal{M}_{(0)}$. 
Proof. The proof has four steps.

Step 1. Here is the setup: Fix $\left(T_{-}, T_{+}, \Sigma\right) \in \mathfrak{s}^{-1}(0)$, and let $C$ denote the corresponding $J$-holomorphic curve produced by the gluing construction. By Theorem 4.1, we can assume that $J$ is generic so that $C$ is unobstructed and immersed. Fix $\delta>0$ very small and $l \gg 2$, and let $\mathcal{U}$ denote a small neighborhood of $J$ in the space of $C^{l}$ admissible almost complex structures $J^{\prime}$ that agree with $J$ within distance $\delta$ of $u_{-}$and $u_{+}$. We then have a universal moduli space $\mathcal{C}$ consisting of pairs $\left(J^{\prime}, C^{\prime}\right)$ where $J^{\prime} \in \mathcal{U}$ and $C^{\prime}$ is a $J^{\prime}$-holomorphic curve that is a deformation of $C$. Let $\mathcal{Z} \subset \mathcal{C}$ denote the set of pairs $\left(J^{\prime}, C^{\prime}\right)$ such that $C^{\prime}$ is obtained by the $J^{\prime}$ version of the gluing construction from a zero of $\mathfrak{s}$ on $\times_{2}[5 r, \infty) \times \mathcal{M}_{(k)}$ with $k>0$. Note that $\mathcal{Z}$ is invariant under the $\mathbb{R}$-action on $\mathcal{C}$. As in our previous genericity arguments, it is then enough to show that if $\delta>0$ is sufficiently small, then $\mathcal{Z}$ is a codimension 2 subvariety of $\mathcal{C}$.

Step 2. Fix a point $p \in C$ arising from a ramification point of $\Sigma$ under the gluing construction. Given $\left(J^{\prime}, C^{\prime}\right)$ in a small neighborhood $\mathcal{N}$ of $(J, C)$ in $\mathcal{C}$, we now describe the local structure of $C^{\prime}$ near $p$.

Let $B$ denote a disc containing the origin in $\mathbb{C}$ with coordinate $v$. For each $\left(J^{\prime}, C^{\prime}\right)$, fix a smooth embedding $\varphi_{\left(J^{\prime}, C^{\prime}\right)}: B \rightarrow C^{\prime}$, such that $\varphi_{(J, C)}$ maps $B$ into a neighborhood of $p$, and such that $\varphi_{\left(J^{\prime}, C^{\prime}\right)}$ depends smoothly on $\left(J^{\prime}, C^{\prime}\right)$.

Recall our local coordinates $z$ and $w$ in a neighborhood of $\mathbb{R} \times \alpha$ from Section 2.1. It follows from $(2.1)$ that given $\left(J^{\prime}, C^{\prime}\right)$, the function $x:=\varphi_{\left(J^{\prime}, C^{\prime}\right)}^{*}$ on $B$ obeys the equation

$$
\frac{\partial x}{\partial \bar{v}}-a \frac{\partial \bar{x}}{\partial \bar{v}}=0
$$

As a consequence, for $\left(J^{\prime}, C^{\prime}\right)$ in a small neighborhood $\mathcal{N}$ of $(J, C)$ in $\mathcal{C}$, the function $x$ near $v=0$ has the form

$$
x=x_{0}+\left(1-\left|a_{0}\right|^{2}\right)^{-1}\left(\mathcal{P}+a_{0} \overline{\mathcal{P}}\right)+O\left(|v|^{q+2}\right),
$$

where $a_{0}$ denotes the value of the function $a$ at $v=0$, while $q$ denotes the ramification index of $p$ in $C$, and $\mathcal{P}$ is a holomorphic polynomial of degree $q+1$ whose coefficients depend smoothly on $\left(J^{\prime}, C^{\prime}\right)$. Moreover, the maps $\varphi_{\left(J^{\prime}, C^{\prime}\right)}$ can be chosen so that

$$
\mathcal{P}(v)=v^{q+1}+b_{q-1} v^{q-1}+\cdots+b_{0} .
$$

Ramification points in $C^{\prime}$ near $p$ correspond to roots of $\partial_{v} \mathcal{P}$.

The coefficients $b_{0}, \ldots, b_{q-1}$ identify $\mathcal{P}$ with an element of $\mathbb{C}^{q}$, so we have defined a smooth map $\mathcal{P}: \mathcal{N} \rightarrow \mathbb{C}^{q}$. There is a complex codimension 1 
subvariety $\Delta \subset \mathbb{C}^{q}$ such that $\partial_{v} \mathcal{P}$ has $q$ distinct roots if and only if the coefficients of $\mathcal{P}$ correspond to a point in $\mathbb{C}^{q} \backslash \Delta$. So by Step 1, to prove Lemma 6.4 , it suffices to show that the differential

$$
d \mathcal{P}_{(J, C)}: T_{(J, C)} \mathcal{C} \longrightarrow \mathbb{C}^{q}
$$

is surjective.

Step 3. To prepare for the proof that (6.15) is surjective, we now construct some useful tangent vectors in $T_{(J, C)} \mathcal{C}$.

As in Section 3.1, a tangent vector in $T_{(J, C)} \mathcal{C}$ consists of a pair $(j, \zeta)$, where $j$ is a $(0,1)$ bundle automorphism of $T(\mathbb{R} \times Y)$, and $\zeta$ is an $L_{1}^{2}$ section of the normal bundle $N_{C}$, such that

$$
D_{C} \zeta=j_{C} .
$$

Here $D_{C}: C^{\infty}\left(N_{C}\right) \rightarrow C^{\infty}\left(N_{C} \otimes T^{0,1} C\right)$ denotes the linear deformation operator associated to $C$, and $j_{C} \in \operatorname{Hom}^{0,1}(T C, N C)$ is defined in (3.7).

The operator $D_{C}$ can be described more explicitly near $p$ as follows. By choosing $B$ sufficiently small, we can assume that the vector field $\partial_{s}$ is not tangent to $C$ on the image of $B$. Over $B$, we can then use $\partial_{s}$ to trivialize $N_{C}$, and $d \bar{v}$ to trivialize $T^{0,1} C$. In these trivializations, if $f$ is a complex function on $B$, then

$$
D_{C} f=\partial_{\bar{v}} f+\nu_{C} f+\mu_{C} \bar{f}
$$

on $B$, where $\nu_{C}$ and $\mu_{C}$ are complex functions on $B$.

Now to construct some useful tangent vectors, let $\rho$ denote the diameter of $B$. Fix a smooth function $\beta:[0, \infty) \rightarrow[0,1]$ which equals 1 on $[0, \rho / 2]$ and 0 on $[\rho, \infty)$. Let $\mathcal{H}_{B}$ denote the space of $L_{1}^{2}$ functions on $B$ whose restriction to $\partial B$ is in the span of $\left\{\rho^{-p} v^{p}\right\}_{-\infty<p<q}$. Define an operator

$$
\begin{aligned}
D_{C, B} f: \mathcal{H}_{B} & \longrightarrow L^{2}(B ; \mathbb{C}), \\
f & \longmapsto \partial_{\bar{v}} f+\beta\left(\nu_{C} f+\mu_{C} \bar{f}\right) .
\end{aligned}
$$

Lemma 6.5. The operator $D_{C, B}$ in (6.16) is Fredholm. Its index is $2 q$ and its cokernel is trivial. Its kernel has a basis $\left\{f_{k, A}\right\}_{0 \leq k<q, A \in\{0,1\}}$ such that

$$
f_{k, A}=i^{A} v^{k}+O\left(|v|^{k+1}\right)
$$

as $v \rightarrow 0$.

Proof. The operator $D_{C, B}$ differs from $\partial_{\bar{v}}$ by a zeroth order term. Since the latter is a Fredholm, index $2 q$ operator from $\mathcal{H}_{B}$ to $L^{2}(B ; \mathbb{C})$, so is $D_{C, B}$.

Note that each $f \in \operatorname{Ker}\left(D_{C, B}\right)$ extends from $B$ to the whole of $\mathbb{C}$ as a function that is holomorphic on $\mathbb{C} \backslash B$. Moreover, if $f$ is not identically zero, then $f$ behaves at large $|v|$ as $c v^{k}+O\left(|v|^{k-1}\right)$ with $c \neq 0$ and $k<q$. Finally, all zeroes of $f$ have positive multiplicity. It follows that $f$ has at most $q-1$ zeroes. This implies that $\operatorname{dim} \operatorname{Ker}\left(D_{C, B}\right) \leq 2 q$, and hence 
$\operatorname{Coker}\left(D_{C, B}\right)=\{0\}$. It also follows that a zero of a kernel element has multiplicity at most $q-1$, and this implies that $\operatorname{Ker}\left(D_{C, B}\right)$ has a basis of the desired form.

As in Section 3.1, there is a codimension 1 subvariety $B^{\prime} \subset B$, such that any $C^{k}$ function $f$ with support on the interior of $B$ can be realized as $j_{C}$ for some $j \in T_{J} \mathcal{U}$ for some $\delta>0$, provided that $f$ vanishes on a neighborhood of $B^{\prime}$. For $\varepsilon>0$ small, let $\chi_{\varepsilon}: B \rightarrow[0,1]$ be a smooth function which is 0 within distance $\varepsilon$ of $B^{\prime}$ and which is 1 where the distance to $B^{\prime}$ is $\geq 2 \varepsilon$. Fix a basis $\left\{f_{k, A}\right\}$ for $\operatorname{ker}\left(D_{C, B}\right)$ as in Lemma 6.5 , and choose $j_{\varepsilon, k, A} \in T_{\mathcal{J}} \mathcal{U}$ such that

$$
\left(j_{\varepsilon, k, A}\right)_{C}=\chi_{\varepsilon} D_{C}\left(\beta f_{k, A}\right) .
$$

To complete this to a tangent vector to $\mathcal{C}$, let $D_{C}^{-1}$ denote the unique right inverse of $D_{C}$ that maps to the $L^{2}$-orthogonal complement of $\operatorname{Ker}\left(D_{C}\right)$. Define

$$
\zeta_{\varepsilon, k, A}:=D_{C}^{-1}\left(\chi_{\varepsilon} D_{C}\left(\beta f_{k, A}\right)\right)+\left(\beta f_{k, A}\right)_{0},
$$

where $(\cdot)_{0}$ denotes $L^{2}$-orthogonal projection onto $\operatorname{Ker}\left(D_{C}\right)$. Then

$$
\left(j_{\varepsilon, k, A}, \zeta_{\varepsilon, k, A}\right) \in T_{(J, C)} \mathcal{C} .
$$

Define $\mathcal{T}_{\varepsilon}$ to be the span of the tangent vectors (6.19) for $0 \leq k<q$ and $A \in\{0,1\}$.

Step 4. We now complete the proof of Lemma 6.4. By Step 2, it suffices to show that if $\varepsilon>0$ is sufficiently small, then $d \mathcal{P}_{(J, C)}$ restricts to an isomorphism from $\mathcal{T}_{\varepsilon}$ to the space of polynomials of the form (6.14).

Note that for $(j, \zeta) \in T_{(J, C)} \mathcal{C}$, if $\zeta=c v^{k}+O\left(|v|^{k+1}\right)$ as $v \rightarrow 0$ with $c \neq 0$ and $k<q$, then

$$
d \mathcal{P}_{(J, C)}(j, \zeta)=c v^{k}+O\left(|v|^{k+1}\right) .
$$

If we could take $\varepsilon=0$ in (6.18), then we would be done by (6.17), since $\zeta_{\varepsilon, k, A}=\beta f_{k, A}$ when $\varepsilon=0$. The claim still holds when $\varepsilon>0$ is small, because $\zeta_{\varepsilon, k, A}$ converges in the $C^{\infty}$ topology on compact sets to $\beta f_{k, A}$ as $\varepsilon \rightarrow 0$.

\section{Bijectivity of the gluing map}

Continue with the setup and notation from Section 5. The goal of this section is to prove Theorem 7.3 below, which asserts roughly that the gluing map (5.44), applied to triples $\left(T_{-}, T_{+}, \Sigma\right)$ with $T_{-}, T_{+}$large, describes all curves in $\mathcal{M}_{J}\left(\alpha_{+}, \alpha_{-}\right)$that are "close to breaking" into $U_{+}$and $U_{-}$along branched covers of $\mathbb{R} \times \alpha$.

7.1. Statement of the gluing theorem. The set of curves that are "close to breaking" in the above sense is denoted by $\mathcal{G}_{\delta}\left(U_{+}, U_{-}\right)$. The precise 
definition of $\mathcal{G}_{\delta}\left(U_{+}, U_{-}\right)$in general was given in Definition I.1.10. We now recall this definition for convenience, using our standing assumptions (1.1) and (1.2) to recast it slightly.

We will use the following notation: If $\psi_{-}$is a section of the normal bundle to $u_{-}$with $\left|\psi_{-}\right|<\delta_{0}$, then $e_{-} \circ \psi_{-}$denotes the immersed surface in $\mathbb{R} \times Y$ whose domain is that of $u_{-}$, given by composing the section $\psi_{-}$with the exponential map $e_{-}$. If $\psi_{+}$is a section of the normal bundle to $u_{+}$with $\left|\psi_{+}\right|<\delta_{0}$, define $e_{+} \circ \psi_{+}$likewise.

Definition 7.1. For $\delta>0$, define $\widetilde{\mathcal{G}}_{\delta}\left(U_{+}, U_{-}\right)$to be the set of immersed (except possibly for finitely many singular points) surfaces in $\mathbb{R} \times Y$ that can be decomposed as $C_{-} \cup C_{0} \cup C_{+}$, such that the following hold:

- There is a real number $R_{-}$, and a section $\psi_{-}$of the normal bundle to $u_{-}$with $\left|\psi_{-}\right|<\delta$, such that $C_{-}$is the $s \mapsto s+R_{-}$translate of the $s \leq 1 / \delta$ part of $e_{-} \circ \psi_{-}$.

- Likewise, there is a real number $R_{+}$, and a section $\psi_{+}$of the normal bundle to $u_{+}$with $\left|\psi_{+}\right|<\delta$, such that $C_{+}$is the $s \mapsto s+R_{+}$translate of the $s \geq-1 / \delta$ part of $e_{+} \circ \psi_{+}$.

- $R_{+}-R_{-}>2 / \delta$.

- $C_{0}$ is a connected genus zero surface with boundary which is contained in the radius $\delta$ tubular neighborhood of $\mathbb{R} \times \alpha$, such that the tubular neighborhood projection $C_{0} \rightarrow \mathbb{R} \times \alpha$ is a branched covering. Moreover, $C_{0}$ has positive ends of multiplicities $a_{\bar{N}_{+}+1}, \ldots, a_{N_{+}}$, and negative ends of multiplicities $a_{-N_{-}-1}, \ldots, a_{-N_{-}}$.

- $\partial C_{0}=\partial C_{-} \sqcup \partial C_{+}$, where the positive boundary circles of $C_{-}$agree with the negative boundary circles of $C_{0}$, and the positive boundary circles of $C_{0}$ agree with the negative boundary circles of $C_{+}$.

Let $\mathcal{G}_{\delta}\left(U_{+}, U_{-}\right)$denote the set of surfaces $C \in \widetilde{\mathcal{G}}_{\delta}\left(U_{+}, U_{-}\right)$such that $C$ is $J$-holomorphic. Note that the definition implies that any element of $\mathcal{G}_{\delta}\left(U_{+}, U_{-}\right)$is in $\mathcal{M}^{J}\left(\alpha_{+}, \alpha_{-}\right)$and has index 2 .

Definition 7.2. Given $\delta>0$, define $\mathcal{U}_{\delta} \subset \times_{2}[5 r, \infty) \times \mathcal{M}$ to be the set of $\left(T_{-}, T_{+}, \Sigma\right)$ such that $u\left(T_{-}, T_{+}, \Sigma\right) \in \widetilde{\mathcal{G}}_{\delta}\left(U_{+}, U_{-}\right)$.

Theorem 7.3. Fix $h \in(0,1)$, and let $r_{0}, \varepsilon$ be as in Proposition 5.7. Then:

(a) If $R$ is sufficiently large with respect to $\delta$, then

$$
\times_{2}[R, \infty) \times \mathcal{M} \subset \mathcal{U}_{\delta} .
$$

(b) If $r>r_{0}$ is chosen sufficiently large and if $\delta>0$ is sufficiently small with respect to $r$, then the gluing map (5.44) restricts to a homeomorphism

$$
G: \mathfrak{s}^{-1}(0) \cap \mathcal{U}_{\delta} \stackrel{\simeq}{\longrightarrow} \mathcal{G}_{\delta}\left(U_{+}, U_{-}\right)
$$


Proof. Part (a) follows from Propositions 5.7(b) and 5.6(c). To prove part (b), we will show in Lemmas 7.4 and 7.5 below that if $r$ is sufficiently large and if $\delta$ is sufficiently small with respect to $r$, then the map (7.1) is a bijection. Continuity of the map (7.1) follows from Proposition 5.6(b) together with the proof of Proposition 6.1.

\subsection{Injectivity of the gluing map.}

Lemma 7.4. Fix $h \in(0,1)$. If $r>r_{0}$ is sufficiently large, and if $\delta>0$ is sufficiently small, then the restricted gluing map (7.1) is injective.

Proof. The proof has two steps.

Step 1. Fix $r>r_{0}$ and $\delta>0$, and let $\left(T_{-}, T_{+}, \Sigma\right) \in \mathcal{U}_{\delta}$. We now show that if $u\left(T_{-}, T_{+}, \Sigma\right)$ is $J$-holomorphic, then $u\left(T_{-}, T_{+}, \Sigma\right)$ determines $\Sigma$.

Choose a decomposition $C_{-} \cup C_{0} \cup C_{+}$of $u\left(T_{-}, T_{+}, \Sigma\right)$ as in Definition 7.1. Recall the coordinates $(z, w)$ on a tubular neighborhood of $\mathbb{R} \times \alpha$. Let $\mathfrak{p}$ : $C_{0} \rightarrow \mathbb{R} \times S^{1}$ denote the tubular neighborhood projection sending $(z, w) \mapsto$ $(z, 0)$. Since $C_{0}$ and the $z=$ constant disks are $J$-holomorphic, it follows that the map $\mathfrak{p}$ is a branched cover on (the domain of) $C_{0}$. As such, it pulls back the complex structure on $\mathbb{R} \times S^{1}$ to a complex structure $j$ on $C_{0}$ (which generally does not agree with the restriction of the almost complex structure $J$ on $\mathbb{R} \times Y)$. Let $\widetilde{\mathfrak{p}}:\left(\widetilde{C}_{0}, \widetilde{j}\right) \rightarrow \mathbb{R} \times S^{1}$ denote the element of $\mathcal{M}$ obtained by attaching half-infinite cylinders to the $\bar{N}_{+}$positive boundary circles and the $\bar{N}_{-}$negative boundary circles of $\left(C_{0}, j\right)$; the orderings and asymptotic markings of the resulting ends are induced from those of the negative ends of $u_{+}$and the positive ends of $u_{-}$, respectively, via the identification $\partial C_{0}=$ $\partial C_{+} \sqcup \partial C_{-}$.

We claim that $\widetilde{C}_{0}$ and $\Sigma$ (with their maps to $\mathbb{R} \times S^{1}$ and orderings and asymptotic markings of their ends) define the same element of $\mathcal{M}$. To see this, let $\Sigma_{0}$ be obtained from $\Sigma$ by removing the $s>R_{+}$part of the first $\bar{N}_{+}$positive ends and the $s<R_{-}$part of the first $\bar{N}_{-}$negative ends. The gluing construction defines a parameterization $f: \Sigma_{0} \stackrel{\simeq}{\longrightarrow} C_{0}$ with $\mathfrak{p} \circ f=\pi$. It follows from the definition of the complex structure $j$ on $C_{0}$ that the map $f$ is holomorphic with respect to $j$. Then $f$ extends to a biholomorphic map $\widetilde{f}: \Sigma \rightarrow\left(\widetilde{C}_{0}, \widetilde{j}\right)$, which satisfies $\widetilde{\mathfrak{p}} \circ \widetilde{f}=\pi$ and preserves the orderings and asymptotic markings of the ends.

Step 2. We now show that if $r>r_{0}$ is sufficiently large, if $\delta$ is sufficiently small, and if $u\left(T_{-}, T_{+}, \Sigma\right) \in \widetilde{\mathcal{G}}_{\delta}\left(U_{+}, U_{-}\right)$, then $T_{-}$and $T_{+}$are determined by $u\left(T_{-}, T_{+}, \Sigma\right)$. It suffices to prove the following two claims:

(i) If $r>r_{0}$ is sufficiently large, then for any given $R$, if $\delta$ is sufficiently small with respect to $R$, then $u\left(T_{-}, T_{+}, \Sigma\right) \in \widetilde{\mathcal{G}}_{\delta}\left(U_{+}, U_{-}\right)$implies $T_{-}, T_{+}>R$.

(ii) For any $r>r_{0}$, if $R$ is sufficiently large, if $T_{-}, T_{+}, T_{-}^{\prime}, T_{+}^{\prime}>R$ and if $u\left(T_{-}, T_{+}, \Sigma\right)=u\left(T_{-}^{\prime}, T_{+}^{\prime}, \Sigma\right)$, then $\left(T_{-}, T_{+}\right)=\left(T_{-}^{\prime}, T_{+}^{\prime}\right)$. 
Proof of (i). Given $p \in Y$ and $\rho>0$, let $B(p, \rho) \subset Y$ denote the ball of radius $\rho$ around $p$ in $Y$. If $\delta_{1}>0$ is sufficiently small, then there exist points $p_{-}, p_{+} \in Y$ with the following two properties: first, $p_{ \pm}$is contained in the projection of $u_{ \pm}$to $Y$. Second, $\mathbb{R} \times B\left(p_{-}, 2 \delta_{1}\right)$ does not intersect $u_{+}$, and $\mathbb{R} \times B\left(p_{+}, 2 \delta_{1}\right)$ does not intersect $u_{-}$. Fix $\delta_{1}$ and $p_{ \pm}$as above.

If $r>r_{0}$ is sufficiently large, then the estimates in Section 5 imply that for any $\left(T_{-}, T_{+}, \Sigma\right) \in \times_{2}(5 r, \infty) \times \mathcal{M}$, the sections $\psi_{ \pm}$produced by the gluing construction satisfy $\left|\psi_{ \pm}\left(p_{ \pm}\right)\right|<\delta_{1}$. Fix $r$ with this property.

Next, fix $\delta<\delta_{1}$, and suppose that $C:=u\left(T_{-}, T_{+}, \Sigma\right) \in \widetilde{\mathcal{G}}_{\delta}\left(U_{-}, U_{+}\right)$. Choose a decomposition $C=C_{-} \cup C_{0} \cup C_{+}$as in Definition 7.1. Let $a$ denote the supremum of $s$ on the intersection of $u_{-}$with $\mathbb{R} \times B\left(p_{-}, 2 \delta_{1}\right)$, and let $b:=s\left(p_{-}\right)$. It follows from the conditions on $C_{0}$ and $C_{+}$that any point in $C \cap\left(\mathbb{R} \times B\left(p_{-}, \delta_{1}\right)\right)$ must be in $C_{-}$, and hence must have $s \leq a+R_{-}+\delta_{1}$. On the other hand, since $C=u\left(T_{-}, T_{+}, \Sigma\right)$, it follows from our choice of $r$ that under the gluing construction, $p_{-}$gives rise to a point in $C \cap\left(\mathbb{R} \times B\left(p_{-}, \delta_{1}\right)\right)$ with $s \geq b+s_{-}-T_{-}-\delta_{1}$. Finally, the conditions in Definition 7.1 imply that $s_{-} \geq R_{-}+1 / \delta$. Combining the above inequalities, we find that $T_{-}$ is greater than $1 / \delta$ plus a constant depending only on $u_{-}$. Similarly, $T_{+}$is greater than $1 / \delta$ plus a constant depending only on $u_{+}$.

Proof of (ii). Given $x \in \mathbb{R}$, let $\Phi_{x}$ denote the automorphism of $\mathbb{R} \times Y$ sending $(s, y) \mapsto(s+x, y)$. We can find a point $p_{-}$in the $s \leq 0$ part of $u_{-}$, and a real number $0<\rho<\delta_{0}$, such that $\mathbb{R} \times B\left(p_{-}, \rho\right)$ does not intersect $u_{+}$, and such that the intersection of $u_{-}$with $\mathbb{R} \times B\left(p_{-}, \rho\right)$ is a single disc $B_{-}$on which the projection to $Y$ is an embedding. It follows from this last condition that there exist constants $c_{-}, \epsilon_{-}>0$ with the following property:

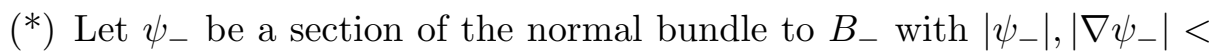
$\epsilon_{-}$. Then for any $x_{-} \in \mathbb{R}$ and for any $p_{-}^{\prime} \in B_{-}$, we have

$$
\operatorname{dist}\left(e_{-}\left(\psi_{-}\left(p_{-}\right)\right), \Phi_{x_{-}}\left(e_{-}\left(\psi_{-}\left(p_{-}^{\prime}\right)\right)\right)\right) \geq c_{-}\left|x_{-}\right| \text {. }
$$

Now fix $r>r_{0}$ and $R$. Let $T_{-}, T_{+}, T_{-}^{\prime}, T_{+}^{\prime}>R$ and suppose that $u\left(T_{-}, T_{+}, \Sigma\right)=u\left(T_{-}^{\prime}, T_{+}^{\prime}, \Sigma\right)$. Let $\psi_{-}$and $\psi_{-}^{\prime}$ denote the sections of the normal bundles to $u_{-T}$ and $u_{-T^{\prime}}$, respectively, coming from the gluing construction. Use the translations $\Phi_{T_{-}}$and $\Phi_{T_{-}^{\prime}}$ to regard both $\psi_{-}$and $\psi_{-}^{\prime}$ as sections of the normal bundle to $u_{-}$. By Propositions 5.6(c) and 5.7(b), there are constants $c, \lambda>0$ depending only on $u_{+}$and $u_{-}$such that $\left|\psi_{-}\right|,\left|\psi_{-}^{\prime}\right|<c \exp (-\lambda R)$. In particular, if $R$ is sufficiently large, then $\left|\psi_{-}\right|,\left|\psi_{-}^{\prime}\right|<\rho / 2$.

The point $p_{-}$in $u_{-}$gives rise to the point $\Phi_{s_{-}-T_{-}}\left(e_{-}\left(\psi_{-}\left(p_{-}\right)\right)\right)$in the gluing $u\left(T_{-}, T_{+}, \Sigma\right)$. Since this point is also in $u\left(T_{-}^{\prime}, T_{+}^{\prime}, \Sigma\right)$, there must exist $p_{-}^{\prime}$ in $B_{-}$with

$$
e_{-}\left(\psi_{-}\left(p_{-}\right)\right)=\Phi_{x_{-}}\left(e_{-}\left(\psi_{-}^{\prime}\left(p_{-}^{\prime}\right)\right)\right)
$$

where $x_{-}:=T_{-}-T_{-}^{\prime}$. 
Now the bound on $\left|\psi_{-}\right|$, together with the elliptic regularity in Lemma 2.2, leads to a bound of the same form on $\left|\nabla \psi_{-}\right|$. Hence, if $R$ is sufficiently large, then $(*)$ is applicable so that the inequality (7.2) holds.

On the other hand, by bounding the derivatives of the contraction mappings used to define $\psi_{-}$, one can show that $\psi_{-}$depends smoothly on $T_{+}$

and $T_{-}$, with $\left\|\frac{\partial \psi_{-}}{\partial T_{ \pm}}\right\| \leq c \exp (-\lambda R)$, where again $c, \lambda>0$ depend only on $u_{+}$and $u_{-}$. Therefore,

$$
\operatorname{dist}\left(e_{-}\left(\psi_{-}\left(p_{-}^{\prime}\right)\right), e_{-}\left(\psi_{-}^{\prime}\left(p_{-}^{\prime}\right)\right)\right) \leq c \exp (-\lambda R)\left(\left|x_{-}\right|+\left|x_{+}\right|\right),
$$

where $x_{+}:=T_{+}-T_{+}^{\prime}$. Combining this with (7.2) and (7.3), we obtain

$$
c_{-}\left|x_{-}\right| \leq c \exp (-\lambda R)\left(\left|x_{-}\right|+\left|x_{+}\right|\right) .
$$

By a symmetric argument, there is a constant $c_{+}$depending only on $u_{+}$ such that

$$
c_{+}\left|x_{+}\right| \leq c \exp (-\lambda R)\left(\left|x_{-}\right|+\left|x_{+}\right|\right) .
$$

If $R$ is sufficiently large, then the above two inequalities together imply that $x_{-}=x_{+}=0$, so that $\left(T_{-}, T_{+}\right)=\left(T_{-}^{\prime}, T_{+}^{\prime}\right)$.

\subsection{Surjectivity of the gluing map.}

Lemma 7.5. For fixed $h \in(0,1)$, if $r>r_{0}$ is chosen sufficiently large and if $\delta>0$ is sufficiently small with respect to $r$, then the restricted gluing map (7.1) is surjective.

Proof. The proof has three steps.

Step 1. Here is the setup: Let $C \in \mathcal{G}_{\delta}\left(U_{+}, U_{-}\right)$, and decompose $C=$ $C_{-} \cup C_{0} \cup C_{+}$as in Definition 7.1. Let $T_{-}$denote the real number for which the smallest critical value of $\left.s\right|_{C_{0}}$ is $R_{-}+T_{-}+1$. Let $T_{+}$denote the real number for which the largest critical value of $\left.s\right|_{C_{0}}$ is $R_{+}-T_{+}-1$. It follows from the conditions in Definition 7.1 that if $1 / \delta \geq 5 r+5$ (which we assume for the rest of this proof), then $T_{-}, T_{+} \geq 5 \mathrm{r}$. Also, as in the proof of Lemma 7.4, the decomposition of $C$ determines a branched cover $\Sigma$ in $\mathcal{M}$, with $s_{-}$close to $R_{-}+T_{-}$and $s_{+}$close to $R_{+}-T_{+}$.

The section $\psi_{-}$of the normal bundle to $u_{-}$given by Definition 7.1 determines a section of the normal bundle to $u_{-T}$, which we also denote by $\psi_{-}$, and which satisfies equation (5.24) on $u_{-T}^{\prime}$. Likewise, we have a section $\psi_{+}$ of the normal bundle to $u_{+T}$ satisfying equation (5.25) on $u_{+T}^{\prime}$. Part of the curve $C$ consists of the exponential map images of the sections $\psi_{ \pm}$over $u_{ \pm T}^{\prime}$. The rest of $C$ is described, in our coordinates $((s, t), w)$ on a tubular neighborhood of $\mathbb{R} \times \alpha$, by a map on $\Sigma^{\prime}$ sending $x \mapsto\left(\pi(x), \psi_{0}(x)\right)$ where $\psi_{0}$ is a complex-valued function on $\Sigma^{\prime}$. Let $\Sigma^{\prime \prime}$ be obtained from $\Sigma^{\prime}$ by removing the cylinders $\Sigma_{i}$. Let $\psi_{\Sigma}$ denote the restriction of $\psi_{0}$ to $\Sigma^{\prime \prime}$. Then $\psi_{\Sigma}$ satisfies equation (5.26) on $\Sigma^{\prime \prime}$. 
To show that $C$ is obtained from the gluing construction, we want to extend $\psi_{-}$over the rest of $u_{-T}$, extend $\psi_{+}$over the rest of $u_{+T}$ and extend $\psi_{\Sigma}$ over the rest of $\Sigma$, so that:

(i) Equation (5.24) holds on all of $u_{-T}$, equation (5.25) holds on all of $u_{+T}$ and equation (5.26) holds on all of $\Sigma$.

(ii) On each cylinder $\Sigma_{i}$ with $i<0$, we have

$$
\beta_{-}\left(\eta_{-T}+\psi_{-}\right)+\beta_{\Sigma} \psi_{\Sigma}=\psi_{0} .
$$

Likewise, on each cylinder $\Sigma_{i}$ with $i>0$, we have

$$
\beta_{+}\left(\eta_{+T}+\psi_{+}\right)+\beta_{\Sigma} \psi_{\Sigma}=\psi_{0}
$$

(iii) $\left\|\psi_{\Sigma}\right\|_{*}<\varepsilon$, where $\varepsilon$ is given by Proposition 5.7 .

(iv) $\psi_{-}$is orthogonal to the kernel of $D_{-}$, and $\psi_{+}$is orthogonal to the kernel of $D_{+}$.

Step 2. We now show that there exist $r_{1}>1$ and $\delta_{1}>0$ such that if $r>r_{1}$ and $\delta \leq \delta_{1}$, then $\psi_{ \pm}$and $\psi_{\Sigma}$ can be extended to satisfy conditions (i) to (iii) above. This step has two substeps.

Step 2.1. Consider one of the cylinders $\Sigma_{i}$, identified with $[A, B] \times \widetilde{S^{1}}$ with coordinates $(s, \tau)$. Here $\widetilde{S^{1}}$ denotes the $m$-fold cover of $S^{1}$, where $m$ is the degree of the restriction of the covering $\Sigma \rightarrow \mathbb{R} \times S^{1}$ to $\Sigma_{i}$. On this cylinder, the function $\psi:=\psi_{0}$ satisfies an equation of the form

$$
\left(\partial_{s}+L_{m}\right) \psi+F(\psi)=0
$$

where $F$ is type 1 quadratic in the sense of Definition 5.1. The purpose of this substep is to establish some properties of equation (7.6).

Recall that $L_{3 / 2}^{2}\left(\widetilde{S^{1}} ; \mathbb{R}^{2}\right)$ denotes the completion of the space of smooth $\mathbb{R}^{2}$-valued functions on $\widetilde{S^{1}}$ using the norm defined by

$$
\|\eta\|_{L_{3 / 2}^{2}}^{2}:=\int_{\widetilde{S^{1}}}\left\langle\eta,\left|L_{m}\right|^{3} \eta\right\rangle .
$$

Let $\Pi_{+}\left(\right.$resp. $\left.\Pi_{-}\right)$denote the $L^{2}$-orthogonal projection from $L_{3 / 2}^{2}\left(\widetilde{S^{1}} ; \mathbb{R}^{2}\right)$ to the span of the eigenvectors of $L_{m}$ with positive (resp. negative) eigenvalues.

Lemma 7.6. There exist constants $\epsilon_{0}>0$ and $c$ with the following property. Suppose $B-A \geq 1$ and $\epsilon<\epsilon_{0}$. Let $\lambda_{A} \in \Pi_{+} L_{3 / 2}^{2}\left(\widetilde{S^{1}} ; \mathbb{R}^{2}\right)$ and $\lambda_{B} \epsilon$ $\Pi_{-} L_{3 / 2}^{2}\left(\widetilde{S^{1}} ; \mathbb{R}^{2}\right)$ be given with $L_{3 / 2}^{2}$ norm less than $\epsilon$. Then there exists a unique solution $\psi$ to equation (7.6) on $[A, B] \times \widetilde{S^{1}}$ with $\|\psi\|_{L_{2}^{2}}<c \epsilon$ that satisfies the boundary conditions $\Pi_{+} \psi(A, \cdot)=\lambda_{A}$ and $\Pi_{-} \psi(B, \cdot)=\lambda_{B}$. 
Proof. Define a map

$$
\begin{aligned}
\mathcal{F}: L_{2}^{2}\left([A, B] \times \widetilde{S^{1}}\right) & \longrightarrow \Pi_{+} L_{3 / 2}^{2}\left(\widetilde{S^{1}} ; \mathbb{R}^{2}\right) \times \Pi_{-} L_{3 / 2}^{2}\left(\widetilde{S^{1}} ; \mathbb{R}^{2}\right) \times L_{1}^{2}\left([A, B] \times \widetilde{S^{1}}\right), \\
\psi & \longmapsto\left(\Pi_{+} \psi(A, \cdot), \Pi_{-} \psi(B, \cdot),\left(\partial_{s}+L_{m}\right) \psi+F(\psi)\right) .
\end{aligned}
$$

Calculations as in the proof of Lemma 3.7 show that the derivative of $\mathcal{F}$ at 0 is invertible, and the operator norm of its inverse has an upper bound independent of $B-A$. In addition, since $F$ is type 1 quadratic, it follows that

$$
\left\|\left(d \mathcal{F}_{\psi}-d \mathcal{F}_{0}\right) \xi\right\|_{L_{1}^{2}} \leq c\|\psi\|_{L_{2}^{2}}\|\xi\|_{L_{2}^{2}}
$$

where $c$ is independent of $B-A \geq 1$. The lemma now follows from the inverse function theorem.

Proof of Lemma 7.5, Step 2.2. Fix $i \in\left\{-1, \ldots,-\bar{N}_{-}\right\}$, and consider the problem of extending $\psi_{-}$over the $s \geq s_{-}-T_{-}$portion of the $i$ th positive end of $u_{-T}$ and extending $\psi_{\Sigma}$ over the $s \leq s_{i}$ portion of the $i$ th negative end of $\Sigma$. Both of these cylinders are identified with subcylinders of $\mathbb{R} \times \widetilde{S^{1}}$. Thus we need to find extensions of $\psi_{-}$over $\left[s_{-}-T_{-}, \infty\right) \times \widetilde{S^{1}}$, and of $\psi_{\Sigma}$ over $\left(-\infty, s_{i}\right] \times \widetilde{S^{1}}$, that satisfy the equations

$$
\begin{aligned}
\Theta_{-}\left(\psi_{-}, \psi_{\Sigma}\right) & =0, \quad s \geq s_{-}-T_{-}, \\
\Theta_{\Sigma}\left(\psi_{-}, \psi_{\Sigma}, 0\right) & =0, \quad s \leq s_{i},
\end{aligned}
$$

and that also satisfy equation (7.4) when $s_{-}-T_{-} \leq s \leq s_{i}$.

The following lemma provides solutions to equation (7.7).

Lemma 7.7. There exist constants $\epsilon_{0}>0, r_{1}>1$, and $c$ with the following property. Suppose $r>r_{1}$ and $\epsilon<\epsilon_{0}$. Let $\lambda_{-} \in \Pi_{+} L_{3 / 2}^{2}\left(\widetilde{S^{1}} ; \mathbb{R}^{2}\right)$ and $\lambda_{\Sigma} \epsilon$ $\Pi_{-} L_{3 / 2}^{2}\left(\widetilde{S^{1}} ; \mathbb{R}^{2}\right)$ be given with $L_{3 / 2}^{2}$ norm less than $\epsilon$. Then there exists a unique solution $\left(\psi_{-}, \psi_{\Sigma}\right)$ to (7.7) with $\left\|\psi_{-}\right\|_{L_{2}^{2}},\left\|\psi_{\Sigma}\right\|_{L_{2}^{2}}<c \epsilon$ that satisfies the boundary conditions $\Pi_{+} \psi_{-}\left(s_{-}-T_{-}, \cdot\right)=\lambda_{-}$and $\Pi_{-} \psi_{\Sigma}\left(s_{i}, \cdot\right)=\lambda_{\Sigma}$.

Proof. This is an application of the inverse function theorem similar to the proof of Lemma 7.6. In more detail, write $A:=s_{-}-T_{-}$and $B:=s_{i}$. Define a map

$$
\begin{gathered}
\mathcal{F}: L_{2}^{2}(s \geq A) \times L_{2}^{2}(s \leq B) \rightarrow \Pi_{+} L_{3 / 2}^{2} \times \Pi_{-} L_{3 / 2}^{2} \times L_{1}^{2}(s \geq A) \times L_{1}^{2}(s \leq B), \\
\left(\psi_{-}, \psi_{\Sigma}\right) \longmapsto\left(\Pi_{+} \psi_{-}(A, \cdot), \Pi_{-} \psi_{\Sigma}(B, \cdot), \Theta_{-}\left(\psi_{-}, \psi_{\Sigma}\right), \Theta_{\Sigma}\left(\psi_{-}, \psi_{\Sigma}, 0\right)\right) .
\end{gathered}
$$

The derivative of $\mathcal{F}$ at $(0,0)$ has the schematic form

$$
\begin{aligned}
\left(\psi_{-}, \psi_{\Sigma}\right) \longmapsto & \left(\Pi_{+} \psi_{-}(A, \cdot), \Pi_{-} \psi_{\Sigma}(B, \cdot),\right. \\
& \left.\left(\partial_{s}+L_{m}\right) \psi_{-}+a_{-}\left(\psi_{-}, \psi_{\Sigma}\right),\left(\partial_{s}+L_{m}\right) \psi_{\Sigma}+a_{\Sigma}\left(\psi_{-}, \psi_{\Sigma}\right)\right) .
\end{aligned}
$$


As in the proof of Lemma 7.6, the map (7.8), with the $a_{-}$and $a_{\Sigma}$ terms removed, is invertible, and the operator norm of its inverse is less than some constant $c_{0}$ which does not depend on $A$ and $B$. To prove that the map (7.8) itself is invertible, with its inverse bounded independently of $A$ and $B$, it is enough to show that

$$
\left\|a_{-}\left(\psi_{-}, \psi_{\Sigma}\right)\right\|_{L_{1}^{2}},\left\|a_{\Sigma}\left(\psi_{-}, \psi_{\Sigma}\right)\right\|_{L_{1}^{2}} \leq \frac{1}{4 c_{0}}\left(\left\|\psi_{-}\right\|_{L_{2}^{2}}+\left\|\psi_{\Sigma}\right\|_{L_{2}^{2}}\right) .
$$

The bound (7.9) follows directly from equations (5.11) and (5.13) and the decay estimates on $\eta_{-T}$, provided that $r$ is sufficiently large. It also follows from (5.11) and (5.13) that

$\left\|\left(d \mathcal{F}_{\left(\psi_{-}, \psi_{\Sigma}\right)}-d \mathcal{F}_{(0,0)}\right)\left(\xi_{-}, \xi_{\Sigma}\right)\right\|_{L_{1}^{2}} \leq c\left(\left\|\psi_{-}\right\|_{L_{2}^{2}}+\left\|\psi_{\Sigma}\right\|_{L_{2}^{2}}\right)\left(\left\|\xi_{-}\right\|_{L_{2}^{2}}+\left\|\xi_{\Sigma}\right\|_{L_{2}^{2}}\right)$. where $c$ is independent of $A$ and $B$ provided that $r \geq 1$. The lemma now follows from the inverse function theorem.

If $\delta$ is sufficiently small, then we can apply Lemma 7.7 with $\lambda_{-}=$ $\Pi_{+}\left(\psi_{0}-\eta_{-T}\right)\left(s_{-}-T_{-}, \cdot\right)$ and $\lambda_{\Sigma}=\Pi_{-} \psi_{0}\left(s_{i}, \cdot\right)$, to obtain a solution $\left(\psi_{-}, \psi_{\Sigma}\right)$ to equation $(7.7)$ satisfying the above boundary conditions and with $\left\|\psi_{-}\right\|_{L_{2}^{2}},\left\|\psi_{\Sigma}\right\|_{L_{2}^{2}}<c \delta$. By the uniqueness assertion in Lemma 7.6, equation (7.4) also holds, because by equation (5.14), both $\psi_{0}$ and $\beta_{-}\left(\eta_{-T}+\right.$ $\left.\psi_{-}\right)+\beta_{\Sigma} \psi_{\Sigma}$ satisfy equation (7.6).

By an analogous process, if $r$ is sufficiently large and $\delta$ is sufficiently small, then for $i=\left\{1, \ldots, \bar{N}_{+}\right\}$we can extend $\psi_{+}$over the $s \leq s_{+}+T_{+}$portion of the $i$ th negative end of $u_{+T}$ and extend $\psi_{\Sigma}$ over the $s \geq s_{i}$ portion of the $i$ th positive end of $\Sigma$. In this way, we find $\left(\psi_{-}, \psi_{\Sigma}, \psi_{+}\right)$satisfying conditions (i) and (ii) from Step 1. By the $L_{2}^{2}$ bounds on the $\psi$ 's from Lemma 7.7, condition (iii) will also hold if $\delta$ is sufficiently small.

Proof of Lemma 7.5, Step 3. We now show that if $r>r_{1}$ is sufficiently large, then there exists $\delta_{2} \in\left(0, \delta_{1}\right)$ such that if $\delta \leq \delta_{2}$, then the inputs coming from Definition 7.1 can be modified so that the extensions $\psi_{-}, \psi_{\Sigma}, \psi_{+}$ produced by Step 2 also satisfy condition (iv) in Step 1.

To measure the failure of condition (iv), let $\nu_{-}$denote the section of the normal bundle to $u_{-}$( or $u_{-T}$ ) determined by infinitesimal translation of $u_{-}$in the $\mathbb{R}$ direction in $\mathbb{R} \times Y$. Then $\left|\nu_{-}\right| \leq 1$; and since $u_{-}$has index 1 and is unobstructed, $\nu_{-}$spans $\operatorname{Ker}\left(D_{-}\right)$. Define $\nu_{+}$analogously for $u_{+}$ (or $u_{+T}$ ). Then condition (iv) holds if and only if the $L^{2}$ inner products $\left\langle\psi_{-}, \nu_{-}\right\rangle,\left\langle\psi_{+}, \nu_{+}\right\rangle$both vanish.

To understand the inner product $\left\langle\psi_{-}, \nu_{-}\right\rangle$, it proves convenient to write the $L^{2}$ inner product on $u_{-T}$ as $\left\langle\psi_{1}, \psi_{2}\right\rangle=\left\langle\psi_{1}, \psi_{2}\right\rangle_{-}+\left\langle\psi_{1}, \psi_{2}\right\rangle_{+}$, where $\left\langle\psi_{1}, \psi_{2}\right\rangle_{+}$denotes the contribution from the $s \geq s_{-}-T_{-}+r / 2$ portion of $u_{-T}$. By the asymptotic analysis in Section 2 , there are $r$-independent constants $c_{1}, \lambda>0$ such that

$$
\left|\left\langle\psi_{-}, \nu_{-}\right\rangle_{+}\right| \leq c_{1} \delta \exp (-\lambda r) .
$$


By Lemma 7.7, there is a constant $c_{2}$ with

$$
\left|\left\langle\psi_{-}, \nu_{-}\right\rangle_{-}\right| \leq c_{2} \delta .
$$

Now suppose $\delta<\delta_{1}$. Given real numbers $x_{-}, x_{+}$with $\left|x_{ \pm}\right| \leq \delta_{1}-\delta$, consider modifying the data from Definition 7.1 by replacing $\left(R_{-}, R_{+}\right)$with $\left(R_{-}-x_{-}, R_{+}-x_{+}\right)$, while adjusting the sections $\psi_{ \pm}$accordingly so as to describe the same curve $C$. This has the effect of replacing $\left(T_{-}, T_{+}\right)$by $\left(T_{-}+x_{-}, T_{+}-x_{+}\right)$in Step 1. The conditions in Definition 7.1 still hold with $\delta$ replaced by $\delta_{1}$, so we can repeat the procedure in Step 2 to obtain a new triple $\left(\psi_{-}^{x}, \psi_{\Sigma}^{x}, \psi_{+}^{x}\right)$ obeying conditions (i) to (iii) in Step 1.

To compare $\psi_{-}^{x}$ with $\psi_{-}^{0}$, use translation of $s$ to regard both as sections of $u_{-}$. Observe that where $s \leq r / 2$ on $u_{-}$, the image of $e_{-} \circ \psi_{-}^{x}$ is the

$s \mapsto s+x_{-}$translate of the image of $e_{-} \circ \psi_{-}^{0}$. Now the $s \mapsto s+x_{-}$translate of $u_{-}$is the image of $e_{-}\left(x_{-} \nu_{-}+\zeta_{-}\right)$, where $\left|\zeta_{-}\right| \leq c\left|x_{-}\right|^{2}$. It follows that where $s \leq r / 2$ on $u_{-}$, we can write

$$
\psi_{-}^{x}=\psi_{-}^{0}+x_{-} \nu_{-}+\gamma_{-}, \quad\left|\gamma_{-}\right| \leq c\left|x_{-}\right|\left(\left|x_{-}\right|+\delta\right) .
$$

This representation of $\psi_{-}^{x}$ is not valid on all of $u_{-}$. Nonetheless, $\psi_{-}^{x}$ and $\psi_{+}^{x}$ vary smoothly on the whole of $u_{-}$and $u_{+}$as $x=\left(x_{-}, x_{+}\right)$varies in the square $\Omega \subset \mathbb{R}^{2}$ where both coordinates are less than $\delta_{1}-\delta$. This follows from the inverse function theorem, since the map $\mathcal{F}$ that appears in the proof of Lemma 7.7 varies smoothly as $x$ varies.

Now define $f: \Omega \rightarrow \mathbb{R}^{2}$ by sending $x=\left(x_{1}, x_{2}\right) \in \Omega$ to the pair $\left(\left\langle\psi_{-}^{x}, \nu_{-}\right\rangle,\left\langle\psi_{+}^{x}, \nu_{+}\right\rangle\right)$. By the previous paragraph, $f$ is a smooth map. Expanding $\psi_{-}^{x}$ as in (7.12), using the estimate (7.11) for $\psi_{-}^{0}$ and using the estimate (7.10) for $\psi_{-}^{x}$, we find that

$$
\left\langle\psi_{-}^{x}, \nu_{-}\right\rangle=c_{-} x_{-}+\mathfrak{r}_{-},
$$

where $c_{-}$depends only on $u_{-}$and

$$
\left|\mathfrak{r}_{-}\right| \leq c\left(\delta+\exp (-\lambda r)\left|x_{-}\right|+\left|x_{-}\right|^{2}\right) .
$$

Studying $\psi_{+}^{x}$ in the same way, we obtain analogs of (7.13) and (7.14) with the '-' subscripts replaced by ' + ' subscripts. Since $f$ is continuous, a standard topological argument finds a point $x_{0} \in \Omega$ with $f\left(x_{0}\right)=0$, provided that $r$ is sufficiently large and $\delta$ is sufficiently small.

\section{Deforming to the linearized section}

Continue with the gluing setup from Section 5. Recall that Theorem 7.3 relates gluings of $U_{+}$and $U_{-}$along a branched covered cylinder to zeroes of a section $\mathfrak{s}: \times_{2}[5 r, \infty) \times \mathcal{M} \rightarrow \mathcal{O}$. To count the ends of the corresponding index 2 moduli space, we need to count the zeroes of $\mathfrak{s}$ over an appropriate slice of the quotient of the domain by an $\mathbb{R}$-action, as explained below. It is 
difficult to count these zeroes directly because the section $\mathfrak{s}$ involves functions that are defined only implicitly through Proposition 5.7. To facilitate this count, we now explain how to deform $\mathfrak{s}$ to a simpler section, the "linearized section" $\mathfrak{s}_{0}$, without any zeroes crossing the boundary of the relevant slice of the domain, so that the count of zeroes does not change. After defining the linearized section in Section 8.1, we state the deformation result in Section 8.2 and prove it in Section 8.3 to Section 8.5.

8.1. The linearized section $\mathfrak{s}_{0}$. We now define the linearized section $\mathfrak{s}_{0}$. Note that we previously defined a version of the linearized section in Secion I.3, over a slightly different domain; the definition given here is essentially equivalent to the definition given there, as we will explain in Remark 8.5.

Recall the notation $\lambda_{i}$ from Section 5.6. For $i=1, \ldots, \bar{N}_{+}$or $i=$ $-1, \ldots,-\bar{N}_{-}$, let $\mathcal{B}_{i}$ denote the $\lambda_{i}$ eigenspace of $L_{a_{i}}$. Recall from the asymptotic analysis in Proposition 2.4 that there is a constant $\kappa>0$, and for each $i$ as above, there is an eigenfunction $\gamma_{i} \in \mathcal{B}_{i}$ such that for $i=1, \ldots, \bar{N}_{+}$, the function $\eta_{i}$ describing the $i$ th negative end of $u_{+}$satisfies

$$
\left|\eta_{i}(s, \tau)-e^{\left|\lambda_{i}\right| s} \gamma_{i}(\tau)\right| \leq c e^{\left(\left|\lambda_{i}\right|+\kappa\right) s} .
$$

Likewise, for $i=-1, \ldots,-\bar{N}_{-}$, the function $\eta_{i}$ describing the $i$ th positive end of $u_{-}$satisfies

$$
\left|\eta_{i}(s, \tau)-e^{-\lambda_{i} s} \gamma_{i}(\tau)\right| \leq c e^{\left(-\lambda_{i}-\kappa\right) s}
$$

Here, as usual, $c$ denotes a constant that depends only on $u_{+}$and $u_{-}$, but which may change from one appearance to the next.

We will need to assume that the collection of eigenfunctions $\gamma=\left\{\gamma_{i}\right\}$ given by (8.1) and (8.2) is admissible in the sense of Definition I.3.2. This means that the following two conditions hold:

(1) All negative ends of $u_{+}$and all positive ends of $u_{-}$are nondegenerate. That is, $\gamma_{i} \neq 0$ for each $i \in\left\{1, \ldots, \bar{N}_{+}\right\} \cup\left\{-1, \ldots,-\bar{N}_{-}\right\}$.

(2) $u_{+}$does not have a pair of overlapping negative ends, and $u_{-}$does not have a pair of overlapping positive ends. That is, suppose $i, j \in$ $\left\{1, \ldots, \bar{N}_{+}\right\}$satisfy $\left\lceil a_{i} \theta\right\rceil / a_{i}=\left\lceil a_{j} \theta\right\rceil / a_{j}$, or $i, j \in\left\{-1, \ldots,-\bar{N}_{-}\right\}$ satisfy $\left\lfloor a_{i} \theta\right\rfloor / a_{i}=\left\lfloor a_{j} \theta\right\rfloor / a_{j}$, so that the eigenspaces $\mathcal{B}_{i}$ and $\mathcal{B}_{j}$ are identified via coverings. Then for all $g_{i} \in \mathbb{Z} / a_{i}$ and $g_{j} \in \mathbb{Z} / a_{j}$, the action by deck transformations satifies $g_{i} \cdot \gamma_{i} \neq g_{j} \cdot \gamma_{j}$.

Propositions 3.2 and 3.9 guarantee that $\gamma$ is admissible if $J$ is generic.

Now fix a branched cover $\Sigma \in \mathcal{M}$, and let $\sigma \in \operatorname{Coker}\left(D_{\Sigma}\right)$. Recall from Section I.2.3 that the metric on each $\Sigma \in \mathcal{M}$ is chosen to agree with the pullback of the metric on $\mathbb{R} \times S^{1}$, except on neighborhoods of the ramification points that project to balls of radius 1 in $\mathbb{R} \times S^{1}$. Let $D_{\Sigma}^{*}$ denote the formal adjoint of $D_{\Sigma}$ with respect to this metric, and identify $\sigma$ with an element 
of $\operatorname{Ker}\left(D_{\Sigma}^{*}\right)$. On the complement of the ramification points in $\Sigma$, use $d \bar{z}$ to trivialize $T^{0,1} \Sigma$, and thereby regard $\sigma$ as a complex-valued function. On the complement of the aforementioned neighborhoods of the ramification points, $\sigma$ satisfies the equation

$$
\left(\partial_{s}-i \partial_{t}-S(t)\right) \sigma=0
$$

where $S(t)$ is a symmetric $2 \times 2$ real matrix, see Section I.2.2. For $i=$ $1, \ldots, \bar{N}_{+}$, the restriction of $\sigma$ to the $i$ th positive end of $\Sigma$ determines a function $\sigma_{i}:\left[s_{i}, \infty\right) \times \widetilde{S^{1}} \rightarrow \mathbb{C}$, where $\widetilde{S^{1}}$ denotes the $a_{i}$-fold cover of $S^{1}$. Likewise, for $i=-1, \ldots,-\bar{N}_{-}$, the restriction of $\sigma$ to the $i$ th negative end of $\Sigma$ determines a function $\sigma_{i}:\left(-\infty, s_{i}\right] \times \widetilde{S^{1}} \rightarrow \mathbb{C}$. In either case, equation (8.3) on the end becomes

$$
\left(\partial_{s}-L_{a_{i}}\right) \sigma_{i}(s, \cdot)=0
$$

Let $\Pi_{\mathcal{B}}$ denote the projection in $L^{2}\left(\widetilde{S^{1}} ; \mathbb{R}^{2}\right)$ to $\mathcal{B}_{i}$. Then it follows from (8.4) that there is an eigenfunction $\beta_{i} \in \mathcal{B}_{i}$ such that

$$
\Pi_{\mathcal{B}} \sigma_{i}(s, \cdot)=e^{\lambda_{i}\left(s-s_{i}\right)} \beta_{i} .
$$

Definition 8.1. Define the linearized section $\mathfrak{s}_{0}: \times_{2}(5 r, \infty) \times \mathcal{M} \rightarrow \mathcal{O}$ as follows. If $\sigma \in \operatorname{Coker}\left(D_{\Sigma}\right)$ has associated eigenfunctions $\beta_{i} \in \mathcal{B}_{i}$, then

$$
\mathfrak{s}_{0}\left(T_{-}, T_{+}, \Sigma\right)(\sigma):=\sum_{i=1}^{\bar{N}_{+}} e^{-\nu_{i}}\left\langle\gamma_{i}, \beta_{i}\right\rangle-\sum_{i=-1}^{-\bar{N}_{-}} e^{-\nu_{i}}\left\langle\gamma_{i}, \beta_{i}\right\rangle,
$$

where the brackets denote the inner product on $L^{2}\left(\widetilde{S^{1}}, \mathbb{R}^{2}\right)$, and

$$
\nu_{i}:= \begin{cases}\left|\lambda_{i}\right|\left(s_{+}-s_{i}+T_{+}\right), & i=1, \ldots, \bar{N}_{+}, \\ \lambda_{i}\left(s_{i}-s_{-}+T_{-}\right), & i=-1, \ldots,-\bar{N}_{-} .\end{cases}
$$

The linearized section $\mathfrak{s}_{0}$ appears as part of the original section $\mathfrak{s}$, as follows. By equation (5.43), we can write

$$
\mathfrak{s}\left(T_{-}, T_{+}, \Sigma\right)(\sigma)=\left\langle\sigma, \eta^{\prime}+\mathcal{R}\left(\psi_{\Sigma}\right)\right\rangle
$$

where

$$
\eta^{\prime}:=\frac{1}{2}\left(\frac{\partial \beta_{-}}{\partial s} \eta_{-T}+\frac{\partial \beta_{+}}{\partial s} \eta_{+T}\right) d \bar{z}
$$

while $\mathcal{R}\left(\psi_{\Sigma}\right)$ denotes the sum of all the other terms in (5.13) that enter into $\mathcal{F}_{\Sigma}\left(\psi_{\Sigma}\right)$. Recall that $\eta^{\prime}$ is supported on the ends of $\Sigma$ labeled by $1, \ldots, \bar{N}_{+}$ and $-1, \ldots,-\bar{N}_{-}$. Let $\Pi_{\mathcal{B}} \eta^{\prime}$ denote the $(0,1)$-form on $\Sigma$ obtained from $\eta^{\prime}$ by projecting, for each $i$, the part of $\eta^{\prime}$ on the $i$ th end onto the eigenspace $\mathcal{B}_{i}$. Then equation (8.6) can be rewritten as

$$
\mathfrak{s}_{0}\left(T_{-}, T_{+}, \Sigma\right)(\sigma)=\sqrt{2}\left\langle\sigma, \Pi_{\mathcal{B}} \eta^{\prime}\right\rangle .
$$


8.2. Counting zeroes of the obstruction section and its linearization. Given $R \geq 10 r$, let $\mathcal{V}_{R}$ denote the set of triples $\left(T_{-}, T_{+}, \Sigma\right) \in$ $\times_{2}[5 r, \infty) \times \mathcal{M}$ such that

$$
T_{+}+s_{+}-s_{-}+T_{-}=R
$$

This means that the curves $U_{+}$and $U_{-}$are translated away from each other by distance $R$ in the pregluing. We will see in Section 10 that the signed count of gluings $\# G\left(u_{+}, u_{-}\right)$is determined by a count of zeroes of $\mathfrak{s}$ on $\mathcal{V}_{R}$, modulo a certain $\mathbb{R}$-action, where $R$ is fixed and large. We now want to show that counting zeroes of $\mathfrak{s}_{0}$ will give the same result.

For this purpose we will linearly interpolate from $\mathfrak{s}$ to $\mathfrak{s}_{0}$. For each $t \in[0,1]$ define a section

$$
\mathfrak{s}_{t}:=t \mathfrak{s}+(1-t) \mathfrak{s}_{0} .
$$

The following proposition implies that $\mathfrak{s}_{t}$ has no zeroes on the boundary of $\mathcal{V}_{R}$ when $R$ is fixed and large. To state it, let $\lambda$ denote the smallest of the numbers $\left|\lambda_{i}\right|$ for $i=1, \ldots, \bar{N}_{+}$and $i=-1, \ldots,-\bar{N}_{-}$, and let $\Lambda$ denote the largest of these numbers.

Proposition 8.2. Assume that $\gamma$ is admissible as in Section 8.1. In the gluing construction, if we choose $r$ sufficiently large and $h<\lambda / 4 \Lambda$, then for all $t \in[0,1]$, every triple $\left(T_{-}, T_{+}, \Sigma\right) \in \times_{2}[5 r, \infty) \times \mathcal{M}$ with $\mathfrak{s}_{t}\left(T_{-}, T_{+}, \Sigma\right)=0$ satisfies

$$
T_{+}, T_{-}>\frac{\lambda R}{3 \Lambda}
$$

where $R$ is defined by (8.10).

To relate this to counting zeroes, first recall that $\mathbb{R}$ acts on the moduli space of branched covers $\mathcal{M}$ by translating the $s$ coordinate. We extend this to an action on $\times_{2}[5 r, \infty) \times \mathcal{M}$ fixing the $[5 r, \infty)$ factors. This $\mathbb{R}$ action extends to the obstruction bundle. That is, if $\Sigma_{1}, \Sigma_{2} \in \mathcal{M}$ are in the same orbit under the $\mathbb{R}$ action, then there is a canonical isomorphism $\operatorname{Coker}\left(D_{\Sigma_{1}}\right)=\operatorname{Coker}\left(D_{\Sigma_{2}}\right)$. It follows directly from the definitions that under the above identification,

$$
\mathfrak{s}_{t}\left(T_{-}, T_{+}, \Sigma_{1}\right)=\mathfrak{s}_{t}\left(T_{-}, T_{+}, \Sigma_{2}\right)
$$

for each $t \in[0,1]$. Thus $\mathfrak{s}_{t}$ is well defined on $\times_{2}[5 r, \infty) \times \mathcal{M} / \mathbb{R}$.

We now want to count zeroes of $\mathfrak{s}_{t}$ over $\mathcal{V}_{R} / \mathbb{R}$, where $R$ is fixed and large. For this purpose, note that there is a natural identification

$$
\mathcal{V}_{R} / \mathbb{R} \simeq\left\{\Sigma \in \mathcal{M} \mid-R / 2+5 r \leq s_{-}, s_{+} \leq R / 2-5 r\right\} .
$$

Given a branched cover $\Sigma$ for which $-R / 2+5 r \leq s_{-}$and $s_{+} \leq R / 2-5 r$, this identification sends

$$
\Sigma \longmapsto\left(s_{-}+R / 2, R / 2-s_{+},[\Sigma]\right) \in \mathcal{V}_{R} / \mathbb{R}
$$


Since $\mathcal{M}$ is a smooth manifold, it follows that the interior of $\mathcal{V}_{R} / \mathbb{R}$ is a smooth manifold, and the boundary $\partial\left(\mathcal{V}_{R} / \mathbb{R}\right)$ is identified with the set of branched covers $\Sigma$ for which $s_{-}=-R / 2+5 r$ or $s_{+}=R / 2-5 r$. Such branched covers correspond to equivalence classes of triples $\left(T_{-}, T_{+}, \Sigma\right) \in \mathcal{V}_{R}$ with $T_{-}=5 r$ or $T_{+}=5 r$. Moreover, since $\mathcal{M}$ has a canonical orientation as a complex manifold, the identification (8.11) defines an orientation of $\operatorname{int}\left(\mathcal{V}_{R} / \mathbb{R}\right)$.

Definition 8.3. Assume that $\gamma$ is admissible. Fix $h<\lambda / 4 \Lambda$ and $r \gg 0$ in the gluing construction. Given $R>15 r \Lambda / \lambda$ and $t \in[0,1]$, define the relative Euler class

$$
e\left(\mathcal{O} \longrightarrow \mathcal{V}_{R} / \mathbb{R}, \mathfrak{s}_{t}\right) \in \mathbb{Z}
$$

as follows: Let $\mathfrak{s}_{t}^{\prime}$ be a section of $\mathcal{O}$ over $\mathcal{V}_{R} / \mathbb{R}$ such that $\mathfrak{s}_{t}^{\prime}=\mathfrak{s}_{t}$ on $\partial\left(\mathcal{V}_{R} / \mathbb{R}\right)$, and such that all zeroes of $\mathfrak{s}_{t}^{\prime}$ are nondegenerate. Define $e\left(\mathcal{O} \rightarrow \mathcal{V}_{R} / \mathbb{R}, \mathfrak{s}_{t}\right)$ to be the signed count of zeroes of $\mathfrak{s}_{t}^{\prime}$, using the orientation of $\mathcal{V}_{R} / \mathbb{R}$ determined by (8.11) and the orientation of $\mathcal{O}$ defined in Section I.2.6. We usually denote this count by $\#\left(\mathfrak{s}_{t}^{-1}(0) \cap \mathcal{V}_{R} / \mathbb{R}\right)$, even though the zeroes of $\mathfrak{s}_{t}$ itself on $\mathcal{V}_{R} / \mathbb{R}$ may be degenerate.

Lemma 8.4. The relative Euler class $\#\left(\mathfrak{s}_{t}^{-1}(0) \cap \mathcal{V}_{R} / \mathbb{R}\right)$ is well defined and does not depend on the choice of $R>15 \mathrm{r} \Lambda / \lambda$ or $t \in[0,1]$.

Proof. We know from Proposition 6.1 that the family of sections $\mathfrak{s}_{t}$ is continuous. Furthermore, Proposition 8.2 guarantees that $\mathfrak{s}_{t}$ is nonvanishing near $\partial\left(\mathcal{V}_{R} / \mathbb{R}\right)$. Hence the only issue is to check that $\mathcal{V}_{R} / \mathbb{R}$ is compact.

For this purpose, the key is to show that

$\left(^{*}\right)$ for any $\Sigma \in \mathcal{M}$, all ramification points have $\pi^{*} s \in\left[s_{-}, s_{+}\right]$.

To prove $\left(^{*}\right)$, recall that since $\left(U_{+}, U_{-}\right)$is a gluing pair, it is required that under the partial order $\geq_{\theta}$ in Definition I.1.8, the partition $\left(a_{\bar{N}_{+}+1}, \ldots, a_{N_{+}}\right)$ is minimal and the partition $\left(a_{-\bar{N}_{-}-1}, \ldots, a_{-N_{-}}\right)$is maximal. Now suppose that $\Sigma \in \mathcal{M}$ has a ramification point with $\pi^{*} s>s_{+}$. Then we can decompose $\Sigma=\Sigma_{1} \sqcup \Sigma_{2}$, where $\Sigma_{1}$ contains this ramification point and has positive ends indexed by $\bar{N}_{+}+1, \ldots, N_{+}$. Our standing assumption (1.2) implies that $\Sigma$ has index zero, and hence so do $\Sigma_{1}$ and $\Sigma_{2}$, see Section I.1.2. The existence of $\Sigma_{1}$ directly contradicts the minimality of the partition $\left(a_{N_{+}+1}, \ldots, a_{N_{+}}\right)$. Likewise, maximality of the partition $\left(a_{-} \bar{N}_{-}, 1, \ldots, a_{-N_{-}}\right)$forbids the existence of a ramification point with $\pi^{*} s<s_{-}$.

It follows from $\left(^{*}\right)$ that (8.11) identifies $\mathcal{V}_{R} / \mathbb{R}$ with a subset of $\mathcal{M}_{R / 2-5 r}$. Now $\mathcal{M}_{R / 2-5 r}$ is compact by the assumption (1.2) and Lemma I.2.8. Hence, any sequence in $\mathcal{V}_{R} / \mathbb{R}$ has a subsequence whose corresponding branched covers converge to some element of $\mathcal{M}_{R / 2-5 r}$. By continuity of the functions $s_{+}$and $s_{-}$, this limiting branched cover corresponds to an element of $\mathcal{V}_{R} / \mathbb{R}$. 
Remark 8.5. To coordinate the counting here with that in Part I, we need to compare the formalism here with that of Section I.3. In Section I.3.1, given $R, r>0$ and given admissible eigenfunctions $\gamma$, we defined $\mathfrak{s}_{0}$ as a section over $\mathcal{M}_{R}$. By $\left(^{*}\right)$ above, (8.11) identifies $\mathcal{V}_{R} / \mathbb{R}$ with a subset of $\mathcal{M}_{R / 2-5 r}$ that contains all of $\mathcal{M}_{R / 2-5 r-1}$. Under this identification, the definition of $\mathfrak{s}_{0}$ over $\mathcal{V}_{R} / \mathbb{R}$ given here is a special case of the definition in Section I.3.1, where $(R, r)$ here corresponds to $(R / 2-5 r, 5 r)$ there, and where we take $\gamma$ to be the eigenfunctions determined by the negative ends of $u_{+}$and the positive ends of $u_{-}$. In particular, the $R$-independent count $\#\left(\mathfrak{s}_{0}^{-1}(0) \cap\right.$ $\left.\mathcal{V}_{R}\right) / \mathbb{R}$ ) defined above agrees with the corresponding count $\# \mathfrak{s}_{0}^{-1}(0)$ defined in Section I.3.2. The reason is that Proposition 8.2 implies that $\mathfrak{s}_{0}$ has no zeroes on $\mathcal{M}_{R / 2-5 r} \backslash \mathcal{M}_{R / 2-5 r-1}$ if $R \gg r \gg 0$.

In conclusion, we have:

Corollary 8.6. Assume that $J$ is generic so that $\gamma$ is admissible. If $r$ is chosen sufficiently large and if $h$ is chosen sufficiently small in the gluing construction, then for $R$ sufficiently large, the relative Euler class $\#\left(\mathfrak{s}^{-1}(0) \cap\right.$ $\left.\mathcal{V}_{R} / \mathbb{R}\right)$ is well-defined, does not depend on $R$ and satisfies

$$
\#\left(\mathfrak{s}^{-1}(0) \cap \mathcal{V}_{R} / \mathbb{R}\right)=\#\left(\mathfrak{s}_{0}^{-1}(0) \cap \mathcal{V}_{R} / \mathbb{R}\right)
$$

In Section 10 we will relate the the left hand side of (8.12) to the signed count of gluings $\# G\left(u_{+}, u_{-}\right)$. The rest of Section 8 is devoted to the proof of Proposition 8.2, beginning with some preliminary lemmas.

8.3. Nonlinear estimate. The proof of Proposition 8.2 will use an upper bound on the term $\left\langle\sigma, \mathcal{R}\left(\psi_{\Sigma}\right)\right\rangle$ in equation (8.8). To state this bound, let $\nu$ denote the smallest of the numbers $\nu_{i}$ defined in (8.7). Also recall the norms $\|\cdot\|$ and $\|\cdot\|_{*}$ from Section 5.5.

Lemma 8.7. Suppose that in the gluing construction, $h$ is chosen so that $4 h \Lambda<\lambda$. Then

$$
\left\|\mathcal{R}\left(\psi_{\Sigma}\right)\right\| \leq c e^{-\nu-\lambda r / 2}
$$

Proof. Estimating $\left\|\mathcal{R}\left(\psi_{\Sigma}\right)\right\|$ as in (5.42), we find that

$$
\left\|\mathcal{R}\left(\psi_{\Sigma}\right)\right\| \leq c\left(\left\|\psi_{\Sigma}\right\|_{*}^{2}+e^{-\lambda r}\left\|\psi_{\Sigma}\right\|_{*}\right)
$$

Note that the term $e^{-\lambda r}\left\|\psi_{\Sigma}\right\|_{*}$ appears here because of the $\mathfrak{q}_{0} \cdot \psi_{\Sigma}$ and $\mathfrak{q}_{0}^{\prime} \cdot \nabla \psi_{\Sigma}$ terms in $\mathcal{R}\left(\psi_{\Sigma}\right)$. 
Using Proposition 5.7(b) and our assumption that $4 h \Lambda<\lambda$, we estimate

$$
\begin{aligned}
\left\|\psi_{\Sigma}\right\|_{*} & \leq c\left(\sum_{i=1}^{\bar{N}_{+}} e^{-\left|\lambda_{i}\right|\left(s_{+}-s_{i}+T_{+}-2 r h\right)}+\sum_{i=-1}^{-\bar{N}_{-}} e^{-\lambda_{i}\left(s_{i}-s_{-}+T_{-}-2 r h\right)}\right) \\
& =c\left(\sum_{i=1}^{\bar{N}_{+}} e^{-\nu_{i}+2 r h\left|\lambda_{i}\right|}+\sum_{i=-1}^{-\bar{N}_{-}} e^{-\nu_{i}+2 r h \lambda_{i}}\right) \\
& \leq c e^{-\nu+\lambda r / 2} .
\end{aligned}
$$

Putting (8.15) into (8.14) gives

$$
\left\|\mathcal{R}\left(\psi_{\Sigma}\right)\right\| \leq c\left(e^{-2 \nu+\lambda r}+e^{-\nu-\lambda r / 2}\right) .
$$

It follows from the definitions that $\nu \geq 5 \lambda r$. Hence (8.16) implies (8.13).

8.4. Ends with the same eigenvalue. The proof of Proposition 8.2 will also need Lemma 8.8 below, regarding the structure of the cokernel in the case when $N_{-}=1$ and the eigenvalues $\lambda_{1}, \ldots, \lambda_{N_{+}}$are all equal, say to $\lambda$. By Remark I.2.12, this last condition is equivalent to

$$
\frac{\left\lceil a_{1} \theta\right\rceil}{a_{1}}=\cdots=\frac{\left\lceil a_{N_{+}} \theta\right\rceil}{a_{N_{+}}} .
$$

Recall from Section I.3.1 that the assumption (8.17) allows us to identify all the eigenspaces $\mathcal{B}_{1}, \ldots, \mathcal{B}_{N_{+}}$with each other via coverings as follows. Write $\left\lceil a_{1} \theta\right\rceil / a_{1}=\eta_{0} / m_{0}$ where $\eta_{0}$ and $m_{0}$ are integers and $m_{0}>0$ is as small as possible. Then $a_{i}$ is divisible by $m_{0}$ for each $i=1, \ldots, N_{+}$. Fix an eigenfunction $\varphi_{m_{0}}$ of $L_{m_{0}}$ with eigenvalue $\lambda$. Then for every positive integer $d$, the eigenfunction $\varphi_{m_{0}}$ pulls back to an eigenfunction $\varphi_{d m_{0}}$ of $L_{d m_{0}}$ with the same eigenvalue $\lambda$. There is now a canonical isomorphism from $\mathcal{B}_{i}$ to $\mathcal{B}_{j}$ sending $\varphi_{a_{i}}$ to $\varphi_{a_{j}}$. This identification is made implicitly below.

Also note that the product of cyclic groups

$$
G:=\mathbb{Z} / a_{1} \times \cdots \times \mathbb{Z} / a_{N_{+}}
$$

acts on $\bigoplus_{i=1}^{N_{+}} \mathcal{B}_{i}$. Here the $i$ th factor $\mathbb{Z} / a_{i}$ acts on $\mathcal{B}_{i}$ by deck transformations of the corresponding eigenfunctions, and trivially on $\mathcal{B}_{j}$ for $j \neq i$.

Now given $\Sigma \in \mathcal{M}$, define $\Pi^{+}: \operatorname{Coker}\left(D_{\Sigma}\right) \rightarrow \bigoplus_{i=1}^{N_{+}} \mathcal{B}_{i}$ as follows. Given $\sigma \in \operatorname{Coker}\left(D_{\Sigma}\right)$, for $i=1, \ldots, N_{+}$, write $\Pi_{\mathcal{B}} \sigma_{i}(s, \cdot)=e^{\lambda s} \zeta_{i}$; then

$$
\Pi^{+}(\sigma):=\left(\zeta_{1}, \ldots, \zeta_{N_{+}}\right) \text {. }
$$

Lemma 8.8. Suppose that $N_{-}=1$ and that (8.17) holds. Then for any $\Sigma \in \mathcal{M}$, there exists $g \in G$ such that

$$
g \cdot \Pi^{+}\left(\operatorname{Coker}\left(D_{\Sigma}\right)\right) \subset\left\{\left(\zeta_{1}, \ldots, \zeta_{N_{+}}\right) \mid \sum_{i=1}^{N_{+}} \zeta_{i}=0\right\} .
$$


Proof. Assume below that the eigenvalue $\lambda$ of $L_{a_{1}}, \ldots, L_{a_{N_{+}}}$is not repeated; the proof when $\lambda$ is repeated is similar.

Let $\Sigma \in \mathcal{M}$. Recall from Section I.2.1 that $\Sigma$ determines an oriented weighted tree $\tau(\Sigma)$ whose edges correspond to cylinders in $\Sigma$ between ramification points. By downward induction, for each edge $e$ of the tree $\tau(\Sigma)$, the covering multiplicity $m(e)$ of the corresponding cylinder in $\Sigma$ is divisible by $m_{0}$. Hence, we can lift the branched covering $\pi$ on $\Sigma$ to a continuous map

$$
\tilde{\pi}: \Sigma \longrightarrow \mathbb{R} \times \mathbb{R} / 2 \pi m_{0} \mathbb{Z} .
$$

For each $i=1, \ldots, N_{+}$, choose an identification of the $i$ th positive end of $\Sigma$ with $\left[s_{i}, \infty\right) \times \mathbb{R} / 2 \pi a_{i} \mathbb{Z}$, such that the projection to $\left[s_{i}, \infty\right) \times \mathbb{R} / 2 \pi m_{0} \mathbb{Z}$ agrees with $\widetilde{\pi}$. This identification will differ from the asymptotic marking by the action of some $g_{i} \in \mathbb{Z} / a_{i}$. Let $g:=\left(g_{1}, \ldots, g_{N_{+}}\right)$.

Next, let $\sigma \in \operatorname{Coker}\left(D_{\Sigma}\right)$, and write $g \cdot \Pi^{+} \sigma=\left(\zeta_{1}, \ldots, \zeta_{N_{+}}\right)$. Fix a smooth function $\chi: \mathbb{R} \rightarrow[0,1]$ such that $\chi(s)=1$ when $s \leq s_{+}+2$ and $\chi(s)=0$ when $s \geq s_{+}+3$. Write $\widetilde{\pi}=(s, \widetilde{t})$. Define a function $f: \Sigma \rightarrow \mathbb{C}$ by

$$
f:=\chi(s) e^{-\lambda s} \varphi_{m_{0}}(\widetilde{t})
$$

Recall that on any cylinder in $\Sigma$ corresponding to an edge $e$ of $\tau(\Sigma)$, the operator $D_{\Sigma}$ has the form $\frac{1}{2} d \bar{z} \otimes\left(\partial_{s}+L_{m(e)}\right)$. It follows that

$$
D_{\Sigma} f=\frac{1}{2}\left(\partial_{s} \chi\right) e^{-\lambda s} \varphi_{m_{0}}(\widetilde{t}) d \bar{z} .
$$

In particular, $D_{\Sigma} f$ is supported only where $s_{+}+2 \leq s \leq s_{+}+3$, and here the metric on $\Sigma$ agrees with the pullback of the metric on $\mathbb{R} \times S^{1}$. Also, $f$ is $L_{1}^{2}$ since $\lambda<0$. We then have

$$
0=\left\langle D_{\Sigma}^{*} \sigma, f\right\rangle=\left\langle\sigma, D_{\Sigma} f\right\rangle=\sum_{i=1}^{N_{+}}\left\langle\zeta_{i}, \varphi_{a_{i}}\right\rangle
$$

where the brackets denote the relevant $L^{2}$ inner products. Under our identifications of the $\mathcal{B}_{i}$ 's, this means that $\sum_{i=1}^{N_{+}} \zeta_{i}=0$.

It is not hard to further show, using Lemma I.2.18(a), that the inclusion in (8.18) is actually an equality. However, we will not need this.

8.5. Proof of the deformation result. With the preliminaries in place, we now prove Proposition 8.2. Fix $h<\lambda / 4 \Lambda$ and suppose that Proposition 8.2 is false for this $h$. Then:

(*) For each $n=1,2, \ldots$ there exist real numbers $r_{n} \geq n$ and $t_{n} \in[0,1]$, and a triple $\left(T_{-n}, T_{+n}, \Sigma_{n}\right) \in \times_{2}\left[5 r_{n}, \infty\right) \times \mathcal{M}$, such that if we fix $r=r_{n}$ in the gluing construction, then $\mathfrak{s}_{t_{n}}\left(T_{-n}, T_{+n}, \Sigma_{n}\right)=0$ and $\min \left\{T_{-n}, T_{+n}\right\} \leq \lambda R_{n} / 3 \Lambda$. 
Here $R_{n}:=T_{+n}+s_{+n}-s_{-n}+T_{-n}$, where $s_{ \pm n}$ denotes the value of $s_{ \pm}$for $\Sigma_{n}$. We will use $(*)$ to deduce a contradiction, in four steps.

Step 1. We begin with some setup. Recall that associated to each $\Sigma_{n}$ is a tree $\tau\left(\Sigma_{n}\right)$, with a projection $p: \Sigma_{n} \rightarrow \tau\left(\Sigma_{n}\right)$ and a metric coming from the $s$ coordinate. By passing to a subsequence, we may assume that the sequence of branched covers $\left\{\Sigma_{n}\right\}$ in $\mathcal{M} / \mathbb{R}$ converges in the sense of Definition I.2.27 to a tree $\tau_{*}$ together with a branched cover $\Sigma_{* j}$ for each internal vertex $j$ of $\tau_{*}$. Note that conditions (a) to (d) in Definition I.2.27 imply that:

- For each $\Sigma_{n}$ in the sequence and for each internal vertex $j$ of $\tau_{*}$, there is a corresponding set $\Lambda_{n j}$ of ramification points in $\Sigma_{n}$. The tree $\tau_{*}$ is obtained from the tree $\tau\left(\Sigma_{n}\right)$ by, for each $j$, collapsing all the vertices in $\tau\left(\Sigma_{n}\right)$ corresponding to ramification points in $\Lambda_{n j}$ and all the edges between them to the $j$ th vertex of $\tau_{*}$.

- There is an $n$-independent constant $\Delta_{*}$ such that any two ramification points in the same $\Lambda_{n j}$ project to points in the tree $\tau\left(\Sigma_{n}\right)$ with distance $\leq \Delta_{*}$.

- If $j$ and $j^{\prime}$ are distinct internal vertices of $\tau_{*}$, then for each $n$, in the tree $\tau\left(\Sigma_{n}\right)$ we have $\lim _{n \rightarrow \infty} \operatorname{dist}\left(p\left(\Lambda_{n j}\right), p\left(\Lambda_{n j^{\prime}}\right)\right)=\infty$.

By passing to a further subsequence, we may improve this last condition to

$$
\operatorname{dist}\left(p\left(\Lambda_{n j}\right), p\left(\Lambda_{n j^{\prime}}\right)\right) \geq n .
$$

Now fix $n$ large and drop the " $n$ " subscripts below. Choose $i_{1}$ such that $\nu_{i_{1}}=\nu$ for $\Sigma=\Sigma_{n}$. Without loss of generality, $i_{1} \in\left\{1, \ldots, \bar{N}_{+}\right\}$. Let $j$ denote the internal vertex of $\tau_{*}$ that is adjacent to the leaf $i_{1}$. If $e$ is an edge of $\tau_{*}$ incident to $j$, call $e$ "essential" if $e$ is incident to a leaf $i$ with $\lambda_{i}=\lambda_{i_{1}}$. In particular, this requires that $i$ is positive. If $e$ is an edge of $\tau_{*}$ incident to $j$ which is either internal or incident to a leaf $i$ with $\lambda_{i} \neq \lambda_{i_{1}}$, call $e$ "inessential".

Step 2. We claim that there is an $n$-independent constant $\kappa>0$ such that if there is an inessential edge from the vertex $j$ to the leaf $i$, then $\nu_{i} \geq \nu+\kappa n$ when $n$ is large.

To prove this when $i$ is positive, we compute that

$$
\nu_{i}-\nu=\left(\left|\lambda_{i}\right|-\left|\lambda_{i_{1}}\right|\right)\left(s_{+}-s_{i_{1}}+T_{+}\right)+\left|\lambda_{i}\right|\left(s_{i_{1}}-s_{i}\right),
$$

and observe that $s_{+}-s_{i_{1}}+T_{+} \geq 5 n$ and $\left|s_{i_{1}}-s_{i}\right| \leq \Delta_{*}$. Note that $\left|\lambda_{i}\right|<\left|\lambda_{i_{1}}\right|$ is impossible when $n$ is large, because then (8.19) would imply that $\nu_{i}<\nu$, contradicting the definition of $\nu$. Since the edge from $j$ to $i$ is essential, the only remaining possibility is that $\left|\lambda_{i}\right|>\left|\lambda_{i_{1}}\right|$. The claim now follows immediately from (8.19).

Suppose next that $i$ is negative. It follows from the definitions that

$$
R=\frac{\nu}{\left|\lambda_{i_{1}}\right|}+\frac{\nu_{i}}{\lambda_{i}}+\left(s_{i_{1}}-s_{i}\right) \leq \frac{\nu+\nu_{i}}{\lambda}+\Delta_{*}
$$


and so

$$
\nu_{i} \geq \lambda R-\nu-\lambda \Delta_{*}
$$

Now $T_{+} \geq \nu / \Lambda$, because there exists a positive end $i^{\prime}$ with $s_{i^{\prime}}=s_{+}$. Likewise $T_{-} \geq \nu / \Lambda$. The assumption in (*) that $\min \left\{T_{-}, T_{+}\right\} \leq \lambda R / 3 \Lambda$ then implies that $\lambda R \geq 3 \nu$. Putting this into (8.20) and using the fact that $\nu \geq 5 \lambda n$ proves the claim.

Step 3. We now complete the proof of Proposition 8.2 in the case when there are at least two inessential edges incident to $j$.

Choose paths in $\tau_{*}$ starting along these edges to leaves $i_{2}, i_{3}$. Let $w$ denote the central vertex in the tree $\tau(\Sigma)$ for $i_{1}, i_{2}$ and $i_{3}$. The vertex $w$ in $\tau(\Sigma)$ projects to the vertex $j$ in $\tau_{*}$.

By Lemma I.2.18, there is a unique, nonvanishing $\sigma \in \operatorname{Coker}\left(D_{\Sigma}\right)$ with

$$
\Pi_{\mathcal{A}} \sigma_{i_{1}}(s, \cdot)=e^{\lambda_{i_{1}}\left(s-s_{i_{1}}\right)} \gamma_{i_{1}}
$$

and $\Pi_{\mathcal{A}} \sigma_{i}=0$ for $i \notin\left\{i_{1}, i_{2}, i_{3}\right\}$. Here $\Pi_{\mathcal{A}} \sigma_{i}$ denotes the projection of $\sigma_{i}$ onto the subspace $\mathcal{A}_{i} \supset \mathcal{B}_{i}$ consisting of the (two-dimensional) span of the eigenfunctions of $L_{a_{i}}$ that have the same winding number as the eigenfunctions with eigenvalue $\lambda_{i}$.

For each leaf $i$, let $\beta_{i}$ denote the corresponding eigenfunction associated to $\sigma$ via (8.5). In particular $\beta_{i_{1}}=\gamma_{i_{1}}$. The plan is to show that if $n$ is large, then $\mathfrak{s}_{t}\left(T_{-}, T_{+}, \Sigma\right)(\sigma)$ is dominated by the term $e^{-\nu_{i_{1}}}\left\langle\gamma_{i_{1}}, \beta_{i_{1}}\right\rangle$, and in particular nonzero. This will give the desired contradiction to $\left(^{*}\right)$.

To start, we claim that the special cokernel element $\sigma$ decays away from the central vertex $w$ in the following sense: There are $n$-independent constants $c, \kappa>0$ such that for any point $x \in \Sigma=\Sigma_{n}$,

$$
|\sigma(x)|<c e^{-\kappa \cdot \operatorname{dist}(p(x), w)} .
$$

Indeed, Corollary I.2.23 gives (8.21) with the right hand side multiplied by $|\sigma(\widetilde{w})|$, where $\widetilde{w} \in \Sigma$ projects to $w$; and Propositions I.2.21 and I.2.25 imply that $|\sigma(\widetilde{w})|<c$.

The inequality (8.21) has two important consequences. First,

$$
\|\sigma\|_{L^{2}}<c .
$$

Second, if a leaf $i \in\left\{i_{2}, i_{3}\right\}$ is not adjacent to the internal vertex $j$ of $\tau_{*}$, then

$$
\left\|\beta_{i}\right\| \leq\left\|\Pi_{\mathcal{A}} \sigma_{i}\left(s_{i}, \cdot\right)\right\|<c e^{-\kappa n} .
$$

Likewise, if $i \in\left\{i_{2}, i_{3}\right\}$ is adjacent to $j$, then

$$
\left\|\beta_{i}\right\|<c
$$


Now to show that $\mathfrak{s}_{t}\left(T_{-}, T_{+}, \Sigma\right)(\sigma)$ is nonzero, use (8.8) and (8.9) to write the latter as a sum of three terms:

$$
\begin{aligned}
\mathfrak{s}_{t}\left(T_{-}, T_{+}, \Sigma\right)(\sigma)= & (1+t(\sqrt{2}-1)) \mathfrak{s}_{0}\left(T_{-}, T_{+}, \Sigma\right)(\sigma) \\
& +t\left\langle\sigma, \mathcal{R}\left(\psi_{\Sigma}\right)\right\rangle+t\left\langle\sigma, \eta^{\prime}-\Pi_{\mathcal{B}} \eta^{\prime}\right\rangle
\end{aligned}
$$

Our choice of $\sigma$ implies that the first term in (8.25) is given by

$$
\mathfrak{s}_{0}\left(T_{-}, T_{+}, \Sigma\right)(\sigma)=e^{-\nu}\left\|\gamma_{i_{1}}\right\|^{2} \pm e^{-\nu_{i_{2}}}\left\langle\gamma_{i_{2}}, \beta_{i_{2}}\right\rangle \pm e^{-\nu_{i_{3}}}\left\langle\gamma_{i_{3}}, \beta_{i_{3}}\right\rangle .
$$

Also observe that if $i \in\left\{i_{2}, i_{3}\right\}$, then

$$
\left|e^{-\nu_{i}}\left\langle\gamma_{i}, \beta_{i}\right\rangle\right|<c e^{-\nu-\kappa n} \text {. }
$$

If the leaf $i$ is adjacent to the vertex $j$ in $\tau_{*}$, then this follows from (8.24) and Step 2; otherwise this follows from (8.23). Next, the inequality (8.22) and Lemma 8.7, together with the fact that $r=r_{n} \geq n$, imply that

$$
\left|\left\langle\sigma, \mathcal{R}\left(\psi_{\Sigma}\right)\right\rangle\right| \leq c e^{-\nu-\lambda n / 2} .
$$

Finally, Proposition I.2.25 and the inequality (8.21), together with the fact that $r \geq n$, imply that if $\kappa>0$ is chosen sufficiently small, then

$$
\left|\left\langle\sigma, \eta^{\prime}-\Pi_{\mathcal{B}} \eta^{\prime}\right\rangle\right| \leq c e^{-\nu-\kappa n} .
$$

By the nondegenerate ends assumption, $\left\|\gamma_{i_{1}}\right\|>0$. Hence (8.25) to (8.29) imply that $\mathfrak{s}_{t}\left(T_{-}, T_{+}, \Sigma\right)(\sigma) \neq 0$ if $n$ is sufficiently large.

Step 4. To complete the proof of Proposition 8.2, we need to handle the case where there is at most one inessential edge incident to $j$. In this case, $j$ has one incoming, inessential edge, and $k \geq 2$ outgoing edges, all of which are essential. Denote the positive leaves corresponding to the essential edges by $i_{1}, \ldots, i_{k}$, where as before, $\nu_{i_{1}}=\nu$.

Pick a leaf $i_{0} \notin\left\{i_{1}, \ldots, i_{k}\right\}$. By Lemma I.2.18, there is a unique, nonvanishing $\sigma \in \operatorname{Coker}\left(D_{\Sigma}\right)$ with

$$
\Pi_{\mathcal{A}} \sigma_{i_{1}}(s, \cdot)=e^{\lambda\left(s-s_{i_{1}}\right)} \gamma_{i_{1}}
$$

and $\Pi_{\mathcal{A}} \sigma_{i}=0$ for $i \notin\left\{i_{0}, i_{1}, i_{2}\right\}$. For each leaf $i$, let $\beta_{i}$ denote the corresponding eigenfunction associated to $\sigma$ via (8.5). In particular, $\beta_{i_{1}}=\gamma_{i_{1}}$.

Proposition I.6.3 tells us (roughly) that if $n$ is large, then near the part of $\Sigma$ that gets collapsed to the vertex $j$ of $\tau_{*}$, the $(0,1)$-form $\sigma$ is well approximated by a nonvanishing cokernel element $\sigma_{Z}$ for a branched cover

$$
\Sigma_{Z} \in \mathcal{M}\left(a_{i_{1}}, \ldots, a_{i_{k}} \mid a_{i_{1}}+\cdots+a_{i_{k}}\right) .
$$

Note that $\sigma_{Z}$ will be an honest cokernel element, and not one of the more general elements of $\widetilde{\operatorname{Coker}}\left(D_{\Sigma_{Z}}\right)$ allowed by Proposition I.6.3, as a consequence of Lemma I.2.20. Combining the precise result of Proposition I.6.3 with Lemma 8.8, we find that for any $\epsilon>0$ if $n$ is sufficiently large, then 
there exists a deck transformation $g$ of the covering $\widetilde{S^{1}} \rightarrow S^{1}$ such that in the notation from Section 8.4,

$$
\zeta_{i_{2}}=-(1+O(\epsilon)) g \cdot \zeta_{i_{1}}
$$

where "O( $\epsilon)$ " here denotes a number with absolute value less than $\epsilon$. Hence the part of $\mathfrak{s}_{0}\left(T_{-}, T_{+}, \Sigma\right)(\sigma)$ coming from the leaves $i_{1}$ and $i_{2}$ is given by

$$
e^{-\nu_{i_{1}}}\left\langle\gamma_{i_{1}}, \beta_{i_{1}}\right\rangle+e^{-\nu_{i_{2}}}\left\langle\gamma_{i_{2}}, \beta_{i_{2}}\right\rangle=e^{-\nu}\left\langle\gamma_{i_{1}}-(1+O(\epsilon)) g \cdot \gamma_{i_{2}}, \gamma_{i_{1}}\right\rangle .
$$

On the other hand, by the nonoverlapping ends assumption, $\gamma_{i_{1}} \neq g \cdot \gamma_{i_{2}}$. Since $\gamma_{i_{1}} \neq 0$, it follows that there is a constant $c>0$ such that if $n$ is sufficiently large, then the expression in (8.30) is greater than $c e^{-\nu}$. A virtual repeat of the arguments in Step 3 concludes that $\mathfrak{s}_{t}\left(T_{-}, T_{+}, \Sigma\right)(\sigma) \neq 0$ if $n$ is sufficiently large. This completes the proof of Proposition 8.2.

\section{Coherent orientations}

This section consists of a lengthy digression on how to "coherently" orient all moduli spaces of unobstructed, immersed, $J$-holomorphic curves in $\mathbb{R} \times Y$, so that the orientations behave well under gluing of the usual kind where there is no obstruction bundle. This is, up to some choices, an established procedure (and one can also allow nonimmersed curves). However, we will need to rework it from a special perspective in order to set up the discussion of signs in obstruction bundle gluing in Section 10.

9.1. Algebraic preliminaries. We begin by reviewing some very basic material about orientations, in order to fix notation.

If $V$ is a finite dimensional vector space over $\mathbb{R}$, let $\mathcal{O}(V)$ denote the set of orientations of $V$. If $\mathfrak{o} \in \mathcal{O}(V)$, we denote the opposite orientation by $-\mathfrak{o}$. If $W$ is another finite dimensional vector space over $\mathbb{R}$, define $\mathcal{O}(V) \otimes \mathcal{O}(W)$ to be the set of pairs $\left(\mathfrak{o}_{V}, \mathfrak{o}_{W}\right) \in \mathcal{O}(V) \times \mathcal{O}(W)$, modulo the relation $\left(\mathfrak{o}_{V}, \mathfrak{o}_{W}\right) \sim$ $\left(-\mathfrak{o}_{V},-\mathfrak{o}_{W}\right)$. There is a canonical isomorphism

$$
\mathcal{O}(V) \otimes \mathcal{O}(W) \stackrel{\simeq}{\longrightarrow} \mathcal{O}(V \oplus W)
$$

obtained by concatenating bases. More generally, an exact sequence of finite dimensional vector spaces over $\mathbb{R}$,

$$
0 \longrightarrow V_{1} \stackrel{f_{1}}{\longrightarrow} V_{2} \stackrel{f_{2}}{\longrightarrow} \cdots \stackrel{f_{k-1}}{\longrightarrow} V_{k} \longrightarrow 0
$$

induces an element

$$
\mathfrak{o}\left(f_{1}, \ldots, f_{k-1}\right) \in \mathcal{O}\left(V_{1}\right) \otimes \cdots \otimes \mathcal{O}\left(V_{k}\right)
$$

defined as follows. Choose a basis $\left(v_{1,1}, \ldots, v_{1, n_{1}}\right)$ of $V_{1}$, and let $\mathfrak{o}_{1} \in \mathcal{O}\left(V_{1}\right)$ denote the corresponding orientation. For $i=2, \ldots, k-1$, choose elements $v_{i, 1}, \ldots, v_{i, n_{i}} \in V_{i}$ such that $\left(f_{i-1}\left(v_{i-1,1}\right), \ldots, f_{i-1}\left(v_{i-1, n_{i-1}}\right), v_{i, 1}, \ldots, v_{i, n_{i}}\right)$ 
is a basis of $V_{i}$, and let $\mathfrak{o}_{i} \in \mathcal{O}\left(V_{i}\right)$ denote the corresponding orientation. Then

$$
\mathfrak{o}\left(f_{1}, \ldots, f_{k-1}\right):=\mathfrak{o}_{1} \otimes \cdots \otimes \mathfrak{o}_{k} .
$$

This orientation does not depend on the choice of the elements $v_{i, j}$; in fact, it is induced by an isomorphism of tensor products of determinant lines

$$
\bigotimes_{i \text { even }} \operatorname{det}\left(V_{i}\right) \simeq \bigotimes_{i \text { odd }} \operatorname{det}\left(V_{i}\right)
$$

which depends only on the long exact sequence (9.2), see e.g., [6]. In addition, (9.3) is invariant under homotopy of exact sequences. That is, if $\left\{f_{i}(t)\right\}_{t \in[0,1]}$ are homotopies of maps $V_{i} \rightarrow V_{i+1}$ for $i=1, \ldots, k-1$, such that the sequence given by $f_{1}(t), \ldots, f_{k-1}(t)$ is exact for each $t \in[0,1]$, then

$$
\mathfrak{o}\left(f_{1}(0), \ldots, f_{k-1}(0)\right)=\mathfrak{o}\left(f_{1}(1), \ldots, f_{k-1}(1)\right) .
$$

If $D$ is a Fredholm operator, define

$$
\mathcal{O}(D):=\mathcal{O}(\operatorname{Ker}(D)) \otimes \mathcal{O}(\operatorname{Coker}(D))
$$

If $\left\{E_{t}\right\}_{t \in[0,1]}$ and $\left\{F_{t}\right\}_{t \in[0,1]}$ are Banach space bundles over $[0,1]$, then a continuous path $\left\{D_{t}: E_{t} \rightarrow F_{t}\right\}_{t \in[0,1]}$ of Fredholm operators induces an isomorphism

$$
\Phi_{\left\{D_{t}\right\}}: \mathcal{O}\left(D_{0}\right) \stackrel{\simeq}{\longrightarrow} \mathcal{O}\left(D_{1}\right),
$$

defined as follows. One can choose decompositions $F_{t} \simeq V_{t} \oplus W_{t}$, depending continuously on $t$, such that $V_{t}$ is finite dimensional, and if $\Pi_{W_{t}}: F_{t} \rightarrow W_{t}$ denotes the projection to $W_{t}$, then $\Pi_{W_{t}} D_{t}$ is surjective. There is then, for each $t$, an exact sequence

$$
0 \longrightarrow \operatorname{Ker}\left(D_{t}\right) \longrightarrow \operatorname{Ker}\left(\Pi_{W_{t}} D_{t}\right) \stackrel{D_{t}}{\longrightarrow} V_{t} \longrightarrow \operatorname{Coker}\left(D_{t}\right) \longrightarrow 0 .
$$

The exact sequence (9.5) induces an isomorphism

$$
\mathcal{O}\left(D_{t}\right) \simeq \mathcal{O}\left(\Pi_{W_{t}} D_{t}\right) \otimes \mathcal{O}\left(V_{t}\right)
$$

Now the family of subspaces $\left\{\operatorname{Ker}\left(\Pi_{W_{t}} D_{t}\right)\right\}_{t \in[0,1]}$ defines a vector bundle over $[0,1]$, as does the family of subspaces $\left\{V_{t}\right\}_{t \in[0,1]}$. These vector bundles induce isomorphisms $\mathcal{O}\left(\Pi_{W_{0}} D_{0}\right) \simeq \mathcal{O}\left(\Pi_{W_{1}} D_{1}\right)$ and $\mathcal{O}\left(V_{0}\right) \simeq \mathcal{O}\left(V_{1}\right)$. Combining these isomorphisms with (9.6) gives the isomorphism (9.4). The latter does not depend on $V_{t}$ and $W_{t}$ and is invariant under homotopy of the path $\left\{D_{t}\right\}$ rel endpoints.

9.2. A linear gluing exact sequence. We now present a variant of the "linear gluing" construction of $[\mathbf{2}, \mathbf{6}]$, designed to fit well in the obstruction bundle context.

We first introduce a class of Fredholm operators that one needs to orient, in order to orient moduli spaces of pseudoholomorphic curves. Fix a positive integer $n$. (The main concern of this paper is the case $n=1$.) 
Definition 9.1. An orientation triple is a triple $\widetilde{C}=\left(C, E,\left\{S_{k}\right\}\right)$, where:

- $C$ is a Riemann surface with cylindrical ends, such that each end is designated "positive" or "negative"; each positive end is identified with $[0, \infty) \times S^{1}$; each negative end is identified with $(-\infty, 0] \times S^{1}$; the positive ends are labeled $1, \ldots, N_{+}$; and the negative ends are labeled $-1, \ldots,-N_{-}$. On each end, denote the $[0, \infty)$ or $(-\infty, 0]$ coordinate by $s$ and the $S^{1}$ coordinate by $t$.

- $E$ is a rank $n$ Hermitian vector bundle over $C$, with a fixed trivialization on each end.

- Associated to the $k$ th end of $C$ is a smooth family $S_{k}(t)$ of symmetric $2 n \times 2 n$ matrices parameterized by $t \in S^{1}$, such that zero is not an eigenvalue of the operator $i \partial_{t}+S_{k}$.

Definition 9.2. For $\widetilde{C}$ as above, define $\mathcal{D}(\widetilde{C})$ to be the set of differential operators $D: C^{\infty}(E) \rightarrow C^{\infty}\left(T^{0,1} C \otimes E\right)$ with the following properties:

- There is a complex structure $j$ on $C$, agreeing with the standard one on the ends, such that in local coordinates and trivializations, $D$ equals $\bar{\partial}$ plus a zeroth order term.

- On the $k$ th end of $C$, write

$$
D \psi=\frac{1}{2}\left(\partial_{s}+i \partial_{t}+M_{k}(s, t)\right) \psi \otimes(d s-i d t),
$$

where $M_{k}(s, t)$ is a $2 n \times 2 n$ matrix. Then $\lim _{|s| \rightarrow \infty} M_{k}(s, \cdot)=S_{k}(\cdot)$ in the sense of $[\mathbf{6}$, Section 2].

It is a standard fact that any such $D$ extends to a Fredholm operator $L_{1}^{2}(E) \rightarrow L^{2}\left(T^{0,1} C \otimes E\right)$. Moreover, a homotopy of such differential operators defines a continuous path of Fredholm operators [6, Prop. 7]. Finally, the space $\mathcal{D}$ is contractible, so for any two operators $D, D^{\prime} \in \mathcal{D}(\widetilde{C})$, there is a canonical bijection $\mathcal{O}(D)=\mathcal{O}\left(D^{\prime}\right)$. We denote this set of orientations by $\mathcal{O}(\widetilde{C})$.

We now consider gluing two orientation triples $\widetilde{C}_{-}=\left(C_{-}, E_{-},\left\{S_{k}^{-}\right\}\right)$ and $\widetilde{C}_{+}=\left(C_{+}, E_{+},\left\{S_{k}^{+}\right\}\right)$. Assume that the first $l$ positive ends of $\widetilde{C}_{-}$ agree with the first $l$ negative ends of $\widetilde{C}_{+}$, in the sense that $S_{k}^{-}=S_{-k}^{+}$for $k=1, \ldots, l$. Fix a large $R>0$. Define a new surface $C$ by identifying, for each $k=1, \ldots, l$, the $s=2 R$ circle in the $k$ th end of $C_{-}$with the $s=-2 R$ circle in the $-k$ th end of $C_{+}$. For each $k=1, \ldots, l$, the part of $C$ coming from the $k$ th end of $C_{-}$and the $-k$ th end of $C_{+}$is a cylinder $Z_{k}$. We identify $Z_{k} \simeq[-2 R, 2 R] \times S^{1}$, so that translation of $s$ by $\pm 2 R$ identifies $Z_{k}$ with the $0 \leq s \leq 4 R$ portion of the $k$ th end of $C_{-}$or with the $-4 R \leq s \leq 0$ of the $-k$ th end of $C_{+}$. Use the fixed trivializations of $E_{-}$and $E_{+}$over the ends to glue them to a bundle $E_{C}$ over $C$. Denote the glued orientation triple by

$$
\widetilde{C}_{-} \#_{l} \widetilde{C}_{+}:=\left(C, E_{C},\left\{S_{k}^{-}\right\}_{k \notin\{1, \ldots, l\}} \cup\left\{S_{k}^{+}\right\}_{k \notin\{-1, \ldots,-l\}}\right) .
$$


Order the positive ends of $C$ so that the positive ends of $C_{+}$come first (in their given order), followed by the unglued positive ends of $C_{-}$(in their given order). Likewise, order the negative ends of $C$ so that the negative ends of $C_{-}$come first (in the order $-1,-2, \ldots$ ), followed by the unglued negative ends of $C_{+}$.

We will use "linear gluing" to define a canonical isomorphism

$$
\mathcal{O}\left(\widetilde{C}_{-}\right) \otimes \mathcal{O}\left(\widetilde{C}_{+}\right)=\mathcal{O}\left(\widetilde{C}_{-} \#_{l} \widetilde{C}_{+}\right) .
$$

Choose operators $D_{-} \in \mathcal{D}\left(\widetilde{C}_{-}\right)$and $D_{+} \in \mathcal{D}\left(\widetilde{C}_{+}\right)$. Let $D_{C} \in \mathcal{D}\left(\widetilde{C}_{-} \#_{l} \widetilde{C}_{+}\right)$ be an operator that agrees with $D_{-}$on $C_{-}$off of the $s \geq R$ part of the first $l$ positive ends and that agrees with $D_{+}$on $C_{+}$off of the $s \leq-R$ part of the first $l$ negative ends. Note that $D_{C}-D_{-}$and $D_{C}-D_{+}$are zeroth order operators on the cylinders $Z_{k}$. Choose a finite dimensional subspace $V_{ \pm}$of $L^{2}\left(T^{0,1} C_{ \pm} \otimes E_{ \pm}\right)$such that if $W_{ \pm}$denotes the orthogonal complement of $V_{ \pm}$, and if $\Pi_{W_{ \pm}}: L^{2}\left(T^{0,1} C_{ \pm} \otimes E_{ \pm}\right) \rightarrow W_{ \pm}$denotes the orthogonal projection, then $\Pi_{W_{ \pm}} \circ D_{ \pm}$is surjective. Here is the version of linear gluing that we will need.

Proposition 9.3. If $R$ is sufficiently large, and if $\left|D_{C}-D_{-}\right|$and $\left|D_{C}-D_{+}\right|$ are sufficiently small on the cylinders $Z_{k}$ for $k=1, \ldots, l$, then there is an exact sequence

$$
\begin{gathered}
0 \longrightarrow \operatorname{Ker}\left(D_{C}\right) \stackrel{f}{\longrightarrow} \operatorname{Ker}\left(\Pi_{W_{-}} D_{-}\right) \oplus \operatorname{Ker}\left(\Pi_{W_{+}} D_{+}\right) \stackrel{g}{\longrightarrow} V_{-} \oplus V_{+} \\
\stackrel{h}{\longrightarrow} \operatorname{Coker}\left(D_{C}\right) \longrightarrow 0
\end{gathered}
$$

Proof. The construction has three steps.

Step 1. We first introduce some notation. Fix a smooth function $\beta: \mathbb{R} \rightarrow$ $[0,1]$ such that $\beta(s)=1$ for $s \leq 0$ and $\beta(s)=0$ for $s \geq 1$. (This notation differs from that of Section 5.2.) Define functions $\beta_{-}, \beta_{+}: C \rightarrow[0,1]$ as follows. On the cylinder $Z_{k}$,

$$
\beta_{-}(s, t):=\beta(s / R), \quad \beta_{+}(s, t):=\beta(-s / R) .
$$

On $C_{-}$off of the first $l$ positive ends, define $\beta_{-}:=1$ and $\beta_{+}:=0$. On $C_{+}$ off of the first $l$ negative ends, define $\beta_{+}:=1$ and $\beta_{-}:=0$.

Now consider $\psi_{-} \in L_{1}^{2}\left(E_{-}\right)$and $\psi_{+} \in L_{1}^{2}\left(E_{+}\right)$, and define

$$
\psi:=\beta_{-} \psi_{-}+\beta_{+} \psi_{+} \in L_{1}^{2}\left(E_{C}\right) .
$$

We can then express

$$
D_{C} \psi=\beta_{-} \Theta_{-}\left(\psi_{-}, \psi_{+}\right)+\beta_{+} \Theta_{+}\left(\psi_{-}, \psi_{+}\right),
$$

where

$$
\begin{aligned}
& \Theta_{-}\left(\psi_{-}, \psi_{+}\right):=D_{-} \psi_{-}+\left(D_{C}-D_{-}\right) \psi_{-}+\left(\bar{\partial} \beta_{+}\right) \psi_{+} \in L^{2}\left(T^{0,1} C_{-} \otimes E_{-}\right), \\
& \Theta_{+}\left(\psi_{-}, \psi_{+}\right):=D_{+} \psi_{+}+\left(D_{C}-D_{+}\right) \psi_{+}+\left(\bar{\partial} \beta_{-}\right) \psi_{-} \in L^{2}\left(T^{0,1} C_{+} \otimes E_{+}\right) .
\end{aligned}
$$


Here we interpret $D_{C}-D_{-}:=0$ off of the support of $\beta_{-}$and $D_{C}-D_{+}:=0$ off of the support of $\beta_{+}$. Note that (9.9) follows from (9.10) because $\beta_{-}=1$ on the support of $d \beta_{+}$and $\beta_{+}=1$ on the support of $d \beta_{-}$.

Step 2. We now prove a key lemma.

Lemma 9.4. Suppose $\psi \in L_{1}^{2}\left(E_{C}\right)$ satisfies $D_{C} \psi=\beta_{-} \theta_{-}+\beta_{+} \theta_{+}$where $\theta_{ \pm} \in L^{2}\left(T^{0,1} C_{ \pm} \otimes E_{ \pm}\right)$. Then there exist unique $\psi_{ \pm} \in L_{1}^{2}\left(E_{ \pm}\right)$such that $\psi=\beta_{-} \psi_{-}+\beta_{+} \psi_{+}$and

$$
\Theta_{-}\left(\psi_{-}, \psi_{+}\right)=\theta_{-}, \quad \Theta_{+}\left(\psi_{-}, \psi_{+}\right)=\theta_{+} .
$$

Proof. We first introduce a linear version of Lemma 7.7. Fix $k \in\{1, \ldots, l\}$, let $S_{k}(t):=S_{k}^{-}(t)=S_{-k}^{+}(t)$, and consider the asymptotic operator

$$
L_{k}:=i \partial_{t}+S_{k}(t): C^{\infty}\left(S^{1} ; \mathbb{R}^{2 n}\right) \rightarrow C^{\infty}\left(S^{1} ; \mathbb{R}^{2 n}\right) .
$$

Let $\Pi_{k+}$ and $\Pi_{k-}$ denote the projections from $L^{2}\left(S^{1} ; \mathbb{R}^{2 n}\right)$ to the sums of the positive and negative eigenspaces of $L_{k}$, respectively. Consider the following operator (where all functions in the various function spaces take values in $\left.\mathbb{R}^{2 n}\right)$ :

$$
\begin{gathered}
\mathcal{F}_{k}: L_{1}^{2}\left([-R, \infty) \times S^{1}\right) \oplus L_{1}^{2}\left((-\infty, R] \times S^{1}\right) \longrightarrow \\
\Pi_{k+} L_{1 / 2}^{2}\left(S^{1}\right) \oplus \Pi_{k-} L_{1 / 2}^{2}\left(S^{1}\right) \oplus L^{2}\left([-R, \infty) \times S^{1}\right) \oplus L^{2}\left((-\infty, R] \times S^{1}\right), \\
\left(\psi_{-}, \psi_{+}\right) \longmapsto\left(\Pi_{k+} \psi_{-}(-R, \cdot), \Pi_{k-} \psi_{+}(R, \cdot), \Theta_{-}\left(\psi_{-}, \psi_{+}\right), \Theta_{+}\left(\psi_{-}, \psi_{+}\right)\right) .
\end{gathered}
$$

Here $\Theta_{-}$and $\Theta_{+}$are defined by identifying $[-R, R] \times S^{1}$ with the $R \leq s \leq 3 R$ portion of the $k$ th positive end of $C_{-}$and with the $-3 R \leq s \leq-R$ portion of the $k$ th negative end of $C_{+}$. A linear version of Lemma 7.7 shows that under the hypotheses of Proposition 9.3, the map $\mathcal{F}_{k}$ is an isomorphism.

Proceeding with the proof of Lemma 9.4, choose an arbitrary decomposition $\psi=\beta_{-} \psi_{-}^{\prime}+\beta_{+} \psi_{+}^{\prime}$ with $\psi_{ \pm}^{\prime} \in L_{1}^{2}\left(E_{ \pm}\right)$. Then by (9.9), we have

$$
\beta_{-}\left(\theta_{-}-\Theta_{-}\left(\psi_{-}^{\prime}, \psi_{+}^{\prime}\right)\right)+\beta_{+}\left(\theta_{+}-\Theta_{+}\left(\psi_{-}^{\prime}, \psi_{+}^{\prime}\right)\right)=0 .
$$

Now write $\psi_{-}=\psi_{-}^{\prime}+\psi_{-}^{\prime \prime}$ and $\psi_{+}=\psi_{+}^{\prime}+\psi_{+}^{\prime \prime}$. Since $\Theta_{ \pm}$is a linear function on $L_{1}^{2}\left(E_{-}\right) \oplus L_{1}^{2}\left(E_{+}\right)$, the desired equation (9.11) is equivalent to the equations

$$
\begin{aligned}
& \Theta_{-}\left(\psi_{-}^{\prime \prime}, \psi_{+}^{\prime \prime}\right)=\theta_{-}-\Theta_{-}\left(\psi_{-}^{\prime}, \psi_{+}^{\prime}\right), \\
& \Theta_{+}\left(\psi_{-}^{\prime \prime}, \psi_{+}^{\prime \prime}\right)=\theta_{+}-\Theta_{+}\left(\psi_{-}^{\prime}, \psi_{+}^{\prime}\right) .
\end{aligned}
$$

By (9.12), the right hand side of (9.13) is supported on the $s \geq R$ portion of the first $l$ positive ends of $C_{-}$, while the right hand side of (9.14) is supported on the $s \leq-R$ portion of the first $l$ negative ends of $C_{+}$. The required $\psi_{-}^{\prime \prime}$ and $\psi_{+}^{\prime \prime}$ are now given on the $s \geq R$ part of the $k$ th positive end of $C_{-}$and on the $s \leq-\mathbb{R}$ part of the $k$ th negative end of $C_{+}$for $k=1, \ldots, l$ by

$$
\left(\psi_{-}^{\prime \prime}, \psi_{+}^{\prime \prime}\right)=\mathcal{F}_{k}^{-1}\left(0,0, \theta_{-}-\Theta_{-}\left(\psi_{-}^{\prime}, \psi_{+}^{\prime}\right), \theta_{+}-\Theta_{+}\left(\psi_{-}^{\prime}, \psi_{+}^{\prime}\right)\right) .
$$

We can, and must, take $\psi_{ \pm}^{\prime \prime}=0$ on the rest of $C_{ \pm}$. 
Step 3. We now define the maps in the sequence (9.7) and prove exactness.

Definition of $f$. Let $\psi \in \operatorname{Ker}\left(D_{C}\right)$ be given. By Lemma 9.4, there exist unique $\psi_{-} \in L_{1}^{2}\left(E_{-}\right)$and $\psi_{+} \in L_{1}^{2}\left(E_{+}\right)$such that $\psi=\beta_{-} \psi_{-}+\beta_{+} \psi_{+}$and

$$
\Theta_{-}\left(\psi_{-}, \psi_{+}\right)=0, \quad \Theta_{+}\left(\psi_{-}, \psi_{+}\right)=0 .
$$

Let $\phi_{ \pm}$denote the $L^{2}$-orthogonal projection of $\psi_{ \pm}$onto $\operatorname{Ker}\left(\Pi_{W_{ \pm}} D_{ \pm}\right)$. Define

$$
f(\psi):=\left(\phi_{-}, \phi_{+}\right) .
$$

Definition of $g$. Let $\phi_{-} \in \operatorname{Ker}\left(\Pi_{W_{-}} D_{-}\right)$and $\phi_{+} \in \operatorname{Ker}\left(\Pi_{W_{+}} D_{+}\right)$be given. We claim that there are unique $\zeta_{-} \in L_{1}^{2}\left(E_{-}\right)$and $\zeta_{+} \in L_{1}^{2}\left(E_{+}\right)$such that $\zeta_{-}$is $L^{2}$-orthogonal to $\operatorname{Ker}\left(\Pi_{W_{-}} D_{-}\right), \zeta_{+}$is $L^{2}$-orthogonal to $\operatorname{Ker}\left(\Pi_{W_{+}} D_{+}\right)$ and the pair

$$
\left(\psi_{-}, \psi_{+}\right):=\left(\phi_{-}+\zeta_{-}, \phi_{+}+\zeta_{+}\right)
$$

solves the equations

$$
\Pi_{W_{-}} \Theta_{-}\left(\psi_{-}, \psi_{+}\right)=0, \quad \Pi_{W_{+}} \Theta_{+}\left(\psi_{-}, \psi_{+}\right)=0 .
$$

To see this, let $F_{-}: W_{-} \rightarrow \operatorname{Ker}\left(\Pi_{W_{-}} D_{-}\right)^{\perp}$ denote the inverse of $\Pi_{W_{-}} D_{-}$, and let $F_{+}: W_{+} \rightarrow \operatorname{Ker}\left(\Pi_{W_{+}} D_{+}\right)^{\perp}$ denote the inverse of $\Pi_{W_{+}} D_{+}$. Then applying $F_{-}$and $F_{+}$to the first and second equations in (9.17), respectively, we obtain a pair of equations which can be written as

$$
\begin{gathered}
\left(\begin{array}{cc}
1+F_{-} \Pi_{W_{-}}\left(D_{C}-D_{-}\right) & F_{-} \Pi_{W_{-}}\left(\bar{\partial} \beta_{+}\right) \\
F_{+} \Pi_{W_{+}}\left(\bar{\partial} \beta_{-}\right) & 1+F_{+} \Pi_{W_{+}}\left(D_{C}-D_{+}\right)
\end{array}\right)\left(\begin{array}{c}
\zeta_{-} \\
\zeta_{+}
\end{array}\right) \\
=\left(\begin{array}{l}
-F_{-} \Pi_{W_{-}}\left(\left(D_{C}-D_{-}\right) \phi_{-}+\left(\bar{\partial} \beta_{+}\right) \phi_{+}\right) \\
-F_{+} \Pi_{W_{+}}\left(\left(D_{C}-D_{+}\right) \phi_{+}+\left(\bar{\partial} \beta_{-}\right) \phi_{-}\right)
\end{array}\right) .
\end{gathered}
$$

If $\left|D_{C}-D_{-}\right|$and $\left|D_{C}-D_{+}\right|$are sufficiently small, and $R$ is sufficiently large, with respect to the operator norms of $F_{+}$and $F_{-}$, then these equations have a unique solution. In terms of this unique solution, define

$$
g\left(\phi_{-}, \phi_{+}\right):=\left(\Theta_{-}\left(\psi_{-}, \psi_{+}\right), \Theta_{+}\left(\psi_{-}, \psi_{+}\right)\right) \text {. }
$$

Definition of $h$. Given $\left(\theta_{-}, \theta_{+}\right) \in V_{-} \oplus V_{+}$, define $h\left(\theta_{-}, \theta_{+}\right)$to be the equivalence class of $\beta_{-} \theta_{-}+\beta_{+} \theta_{+}$in $\operatorname{Coker}\left(D_{C}\right)$.

$f$ is injective. Let $\psi \in \operatorname{Ker}\left(D_{C}\right)$ and suppose that $f(\psi)=0$. This means that $\psi=\beta_{-} \zeta_{-}+\beta_{+} \zeta_{-}$, where $\zeta_{ \pm}$is $L^{2}$-orthogonal to $\operatorname{Ker}\left(\Pi_{W_{ \pm}} D_{ \pm}\right)$ and $\Theta_{ \pm}\left(\zeta_{-}, \zeta_{+}\right)=0$. By uniqueness of the solution to equation (9.17) for $\phi_{-}=\phi_{+}=0$, it follows that $\zeta_{-}=\zeta_{+}=0$, and so $\psi=0$.

$\operatorname{Im}(f) \subset \operatorname{Ker}(g)$ : Immediate from the definitions.

$\operatorname{Ker}(g) \subset \operatorname{Im}(f)$ : Suppose $g\left(\phi_{-}, \phi_{+}\right)=0$. This means that there exists $\zeta_{ \pm}$orthogonal to $\operatorname{Ker}\left(\Pi_{W_{ \pm}} D_{ \pm}\right)$such that the pair $\left(\psi_{-}, \psi_{+}\right)$defined in (9.16) satisfies equation (9.15). Then $\psi:=\beta_{-} \psi_{-}+\beta_{+} \psi_{+}$is in $\operatorname{Ker}\left(D_{C}\right)$ by equation (9.9), and by definition $f(\psi)=\left(\phi_{-}, \phi_{+}\right)$.

$\operatorname{Im}(g) \subset \operatorname{Ker}(h):$ Immediate from (9.9). 
$\operatorname{Ker}(h) \subset \operatorname{Im}(g)$ : Let $\left(\theta_{-}, \theta_{+}\right) \in V_{-} \oplus V_{+}$be given, and suppose that $h\left(\theta_{-}, \theta_{+}\right)=0$. This means that there exists $\psi \in L_{1}^{2}\left(E_{C}\right)$ with

$$
D_{C} \psi=\beta_{-} \theta_{-}+\beta_{+} \theta_{+} .
$$

By Lemma 9.4, there exist $\psi_{ \pm} \in L_{1}^{2}\left(E_{ \pm}\right)$such that

$$
\left(\Theta_{-}\left(\psi_{-}, \psi_{+}\right), \Theta_{+}\left(\psi_{-}, \psi_{+}\right)\right)=\left(\theta_{-}, \theta_{+}\right) .
$$

Let $\phi_{ \pm}$denote the $L^{2}$-orthogonal projection of $\psi_{ \pm}$onto $\operatorname{Ker}\left(\Pi_{W_{ \pm}} D_{ \pm}\right)$. Then by definition, $g\left(\phi_{-}, \phi_{+}\right)=\left(\theta_{-}, \theta_{+}\right)$.

$h$ is surjective. Given $\xi \in L^{2}\left(E_{C}\right)$, we need to find $\left(\theta_{-}, \theta_{+}\right) \in V_{-} \oplus V_{+}$ and $\psi \in L_{1}^{2}(E)$ such that

$$
D_{C} \psi+\beta_{-} \theta_{-}+\beta_{+} \theta_{+}=\xi .
$$

Choose any decomposition $\xi=\beta_{-} \xi_{-}+\beta_{+} \xi_{+}$with $\xi_{ \pm} \in L^{2}\left(T^{0,1} C_{ \pm} \otimes E_{ \pm}\right)$. Since $\Pi_{W_{ \pm}} D_{ \pm}$is surjective, there exist $\psi_{ \pm} \in L_{1}^{2}\left(E_{ \pm}\right)$and $\theta_{ \pm} \in V_{ \pm}$such that

$$
D_{-} \psi_{-}+\theta_{-}=\xi_{-}, \quad D_{+} \psi_{+}+\theta_{+}=\xi_{+} .
$$

Now write $\psi=\beta_{-}\left(\psi_{-}+\psi_{-}^{\prime}\right)+\beta_{+}\left(\psi_{+}+\psi_{+}^{\prime}\right)$. Then to solve the desired equation (9.18), it is enough to find $\psi_{ \pm}^{\prime}$ such that

$$
\begin{aligned}
& \Theta_{-}\left(\psi_{-}^{\prime}, \psi_{+}^{\prime}\right):=-\Theta_{-}\left(\psi_{-}, \psi_{+}\right)-\theta_{-}+\xi_{-}, \\
& \Theta_{+}\left(\psi_{-}^{\prime}, \psi_{+}^{\prime}\right):=-\Theta_{+}\left(\psi_{-}, \psi_{+}\right)-\theta_{+}+\xi_{+} .
\end{aligned}
$$

By (9.10) and (9.19), the right hand side of (9.20) is supported in the $s \geq R$ portion of the first $l$ positive ends of $C_{-}$, while the right hand side of (9.21) is supported in the $s \leq-R$ portion of the first $l$ negative ends of $C_{+}$. It follows that as in the proof of Lemma 9.4, we can use the maps $\mathcal{F}_{k}^{-1}$ to find the required $\psi_{-}^{\prime}$ and $\psi_{+}^{\prime}$.

Remark 9.5. Counting dimensions in the exact sequence (9.3) recovers the standard fact that ind $\left(D_{C}\right)=\operatorname{ind}\left(D_{-}\right)+\operatorname{ind}\left(D_{+}\right)$.

9.3. Gluing orientations. Proposition 9.3 allows us to glue orientations as follows. The exact sequence (9.3) induces an isomorphism

$$
\mathcal{O}\left(D_{C}\right) \simeq \mathcal{O}\left(\Pi_{W_{-}} D_{-}\right) \otimes \mathcal{O}\left(\Pi_{W_{+}} D_{+}\right) \otimes \mathcal{O}\left(V_{-}\right) \otimes \mathcal{O}\left(V_{+}\right) .
$$

Combining this with the $D_{-}$and $D_{+}$versions of (9.6), we obtain an isomorphism

$$
\mathcal{O}\left(D_{-}\right) \otimes \mathcal{O}\left(D_{+}\right) \simeq \mathcal{O}\left(D_{C}\right)
$$

Lemma 9.6. The isomorphism (9.23) does not depend on $V_{-}$and $V_{+}$, and is invariant under homotopy of the data $\left(D_{-}, D_{+} ; R, D_{C}\right)$, so that it induces a well-defined isomorphism

$$
\mathcal{O}\left(\widetilde{C}_{-}\right) \otimes \mathcal{O}\left(\widetilde{C}_{+}\right) \simeq \mathcal{O}\left(\widetilde{C}_{-} \#_{l} \widetilde{C}_{+}\right) .
$$


Proof. The proof has three steps.

Step 1. To prove that (9.23) is homotopy invariant, the main difficulty is that the dimensions of $\operatorname{Ker}\left(D_{C}\right)$ and $\operatorname{Coker}\left(D_{C}\right)$ may jump during a homotopy. To deal with this issue, we first give an alternate description of the isomorphism (9.23), which does not directly refer to the kernel or cokernel of $D_{C}$.

Continuing with the notation from the proof of Proposition 9.3, define $V_{C} \subset L^{2}\left(T^{0,1} C \otimes E_{C}\right)$ to be the subspace consisting of sections $\beta_{-} \theta_{-}+\beta_{+} \theta_{+}$ where $\theta_{ \pm} \in V_{ \pm}$. Assume that no nonzero element of $V_{-}$or $V_{+}$is supported entirely on the $|s| \geq R$ part of the first $l$ positive or negative ends, so that the map $V_{-} \oplus V_{+} \rightarrow V_{C}$ sending $\left(\theta_{-}, \theta_{+}\right) \mapsto \beta_{-} \theta_{-}+\beta_{+} \theta_{+}$is an isomorphism. (In general, one can arrange this by a slight perturbation of $V_{ \pm}$.) Let $W_{C}$ denote the orthogonal complement of $V_{C}$, and let $\Pi_{W_{C}}: L^{2}\left(T^{0,1} C \otimes E_{C}\right) \rightarrow W_{C}$ denote the orthogonal projection. Note that $\Pi_{W_{C}} D_{C}$ is surjective, because the map $h$ in (9.3) is surjective. So as in (9.5), there is an exact sequence

$$
0 \longrightarrow \operatorname{Ker}\left(D_{C}\right) \longrightarrow \operatorname{Ker}\left(\Pi_{W_{C}} D_{C}\right) \stackrel{D_{C}}{\longrightarrow} V_{C} \longrightarrow \operatorname{Coker}\left(D_{C}\right) \longrightarrow 0 .
$$

We now define a map

$$
\Phi: \operatorname{Ker}\left(\Pi_{W_{C}} D_{C}\right) \longrightarrow \operatorname{Ker}\left(\Pi_{W_{-}} D_{-}\right) \oplus \operatorname{Ker}\left(\Pi_{W_{+}} D_{+}\right)
$$

as follows. Suppose $\psi \in \operatorname{Ker}\left(\Pi_{W_{C}} D_{C}\right)$. This means that there exist unique $\theta_{ \pm} \in V_{ \pm}$such that

$$
D_{C} \psi=\beta_{-} \theta_{-}+\beta_{+} \theta_{+} .
$$

By Lemma 9.4, there are unique $\psi_{ \pm} \in L_{1}^{2}\left(E_{ \pm}\right)$satisfying equations (9.8) and (9.11). Now let $\phi_{ \pm}$denote the $L^{2}$-orthogonal projection of $\psi_{ \pm}$onto $\operatorname{Ker}\left(\Pi_{W_{ \pm}} D_{ \pm}\right)$, and define

$$
\Phi(\psi):=\left(\phi_{-}, \phi_{+}\right) .
$$

It follows directly from the definitions that the exact sequences (9.7) and (9.24) fit into a commutative diagram

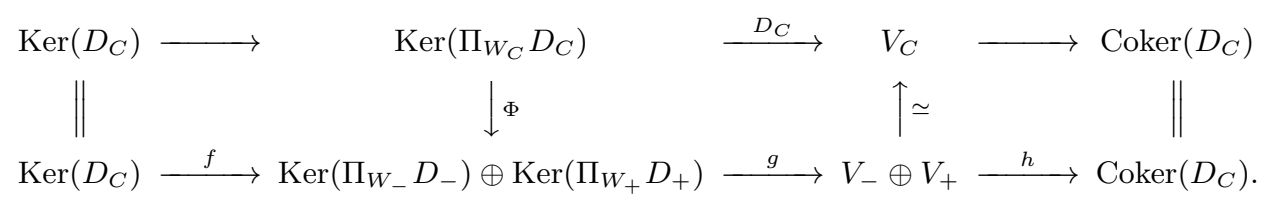

By the five lemma, $\Phi$ is an isomorphism, and hence induces an isomorphism

$$
\mathcal{O}\left(\Pi_{W_{C}} D_{C}\right) \simeq \mathcal{O}\left(\Pi_{W_{-}} D_{-}\right) \otimes \mathcal{O}\left(\Pi_{W_{+}} D_{+}\right) .
$$

Moreover, it follows from the above commutative diagram that under the canonical isomorphisms (9.6) for $D_{ \pm}$and $D_{C}$, the isomorphism (9.26) agrees with (9.23).

Step 2. We now show that the isomorphism (9.23) does not depend on $V_{-}$and $V_{+}$. For this purpose, it is enough to show that the isomorphism 
(9.23) is unchanged under replacing $V_{-}$and $V_{+}$by larger subspaces $V_{-}^{\prime} \supset V_{-}$ and $V_{+}^{\prime} \supset V_{+}$. It follows directly from the definitions that the primed and unprimed versions of $\Phi$ fit into a commutative diagram

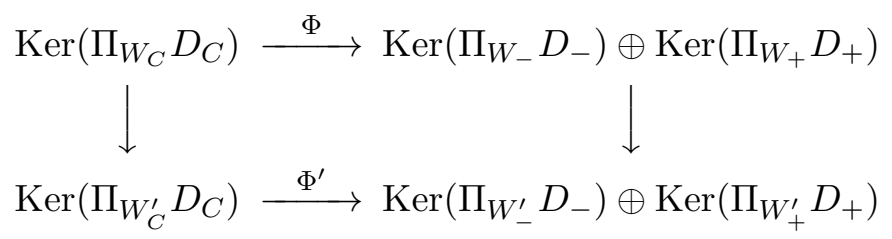

where the vertical maps are the inclusions. It follows readily that the primed and unprimed versions of the isomorphism (9.23) agree.

Step 3. We now prove homotopy invariance. Given a homotopy

$$
\left\{\left(D_{-}(t), D_{+}(t), D_{C}(t)\right)\right\}_{t \in[0,1]}
$$

of triples of operators, we need to prove that the diagram

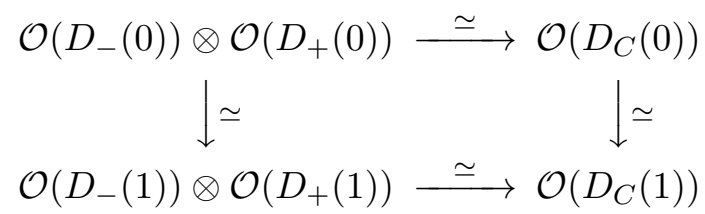

commutes, where the horizontal isomorphisms are the $t=0$ and $t=1$ versions of (9.23), while the vertical isomorphisms are defined in (9.4). To do so, choose subspaces $V_{ \pm}(t)$ with the required properties that depend continuously on $t$. Then the family of maps

$$
\left\{\Phi_{t}: \operatorname{Ker}\left(\Pi_{W_{C}(t)} D_{C}(t)\right) \longrightarrow \operatorname{Ker}\left(\Pi_{W_{-}(t)} D_{-}(t)\right) \oplus \operatorname{Ker}\left(\Pi_{W_{+}(t)} D_{+}(t)\right)\right\}_{t \in[0,1]}
$$

defines an isomorphism of vector bundles over $[0,1]$. It now follows after unraveling the definitions that the diagram (9.27) commutes.

If $\mathfrak{o}_{-} \in \mathcal{O}\left(\widetilde{C}_{-}\right)$and $\mathfrak{o}_{+} \in \mathcal{O}\left(\widetilde{C}_{+}\right)$, denote the corresponding glued orientation by

$$
\mathfrak{o}_{-} \#_{l} \mathfrak{o}_{+} \in \mathcal{O}\left(\widetilde{C}_{-} \#_{l} \widetilde{C}_{+}\right) .
$$

9.4. Associativity of linear gluing. We now show that the operation of gluing orientations is associative. More precisely, consider three orientation triples $\widetilde{C_{-}}, \widetilde{C}_{0}, \widetilde{C_{+}}$, such that the first $l_{-}$negative ends of $\widetilde{C}_{0}$ (numbered $\left.-1, \ldots, l_{-}\right)$agree with the first $l_{-}$positive ends of $\widetilde{C}_{-}$in that order, while the first $l_{+}$positive ends of $\widetilde{C}_{0}$ likewise agree with the first $l_{+}$negative ends of $\widetilde{C}_{+}$.

Lemma 9.7. If $\mathfrak{o}_{ \pm} \in \mathcal{O}\left(\widetilde{C}_{ \pm}\right)$and $\mathfrak{o}_{0} \in \mathcal{O}\left(\widetilde{C}_{0}\right)$, then

$$
\left(\mathfrak{o}_{-} \#_{l_{-}} \mathfrak{o}_{0}\right) \#_{l_{+}} \mathfrak{o}_{+}=\mathfrak{o}_{-} \#_{l_{-}}\left(\mathfrak{o}_{0} \#_{l_{+}} \mathfrak{o}_{+}\right)
$$

in $\mathcal{O}\left(\widetilde{C}_{-} \#_{l_{-}} \widetilde{C}_{0} \#_{l_{+}} \widetilde{C}_{+}\right)$. 
Proof. The plan is to relate both sides of (9.28) to an analog of linear gluing which glues the three orientation triples $\widetilde{C}_{-}, \widetilde{C}_{0}, \widetilde{C}_{+}$together simultaneously.

Step 1. We begin by explaining the triple linear gluing operation, in somewhat more detail than is necessary here, because it will play an important role in the proof of Theorem 10.2 in Section 10.5.

Let $C:=C_{-} \#_{l_{-}} C_{0} \#_{l_{+}} C_{+}$denote the glued curve, and define functions $\beta_{-}, \beta_{0}, \beta_{+}: C \rightarrow[0,1]$ as follows. For $k \in\left\{-1, \ldots,-l_{-}\right\}$, let $Z_{k}$ denote the cylinder where the $-k$ th end of $C_{-}$is glued to the $k$ th end of $C_{0}$. For $k \in\left\{1, \ldots, l_{+}\right\}$, let $Z_{k}$ denote the cylinder where the $k$ th end of $C_{0}$ is glued to the $-k$ th end of $C_{+}$. For each $k$, identify

$$
Z_{k} \simeq[-2 R, 2 R] \times S^{1} .
$$

On the cylinder $Z_{k}$ for $k \in\left\{-1, \ldots,-l_{-}\right\}$, define

$$
\beta_{-}(s, t):=\beta(s / R), \quad \beta_{0}(s, t):=\beta(-s / R), \quad \beta_{+}(s, t):=0,
$$

where $\beta$ is the function defined in Section 9.2. On the cylinder $Z_{k}$ for $k \in\left\{1, \ldots, l_{+}\right\}$, define

$$
\beta_{-}(s, t):=0, \quad \beta_{0}(s, t):=\beta(s / R), \quad \beta_{+}(s, t):=\beta(-s / R) .
$$

On $C_{-}$, off of the first $l_{-}$positive ends, define $\beta_{-}:=1$ and $\beta_{0}, \beta_{+}:=0$. On $C_{0}$, off the first $l_{-}$negative ends and the first $l_{+}$positive ends, define $\beta_{0}:=1$ and $\beta_{-}, \beta_{+}:=0$. On $C_{+}$, off of the first $l_{+}$negative ends, define $\beta_{+}:=1$ and $\beta_{-}, \beta_{0}:=0$.

Choose operators $D_{ \pm} \in \mathcal{D}\left(\widetilde{C}_{ \pm}\right)$and $D_{0} \in \mathcal{D}\left(\widetilde{C}_{0}\right)$. Let

$$
D_{C} \in \mathcal{D}\left(\widetilde{C}_{-} \#_{l_{-}} \widetilde{C}_{0} \#_{l_{+}} \widetilde{C}_{+}\right)
$$

be an operator that agrees with $D_{-}$on $C_{-}$off of the first $l_{-}$positive ends agrees with $D_{0}$ on $C_{0}$ off of the first $l_{-}$negative ends and the first $l_{+}$positive ends and agrees with $D_{+}$on $C_{+}$off of the first $l_{+}$negative ends. Assume that $R$ is large and that $\left|D_{C}-D_{ \pm}\right|$and $\left|D_{C}-D_{0}\right|$ are small on the cylinders $Z_{k}$ for $k \in\left\{ \pm 1, \ldots, \pm l_{ \pm}\right\}$.

Now consider $\psi_{ \pm} \in L_{1}^{2}\left(E_{ \pm}\right)$and $\psi_{0} \in L_{1}^{2}\left(E_{0}\right)$, and define

$$
\psi:=\beta_{-} \psi_{-}+\beta_{0} \psi_{0}+\beta_{+} \psi_{+} \in L_{1}^{2}\left(E_{C}\right) .
$$

We can then express

$$
D_{C} \psi=\beta_{-} \Theta_{-}\left(\psi_{-}, \psi_{0}\right)+\beta_{0} \Theta_{0}\left(\psi_{-}, \psi_{0}, \psi_{+}\right)+\beta_{+} \Theta_{+}\left(\psi_{0}, \psi_{+}\right),
$$

where the $\Theta$ 's are defined by the obvious analog of (9.10).

Now choose finite dimensional subspaces $V_{ \pm} \subset L^{2}\left(T^{0,1} C_{ \pm} \otimes E_{ \pm}\right)$and $V_{0} \subset L^{2}\left(T^{0,1} C_{0} \otimes E_{0}\right)$ such that if $W_{ \pm}$and $W_{0}$ denote their orthogonal complements, then $\Pi_{W_{ \pm}} D_{ \pm}$and $\Pi_{W_{0}} D_{0}$ are surjective. Then the exact 
sequence (9.7) has a straightforward generalization to an exact sequence $0 \longrightarrow \operatorname{Ker}\left(D_{C}\right) \longrightarrow \operatorname{Ker}\left(\Pi_{W_{-}} D_{-}\right) \oplus \operatorname{Ker}\left(\Pi_{W_{0}} D_{0}\right) \oplus \operatorname{Ker}\left(\Pi_{W_{+}} D_{+}\right) \longrightarrow$

$$
\longrightarrow V_{-} \oplus V_{0} \oplus V_{+} \longrightarrow \operatorname{Coker}\left(D_{C}\right) \longrightarrow 0 \text {. }
$$

As before, this exact sequence induces a homotopy invariant isomorphism

$$
\mathcal{O}\left(D_{-}\right) \otimes \mathcal{O}\left(D_{0}\right) \otimes \mathcal{O}\left(D_{+}\right) \simeq \mathcal{O}\left(D_{C}\right)
$$

As in Section 9.3, one can give an alternate description of the isomorphism (9.31) as follows. Let

$$
V_{C}:=\left\{\beta_{-} \theta_{-}+\beta_{0} \theta_{0}+\beta_{+} \theta_{+} \mid \theta_{-} \in V_{-}, \theta_{0} \in V_{0}, \theta_{+} \in V_{+}\right\} .
$$

Choose $V_{ \pm}, V_{0}$ such that the map $V_{-} \oplus V_{0} \oplus V_{+} \rightarrow V_{C}$ is an isomorphism, and let $W_{C}$ denote the orthogonal complement of $V_{C}$. The map (9.25) then has an obvious analog

$$
\widetilde{\Phi}: \operatorname{Ker}\left(\Pi_{W_{C}} D_{C}\right) \longrightarrow \operatorname{Ker}\left(\Pi_{W_{-}} D_{-}\right) \oplus \operatorname{Ker}\left(\Pi_{W_{0}} D_{0}\right) \oplus \operatorname{Ker}\left(\Pi_{W_{+}} D_{+}\right),
$$

which is an isomorphism and induces the map (9.31) on orientations.

Step 2. We now relate triple linear gluing to the composition of two ordinary linear gluings. Let $D_{0-}$ denote the operator on $C_{-} \#_{l_{-}} C_{0}$ that agrees with $D_{0}$ on the ends $1, \ldots, l$ of $C_{0}$ and that agrees with $D_{C}$ on the rest of $C_{-} \#_{l_{-}} C_{0}$. Let $\beta_{-}, \beta_{0-}: C_{-} \#_{l_{-}} C_{0} \rightarrow[0,1]$ denote the cutoff functions for this gluing, and let

$$
V_{0-}:=\left\{\beta_{-} \theta_{-}+\beta_{0-} \theta_{0} \mid \theta_{-} \in V_{-}, \theta_{0} \in V_{0}\right\} .
$$

Define $D_{0+}, \beta_{0+}, V_{0+}$ likewise for $C_{0} \#_{l_{+}} C_{+}$. Tracing through the definitions shows that the diagram

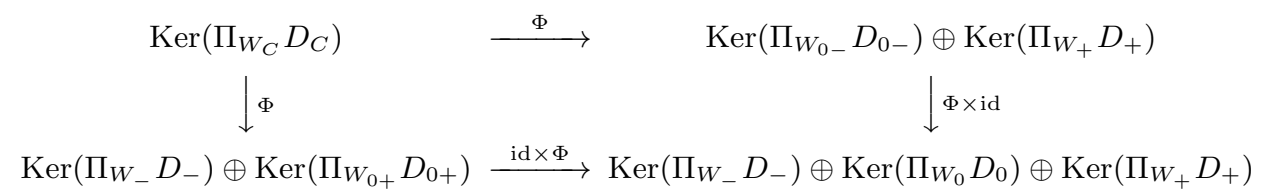

commutes, because both compositions are equal to the map $\widetilde{\Phi}$. The lemma follows.

9.5. Orienting the moduli spaces. We now use the linear gluing operation to orient all moduli spaces of immersed, unobstructed pseudoholomorphic curves in $\mathbb{R} \times Y$, so that the orientations behave well under gluing. We follow the approach of $[\mathbf{2}]$, but with some slightly different choices. 
Orienting the Fredholm operators. For each orientation triple $\widetilde{C}=$ $\left(C, E,\left\{S_{k}\right\}\right)$, we will choose an orientation $\mathfrak{o}_{\widetilde{C}} \in \mathcal{O}(\widetilde{C})$. We want these choices to satisfy four axioms. The first axiom concerns the complex linear case:

(OR1) If $C$ has no ends, and if $D \in \mathcal{D}(\widetilde{C})$ is $\mathbb{C}$-linear, then $\mathfrak{o}_{\widetilde{C}}$ corresponds to the canonical orientation of $\mathcal{O}(D)$ coming from the complex vector space structure on $\operatorname{Ker}(D)$ and $\operatorname{Coker}(D)$.

The second axiom describes the behavior of the orientations under "complete" gluing, where we glue all the positive ends of one curve to all the negative ends of another:

(OR2) If $\widetilde{C}_{-}$has exactly $l$ positive ends, if $\widetilde{C}_{+}$has exactly $l$ negative ends and if the $k$ th positive end of $\widetilde{C}_{-}$agrees with the $k$ th negative end of $\widetilde{C}_{+}$for each $k=1, \ldots, l$, then

$$
\mathfrak{o}_{\left(\widetilde{C}_{-} \#_{l} \widetilde{C}_{+}\right)}=\mathfrak{o}_{\widetilde{C}_{-}} \#{ }_{l} \mathfrak{o}_{\widetilde{C}_{+}} \cdot
$$

Before stating the third axiom, we need to introduce some mod 2 indices. Consider a loop of symmetric $2 n \times 2 n$ matrices $\{S(t)\}_{t \in S^{1}}$ such that zero is not an eigenvalue of the operator $i \partial_{t}+S$. Define

$$
\varepsilon(s):=n+\mu\left(\{\Psi(t)\}_{t \in[0,2 \pi]}\right) \quad \bmod 2,
$$

where $\Psi(t)$ is the path of symplectic matrices with $\Phi(0)=1$ generated by $S(t)$ as in equation (I.2.7), and $\mu$ denotes the Maslov index. Given an orientation triple $\widetilde{C}=\left(C, E,\left\{S_{k}\right\}\right)$, define

$$
\varepsilon_{-}(\widetilde{C}):=\sum_{k=-1}^{-N_{-}} \varepsilon\left(S_{k}\right), \quad \varepsilon_{+}(\widetilde{C}):=\sum_{k=1}^{N_{+}} \varepsilon\left(S_{k}\right), \quad \varepsilon(\widetilde{C}):=\varepsilon_{+}(\widetilde{C})-\varepsilon_{-}(\widetilde{C}) .
$$

The third axiom concerns the disjoint union $\widetilde{C}_{1} \sqcup \widetilde{C}_{2}$ of two orientation triples $\widetilde{C}_{1}$ and $\widetilde{C}_{2}$. Here the positive or negative ends of $\widetilde{C}_{1} \sqcup \widetilde{C}_{2}$ are ordered so that the ends of $\widetilde{C}_{1}$ come first, followed by the ends of $\widetilde{C}_{2}$, in their given order. If $D_{1} \in \mathcal{D}\left(\widetilde{C}_{1}\right)$ and $D_{2} \in \mathcal{D}\left(\widetilde{C}_{2}\right)$, then (9.1) defines a canonical isomorphism $\mathcal{O}\left(D_{1} \oplus D_{2}\right)=\mathcal{O}\left(D_{1}\right) \otimes \mathcal{O}\left(D_{2}\right)$, and hence $\mathcal{O}\left(\widetilde{C}_{1} \sqcup \widetilde{C}_{2}\right)=\mathcal{O}\left(\widetilde{C}_{1}\right) \otimes \mathcal{O}\left(\widetilde{C}_{2}\right)$. The axiom is now:

(OR3)

$$
\mathfrak{o}_{\widetilde{C}_{1} \sqcup \widetilde{C}_{2}}=(-1)^{\varepsilon-\left(\widetilde{C}_{1}\right) \varepsilon\left(\widetilde{C}_{2}\right)} \mathfrak{o}_{\widetilde{C}_{1}} \otimes \mathfrak{o}_{\widetilde{C}_{2}}
$$

To find orientations satisfying the above three axioms, we first choose arbitrary orientations for certain special orientation triples. For each path of symmetric matrices $\{S(t)\}_{t \in S^{1}}$ as above, consider the orientation triple

$$
\widetilde{C}_{S}:=\left(\mathbb{C}, \mathbb{C} \times \mathbb{C}^{n}, S(\cdot)\right),
$$

where the end of $\mathbb{C}$ is identified with $[0, \infty) \times S^{1}$ via the exponential function. 
Lemma 9.8. Given orientation choices $\mathfrak{o}_{\widetilde{C}_{S}}$ for each $S$ as above, there is a unique way to extend these to choose orientations $\mathfrak{o}_{\widetilde{C}}$ for all orientation triples $\widetilde{C}=\left(C, E,\left\{S_{k}\right\}\right)$, such that axioms (OR1) to (OR3) hold.

Proof. This follows from the argument in [2, §3], using the associativity property (9.28). Note that to translate between the conventions in [2] and those here, one needs to reverse the ordering of the negative ends of each $C$.

Orienting the moduli spaces. We now explain how a system of orientations as above with $n=1$ orients the moduli spaces of immersed, unobstructed $J$-holomorphic curves in $\mathbb{R} \times Y$. Fix a parameterization of each Reeb orbit by a map $\alpha: S^{1} \rightarrow \mathbb{R} \times Y$ such that $\partial_{t} \alpha$ is a constant positive multiple of the Reeb vector field. Also fix a trivialization of the contact plane field $\xi$ over each Reeb orbit in $Y$. In this trivialization, the linearized Reeb flow on the contact planes along $\alpha$ is given by $\partial_{t}-J_{0} S_{\alpha}(t)$ where $S_{\alpha}(t)$ is a $2 \times 2$ symmetric matrix and $J_{0}:=\left(\begin{array}{cc}0 & -1 \\ 1 & 0\end{array}\right)$.

Now let $\alpha_{+}=\left(\alpha_{1}, \ldots, \alpha_{N_{+}}\right)$and $\alpha_{-}=\left(\alpha_{-1}, \ldots, \alpha_{-N_{+}}\right)$be ordered lists of Reeb orbits, let $C \in \mathcal{M}^{J}\left(\alpha_{+}, \alpha_{-}\right)$be immersed and let $N_{C}$ denote the normal bundle to $C$. Over the $k$ th end of $C$, trivialize $N_{C}$ by using a choice of coordinates $(z, w)$ in a neighborhood of the corresponding Reeb orbit provided by Lemma 2.1, and identifying $N_{C}$ with the tangent spaces to the $z=$ constant disks. Then the deformation operator $D_{C}$ is an element of the space $\mathcal{D}(\widetilde{C})$ where $\widetilde{C}=\left(C, N_{C},\left\{S_{\alpha_{k}}\right\}\right)$. Thus the chosen orientation $\mathfrak{o}_{\widetilde{C}}$ determines an orientation in $\mathcal{O}\left(D_{C}\right)$. If $C$ is unobstructed so that $\operatorname{Coker}\left(D_{C}\right)=\{0\}$, then this orients $\operatorname{Ker}\left(D_{C}\right)=T_{C} \mathcal{M}^{J}\left(\alpha_{+}, \alpha_{-}\right)$.

Definition 9.9. A system of coherent orientations of the moduli spaces of immersed unobstructed $J$-holomorphic curves in $\mathbb{R} \times Y$ is a system of orientations determined as above from orientations $\mathfrak{o}_{\widetilde{C}}$ for all $\widetilde{C}$ with $n=1$ satisfying axioms (OR1) to (OR3) above, together with axiom (OR4) below.

To state axiom (OR4), consider a family $\{S(t)\}_{t \in S^{1}}$ of $2 \times 2$ symmetric matrices such that the associated symplectic matrix $\Phi(2 \pi)$ is elliptic, i.e., has eigenvalues $e^{ \pm 2 \pi i \theta}$ for some $\theta \in \mathbb{R} \backslash \mathbb{Z}$. We now describe a canonical orientation in $\mathcal{O}\left(\widetilde{C}_{S}\right)$.

First consider the case where $S(t)=\theta$ for all $t$. Then there is a canonical orientation in $\mathcal{O}\left(\widetilde{C}_{\theta}\right)$, because the operator

$$
D:=\bar{\partial}+\theta d \bar{z} \in \mathcal{D}\left(\widetilde{C}_{\theta}\right)
$$


is complex linear. In general, if $\Phi(2 \pi)$ is elliptic, then there is some $\theta \in \mathbb{R} \backslash \mathbb{Z}$ such that

$\left(^{*}\right)$ the path $\{\Phi(t)\}_{t \in[0,2 \pi]}$ is homotopic rel endpoints to $\left\{e^{2 \pi i \theta t}\right\}_{t \in[0,2 \pi]}$. Here $e^{2 \pi i \theta t}$ is regarded as an element of $\operatorname{Sp}(2)$ via the inclusion $\mathrm{U}(1)=$ $\mathrm{SO}(2) \subset \mathrm{Sp}(2)$. A homotopy as just described can be used to define a continuous path of Fredholm operators, and hence an isomorphism

$$
\mathcal{O}\left(\widetilde{C}_{S}\right) \simeq \mathcal{O}\left(\widetilde{C}_{\theta}\right)
$$

via (9.4). Moreover, for any given $\theta \in \mathbb{R} \backslash \mathbb{Z}$, the set of families $\{S(t)\}$ satisfying $\left({ }^{*}\right)$ is contractible, so the isomorphism (9.32) is canonical. Thus the canonical orientation in $\mathcal{O}\left(\widetilde{C}_{\theta}\right)$ induces a canonical orientation in $\mathcal{O}\left(\widetilde{C}_{S}\right)$. Our last axiom is now:

(OR4) If $\{S(t)\}_{t \in S^{1}}$ is a family of $2 \times 2$ symmetric matrices such that the associated $2 \times 2$ symplectic matrix $\Phi(2 \pi)$ is elliptic, then $\mathfrak{o}_{{ }}{ }_{S}$ is the canonical choice described above.

Remark 9.10. Axioms (OR1) to (OR4) imply the following generalization of (OR4). Consider an orientation triple $\widetilde{C}=\left(C, E,\left\{S_{k}\right\}\right)$ such that each end is elliptic, i.e., for each $k$, if $\left\{\Phi_{k}(t)\right\}_{t \in[0,2 \pi]}$ is the path of symplectic matrices determined by $S_{k}$, then $\Phi_{k}(2 \pi)$ has eigenvalues on the unit circle. Then there is a canonical orientation in $\mathcal{O}(\widetilde{C})$, obtained by deforming to the complex linear case as in (9.32). (For example, this is how we orient the operator $D_{\Sigma}$ and with it the obstruction bundle $\mathcal{O}$, in Section I.2.6.) Axioms (OR1) to (OR4) imply that $\mathfrak{o}_{\widetilde{C}}$ agrees with this canonical orientation.

\section{Counting ends of the index 2 moduli space}

Let $\left(U_{+}, U_{-}\right)$be a gluing pair satisfying (1.1) and (1.2). Throughout this section, fix $h<\lambda / 4 \Lambda$ in the gluing construction as in Section 8.2, and fix a system of coherent orientations. Also assume that $J$ is generic so that all non-multiply-covered $J$-holomorphic curves are unobstructed, all nonmultiply-covered $J$-holomorphic curves of index $\leq 2$ are immersed (see Theorem 4.1), the obstruction section $\mathfrak{s}$ vanishes only for simple branched covers (see Lemma 6.4) where it is smooth (by Lemma 6.3) and the collection of eigenfunctions $\gamma$ in Section 8.1 is admissible (see Propositions 3.2 and 3.9).

Recall that $\alpha_{+}$denotes the list of Reeb orbits corresponding to the positive ends of $U_{+}$, and $\alpha_{-}$denotes the list of Reeb orbits corresponding to the negative ends of $U_{-}$. This section will relate the count of zeroes of $\mathfrak{s}$ defined in Section 8.2, to a count of those ends of the index 2 part of the moduli space $\mathcal{M}^{J}\left(\alpha_{+}, \alpha_{-}\right) / \mathbb{R}$ that are "close to breaking" into $U_{+}$and $U_{-}$along branched covers of $\mathbb{R} \times \alpha$. This entails putting together the previous results, and then comparing signs of zeroes of $\mathfrak{s}$ with signs associated to these ends via the coherent orientations. 
10.1. Statement of the result. Recall the definition of $\mathcal{G}_{\delta}\left(U_{+}, U_{-}\right)$from Section 7.1; this describes curves that are "close to breaking" in the above sense. In Section I.1.3 we defined an integer $\# G\left(U_{+}, U_{-}\right)$which counts ends of the index 2 moduli space in $\mathcal{G}_{\delta}\left(U_{+}, U_{-}\right) / \mathbb{R}$. We recall the definition here for convenience:

Definition 10.1. Let $0<\delta^{\prime}<\delta$ be small, and let $\mathcal{U} \subset \mathcal{M}^{J}\left(\alpha_{+} . \alpha_{-}\right) / \mathbb{R}$ be an open set such that:

- $\mathcal{G}_{\delta^{\prime}}\left(U_{+}, U_{-}\right) / \mathbb{R} \subset \mathcal{U} \subset \mathcal{G}_{\delta}\left(U_{+}, U_{-}\right) / \mathbb{R}$.

- The closure $\overline{\mathcal{U}}$ has only finitely many boundary points.

Define $\# G\left(U_{+}, U_{-}\right) \in \mathbb{Z}$ to be minus the signed count of boundary points of $\overline{\mathcal{U}}$, where $\overline{\mathcal{U}}$ is oriented via the coherent orientations. (The orientation on $\mathcal{M}^{J} / \mathbb{R}$ is induced from that of $\mathcal{M}^{J}$ via the " $\mathbb{R}$-direction first" convention, see Section I.1.1.) Lemma I.1.11 implies that if $\delta>0$ is sufficiently small, then this count is well defined and independent of choices.

The main result of this section is the following theorem, which relates $\# G\left(U_{+}, U_{-}\right)$to the count of zeroes of $\mathfrak{s}$ that was defined in Section 8.2.

Theorem 10.2. In the gluing construction, if we choose $r$ sufficiently large, then for $R$ sufficiently large,

$$
\# G\left(U_{+}, U_{-}\right)=\epsilon\left(U_{+}\right) \cdot \epsilon\left(U_{-}\right) \cdot \#\left(\mathfrak{s}^{-1}(0) \cap \mathcal{V}_{R} / \mathbb{R}\right) .
$$

Here $\epsilon\left(U_{ \pm}\right)$denotes the sign associated to $U_{ \pm}$by the system of coherent orientations. Theorem 10.2, together with Corollary 8.6 (see Remark 8.5), implies the main Theorem 1.1.

10.2. Reducing to a local statement. We now use the gluing theorem 7.3 to reduce Theorem 10.2 to a "local" statement involving comparing orientations.

Recall from Theorem 7.3(b) that if $\delta>0$ is sufficiently small with respect to $r$, then the gluing map identifies $\mathcal{G}_{\delta}\left(U_{+}, U_{-}\right)$with a subset of $\mathfrak{s}^{-1}(0)$. Moreover, by Theorem 7.3(a) and Proposition 8.2, if $R$ is sufficiently large, then the gluing map sends $\mathfrak{s}^{-1}(0) \cap \mathcal{V}_{R}$ into $\mathcal{G}_{\delta}\left(U_{+}, U_{-}\right)$. For such $R$, our fixed coherent orientations determine an orientation of the 1 -manifold $\mathfrak{s}^{-1}(0) / \mathbb{R}$ in a neighborhood of $\mathcal{V}_{R} / \mathbb{R}$.

We will see in Section 10.3 and Section 10.5 that our assumptions on $J$ imply that $\mathfrak{s}$ is transverse to the zero section, so that $\mathfrak{s}^{-1}(0)$ is smooth. Choose $R$ large as above, and generic so that $\mathfrak{s}^{-1}(0) / \mathbb{R}$ intersects $\mathcal{V}_{R} / \mathbb{R}$ transversely in a finite set of points. For each point

$$
\left(T_{-}, T_{+},[\Sigma]\right) \in \mathfrak{s}^{-1}(0) \cap \mathcal{V}_{R} / \mathbb{R},
$$

define a sign

$$
\epsilon_{\mathcal{M}}\left(T_{-}, T_{+},[\Sigma]\right) \in\{ \pm 1\}
$$


as follows: $\epsilon_{\mathcal{M}}\left(T_{-}, T_{+},[\Sigma]\right):=+1$ if and only if near $\left(T_{-}, T_{+},[\Sigma]\right)$, the orientation on $\mathfrak{s}^{-1}(0) / \mathbb{R}$ determined by the coherent orientations points in the increasing $R$ direction. Also, define

$$
\epsilon_{\mathfrak{s}}\left(T_{-}, T_{+},[\Sigma]\right) \in\{ \pm 1\}
$$

to be the sign of $\left(T_{-}, T_{+},[\Sigma]\right)$ as a zero of $\mathfrak{s}$, see Section 8.2.

Lemma 10.3. If $r$ is chosen sufficiently large in the gluing construction, and if $R$ is sufficiently large with respect to $r$, and generic so that $\mathfrak{s}^{-1}(0)$ intersects $\mathcal{V}_{R}$ transversely, then

$$
\# G\left(U_{+}, U_{-}\right)=\sum_{\left(T_{-}, T_{+},[\Sigma]\right) \in \mathfrak{s}^{-1}(0) \cap \mathcal{V}_{R} / \mathbb{R}} \epsilon_{\mathcal{M}}\left(T_{-}, T_{+},[\Sigma]\right) .
$$

Proof. Fix $r>r_{0}$ sufficiently large, and $\delta>0$ sufficiently small with respect to $r$, as in Theorem 7.3(b). By Theorem 7.3(a) and Proposition 8.2, if $R$ is sufficiently large, then the gluing map sends $\mathfrak{s}^{-1}(0) \cap \bigcup_{R^{\prime} \geq R} \mathcal{V}_{R^{\prime}}$ into $\mathcal{G}_{\delta}\left(U_{+}, U_{-}\right)$. Fix a generic such $R$ so that $\mathfrak{s}^{-1}(0)$ intersects $\mathcal{V}_{R}$ transversely. Define $\mathcal{U} \subset \mathcal{M}^{J}\left(\alpha_{+}, \alpha_{-}\right) / \mathbb{R}$ to be the image of the gluing map on $\mathfrak{s}^{-1}(0) \cap$ $\bigcup_{R^{\prime}>R} \mathcal{V}_{R^{\prime}}$. By Theorem 7.3(b) and the compactness of the set $\bigcup_{R^{\prime} \leq R} \mathcal{V}_{R} / \mathbb{R}$, there exists $\delta^{\prime} \in(0, \delta)$ such that $\mathcal{U}$ contains all of $\mathcal{G}_{\delta^{\prime}}\left(U_{+}, U_{-}\right) / \overline{\mathbb{R}}$. As in Definition 10.1, if $\delta$ is sufficiently small (the $\delta$ chosen above is already small enough), then $\# G\left(U_{+}, U_{-}\right)=-\# \partial \overline{\mathcal{U}}$. But $-\# \partial \overline{\mathcal{U}}$ is clearly the same as the count on the right hand side of (10.1).

As a consequence, to prove Theorem 10.2, it sufficies to prove the following:

Lemma 10.4. If $r$ is chosen sufficiently large in the gluing construction, then with $R$ as in Lemma 10.3, for each $\left(T_{-}, T_{+},[\Sigma]\right) \in \mathfrak{s}^{-1}(0) \cap \mathcal{V}_{R} / \mathbb{R}$, we have

$$
\epsilon_{\mathcal{M}}\left(T_{-}, T_{+},[\Sigma]\right)=\epsilon\left(U_{-}\right) \cdot \epsilon\left(U_{+}\right) \cdot \epsilon_{\mathfrak{s}}\left(T_{-}, T_{+},[\Sigma]\right)
$$

10.3. Eliminating the coherent orientations. We now reduce Lemma 10.4 to a more explicit statement which does not refer to coherent orientations.

Recall from Section 5.8 that the obstruction section $\mathfrak{s}$ is defined on the set of triples $\left(T_{-}, T_{+}, \Sigma\right)$ with $T_{+} \geq 5 r$ and $\Sigma \in \mathcal{M}$. It proves convenient henceforth to replace the coordinates $\left(T_{-}, T_{+}\right)$by

$$
R_{-}:=s_{-}-T_{-}, \quad R_{+}:=s_{+}+T_{+} .
$$

That is, $R_{ \pm}$is the amount by which the curve $u_{ \pm}$is translated in the $\mathbb{R}$ direction in the pregluing. In these coordinates, $\mathfrak{s}$ is defined on triples $\left(R_{-}, R_{+}, \Sigma\right)$ with $R_{+} \geq s_{+}+5 r$ and $R_{-} \geq s_{-}-5 r$. The set $\mathcal{V}_{R}$ in Section 8.2 corresponds to triples $\left(R_{-}, R_{+}, \Sigma\right)$ as above such that $R_{+}-R_{-}=R$. Any element of $\mathcal{V}_{R} / \mathbb{R}$ has a distinguished representative with $R_{ \pm}= \pm R / 2$, and this determines the identification of $\mathcal{V}_{R} / \mathbb{R}$ with a subset of $\mathcal{M}$ from Section 8.2. 
In these new coordinates, consider a point $\left(R_{-}, R_{+}, \Sigma\right) \in \mathfrak{s}^{-1}(0) \cap \mathcal{V}_{R}$, with $R$ large as in Lemma 10.3, and let $C$ denote the corresponding $J$ holomorphic curve given by the gluing theorem. The key to the proof of Lemma 10.4 is to compare signs associated to (i) the derivative of the gluing construction and (ii) linear gluing.

(i) Use the $L^{2}$ inner product to identify $\operatorname{Coker}\left(D_{\Sigma}\right)$ with its dual, and thereby regard $\mathfrak{s}$ as taking values in $\operatorname{Coker}\left(D_{\Sigma}\right)$. We then have a sequence of maps

$$
0 \longrightarrow \operatorname{Ker}\left(D_{C}\right) \stackrel{\mathcal{I}}{\longrightarrow} \mathbb{R}^{2} \oplus T_{\Sigma} \mathcal{M} \stackrel{\nabla \mathfrak{s}}{\longrightarrow} \operatorname{Coker}\left(D_{\Sigma}\right) \longrightarrow 0 .
$$

Here $\nabla \mathfrak{s}$ denotes the differential of $\mathfrak{s}$ at $\left(R_{-}, R_{+}, \Sigma\right)$. Meanwhile,

$$
\mathcal{I}: \operatorname{Ker}\left(D_{C}\right) \stackrel{\simeq}{\longrightarrow} \operatorname{Ker}(\nabla \mathfrak{s})
$$

is the inverse of the derivative of the gluing map. (The discussion in Section 6.3 shows that the gluing map is smooth here, and we will see in Section 10.5 that its derivative $\operatorname{Ker}(\nabla \mathfrak{s}) \rightarrow \operatorname{Ker}\left(D_{C}\right)$ is an isomorphism.) Since $C$ is unobstructed, dimension counting shows that the sequence (10.2) is exact. Since $T_{\Sigma} \mathcal{M}$ and $\operatorname{Coker}\left(D_{\Sigma}\right)$ have canonical orientations, the exact sequence (10.2) determines an isomorphism

$$
\Phi_{1}: \mathcal{O}\left(\operatorname{Ker}\left(D_{C}\right)\right) \simeq \mathcal{O}\left(\mathbb{R}^{2}\right) .
$$

(ii) The linear gluing construction from Section 9 defines an isomorphism

$$
\mathcal{O}\left(D_{C}\right) \simeq \mathcal{O}\left(D_{-}\right) \otimes \mathcal{O}\left(D_{0}\right) \otimes \mathcal{O}\left(D_{+}\right)
$$

where $D_{0}$ is an appropriate index 0 operator on $\Sigma$, compare Section 10.4. By Remark 9.10, there is a canonical orientation in $\mathcal{O}\left(D_{0}\right)$. Thus, since $D_{ \pm}$and $D_{C}$ are unobstructed, the isomorphism (10.4) determines an isomorphism

$$
\mathcal{O}\left(\operatorname{Ker}\left(D_{C}\right)\right) \simeq \mathcal{O}\left(\operatorname{Ker}\left(D_{-}\right)\right) \otimes \mathcal{O}\left(\operatorname{Ker}\left(D_{+}\right)\right)
$$

On the other hand, the $\mathbb{R}$-action on the moduli spaces of $J$-holomorphic curves determines isomorphisms $\operatorname{Ker}\left(D_{ \pm}\right) \simeq \mathbb{R}$. Thus the isomorphism (10.5) determines an isomorphism

$$
\Phi_{2}: \mathcal{O}\left(\operatorname{Ker}\left(D_{C}\right)\right) \simeq \mathcal{O}\left(\mathbb{R}^{2}\right) .
$$

Lemma 10.5. If $r$ is sufficiently large in the gluing construction, and if $R$ is sufficiently large as in Lemma 10.3, then for each $\left(R_{-}, R_{+}, \Sigma\right) \in \mathfrak{s}^{-1}(0) \cap \mathcal{V}_{R}$, the isomorphisms $\Phi_{1}$ and $\Phi_{2}$ defined in (10.3) and (10.6) agree.

This lemma will be proved in Section 10.5. Granted this, we can now give:

Proof of Lemma 10.4. Assume that $r$ is sufficiently large as in Lemma 10.5 and that $R$ is sufficiently large as in Lemma 10.3 , and let $\left(R_{-}, R_{+}, \Sigma\right)$ be a 
transverse intersection of $\mathfrak{s}^{-1}(0)$ with $\mathcal{V}_{R}$. Transversality here means that the projection

$$
\Pi: T_{\left(R_{-}, R_{+}, \Sigma\right) \mathfrak{s}^{-1}}(0) \longrightarrow T_{\left(R_{-}, R_{+}\right)} \mathbb{R}^{2}
$$

is an isomorphism. Moreover,

$$
\epsilon_{\mathcal{M}}\left(R_{-}, R_{+}, \Sigma\right)=\operatorname{sign}(\operatorname{det}(\Pi))
$$

where $\operatorname{sign}(\operatorname{det}(\Pi))$ is computed using the orientation of $T_{\left(R_{-}, R_{+}, \Sigma\right)} \mathfrak{s}^{-1}(0)$ determined by the coherent orientations, together with the standard orientation of $\mathbb{R}^{2}$. Using these same orientations, we also see from the exact sequence (10.2) that

$$
\operatorname{sign}\left(\Phi_{1}\right)=\operatorname{sign}(\operatorname{det}(\Pi)) \cdot \epsilon_{\mathfrak{s}}\left(R_{-}, R_{+}, \Sigma\right) .
$$

On the other hand, we have

$$
\operatorname{sign}\left(\Phi_{2}\right)=\epsilon\left(U_{-}\right) \cdot \epsilon\left(U_{+}\right),
$$

because by definition the isomorphism $\mathcal{O}\left(D_{ \pm}\right) \simeq \mathbb{R}$ is orientation-preserving if and only if $\epsilon\left(U_{ \pm}\right)= \pm 1$. Combining the above three equations with Lemma 10.5 proves Lemma 10.4.

10.4. Setting up the linear gluing exact sequence. Fix $\left(R_{-}, R_{+}, \Sigma\right) \in$ $\mathfrak{s}^{-1}(0)$, and let $C$ denote the associated glued curve. To prepare for the proof of Lemma 10.5, we now show that if $r, T_{-}, T_{+}$are sufficiently large, then a version of the linear gluing exact sequence (9.30) is applicable, with $C_{ \pm}=u_{ \pm}$and $C_{0} \approx \Sigma$.

Here is the precise setup. Let $E_{ \pm}$denote the normal bundle to $u_{ \pm}$, and let $E_{C}$ denote the normal bundle to $C$. Recall that we have linear deformation operators $D_{ \pm}: C^{\infty}\left(E_{ \pm}\right) \rightarrow C^{\infty}\left(T^{0,1} C_{ \pm} \otimes E_{ \pm}\right)$and $D_{C}: C^{\infty}\left(E_{C}\right) \rightarrow$ $C^{\infty}\left(T^{0,1} C \otimes E_{C}\right)$. Use the coordinates $z, w$ in a neighborhood of the Reeb orbit $\alpha$ as usual to trivialize $E_{-}$over the positive ends of $C_{-}$and to trivialize $E_{+}$over the negative ends of $C_{+}$.

Let $C^{\prime}$ denote the surface obtained from $C$ by removing the $s<s_{-}-T_{-}$ portion of the first $N_{-}$negative ends and the $s>s_{+}+T_{+}$portion of the first $N_{+}$positive ends. Let $C_{0}$ denote the surface obtained from $C^{\prime}$ by attaching infinite cylindrical ends to the boundary circles. Note that $C_{0}$ is naturally identified with $\Sigma$, because $\Sigma^{\prime}$ parameterizes $C^{\prime}$ by a map sending $u \mapsto(z, w)=\left(\pi(u), \psi_{\Sigma}(u)\right)$. The identification $\imath: C_{0} \rightarrow \Sigma$ is bi-Lipschitz, and off of the ramification points it is smooth.

For future reference, here is a more explicit description of $C_{0}$ near a ramification point $p$. Let $v$ be a holomorphic local coordinate on $C_{0}$ that vanishes at $p$. It follows from (2.1), as in Section 6.4, that the holomorphic 
coordinate $v$ can be rescaled so that near $v=0$,

$$
\begin{aligned}
z & =z_{0}+\left(1-\left|a_{0}\right|^{2}\right)^{-1}\left(v^{2}+a_{0} \bar{v}^{2}\right)+O\left(|v|^{3}\right), \\
w & =w_{0}+c v+O\left(|v|^{2}\right),
\end{aligned}
$$

where $z_{0}, w_{0}, a_{0}$ denote the values of $z, w, a$ at $p$, and $c$ is a nonzero constant.

Now define a bundle $E_{0}$ over $C_{0}$, and a differential operator

$$
D_{0}: C^{\infty}\left(E_{0}\right) \rightarrow C^{\infty}\left(T^{0,1} C_{0} \otimes E_{0}\right),
$$

as follows. Over $C^{\prime}$, define $E_{0}$ to be the pullback of the normal bundle to $C$ in $\mathbb{R} \times Y$. The coordinate $w$ trivializes this bundle on the complement of the ramification points. Use this trivialization to extend $E_{0}$, with trivialization, over the ends of $C_{0}$.

Next, define a continuous bundle map

$$
\imath^{0,1}: T^{0,1} \Sigma \longrightarrow T^{0,1} C_{0}
$$

as follows. On the complement of the ramification points, $\imath^{0,1}$ is a smooth bundle map defined by pulling back from $T^{0,1} \Sigma$ to $T_{\mathbb{C}}^{*} C_{0}$ via the map $\imath$ : $C_{0} \rightarrow \Sigma$, and then projecting along $T^{1,0} C_{0}$ to $T^{0,1} C_{0}$. Here the complex structure on $C_{0}$ is chosen to agree with that of $C$ over the support of $\beta_{\Sigma}$ and to agree with the standard complex structure on the cylinder over the ends of $C_{0}$. The map (10.8) extends continuously over the ramification points, where it is zero. We can choose the complex structure on $C_{0}$ so that $\imath^{0,1}$ is an isomorphism on the complement of the ramification points; this is because the $(0,1)$ part of the 1 -form $d \bar{z}$ is

$$
(d \bar{z})^{0,1}=\left(1-|a|^{2}\right)^{-1}(d \bar{z}-\bar{a} d z) .
$$

Finally, define

$$
D_{0}:=\beta_{\Sigma} D_{C}+\left(1-\beta_{\Sigma}\right) \imath^{0,1} D_{\Sigma},
$$

where $\beta_{\Sigma}$ is the cutoff function defined in Section 5.3.

In order to obtain a version of the linear gluing exact sequence, we want to choose a finite dimensional subspace $V_{0} \subset L^{2}\left(T^{0,1} C_{0} \otimes E_{0}\right)$ such that if $W_{0}$ denotes the orthogonal complement of $V_{0}$ and if $\Pi_{W_{0}}$ denotes the orthogonal projection onto $W_{0}$, then $\Pi_{W_{0}} D_{0}$ is surjective. For this purpose, define a continuous bundle map

$$
\mathfrak{n}: \Sigma \times \mathbb{C} \longrightarrow E_{0},
$$

covering the Lipschitz map $\imath^{-1}: \Sigma \rightarrow C_{0}$, as follows. Over $\Sigma \backslash \Sigma^{\prime}$, the map $\mathfrak{n}$ is just the trivialization of $E_{0}$ over the ends of $C_{0}$. Given $u \in \Sigma^{\prime}$ and $w \in \mathbb{C}$, let $p$ denote the point in $C$ corresponding to $u$, let $W \in T_{p}(\mathbb{R} \times Y)$ denote the tangent vector to the $z=$ constant disc corresponding to $w$ and define $\mathfrak{n}(u, w)$ to be the projection of $W$ onto the normal bundle to $C$ at $p$. Note that $\mathfrak{n}$ is zero at each ramification point and an isomorphism at every 
other point of $\Sigma$. Tensoring the bundle maps (10.8) and (10.11) defines a Lipschitz bundle map

$$
\mathfrak{n}^{0,1}:=\imath^{0,1} \otimes \mathfrak{n}: T^{0,1} \Sigma \longrightarrow T^{0,1} C_{0} \otimes E_{0}
$$

which vanishes at the ramification points and is a smooth isomorphism elsewhere. In terms of this last bundle map, define

$$
V_{0}:=\mathfrak{n}^{0,1}\left(\operatorname{Coker}\left(D_{\Sigma}\right)\right) \subset L^{2}\left(T^{0,1} C_{0} \otimes E_{0}\right) .
$$

We will see below that $\Pi_{W_{0}} D_{0}$ is surjective for this choice of $V_{0}$. We also want to understand the kernel of $\Pi_{W_{0}} D_{0}$. For this purpose, define a linear map

$$
\rho: C^{0}\left(E_{0}\right) \longrightarrow T_{\Sigma} \mathcal{M}
$$

as follows. Let $\mathcal{R}$ denote the set of ramification points of $\Sigma$. Due to our choice of almost complex structure $J$, the ramification points are simple, so branched covers in $\mathcal{M}$ near $\Sigma$ are determined by the projections of their ramification points to $\mathbb{R} \times S^{1}$. Consequently, there is a natural identification

$$
T_{\Sigma} \mathcal{M}=\bigoplus_{u \in \mathcal{R}} T_{\pi(u)}\left(\mathbb{R} \times S^{1}\right)
$$

Under this identification, the map $\rho$ sends a continuous section $\psi$ of $E_{0}$ to the collection of tangent vectors $\left(z_{u}\right)_{u \in \mathcal{R}}$, where $z_{u}$ denotes the pushforward of $\psi(u)$ by the projection $(z, w) \mapsto(z, 0)$.

Lemma 10.6. If $r$ is sufficiently large in the gluing construction, then

(a) $\Pi_{W_{0}} D_{0}: L_{1}^{2}\left(E_{0}\right) \rightarrow W_{0}$ is surjective.

(b) The map $\rho$ restricts to an isomorphism

$$
\rho: \operatorname{Ker}\left(\Pi_{W_{0}} D_{0}\right) \stackrel{\simeq}{\longrightarrow} T_{\Sigma} \mathcal{M}
$$

Proof. First note that if $\psi \in \operatorname{Ker}\left(\Pi_{W_{0}} D_{0}\right)$, then by definition there exists $\eta \in \operatorname{Coker}\left(D_{\Sigma}\right)$ with $D_{0} \psi=\mathfrak{n}^{0,1} \eta$. Since $\eta$ is a smooth $(0,1)$-form on $\Sigma$, and since $\mathfrak{n}^{0,1}$ is Lipschitz, it follows that $D_{0} \psi$ has bounded first derivatives near the ramification points, and so by elliptic regularity $\psi$ is $C^{1}$. In particular, $\rho$ is well defined on $\operatorname{Ker}\left(\Pi_{W_{0}} D_{0}\right)$.

Now since the index of the operator $\Pi_{W_{0}} D_{0}$ equals the dimension of $T_{\Sigma} \mathcal{M}$, to prove both (a) and (b) it suffices to show that the map (10.12) is injective. Suppose $\psi \in \operatorname{Ker}\left(\Pi_{W_{0}} D_{0}\right)$ satisfies $\rho(\psi)=0$; we will show that $\psi=0$.

Step 1. We first show that there exists $\zeta \in L_{1}^{2}(\Sigma, \mathbb{C})$ with $\mathfrak{n} \zeta=\psi$.

The only issue is to check that $\mathfrak{n}^{-1} \psi$ is $L_{1}^{2}$ in a neighborhood of each ramification point $p$. Near $p$, in terms of the local description (10.7), 
the normal bundle to $C_{0}$ near $v=0$ is trivialized by a $(1,0)$-form that annihilates $T C_{0}$ and has the form

(10.13)

$$
\widehat{n}=\frac{c}{2}\left(d z-a_{0} d \bar{z}\right)-v d w+O(|v|) d z+O(|v|) d \bar{z}+O\left(|v|^{2}\right) d w+O\left(|v|^{2}\right) d \bar{w} .
$$

It follows that in this local trivialization, using the Lipschitz identification $\imath: \Sigma \rightarrow C_{0}$ to regard $\mathfrak{n}$ as a map between bundles over $C_{0}$, we have

$$
\mathfrak{n}=-v+O\left(|v|^{2}\right) \text {. }
$$

Since $D_{0} \psi \in V_{0}$, we know that $\psi$ is $C^{1}$ and $D_{0} \psi=O(|v|)$, and since $\rho(\psi)=0$ we know that $\psi=O(|v|)$. It follows that in the local trivialization (10.13),

$$
\frac{\partial \psi}{\partial \bar{v}}=O(|v|) .
$$

Now (10.14) and (10.15) imply that $\partial\left(\mathfrak{n}^{-1} \psi\right) / \partial \bar{v}$ is bounded, and so by elliptic regularity again, $\mathfrak{n}^{-1} \psi$ is $L_{1}^{2}$.

Step 2. We now show that if $\zeta \in L_{1}^{2}(\Sigma, \mathbb{C})$ satisfies $\Pi_{W_{0}} D_{0}(\mathfrak{n} \zeta)=0$, then $\zeta=0$.

We begin by deriving a useful formula for $D_{0}(\mathfrak{n} \zeta)$. First restrict attention to $\Sigma^{\prime}$. Here, off of the ramification points, regard $\psi_{\Sigma}$ locally as a function of $z \in \mathbb{R} \times S^{1}$. Recall from Section 3.1 that the graph, $C$, of $\psi_{\Sigma}$ is $J$ holomorphic if and only if $\bar{\partial}(C)=0$, where $\bar{\partial}(C)$ denotes the 1-form on $C$ with values in the normal bundle $N_{C}$ that inputs a tangent vector $v$ and outputs the projection of $J v$ to $N_{C}$. To describe $\bar{\partial}(C)$ more explicitly in the present case, note that the projection from $\left.T(\mathbb{R} \times Y)\right|_{C}$ to $N_{C}$ is given by the composition of $\mathfrak{n}$ with the 1 -form $d w-d \psi_{\Sigma}$. Hence. $\mathfrak{n}^{-1} \bar{\partial}(C)$ is the restriction to $C$ of $-2 i$ times the $(0,1)$ part of $d w-d \psi_{\Sigma}$. By $(2.1)$ and (10.9), this gives

$$
\bar{\partial}(C)=2 i \mathfrak{n}^{0,1}\left(\left(\frac{\partial \psi_{\Sigma}}{\partial \bar{z}}+a \frac{\partial \psi_{\Sigma}}{\partial z}+b\right) d \bar{z}\right) .
$$

By definition,

$$
D_{C}(\mathfrak{n} \zeta)=\left.\frac{1}{2 i} \frac{d}{d \epsilon}\right|_{\epsilon=0} \bar{\partial}\left(C_{\epsilon}\right)
$$

where $C_{\epsilon}$ denotes the graph of $\psi_{\Sigma}+\epsilon \zeta$. Therefore,

$$
\begin{aligned}
D_{C}(\mathfrak{n} \zeta) & =\mathfrak{n}^{0,1}\left(\left(\frac{\partial \zeta}{\partial \bar{z}}+a \frac{\partial \zeta}{\partial z}+\left(\nabla_{\zeta} a\right) \frac{\partial \psi_{\Sigma}}{\partial z}+\nabla_{\zeta} b\right) d \bar{z}\right) \\
& =\mathfrak{n}^{0,1}\left(D_{\Sigma} \zeta+\mathcal{R}(\zeta)\right)
\end{aligned}
$$

where $\nabla_{\zeta}$ denotes the derivative along the $z=$ constant disks in the direction determined by $\zeta$, and

$$
\mathcal{R}(\zeta):=\left(a \frac{\partial \zeta}{\partial z}+\left(\nabla_{\zeta} a\right) \frac{\partial \psi_{\Sigma}}{\partial z}+\left(\nabla_{\zeta} b-\left.\nabla_{\zeta} b\right|_{w=0}\right)\right) d \bar{z} .
$$


It then follows from (10.10) that on all of $\Sigma$,

$$
D_{0}(\mathfrak{n} \zeta)=\mathfrak{n}^{0,1}\left(D_{\Sigma} \zeta+\beta_{\Sigma} \mathcal{R}(\zeta)\right)
$$

To use this formula, note that our assumption that $\Pi_{W_{0}} D_{0}(\mathfrak{n} \zeta)=0$ means that $D_{0}(\mathfrak{n} \zeta) \in \mathfrak{n}^{0,1} \operatorname{Coker}\left(D_{\Sigma}\right)$. Since $\mathfrak{n}^{0,1}$ is an isomorphism except at the ramification points, it follows from this and (10.19) that

$$
D_{\Sigma} \zeta+\beta_{\Sigma} \mathcal{R}(\zeta) \in \operatorname{Coker}\left(D_{\Sigma}\right)
$$

Now recall from the proof of Lemma 5.5 that there is a constant $c>0$, not depending on $\Sigma, T_{-}, T_{+}$, such that

$$
\left\|D_{\Sigma} \zeta\right\|_{L^{2}} \geq c\|\zeta\|_{L_{1}^{2}}
$$

On the other hand, inspection of (10.18) shows that there is a constant $c^{\prime}$ with

$$
|\mathcal{R}(\zeta)| \leq c^{\prime}|w|(|\zeta|+|\nabla \zeta|)
$$

It follows from (10.20), (10.21), and (10.22) that if $r$ is sufficiently large, so that $|w|$ is always sufficiently small on the support of $\beta_{\Sigma}$, then $\zeta=0$.

10.5. A homotopy of exact sequences. With all the setup in place, we come now to the heart of the proof of Theorem 10.2. If $r$ is sufficiently large, then since $D_{ \pm}, D_{C}$ and $\Pi_{W_{0}} D_{0}$ are surjective, as in (9.30), we obtain an exact sequence

$$
0 \longrightarrow \operatorname{Ker}\left(D_{C}\right) \stackrel{f}{\longrightarrow} \operatorname{Ker}\left(D_{-}\right) \oplus \operatorname{Ker}\left(\Pi_{W_{0}} D_{0}\right) \oplus \operatorname{Ker}\left(D_{+}\right) \stackrel{g}{\longrightarrow} V_{0} \longrightarrow 0 .
$$

Here we use the cutoff functions $\beta_{-}, \beta_{0}:=\beta_{\Sigma}$ and $\beta_{+}$from Section 5 ; these are slightly different from the cutoff functions in (9.30), but the resulting exact sequence will be the same up to homotopy, as in Section 9.3. Moreover, the isomorphisms

$$
\operatorname{Coker}\left(D_{\Sigma}\right) \simeq V_{0}, \quad \operatorname{Ker}\left(\Pi_{W_{0}} D_{0}\right) \simeq T_{\Sigma} \mathcal{M}, \quad \operatorname{Ker}\left(D_{ \pm}\right) \simeq \mathbb{R},
$$

determined by $\mathfrak{n}^{0,1}, \rho$ and the $\mathbb{R}$-action, respectively, identify the terms in the exact sequence (10.23) with those in the exact sequence (10.2), although the maps may be different. (In these identifications, we are commuting $\operatorname{Ker}\left(D_{+}\right)$with $\operatorname{Ker}\left(\Pi_{W_{0}} D_{0}\right)$, which has no effect on orientations since the latter is even dimensional.) On the other hand, the exact sequence (10.23), together with the exact sequence

$$
0 \longrightarrow \operatorname{Ker}\left(D_{0}\right) \longrightarrow \operatorname{Ker}\left(\Pi_{W_{0}} D_{0}\right) \stackrel{D_{0}}{\longrightarrow} V_{0} \longrightarrow \operatorname{Coker}\left(D_{0}\right) \longrightarrow 0
$$

determines the isomorphism (10.4) on orientations. So to prove Lemma 10.5, and thus Theorem 10.2, it is enough to prove the following: 
Lemma 10.7. Let $\left(R_{-}, R_{+}, \Sigma\right) \in \mathfrak{s}^{-1}(0)$. If $r$ is sufficiently large in the gluing construction, then under the identifications (10.24):

(a) The isomorphism

$$
\mathcal{O}\left(T_{\Sigma} \mathcal{M}\right) \otimes \mathcal{O}\left(\operatorname{Coker}\left(D_{\Sigma}\right)\right) \stackrel{\simeq}{\longrightarrow} \mathcal{O}\left(D_{0}\right)
$$

determined by (10.25) sends the tensor product of the canonical orientations of $T_{\Sigma} \mathcal{M}$ and $\operatorname{Coker}\left(D_{\Sigma}\right)$ to the canonical orientation of $D_{0}$.

(b) The exact sequences (10.2) and (10.23) are homotopic through exact sequences, and so induce the same isomorphism on orientations.

Proof. Assertion (a) follows by deforming to the complex linear case.

The proof of assertion (b) has the following outline:

Part 1. We will first construct a homotopy of exact sequences

$$
0 \longrightarrow \operatorname{Ker}\left(D_{C}\right) \stackrel{f_{\tau}}{\longrightarrow} \mathbb{R}^{2} \oplus T_{\Sigma} \mathcal{M} \stackrel{g_{\tau}}{\longrightarrow} \operatorname{Coker}\left(D_{\Sigma}\right) \longrightarrow 0
$$

parameterized by $\tau \in[0,1]$, such that when $\tau=1$, the exact sequence (10.26) agrees with (10.2).

Part 2. We will then relate the exact sequence (10.26) for $\tau=0$ to the exact sequence (10.23) by defining a map

$$
\widetilde{\rho}: \operatorname{Ker}\left(D_{-}\right) \oplus \operatorname{Ker}\left(\Pi_{W_{0}} D_{0}\right) \oplus \operatorname{Ker}\left(D_{+}\right) \stackrel{\simeq}{\longrightarrow} \mathbb{R}^{2} \oplus T_{\Sigma} \mathcal{M}
$$

such that the following diagram commutes:

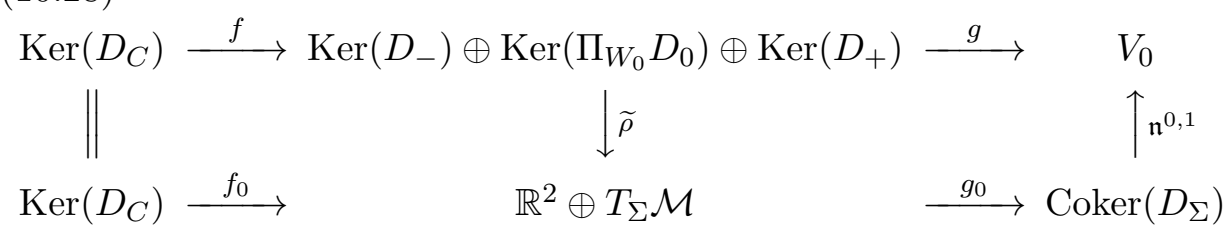

Part 3. Lastly, we will show that $\widetilde{\rho}$ is an isomorphism which is homotopic through isomorphisms to the isomorphism given by $\rho$ and the identifications $\operatorname{Ker}\left(D_{ \pm}\right) \simeq \mathbb{R}$

The details follow.

Part 1. Fix $\left(r_{-}, r_{+}, X\right) \in \mathbb{R}^{2} \oplus T_{\Sigma} \mathcal{M}$, and fix $\tau \in[0,1]$. We begin with a somewhat lengthy definition of $g_{\tau}\left(r_{-}, r_{+}, X\right)$.

Let $\phi_{ \pm} \in \operatorname{Ker}\left(D_{ \pm}\right)$correspond to $r_{ \pm} \in \mathbb{R}$. Let $\phi_{0}$ denote the unique element of $\operatorname{Ker}\left(\Pi_{W_{0}} D_{0}\right)$ for which $\rho\left(\phi_{0}\right)=X$; this is provided by Lemma 10.6. Given $\zeta_{ \pm} \in L_{1}^{2}\left(E_{ \pm}\right)$orthogonal to $\operatorname{Ker}\left(D_{ \pm}\right)$and given $\zeta_{\Sigma} \in L_{1}^{2}(\Sigma, \mathbb{C})$, consider

$$
\begin{gathered}
\psi_{-}:=\phi_{-}+\zeta_{-}, \quad \psi_{0}:=\phi_{0}+\mathfrak{n} \zeta_{\Sigma}, \quad \psi_{+}:=\phi_{+}+\zeta_{+}, \\
\psi:=\beta_{-} \psi_{-}+\beta_{0} \psi_{0}+\beta_{+} \psi_{+} \in L_{1}^{2}\left(N_{C}\right) .
\end{gathered}
$$


Recall that the construction of the linear gluing exact sequence (10.23) writes

$$
D_{C}(\psi)=\beta_{-} \Theta_{-}\left(\psi_{-}, \psi_{0}\right)+\beta_{0} \Theta_{0}\left(\psi_{-}, \psi_{0}, \psi_{+}\right)+\beta_{+} \Theta_{+}\left(\psi_{0}, \psi_{+}\right),
$$

where $\Theta_{ \pm}$and $\Theta_{0}$ are defined by analogy with (9.10). Near the ramification points, $\Theta_{0}=D_{C} \psi_{0}$, and so by (10.17) and (10.29), the above equation can be rewritten in the form

$$
D_{C}(\psi)=\beta_{-} \Theta_{-}\left(\psi_{-}, \psi_{0}\right)+\beta_{0} \mathfrak{n}^{0,1} \Theta_{\Sigma}\left(\psi_{-}, \psi_{0}, \psi_{+}\right)+\beta_{+} \Theta_{+}\left(\psi_{0}, \psi_{+}\right),
$$

where $\Theta_{\Sigma} \in L^{2}\left(T^{0,1} \Sigma, \mathbb{C}\right)$.

On the other hand, the derivative of the gluing construction in the direction $\left(r_{-}, r_{+}, X\right)$ defines an alternate expression

$$
D_{C}(\psi)=\beta_{-} \Theta_{-}^{\prime}\left(\psi_{-}, \psi_{0}\right)+\beta_{0} \mathfrak{n}^{0,1} \Theta_{\Sigma}^{\prime}\left(\psi_{-}, \psi_{0}, \psi_{+}\right)+\beta_{+} \Theta_{+}^{\prime}\left(\psi_{0}, \psi_{+}\right)
$$

as follows.

The $\Theta^{\prime}$ 's are first-order differential operators, so to define the expression (10.32) we can assume that $\psi_{ \pm}$and $\psi_{0}$ are smooth. Consider a smooth one-parameter family of triples $\left(R_{-}(\epsilon), R_{+}(\epsilon), \Sigma(\epsilon)\right)$, parameterized by $\epsilon$ in a neighborhood of 0 in $\mathbb{R}$, such that $\left(R_{-}(0), R_{+}(0), \Sigma(0)\right)$ agrees with our given element $\left(R_{-}, R_{+}, \Sigma\right) \in \mathfrak{s}^{-1}(0)$, while $\left.\frac{d}{d \epsilon}\right|_{\epsilon=0} R_{ \pm}(\epsilon)=r_{ \pm}$and $\left.\frac{d}{d \epsilon}\right|_{\epsilon=0} \Sigma(\epsilon)=X$. Let $\widetilde{\zeta}_{ \pm}(\epsilon)$ be a smooth 1-parameter family of sections of the normal bundle to $u_{ \pm}$such that $\widetilde{\zeta}_{ \pm}(0)$ is the section produced by the gluing construction (in Propositions 5.6 and 5.7 and denoted there by $\left.\psi_{ \pm}\right)$applied to $\left(R_{-}, R_{+}, \Sigma\right)$, while $\left.\frac{d}{d \epsilon}\right|_{\epsilon=0} \widetilde{\zeta}_{ \pm}(\epsilon)=\zeta_{ \pm}$.

Fix a small neighborhood $U$ of the ramification points in $\Sigma$. Note that when $\epsilon$ is sufficiently small, there is a canonical diffeomorphism of $\Sigma \backslash U$ with a subset of $\Sigma(\epsilon)$, respecting the projections to $\mathbb{R} \times S^{1}$. Let $\widetilde{\zeta}_{\Sigma}(\epsilon)$ be a 1-parameter family of complex-valued functions on $\Sigma \backslash U$ such that $\widetilde{\zeta}_{\Sigma}(0)$ is the restriction to $\Sigma \backslash U$ of the function on $\Sigma$ produced by the gluing construction (Proposition 5.7) applied to $\left(R_{-}, R_{+}, \Sigma\right)$, and

$$
\left.\frac{d}{d \epsilon}\right|_{\epsilon=0} \widetilde{\zeta}_{\Sigma}(\epsilon)=\mathfrak{n}^{-1} \psi_{0}
$$

To continue, let $C(\epsilon)$ denote the partially defined surface obtained from the pregluing construction applied to $\left(R_{-}(\epsilon), R_{+}(\epsilon), \Sigma(\epsilon)\right)$ using the sections $\left(\widetilde{\zeta}_{-}(\epsilon), \widetilde{\zeta}_{\Sigma}(\epsilon), \widetilde{\zeta}_{+}(\epsilon)\right)$; this is defined over the complement of $U$. Here equation (5.8) writes $\bar{\partial}(C(\epsilon))$ in the form

$$
\begin{aligned}
2 i \bar{\partial}(C(\epsilon))= & \beta_{-}(\epsilon) \widetilde{\Theta}_{-}\left(\widetilde{\zeta}_{-}(\epsilon), \widetilde{\zeta}_{\Sigma(\epsilon)}\right)+\beta_{+}(\epsilon) \widetilde{\Theta}_{+}\left(\widetilde{\zeta}_{\Sigma(\epsilon)}, \widetilde{\zeta}_{+}\right) \\
& +\beta_{0}(\epsilon) \mathfrak{n}^{0,1} \widetilde{\Theta}_{\Sigma(\epsilon)}\left(\widetilde{\zeta}_{-}(\epsilon), \widetilde{\zeta}_{\Sigma(\epsilon)}, \widetilde{\zeta}_{+}(\epsilon)\right)
\end{aligned}
$$

(where $\widetilde{\Theta}$ here corresponds to $\Theta$ in equation (5.8)). Note that on the part of $C$ corresponding to the complement of $U$, the normal derivative of the family of surfaces $\left\{C_{\epsilon}\right\}$ at $\epsilon=0$ is given by the section $\psi$ defined in (10.30). 
Thus differentiating equation (10.34) at $\epsilon=0$, and using the fact that the $\widetilde{\Theta}$ 's vanish at $\epsilon=0$, gives

$$
D_{C}(\psi)=\left.\beta_{-} \frac{\partial}{\partial \epsilon}\right|_{\epsilon=0} \widetilde{\Theta}_{-}+\left.\beta_{0} \mathfrak{n}^{0,1} \frac{\partial}{\partial \epsilon}\right|_{\epsilon=0} \widetilde{\Theta}_{\Sigma(\epsilon)}+\left.\beta_{+} \frac{\partial}{\partial \epsilon}\right|_{\epsilon=0} \widetilde{\Theta}_{+} .
$$

The expansion (10.32) is now defined over the complement of $U$ by setting $\Theta_{ \pm}^{\prime}:=\left.\frac{\partial}{\partial \epsilon}\right|_{\epsilon=0} \widetilde{\Theta}_{ \pm}$and $\Theta_{\Sigma}^{\prime}:=\left.\frac{\partial}{\partial \epsilon}\right|_{\epsilon=0} \widetilde{\Theta}_{\Sigma(\epsilon)}$. Near the ramification points, this expansion agrees with (10.31), and as such has a canonical extension over $U$.

With the preceding understood, for $\tau \in[0,1]$ define

$$
\Theta_{-}^{\tau}:=(1-\tau) \Theta_{-}+\tau \Theta_{-}^{\prime}, \quad \Theta_{\Sigma}^{\tau}:=(1-\tau) \Theta_{\Sigma}+\tau \Theta_{\Sigma}^{\prime}, \quad \Theta_{+}^{\tau}:=(1-\tau) \Theta_{+}+\tau \Theta_{+}^{\prime} .
$$

Keep in mind that the $\Theta^{\tau}$ 's depend implicitly on the triple $\left(r_{-}, r_{+}, X\right) \in$ $\mathbb{R}^{2} \oplus T_{\Sigma} \mathcal{M}$. Note that one can write

$$
\begin{gathered}
\Theta_{-}^{\tau}=D_{-} \zeta_{-}+\mathcal{R}_{-}^{\tau}\left(\zeta_{-}, \zeta_{\Sigma}\right), \quad \Theta_{+}^{\tau}=D_{+} \zeta_{+}+\mathcal{R}_{+}^{\tau}\left(\zeta_{\Sigma}, \zeta_{+}\right), \\
\Theta_{\Sigma}^{\tau}=D_{\Sigma} \zeta_{\Sigma}+\left(\mathfrak{n}^{0,1}\right)^{-1} D_{0} \phi_{0}+\mathcal{R}_{\Sigma}^{\tau}\left(\zeta_{-}, \zeta_{\Sigma}, \zeta_{+}\right),
\end{gathered}
$$

where each term in $\mathcal{R}_{ \pm}^{\tau}$ or $\mathcal{R}_{\Sigma}^{\tau}$ that is linear in $\left(\zeta_{-}, \zeta_{\Sigma}, \zeta_{+}\right)$maps from $L_{1}^{2}$ to $L^{2}$ with small operator norm when $r$ is large. It follows by the usual arguments that if $r$ is sufficiently large, then there exist unique $\zeta_{ \pm} \in L_{1}^{2}\left(E_{ \pm}\right)$that are $L^{2}$-orthogonal to $\operatorname{Ker}\left(D_{ \pm}\right)$, and $\zeta_{\Sigma} \in L_{1}^{2}(\Sigma, \mathbb{C})$, such that

$$
\Theta_{-}^{\tau}\left(\psi_{-}, \psi_{0}\right)=0, \quad \Theta_{\Sigma}^{\tau}\left(\psi_{-}, \psi_{0}, \psi_{+}\right) \in \operatorname{Coker}\left(D_{\Sigma}\right), \quad \Theta_{+}^{\tau}\left(\psi_{0}, \psi_{+}\right)=0 .
$$

Moreover, $\zeta_{ \pm}$and $\zeta_{\Sigma}$ vary continuously with $\tau$. For this distinguished $\zeta_{ \pm}$ and $\zeta_{\Sigma}$, we define

$$
g_{\tau}\left(r_{-}, r_{+}, X\right):=\Theta_{\Sigma}^{\tau}\left(\psi_{-}, \psi_{0}, \psi_{+}\right) .
$$

To complete the construction of the exact sequence (10.26), note that by (10.31) and (10.32), we have a map

$$
\begin{aligned}
\operatorname{Ker}\left(g_{\tau}\right) & \longrightarrow \operatorname{Ker}\left(D_{C}\right), \\
\left(r_{-}, r_{+}, X\right) & \longmapsto \beta_{-} \psi_{-}+\beta_{0} \psi_{0}+\beta_{+} \psi_{+} .
\end{aligned}
$$

A linear version of Lemmas 7.4 and 7.5 shows that if $r$ is sufficiently large, then the map (10.36) is an isomorphism. We now define $f_{\tau}$ to be the inverse of the map (10.36). Thus $f_{\tau}$ is injective and $\operatorname{Im}\left(f_{\tau}\right)=\operatorname{Ker}\left(g_{\tau}\right)$. Since $C$ is unobstructed, $\operatorname{dim} \operatorname{Ker}\left(D_{C}\right)=2$, and so by dimension counting, the sequence (10.26) is exact.

We now show that when $\tau=1$, the exact sequence (10.26) agrees with (10.2). We first show that $g_{1}=\nabla \mathfrak{s}$. Let $\left(r_{-}, r_{+}, X\right) \in \mathbb{R}^{2} \oplus T_{\Sigma} \mathcal{M}$ be given, and let $\phi_{ \pm}, \phi_{0}$, and $\left(R_{-}(\epsilon), R_{+}(\epsilon), \Sigma(\epsilon)\right)$ be as before. For each $\epsilon$, the gluing construction finds a unique triple $\left(\widetilde{\zeta}_{-}(\epsilon), \widetilde{\zeta}_{\Sigma}(\epsilon), \widetilde{\zeta}_{+}(\epsilon)\right)$, where $\widetilde{\zeta}_{ \pm}(\epsilon)$ is an $L_{1}^{2}$ section of $u_{ \pm}$orthogonal to $\operatorname{Ker}\left(D_{ \pm}\right)$, and $\widetilde{\zeta}_{\Sigma}(\epsilon) \in L_{1}^{2}(\Sigma(\epsilon), \mathbb{C})$, such 
that $\widetilde{\Theta}_{ \pm}=0$ and $\widetilde{\Theta}_{\Sigma(\epsilon)} \in \operatorname{Coker}\left(D_{\Sigma(\epsilon)}\right)$. These depend smoothly on $\epsilon$ (see Section 6.3 ), and by definition,

$$
\nabla \mathfrak{s}\left(r_{-}, r_{+}, X\right)=\left.\frac{d}{d \epsilon}\right|_{\epsilon=0} \widetilde{\Theta}_{\Sigma(\epsilon)} \in \operatorname{Coker}\left(D_{\Sigma}\right) .
$$

Now define $\zeta_{ \pm}:=\left.\frac{d}{d \epsilon}\right|_{\epsilon=0} \widetilde{\zeta}_{ \pm}(\epsilon) \in L_{1}^{2}\left(E_{ \pm}\right)$and $\psi_{ \pm}:=\phi_{ \pm}+\zeta_{ \pm}$. Also, define $\psi_{0} \in L_{1}^{2}\left(E_{0}\right)$ as follows. Off of the ramification points, $\psi_{0}$ is given by equation (10.33). In a neighborhood of the ramification points, $\psi_{0}$ is the normal derivative of the family of surfaces $C(\epsilon)$. Note that $\rho\left(\psi_{0}\right)=X$. Moreover, near the ramification points, $D_{C} \psi_{0}=\mathfrak{n}^{0,1} \nabla \mathfrak{s}$. It follows as in the proof of Lemma 10.6 that $\psi_{0}-\phi_{0}=\mathfrak{n} \zeta_{\Sigma}$ for some $\zeta_{\Sigma} \in L_{1}^{2}(\Sigma, \mathbb{C})$. The triple $\left(\zeta_{-}, \zeta_{\Sigma}, \zeta_{+}\right)$is then the unique solution to equation $(10.35)$, so by the definition of $g_{1}$ and equation (10.37) we conclude that $g_{1}\left(r_{-}, r_{+}, X\right)=$ $\nabla \mathfrak{s}\left(r_{-}, r_{+}, X\right)$. Similarly, $f_{1}=\mathcal{I}$.

Part 2. We now define the map $\widetilde{\rho}$ in (10.27). Let $\left(\phi_{-}, \phi_{0}^{\prime}, \phi_{+}\right)$be given, where $\phi_{ \pm} \in \operatorname{Ker}\left(D_{ \pm}\right)$and $\phi_{0}^{\prime} \in \operatorname{Ker}\left(\Pi_{W_{0}} D_{0}\right)$. As in the definition of the map $g$ in $(10.23)$, there are unique $\zeta_{ \pm} \in L_{1}^{2}\left(E_{ \pm}\right)$orthogonal to $\operatorname{Ker}\left(D_{ \pm}\right)$ and $\zeta_{0} \in L_{1}^{2}\left(E_{0}\right)$ orthogonal to $\operatorname{Ker}\left(\Pi_{W_{0}} D_{0}\right)$ such that $\psi_{ \pm}:=\phi_{ \pm}+\zeta_{ \pm}$and $\psi_{0}:=\phi_{0}^{\prime}+\zeta_{0}$ satisfy

$$
\Theta_{-}\left(\psi_{-}, \psi_{0}\right)=0, \quad \Theta_{+}\left(\psi_{0}, \psi_{+}\right)=0, \quad \Theta_{0}\left(\psi_{-}, \psi_{0}, \psi_{+}\right) \in V_{0} .
$$

Near the ramification points, $\Theta_{0}=D_{0} \psi_{0}$, so as in the proof of Lemma 10.6, $\rho\left(\psi_{0}\right)$ is defined. Let $r_{ \pm}$correspond to $\phi_{ \pm}$under our usual identification $\operatorname{Ker}\left(D_{ \pm}\right) \simeq \mathbb{R}$, and define

$$
\widetilde{\rho}\left(\phi_{-}, \phi_{0}^{\prime}, \phi_{+}\right):=\left(r_{-}, r_{+}, \rho\left(\psi_{0}\right)\right) .
$$

We now show that the diagram (10.28) commutes. To see that the right square commutes, continue with the notation from the definition of $\widetilde{\rho}$, and let $\phi_{0}$ denote the unique element of $\operatorname{Ker}\left(\Pi_{W_{0}} D_{0}\right)$ for which $\rho\left(\phi_{0}\right)=\rho\left(\psi_{0}\right)$. As in the proof of Lemma 10.6,

$$
\psi_{0}=\phi_{0}+\mathfrak{n} \zeta_{\Sigma}
$$

for some $\zeta_{\Sigma} \in L_{1}^{2}(\Sigma, \mathbb{C})$. Then $\left(\psi_{-}, \psi_{0}, \psi_{+}\right)$is the unique solution to equation (10.35) for $\tau=0$ and $\left(r_{-}, r_{+}, X\right)=\widetilde{\rho}\left(\phi_{-}, \phi_{0}^{\prime}, \phi_{+}\right)$. So by definition,

$$
\begin{aligned}
\mathfrak{n}^{0,1} g_{0} \widetilde{\rho}\left(\phi_{-}, \phi_{0}^{\prime}, \phi_{+}\right) & =\mathfrak{n}^{0,1} \Theta_{\Sigma}\left(\psi_{-}, \psi_{0}, \psi_{+}\right) \\
& =\Theta_{0}\left(\psi_{-}, \psi_{0}, \psi_{+}\right) \\
& =g\left(\phi_{-}, \phi_{0}^{\prime}, \phi_{+}\right) .
\end{aligned}
$$

Similarly, the left square in (10.28) commutes.

Part 3. We now show that $\widetilde{\rho}$ is an isomorphism, which is homotopic through isomorphisms to the map sending $\left(\phi_{-}, \phi_{0}^{\prime}, \phi_{+}\right) \mapsto\left(r_{-}, r_{+}, \rho\left(\phi_{0}^{\prime}\right)\right)$. To see this, for $\tau \in[0,1]$ consider the linear interpolation

$$
\widetilde{\rho}_{\tau}\left(\phi_{-}, \phi_{0}^{\prime}, \phi_{+}\right):=\left(r_{-}, r_{+}, \tau \rho\left(\psi_{0}\right)+(1-\tau) \rho\left(\phi_{0}^{\prime}\right)\right) .
$$


It is enough to show that $\widetilde{\rho}_{\tau}$ is injective for each $\tau$. Suppose on the contrary that $\widetilde{\rho}_{\tau}\left(\phi_{-}, \phi_{0}^{\prime}, \phi_{+}\right)=0$. Then by Lemma 10.6 ,

$$
\begin{aligned}
\phi_{ \pm} & =0 \\
\tau \phi_{0}+(1-\tau) \phi_{0}^{\prime} & =0 .
\end{aligned}
$$

It follows from (10.39), as in the definition of $g$ in Proposition 9.3, that for any $\varepsilon>0$, if $r, T_{ \pm}$are large, then

$$
\begin{aligned}
\left\|\psi_{ \pm}\right\|_{L_{1}^{2}} & \leq \varepsilon\left\|\phi_{0}^{\prime}\right\|_{L_{1}^{2}}, \\
\left\|\psi_{0}-\phi_{0}^{\prime}\right\|_{L_{1}^{2}} & \leq \varepsilon\left\|\phi_{0}^{\prime}\right\|_{L_{1}^{2}} .
\end{aligned}
$$

The inequality (10.41), together with the equation $\Theta_{0}\left(\psi_{-}, \psi_{0}, \psi_{+}\right) \in V_{0}$, implies as in the proof of Lemma 10.6 that the function $\zeta_{\Sigma}$ defined in (10.38) satisfies

$$
\left\|\zeta_{\Sigma}\right\|_{L_{1}^{2}} \leq \varepsilon\left\|\phi_{0}^{\prime}\right\|_{L_{1}^{2}}
$$

if $r$ is sufficiently large. On the other hand, equations (10.38) and (10.40) imply that

$$
\phi_{0}^{\prime}=\tau\left(\left(\phi_{0}^{\prime}-\psi_{0}\right)+\mathfrak{n} \zeta_{\Sigma}\right) .
$$

This contradicts (10.42) and (10.43) if $\varepsilon$ is chosen sufficiently small.

This completes the proof of Lemma 10.7, and thus Theorem 10.2 is proved.

\section{References}

[1] F. Bourgeois, Y. Eliashberg, H. Hofer, K. Wysocki and E. Zehnder, Compactness results in symplectic field theory, Geom. Topol. 7 (2003), 799-888.

[2] F. Bourgeois and K. Mohnke, Coherent orientations in symplectic field theory, Math. Z. 248 (2004), 123-146.

[3] K. Cieliebak and K. Mohnke, Compactness for punctured holomorphic curves, J. Symplectic Geom. 3 (2005), 589-654.

[4] S.K. Donaldson and P.B. Kronheimer, The geometry of four-manifolds, Oxford University Press, 1990.

[5] D. Dragnev, Fredholm theory and transversality for noncompact pseudoholomorphic maps in symplectizations, Comm. Pure Appl. Math 57 (2004), 726-763.

[6] A. Floer and H. Hofer, Coherent orientations for periodic orbit problems in symplectic geometry, Math. Z. 212 (1993), 13-38.

[7] H. Hofer, K. Wysocki and E. Zehnder, Properties of pseudoholomorphic curves in symplectizations. I. Asymptotics, Ann. Inst. H. Poincare Anal. Non Lineaire 13 (1996), 337-379.

[8] M. Hutchings and M. Sullivan, Rounding corners of polygons and the embedded contact homology of $T^{3}$, Geom. Topol. 10 (2006), 169-266.

[9] M. Hutchings and C.H. Taubes, Gluing pseudoholomorphic curves along branched covered cylinders I, J. Symplectic Geom. 5 (2007), 43-137. 
[10] D. McDuff and D. Salamon, J-holomorphic curves and symplectic topology, AMS, 2004.

[11] E. Mora, Pseudoholomorphic cylinders in symplectizations, NYU PhD thesis, 2003.

[12] C.B. Morrey, Multiple integrals in the calculus of variations, Springer-Verlag, 1966.

[13] R. Siefring, The relative asymptotic behavior of pseudoholomorphic half-cylinders, to appear in Comm. Pure Appl. Math., arXiV:math/0702356.

[14] C.H. Taubes, $S W \Rightarrow$ Gr: from the Seiberg-Witten equations to pseudo-holomorphic curves, 'Seiberg Witten and Gromov Invariants for Symplectic Manifolds', International Press, 2000.

[15] C. Wendl, Punctured holomorphic curves with boundary in 3-manifolds: Fredholm theory and embeddedness, in preparation.

Department of Mathematics

970 Evans Hall

UNIVERSITY OF CALIFORNIA

BERKELEY

CA 94720

E-mail address: hutching@math.berkeley.edu

Department of Mathematics

HARVARD UNIVERSITY

1 OXFord STREET

CAMBRIDGE

MA 02138-2901

E-mail address: chtaubes@math.harvard.edu

Received 5/16/2007, accepted 12/19/2008

M.H. was partially supported by NSF grant DMS-0505884 and the Alfred P. Sloan Foundation. C.T. was partially supported by the National Science Foundation. 
